\title{
Future health in a globalising world
}

Citation for published version (APA):

Huynen, M. M. T. E. (2008). Future health in a globalising world. [Doctoral Thesis, Maastricht University]. Universitaire Pers Maastricht. https://doi.org/10.26481/dis.20080411mh

Document status and date:

Published: 01/01/2008

DOI:

10.26481/dis.20080411mh

Document Version:

Publisher's PDF, also known as Version of record

\section{Please check the document version of this publication:}

- A submitted manuscript is the version of the article upon submission and before peer-review. There can be important differences between the submitted version and the official published version of record.

People interested in the research are advised to contact the author for the final version of the publication, or visit the DOI to the publisher's website.

- The final author version and the galley proof are versions of the publication after peer review.

- The final published version features the final layout of the paper including the volume, issue and page numbers.

Link to publication

\footnotetext{
General rights rights.

- You may freely distribute the URL identifying the publication in the public portal. please follow below link for the End User Agreement:

www.umlib.nl/taverne-license

Take down policy

If you believe that this document breaches copyright please contact us at:

repository@maastrichtuniversity.nl

providing details and we will investigate your claim.
}

Copyright and moral rights for the publications made accessible in the public portal are retained by the authors and/or other copyright owners and it is a condition of accessing publications that users recognise and abide by the legal requirements associated with these

- Users may download and print one copy of any publication from the public portal for the purpose of private study or research.

- You may not further distribute the material or use it for any profit-making activity or commercial gain

If the publication is distributed under the terms of Article $25 \mathrm{fa}$ of the Dutch Copyright Act, indicated by the "Taverne" license above, 
Future health in a globalising world

Maud Huynen 
Cover graphic: $($ Media for Development International, Courtesy of Photoshare Copyright: $(2008$, Maud Huynen

ISBN 9789052787084 


\section{Future health in a globalising world}

\author{
PROEFSCHRIFT \\ ter verkrijging van de graad van \\ doctor aan de Universiteit \\ Maastricht, \\ op gezag van de Rector Magnificus, \\ Prof. mr. G.P.M.F. Mols, \\ volgens het besluit van het College \\ van Decanen, \\ in het openbaar te verdedigen \\ op
}

vrijdag 11 april 2008 om 14:00 uur

door

\author{
DISSERTATION \\ to obtain the degree of Doctor at the \\ Maastricht University, \\ on the authority of the Rector \\ Magnificus, \\ Prof. dr. G.P.M.F. Mols, \\ in accordance with the decision of \\ the Board of Deans, \\ to be defended in public \\ on \\ Friday April 11 th 2008 at 14:00 hours \\ by
}

Maud Maria Theresia Elisabeth Huynen

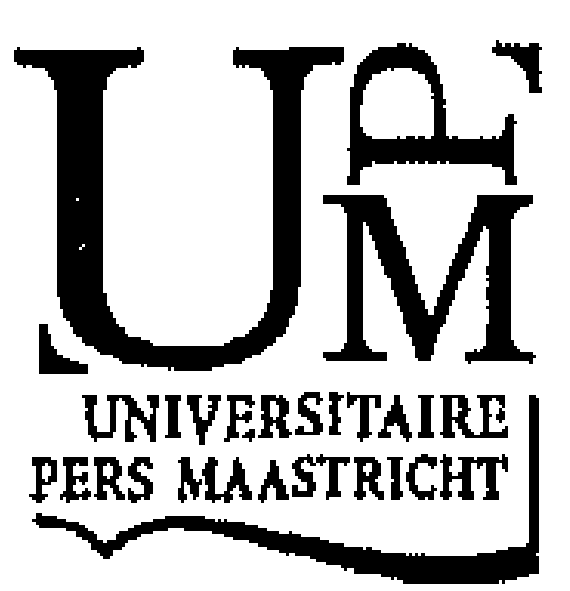




\section{Promotor}

Prof. dr. Pim Martens

\section{Copromotor}

Dr. Henk B.M. Hilderink

Netherlands Environmental Assessment Agency (MNP), the Netherlands

\section{Beoordelingscommissie/Assessment Committee}

Prof. dr. Wim F. Passchier (chair)

Dr. Rene Kemp

Prof. dr. Johan P. Mackenbach, Erasmus MC, University Medical Centre Rotterdam, the Netherlands

Prof. dr. Colin L. Soskolne, Department of Public Health Sciences,

School of Public Health, University of Alberta, Canada

This Ph.D. research was supported by the Netherlands Environmental Assessment Agency (MNP). 
Prediction is very difficult, especially about the future.

Niels Bohr (1885 - 1962)

The trouble with our times is that the future is not what it used to be.

Paul Valery $(1871$ - 1945)

Enjoy present pleasures in such a way as not to injure future ones.

Seneca $(5 \mathrm{BC}-65 \mathrm{AD})$

The function of science fiction is not always to predict the future but sometimes to prevent it.

Frank Herbert (1920 - 1986)

The future belongs to those who prepare for it today.

Malcolm X (1925 - 1965) 


\section{CONTENTS}

1 Introduction - 1

1.1 The importance of exploring future health: a sustainability perspective -5

1.2 Definition of main concepts --.- 6

1.2.1 Population health --

1.2.2 Globalisation - 8

1.3 Scope and objectives-11

1.4 Outline--

2 Methodological framework -

2.1 Globalisation and population health: the complexities involved----o----17

2.2 Integrated Assessment--

2.2.1 Integrated Assessment models -

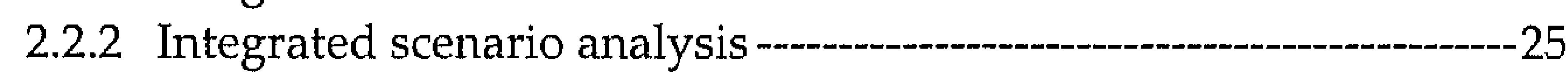

2.2.3 Participatory methods -_-

2.3 Research approach--.--

3 Past developments in our health: the epidemiological transition --.----31

3.1 The epidemiological transition framework -

3.1.1 The classical or western transition model -._-

3.1.2 The non-western transition model --_-37

3.2 The epidemiological transition in the Netherlands --_-_--39

3.2.1 Health developments in the Netherlands: the western model -----40

3.2.2 The fourth transition stage in the Netherlands ---

3.2.3 Discussion -.-_-_-_-_-_---56

3.3 The epidemiological transition in Peru

3.3.1 Health developments in Peru: the non-western model ---_-_-----59

3.3.2 Epidemiological polarisation in Peru -

3.3.3 Discussion - -

3.4 The epidemiological transition from a systems perspective ---_-_-_-_--69

3.4.1 The drivers of epidemiological change -.--70

3.4.2 Critiques on the epidemiological transition concept -..-_-..--.--.--72

3.4.3 Lessons learned-_-_-_- 
4 Globalisation and health: a conceptual model-a-n

4.1 Review of existing health models

4.2 A new framework for population health -

4.3 Features of globalisation --.- 92

4.4 Conceptual framework for globalisation and health -._-_._-_._-_._-98

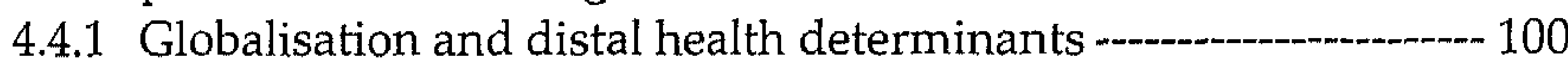

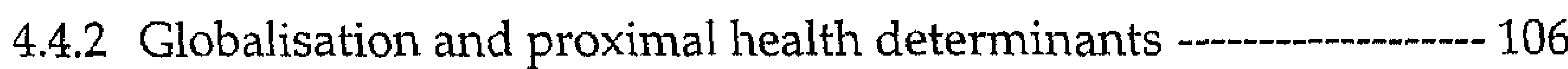

4.4.3 Discussion of conceptual framework- 112

5 A future without health? --_- 113

5.1 Selected scenario studies --..-...- 115

5.2 The health dimension in global scenario studies --_- 122

5.3 Linking scenarios to health --.- 125

5.3.1 Possible health futures

5.3.2 Future health in the SRES-scenarios and the GEO3-scenarios --- 128

5.3.3 Future health in the MA-scenarios-_- 133

5.3.4 Future health in a globalising world --_-_- 145

5.4 Discussion -...- 147

6 Climate change, temperature and mortality in the Netherlands ---a---- 151

6.1 Climate change, temperature and mortality

6.2 Temperature and mortality in the Netherlands-_- 156

6.2.1 Methods - 157

6.2.2 The mortality-temperature relationship in the Netherlands----- 163

6.2.3 Excess mortality during heat waves and cold spells --.-.-.-.-- 166

6.2.4 Discussion and conclusion--.-1 171

6.3 Climate change, temperature change and mortality--_-_-_- 174

6.3.1 Methods -.-_- 175

6.3.2 Temperature-related mortality attributable to climate change--- 180

6.3.3 Discussion and conclusion--- 185

Appendix to Chapter 6 -..- 189

7 Linkages between biodiversity loss and health -._- 193

7.1 Biodiversity, ecosystem functioning and human health

7.2 Methods - 199

7.2.1 Selected indicators

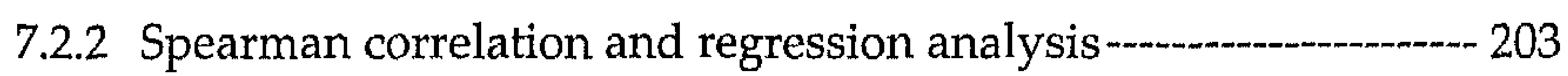

7.3 Results -..-_- 204

7.3.1 Crude associations between health and biodiversity-_-_-_-_-_- 205

7.3.2 Adjusted associations between health and biodiversity --------- 206

7.4 Discussion and conclusions -_._- 210 


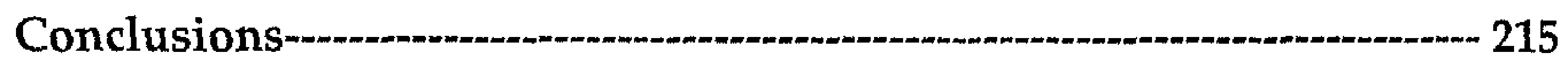

8.1 Past developments in our health: the epidemiological transition ------ 218

8.2 Globalisation and health: a conceptual framework --- 219

8.3 Future health in a globalising world -.-- 220

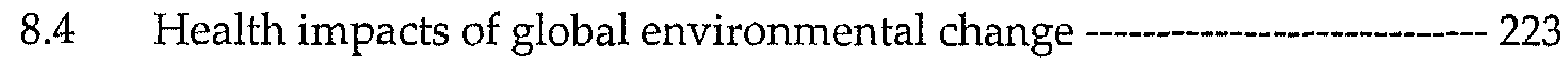

8.4.1 Climate change and temperature-related mortality in the

Netherlands - 223

8.4.2 Global loss of biodiversity and health - 225

8.5 Methodological reflection -- 226

8.5.1 Holism and systems thinking -.-_- 227

8.5.2 Uncertainty and plurality -.- 228

8.5.3 Inter- and transdisciplinarity-- 229

8.6 Globalisation and health: future outlook

8.6.1 Developing global health scenarios: the road ahead --.--------- 230

8.6.2 Assessing the health impacts of global environmental change -- 232

8.6.3 Global health: a priority for the international agenda--_-_-_--233

8.6.4 Epilogue-_-_- 234

References 237

Summary - 261

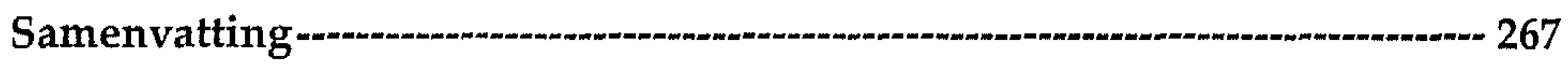

Dankwoord \& Acknowledgements -..... 273

Curriculum vitae --1.-- 277 
Chapter 1

INTRODUCTION 


\section{INTRODUCTION}

Today it is acknowledged that achieving sustainable development at the global scale is one of the greatest challenges for the 21st century. In light of this international commitment to sustainable development, exploring our future has become a matter of urgency and relevance. How will globalisation evolve? Are we on a sustainable pathway? Will we be able to sustain ourselves as well as our planet? Obviously, studying the future of our health should be a vital part of this; the intergenerational equity implied by sustainable development forces us to think about the right of future generations to a healthy life (Huynen, 2003).

Looking at the health of human populations, it is evident that we are living through what is, in historical terms, a spectacular heath transition: life expectancy significantly increased and mortality fell sharply, as did birth rates. Still, health inequalities between rich and poor continue to persist and good health for all populations has become an accepted international goal (see, for example, the Millennium Development Goals (MDG)). In our attempts to realise this goal, however, we have to recognise that the prospects for future health more and more depend on the relative new processes of globalisation.

In the past, globalisation has often been seen as a more or less economic process characterised by increased deregulated trade, electronic communication, and capital mobility. However, globalisation is becoming increasingly perceived as a more comprehensive phenomenon that is shaped by a multitude of factors and events, and which is reshaping our society rapidly; it encompasses not only economic, political, and technological forces, but also social-cultural and environmental aspects. Globalisation is an overarching process in which simultaneously many different processes take place in many different domains (e.g., governance structures, markets, communication, mobility, cultural interactions, and environmental change). As a result, the geographical scale of important health issues is significantly increasing. In 2003, for example, the world held its breath during a historically unprecedented situation: the rapid spread of the Severe Acute Respiratory Syndrome (SARS) caused by worldwide human travelling (Box 1.1). As Dr. Margaret Chan, the current Director-General of the World Health Organization (WHO), stated: 'SARS was an excellent example in demonstrating to countries that because infectious diseases do not respect borders (...) an outbreak in one country one day can very rapidly become a problem for countries on the other side of the world (...)' (WHO, 2006). Fortunately, the global actions taken by WHO played a decisive role in fighting this disease. In the aftermath, we asked ourselves the question whether this was a sign of things to come. 
Box 1.1: The rapid spread of SARS in 2003 (WHO, 2003a, 2003b).

The first cases of the Severe Acute Respiratory Syndrome (SARS) probably arose in November 2002 in Foshan City, Guangdong Province of China. In February 2003, the Chinese Ministry of Health reported 305 cases. SARS was first reported outside of China in on March 28th 2003, when WHO official Dr. Carlo Urbani reported several cases of 'atypical pneumonia' at the Vietnamese hospital where he worked. The first of these cases was a Chinese-American businessman. Before going to Vietnam, the businessman had stayed on the ninth floor of the Metropole Hotel in Hong Kong. At the same time, a doctor from Guangdong Province had been a guest on the same floor. This doctor had treated SARS patients in Guangdong prior to his visit to Hong Kong, thus carrying the disease out of China.

It soon became clear that the Guangdong doctor had infected 12 other guests and visitors of the Metropole hotel. Among them were a tourist from Toronto, a young woman from Singapore, the businessman who subsequently travelled to Vietnam, and a local Hong Kong resident who was visiting an acquaintance at the hotel. Because it can take up to 10 days for a person who is infected with SARS to actually get sick, guests of the hotel unwittingly carried the disease out of Hong Kong via air travel into Canada, Vietnam, and Singapore. When these patients did get sick, they were not diagnosed right away; patients' families and health care providers were exposed and became infected with the disease as well.

Within 3 weeks after Dr. Urbani's report, cases had been reported from around the all world, including Canada, Germany, Taiwan (China), Thailand, the United Kingdom, The United States, Hong Kong, Viet Nam, and Singapore.

The link between global mobility and the spread of infectious diseases is perhaps the best-known health effect of globalisation. However, it is only one of the many possible health implications of globalisation. A variety of both negative and positive effects are expected to influence our health in the (near) future (see Chapter 4), but it is still very uncertain which are going to prevail and current literature shows an ongoing polarised debate (Lee, 2001). Baum (2001), for example, states that 'the current forms of globalisation are making the world a safe place for unfettered market liberalism and the consequent growth of inequities. This economic globalisation is posing severe threats to both people's health and the health of the planet'. On the other hand, Feachem (2001), for example, argues that the risks and adverse consequences of globalisation must be confronted, but they must not be allowed to obscure its overall positive impact on health and development'. Hence, optimists argue that globalisation will be beneficial for population health, while others are increasingly concerned about the negative consequences. Due to prevailing uncertainties, all the opposing interpretations and perspectives have to be considered legitimate. Therefore, it is hard for both scientists and policymakers to discuss how global development should move forward in this respect (Lee, 2001). Still, this deadlock has to be tackled.

The next section, first discusses- from a sustainability perspective- the importance of exploring our future health. Accordingly, the main concepts within this Ph.D. Thesis, namely population health and globalisation, are 
defined. Finally, the main research question and objectives, as well as the outline of this Ph.D. Thesis are presented.

\subsection{THE IMPORTANCE OF EXPLORING FUTURE HEALTH: A SUSTAINABILITY PERSPECTIVE}

In the past decade, promoting good health has been recognised as one of the key elements in achieving a sustainable form of globalisation (Lee, 2003) and our (future) health should be an integral part of the current discussions about sustainable development and sustainable globalisation. One of the first definitions of sustainable development was published in the 1987 report 'Our common future' by the Brundtland Commission (Brundtland, 1987): 'development that meets the needs of the present without compromising the ability of future generations to meet their own needs'. The report highlighted three fundamental components to sustainable development: environmental protection, economic growth and social equity. Moreover, a fundamental element in this report was that sustainability cannot be achieved if poverty is not reduced worldwide.

Many other definitions of sustainable development and interpretations have been developed since. Grosskurth and Rotmans (2004) argue that one important denominator of these definitions is an implied balance of economic, ecological and social developments. However, when it comes to the analytical and theoretical underpinnings, the social dimension has been commonly recognised as the weakest 'pillar' of sustainable development (Lehtonen, 2004). Two other denominators that can be identified are the achievement of human well-being for all (intergenerational equity), and the balance between current and future generations (intergenerational equity).

However, it is important to note that the term 'sustainable development' has been criticised for implying further economic growth rather than achieving a sustainable path into the future. Bensimon and Benatar (2006), for example argue that development should not be considered in economic terms, but should reflect other important aspects that are needed for a decent human life as well (e.g. education, a healthy living environment and democracy). As such, they propose to talk in terms of 'the development of sustainability' rather than 'sustainable development', as the latter does not move beyond the narrow conception of development as an economic process.

The Brundtland report argues that the satisfaction of human needs and aspirations is the major objective of development' and 'sustainable development requires meeting the basic needs of all and extending to all the opportunity to satisfy their aspirations for a better life'. There have been several attempts to identify what our basic needs actually encompass. Well-known theories are the ones developed by Maslow $(1954,1968)$ and Max-Neef (1991), 
and in both approaches maintaining or improving our physical (and mental) health is seen as a crucial element. This awareness that health is a fundamental part in any approach towards human (basic) needs, and, therefore, central to sustainable development also shows from the following quote by Bradley et al. (2001): 'If sustainable development is to mean anything, people must be healthy enough to benefit from it and not have their lives cut off prematurely. Development without health is meaningless'.

The relationship between sustainable development and population health works two ways. As the world around us is becoming progressively interconnected and complex, human health is increasingly perceived as the integrated outcome of its ecological, social-cultural, economic and institutional determinants. Due to its multidimensional causality (see also Chapter 4), good health is often seen as an outcome of sustainable development. McMichael $(2000,2006)$ argues that health can be seen as an important high-level integrating index that reflects the state -and, in the long term, the sustainabilityof our natural and socio-economic environment. The increasing widespread and long-term risks to population health are, therefore, at the heart of nonsustainability. Wilcox and Colwell (2005), for example, agree that no issue could be a more fundamental measure of sustainability than public health. The other way around, however, a healthy population is also necessary to achieve sustainable development. As Brundtland (2002), Director-General Emeritus of the WHO, puts it: 'a healthy life is an outcome of sustainable development, as well as a powerful and undervalued means of achieving it.' The WHO Commission on Macroeconomics and Health (WHO, 2001a), for example, concluded that good health is a central input to poverty reduction and socioeconomic development.

\subsection{DEFINITION OF MAIN CONCEPTS}

The definition of the main concepts should be the starting point of any scientific study, especially if these definitions are the topics of debate themselves. Therefore, a clear definition of both population health and globalisation is needed to ensure internal coherence and to develop sound descriptions, explanations and argumentations within this Ph.D. Thesis.

\subsubsection{Population health}

Good health for all populations has become an accepted international goal, but good health means different things to different people, and its meaning varies according to individual and community expectations and context. This subjectivity makes it very difficult to define (good) health. Table 1.1 gives 
several examples of existing definitions of health, divided into three groups: 1) definitions describing health as a state, 2) definitions describing health as a resource or capacity and 3) definitions describing health as an outcome (Huynen \& Martens, 2002).

Table 1.1: Different conceptions and definitions of health (Huynen \& Martens, 2002).

\section{Health as a state}

Health is a state of complete physical, mental and social well-being, and not merely the absence of disease or infirmity (WHO, 1946).

Health is the condition of being sound in body, mind or general spirit, especially freedom from physical disease or pain (Ninth New Collegiate Webster's Dictionary, 1977).

Optimal health is a balance of physical, emotional, social and spiritual well-being (O'Donnell, 1989).

Health is the absence of diseases and disability (Larson, 1999).

A state of equilibrium between humans and the physical, biological, and social environment, compatible with full functional activity (Last, 1997).

\section{Health as a resource/capacity.}

Health is a positive concept emphasising social and personal resources, as well as physical capacities (WHO, 1986).

Health is an integrated method of function, which is oriented toward maximising the potential of which the individual is capable of within the environment where he is functioning (Dunn, 1977). Health is the capacity of people to adapt to, respond to, or control life's challenges and changes (Frankish et al., 1996).

\section{Healthas an outcome}

Health is an outcome of family functional and social support, resourcefulness and versatility (Kane, 1988).

Population health is determined by a complex mixture of genetic, environmental and social factors, as well as individual behaviour (Scally, 1998).

A distinction can be made between individual and population health. Traditional medical thinking has been largely concerned with individuals that were already sick or those that were at the greatest risk of developing a health problem. In order to understand and improve the health status of an entire population a broader understanding of health and its interrelated institutional, economic, social-cultural, and ecological determinants is required (IOM, 2003). As this Ph.D. Thesis aims to assess the possible health impacts of the globalisation process, we prefer to regard health as an outcome. Hence, population health is defined as the integrated outcome of the institutional, economic, social-cultural, and ecological determinants that affect a population's physical, mental and social abilities to function normally. Our focus will primarily be on the physical aspects of population health. Chapter 4 provides a detailed overview of the multiple determinants of population health. 
Population health can refer to health at different geographical scales ranging from a (small) community (community health) to an entire country (national health) or beyond (international health). In addition, our health is also becoming more and more affected by factors that transcend national borders (transnational health) and the implications of globalisation are leading to new patterns of health and disease that do not necessarily conform to, or are revealed by, national boundaries alone. Lee (2002) argues that one speaks of such transnational or transborder health issues, when the causes or consequences of a health issue circumvent, undermine or are oblivious to the territorial boundaries of states and, thus, beyond the capacity of the states to address effectively through state institutions alone'. Thus, transborder health issues are not confined to a specific country or group of countries, although great inequities in impact are being experienced within and across populations. According to Lee (2002) it is, however, often difficult to make the distinction between international and transnational health in practice as 'many of today's health issues are, in theory, international health issues, but in reality governments often do not have the capacity or will to deal with them properly'.

\subsubsection{Globalisation}

Globalisation is not an abstract concept; it does not refer to a concrete object, but to an interpretation of a societal process (Lubbers \& Koorevaar, 1999). The term globalisation first appeared in a dictionary in the early 1960s, but only came into widespread use in the 1980s. Which brings us to the question why globalisation is such a hot issue now, but not 30 years ago? Are we merely dealing with the rise of a buzzword or is something else going on (Scholte, 2000)?

In their analysis of globalisation, Held et al. (2000) describe a widening, deepening, and speeding up of global interconnectedness, resulting in an acceleration of interdependence, action-at-distance, and time-space compression. They define globalisation as 'a process (or set of processes) which embodies a transformation in the spatial organisation of social relations and transactions- assessed in terms of their extensity, intensity, velocity and impactgenerating transcontinental or interregional flows and networks of activity, interaction, and the exercise of power'. Extensity of global networks refers to the widening 'reach' of networks and stretching-up activities across frontiers. Intensity of global interconnectedness refers to the growing magnitude of interconnectedness, the connections across frontiers are not occasional, but they are regularised. The velocity of global flows and interchanges refers to the speeding up of global interactions and processes (e.g., transport, and communication technology). 
Held et al. (1999) distinguish three stereotypical views in historical analyses of globalisation: a sceptical approach, a hyperglobalist approach and the transformationalist thesis. Those who follow the sceptical line argue that internationalisation and global connections are by no means new phenomena. The globalisation sceptics argue that the extent of 'globalisation' is wholly exaggerated; as international interdependence has existed for centuries, the historical evidence at best confirms only heightened levels of internationalisation. The hyperglobalist approach, on the other hand, does not deny the importance of previous developments of growing interdependence, but identifies globalisation as a new epoch of human history characterised by 'denationalisation' and resulting in a global age. The followers of the transformationalist thesis argue that globalisation is not a new phenomenon, but a long-term historical process; however, current levels of global interconnectedness are historically unprecedented and contemporary globalisation is perceived as a dynamic and open-ended process, which is transforming modern societies and the world order.

Scholte (2000) argues that the popularity of the new terminology most likely reflects a widespread intuition that social relations have acquired a new character (i.e., a new historical condition has emerged). If new terminology spreads so far and attracts so much attention, might it not be more than a synonym for pre-existent vocabulary. He distinguishes five common conceptions of globalisation (see also Table 1.2).

- Internationalisation: 'global' is used to describe cross-border relations between countries and 'globalisation' designates a growth of international exchange (e.g., of capital, people, messages, ideas) and interdependence.

- Liberalisation: globalisation is seen as a process of removing governmentimposed restrictions on movements between countries in order to create an open world economy (i.e., international economic integration).

- Universalisation: here 'global' means 'worldwide' and globalisation is explained as the process of spreading various objects and experiences to people at all corners of the world.

- Westernisation or modernisation: globalisation is seen as a dynamic whereby the social structures of modernity (capitalism, rationalism, industrialism, bureaucratism, etc.) are spread throughout the world, normally destroying pre-existent cultures and local self-determination in the process.

- Deterritorialisation or the spread of supraterritoriality: globalisation entails a reconfiguration of geography, so that social space is no longer wholly mapped in terms of territorial places, territorial distances and territorial borders (i.e., trans-world or trans-border interactions emerge).

Accordingly, Scholte (2000) states that only the last of these five approaches offers the possibility of a clear and specific definition of globalisation. Although the other four approaches are all viable in their own terms, they do not 
highlight a new historical condition. They all presume that the map of society is solely and completely territorial, while the notion of supraterritoriality refers to a novel transformation regarding the nature of social space. The concept of deterritorialisation provides a way into appreciating what is 'global' about globalisation. Globality marks a distinct and mostly new kind of space-time compression; in transborder interactions, place is not territorially fixed, territorial distance is covered in effectively no time, and territorial boundaries present no particular barrier. This increasing globality can be observed in almost all aspects of our social life, including communications, travel, production, markets, money, finance, organisations, military, ecology, law, consciousness and health. It is important to note, however, that Scholte refers to a 'relative' deterritorialisation; territory still matters, of course, but global relations have substantially (rather than totally) transcended territorial space.

Table 1.2: Different approaches to globalisation (based on Scholte (2000)).

$\begin{array}{ll}\begin{array}{l}\text { Approach } \\ \text { Internationalisation }\end{array} & \begin{array}{l}\text { Example deftinitions } \\ \text { Globalisation is, briefly, the intensification of economic, political, social } \\ \text { and cultural relations across borders (Holm \& Sorensen, 1995). }\end{array} \\ \text { Liberalisation } & \text { Globalisation has become a prominent catchword for describing the } \\ \text { process of international economic integration (Sander, 1996). } \\ \text { Universalisation } & \begin{array}{l}\text { Planetary synthesis of cultures (Reiser \& Davies, 1994). } \\ \text { Westernisation/ } \\ \text { modernisation }\end{array} \\ \text { Geterritorialisation } & \text { Hollywood and CNN (Schiller, 1991). } \\ & \begin{array}{l}\text { Globalisation can be defined as significant global connection, with a } \\ \text { corresponding diminution in the significance of territorial boundaries and }\end{array} \\ & \text { state structures (Bretherton, 1996). } \\ & \text { Globalisation is a process (or set of processes), which embodies a } \\ \text { transformation in the spatial organisation of social relations and } \\ \text { interactions (Held et al., 1999). }\end{array}$

In order to avoid simplification of the complexities involved in approaching globalisation, Rennen and Martens (2003) describe globalisation by means of a timeline identifying key historical landmarks of economic, political, technological, social-cultural and environmental developments that have pushed the process of globalisation further. They argue that, taking the extensiveness, intensity, velocity and the impact of contemporary globalisation into account, it is legitimate to assume that the processes underlying it have the potential to change over time, in a non-linear way, characterised by periods of

\footnotetext{
1 In territorial geography, relations between people are mapped on the earth's surface and measured on a three-dimensional grid of longitude, latitude and altitude. Territorialism implies that social space is wholly organised in terms of units such as districts, towns, provinces, countries and regions (Scholte, 2000).
} 
progress, stabilisation, and temporary decline. Before the 1960s, globalisation was intrinsically an economic, political and technological process. However, this approach refers to the emergence of globalisation and not to its current state. Since the 1960s, social, cultural and environmental developments also became important factors that co-shaped globalisation. Hence, they define contemporary globalisation as 'an intensification of cross-national cultural, economic, political, social and technological interactions that lead to the establishment of transnational structures and the global integration of cultural, economic, environmental, political and social processes on global, supranational, national, regional and local levels'.

To conclude, it cannot be denied that what once was characterised as an economic, technological and political driven phenomenon, is also taking place in a social-cultural and environmental setting. There is more and more agreement on the fact that globalisation is an extremely complex phenomenon; it is the interactive co-evolution of multiple technological, cultural, economic, institutional, social and environmental trends at all conceivable spatiotemporal scales. Nowadays, hardly anybody seems to deny the phenomenon of globalisation as such. However, it is often a vague concept due to lack of clarity, imprecision and inconsistencies in respect to definitions and approaches. Based on the work by Scholte (2000), Rennen and Martens (2003), and Held et al. (2000), we define globalisation as 'a process characterised by a growing intensity, extensity and velocity of institutional, economic, social-cultural and ecological interactions, resulting in transborder processes and effects' (Huynen et al., 2005b). This definition is in line with the view on globalisation in terms of a relative diminution in spatial boundaries (deterritorialisation), and it explicitly acknowledges the multiple dimensions involved. Consequently, the term globalisation can be viewed as a collective label, instead as one giant process in itself (Martens \& Rotmans, 2002).

\subsection{SCOPE AND OBJECTIVES}

Promoting good health is one of the key elements in achieving a sustainable form of globalisation, including the need to identify and address both the risk and opportunities provided to us. The resulting call for a global, integrated and forward-looking approach to health becomes stronger. Policymakers worldwide are challenged to avert possible negative health impacts of current and future globalisation. However, there is also a growing need to manage this process in such way that local benefits will be derived specifically in terms of population health (e.g., reducing current health inequalities and ensuring intergenerational equity).

Global health research addresses the ways in which globalisation is impacting on health determinants and health outcomes (Lee, 2003). This is a rather new 
and challenging research field. Current literature shows a prevailing lack of consensus about the future health effects of globalisation. As a result, it is difficult for policymakers and scientists to make the link between the globalisation process on the one hand and a diversity of (possible) health impacts on the other hand.

An important objective of this Ph.D. Thesis is to provide a better understanding of the possible (future) health implications of the globalisation process, including global environmental change. This will aid both scientists and policymakers to work through the wide variety of interactions between global developments and human health, dealing with critical uncertainties and plurality along the way. Parts of this research are primarily future-oriented. Scenario analysis is used to explore the health effects of different future globalisation pathways. Past developments in our health, conceptual modelling, epidemiological insights and the variety of views abound in current literature are used to substantiate the logic in these alternative explorations of our future health.

This Ph.D. Thesis aims to answer the following central question: How will future health evolve given anticipated globalisation trends? In order to address this question, the following research objectives are identified:

1. To assess past developments in population health, both in the developed and developing world.

2. To develop an integrated framework to analyse the consequences of globalisation for population health, accounting for plurality along the way.

3. To explore future health given anticipated globalisation pathways and associated developments in important health determinants, both in the developed and developing world.

4. To explore the (future) health impacts of global environmental change, focussing on global climate change and global biodiversity loss.

Furthermore, the methodological approach underlying this study is meant to contribute to the ongoing discussions and development of methods regarding the analysis of (future) health in a globalising world.

\subsection{OUTLINE}

This Ph.D. thesis is structured as follows. The next Chapter describes our methodological framework, which is based on a systems-approach towards both globalisation and population health.

In Chapter 3, a broad overview is given of the past developments in health using the framework of the epidemiological transition theory. A descriptive analysis of the changes observed in the Netherlands and in Peru provides insights into the different transition experiences of these two countries. 
Subsequently, the lessons learned from the epidemiological transition are discussed from a systems perspective.

Chapter 4 presents an integrated conceptual framework for the health implications of globalisation, building on a comprehensive analysis of relevant literature regarding population health models, the globalisation processes, and the various perspectives that abound on the effects of globalisation on population health. Accordingly, Chapter 5 explores future developments in population health resulting from different globalisation pathways, for both the developed and developing countries, by combining insights from past health transitions, nine selected global scenarios studies and the developed conceptual framework.

The next two Chapters focus on the health impacts of global environmental change. Chapter 6 investigates the climate change-induced changes in temperature-related mortality in the Netherlands. The relationship between mortality and (extreme) temperature is assessed using an epidemiological approach (time-series analysis and episode analysis). Based on the resulting quantitative exposure-effect relationships, the implications of future climate change are assessed, acknowledging the uncertainties involved. Chapter 7 quantitatively assesses the association between biodiversity loss and health by means of a regression analysis using country level indicators for biodiversity loss, population health and socio-economic development. It also discusses the limitations of such a reductionist approach.

Finally, Chapter 8 recapitulates the main conclusions drawn in this Ph.D. Thesis, provides a brief methodological reflection, and offers an outlook to future work. 


\section{Chapter 2}

METHODOLOGICAL FRAMEWORK 


\section{METHODOLOGICAL FRAMEWORK}

The health impacts of globalisation pose new challenges to scientists and policymakers. How can we address such a broad issue, encompassing debated relationships between multiple factors operating at different positions in the causal chain? This Chapter argues that the characteristics of both population health as well as the globalisation process demonstrate that a system-based approach is required. Integrated Assessment (IA) provides such an approach, and associated tools. The Chapter concludes with the research approach of this Ph.D. Thesis.

\subsection{GLOBALISATION AND POPULATION HEALTH: THE COMPLEXITIES INVOLVED}

As discussed in the previous Chapter, the causality of human health is multifactorial and many population health problems are invariably embedded within a global context. McMichael (1999), for example, argues that a global approach towards population health and epidemiology should not ignore the importance of individual level proximal risk factors, but should indicate the importance of studying these proximal causes in their broader context. In line with this, Colwell (2004) argues that the health issues of the 21st century should be placed within the web of life, recognising the linkages between our health and processes that operate at the global scale. As our attention moves upstream in the causal chain of health determinants', there is an increasing interest in multilevel- and systems-approaches (McMichael, 1995, 1999; Pearce, 2004; Pearce \& Merletti, 2006). Hence, a growing number of health researchers (Albrecht et al., 1998; Colwell, 2004; McMichael, 2005; Pearce \& Merletti, 2006; Wilcox \& Colwell, 2005) argues that the health of a population can- or must- be viewed within the broader system of health determinants. Populations are not simply the collection of individuals, but are shaped by, and shape, the systematic context in which they operate (Pearce \& Merletti, 2006). Risk factors for disease do not operate in isolation, but occur in a particular population context. Upstream forces play an important role in global health research (Sreenivasan \& Benatar, 2006). These upstream or contextual factors may have large impacts, but their effects are non-linear and less-predictable (Philippe \& Mansi, 1998). Various terms have been used to describe such broader approaches to population health, such as eco-epidemiology (Ladd \& Soskolne, 2008; Martens, 1998a; Soskolne \& Broemling, 2002; Susser \& Susser, 1996), ecological perspective on health (McLaren \& Hawe, 2005), social-ecological

I See also Chapter 4 for an explanation of the different hierarchical levels of health determinants in the causal chain. 
systems perspective on health (McMichael, 1999), and biocomplexity approach to health (Colwell, 2004; Wilcox \& Colwell, 2005).

The majority of literature addressing population health as an interacting system of many different factors concerns research into communicable diseases. Disease transmission depends on contextual factors such as environmental change and cultural practices affecting landscapes, communities and population densities. These factors, in turn, interact with host-pathogen biology via evolutionary ecological processes to contribute to the (re)emergence of communicable diseases (Kapan et al., 2006). Parkes et al. (2005), among others, plea for new system-based approaches to address communicable diseases. They argue that the worldwide (re)emergence of infectious diseases (e.g., SARS, Nipah virus, Lyme disease, HIV/AIDS, and malaria) demonstrates that 'the rate and scale of global change in agriculture, trade, demographics, species translocations and invasions, microbial adaptation, and other complex factors have outstripped our ability to understand and respond to emerging infectious diseases', and 'expose serious limitations of approaches that fail to engage with the wider contexts from which infectious diseases emerge'. For example, the risk of highland malaria moving to higher altitudes depends on the interplay between regional climate change, land use change, population movement, agricultural practice (e.g., pesticide use, irrigation systems), public health programmes (e.g., monitoring and treatment) and socio-economic status (Hales \& Woodward, 2003; McMichael \& Woodruff, 2005). The dynamic interaction of these various factors is just one of the many examples of the broader population context in which infectious diseases develop (Albrecht et al., 1998; Kapan et al., 2006).

With regard to epidemiological research into non-communicable diseases, applications of a system-based approach are rare. However, the social and economic conditions at the population level can often explain the differences in exposure to risk factors. Individual lifestyle, for example, can only be understood in the historical, cultural and social context in which it occurs (Pearce \& Merletti, 2006). Albrecht et al. (1998) apply a complexity approach to coronary heart disease in the Australian coalfields, resulting in an improved understanding of the dynamic interplay between industrial history, heart disease risk factors, gender promotion, and community responses to both the health problem and the health promotion campaigns.

The increasing awareness that a more system-based approach towards (global) population health is required, is in line with the more general 'complexity turn' in science. The recognition that many issues should be studied as a whole instead of studying its different parts separately played an important role in the development of complexity theory (Box 2.1). Table 2.1 provides an overview of the most important differences between the (traditional) Newtonian and complexity paradigms. 
Box 2.1: From Newtonian to complexity (adapted from Amelung (2006)).

For centuries, the dominant scientific worldview has been a mechanistic image of a static, repetitive, predictable, linear, and clockwork universe. This so-called Newtonian approach to science- named after Isaac Newton- seeks full knowledge of a system via analysis or 'reduction' of the system to its parts followed by study of the parts. In the 20th century, however, elements of Newtonian science were being dismantled. Einstein changed our Newtonian perception of time (i.e., time is invariant, infinitely divisible into space-like units, measurable in length, expressible as a number and reversible) by showing that that there is no fixed or absolute time independent of the system to which it refers. Thermodynamics showed that there is an irreversible flow of time; the arrow or flow of time results in futures that are unstable, relatively unpredictable and characterised by various possibilities (Prigogine, 1997). Scientists continued to find physical phenomena that were not amenable to the traditional Newtonian scientific method. This failure of the Newtonian approach - based on reductionism, linear relationships, negative feedback and a tendency towards equilibrium- to be generic, resulted in a need for a new research paradigm. The physical sciences began to distinguish between 'linear' and 'non-linear' phenomena (see e.g., Prigogine \& Stenger (1984)). In linear systems, causes lead to known effects in a predictable and repeatable manner; systems can be disassembled to understand the behaviour of their constituent elements and then reassembled, in a clockwork fashion, to model the behaviour of the whole system under differing conditions. Non-linear systems, however, are composed of numerous elements that interact locally according to simple rules, but the resulting internal dynamics of such a system create complex, disproportional and unpredictable outcomes. In general, these non-linear phenomena clearly reflect the uncertainty and complex nature of the majority of social and natural processes that we are trying to get our head around.

The development of chaos and complexity theory spurred a revolution in the natural sciences. Lorenz (1963) discovered that even simple deterministic systems could exhibit nonlinear behaviour. The development of the chaos theory involved rejecting the Newtonian notion that only large changes in causes can produce large changes in effects. Infinitesimally small differences in initial conditions were found to produce hugely divergent evolutions of the system (Gleick, 1987; Prigogine \& Stenger, 1984). A characteristic that became known as the butterfly effect: 'a butterfly stirring the air today in Peking can transform storm systems next month in New York' (Gleick, 1987). Paradoxically, the same positive feedback mechanisms that cause butterfly effects, emblems of volatility, can also be responsible for inertia. Hypersensitivity to initial conditions implies path dependency, which sometimes produces a strong network of mutually reinforcing relationships that endure long after the initiating conditions have been superseded: a 'lock-in' effect (Waldrop, 1992). Nonlinearities occur in complex systems of many kinds, ranging from fluid systems to the global weather system and the economy. In many of these systems, long periods of relative stability (phases) are interrupted by bursts of turbulence (phase shifts) that mark periods of accelerated evolutionary change (Faulkner, 2001).

Complexity is the study of emergent, dynamic and self-organising systems that interact in ways that heavily influence the probabilities of later events. Such complex systems encompass many entities interacting with each other, but the richness of these interactions allows the system as a whole to undergo self-organisation. As a result, complex systems have the ability to adapt and co-evolve as they organise through time. They are also characterised by non-linearity, positive feedback loops and points of burification (Waldrop, 1992). 
Table 2.1: Different perspectives on science: the Newtonian paradigm versus the complexity paradigm.

\begin{abstract}
Newtonian paradigm
Reductionism: Developing an understanding of a system's constituent parts (and their interactions) is the best way to develop an understanding of the system as a whole.

Systems respond in a predictable way according to universal laws.

Linearity: A direct and proportional connection can be established between each cause and effect.
\end{abstract}

Uncertainty is a symptom of bad science and needs to be reduced.

Deterministic, one possible future.

Systems tend towards equilibrium and are driven by negative feedback.

Non-historical (time-reversible).

\section{Complexity paradigm}

Holism/contextuality: Complex systems should be studied as a whole; they can have emergent properties that are not explainable from the sum of their (reductionist) parts.

Systems respond in an unpredictable way.

Non-linearity: A small perturbation may cause a large effect. This is often called a 'butterflyeffect' (see also chaos theory). Tipping points may be reached when the system passes a particular threshold.

Uncertainty is inherent to complex systems and needs to be acknowledged.

Non-deterministic/stochastic, multiple futures are possible.

Systems are inherently unstable and positive feedback-driven processes are common. Path-dependence (time-irreversible): complex systems are dynamical systems- they change over time, and prior states may have an influence on present states.

Following its growing influence in the natural sciences, complexity theory has 'begun to spill onto the edges of the social sciences as well' (Urry, 2005b). Various scientists studying the processes of globalisation- often implicitly- draw upon concepts and ideas from the field of complexity theory (see e.g., Amelung (2006); Knorr Cetina (2005), Urry (2003, 2005a), and Castells (1996)); the many interacting processes of globalisation are perceived to adapt and co-evolve as they organise through time, which results in inherent uncertainty, and multiple futures.

Hence, the global context of population health comprises various systems, operating at various levels or scales, and each constitutes the environment for the other. Although complexity theory has had relatively little influence in the fields of population health and epidemiology (Pearce \& Merletti, 2006), the above shows that the past few years have witnessed a growing recognition of the multidimensional and multilevel causation of population health and the importance of a holistic system-based approach. As Soskolne et al. (2007) state, we 'must embrace greater complexity' as 'the traditionally used, reductionist, linear approaches are inferior for understanding the interactive webs that are critical for sustainable development and for the health and well-being of future generations'. 
Interdisciplinary research approaches can contribute to the improved understanding of global health, using conceptual frameworks and theoretical bases from all relevant disciplines involved. However, in order to fully address complex, interconnected, system-based health topics, transdisciplinary approaches are required to facilitate newer modes of science, that transcend the disciplinary boundaries (Soskolne et al., 2007) (see also Table 2.2). Transdisciplinary approaches to human health can be defined as approaches that integrate the natural, social and health sciences in a humanities context, and in so doing transcend each of their traditional boundaries. Emergent concepts and methods are the hallmark of the transdisciplinary effort (Soskolne \& Bertollini, 2002; Soskolne, 2003).

Table 2.2: Taxonomy of cross-disciplinary research (Rosenfield, 1992).
Level
Description
Multidisciplinarity A common problem or set of problems is analysed by each discipline independently and the results are usually brought together at the end.
Interdisciplinarity Different disciplines work jointly, but still from disciplinary-specific basis (i.e. using their own concepts, techniques and skills) to address a common problem.
Transdisciplinarity Researchers work jointly using a shared conceptual framework drawing together disciplinary-specific theories, concepts and approaches to address a common problem.

To conclude, the complexity involved in assessing the health impacts of globalisation is characterised by:

- Holism and systems thinking: The linkages between the processes of globalisation and population health are modified by multiple factors, which cannot be studied in isolation from each other. The underlying processes interact at various scales.

- Uncertainty and plurality: The impacts of globalisation on health depend on the interplay between the many (global) developments, which together form the broader context of population health. The underlying processes are not fully understood, and might behave in unpredictable ways. Due to this inherent uncertainty, subjectivity and plurality are introduced.

- Inter- and transdisciplinarity: The issue at stake lies across, or at the intersection of many disciplines. In other words, the institutional, economic, social-cultural and environmental dimensions need to be included in the analysis, moving beyond multidisciplinary research to inter- and transdisciplinary approaches. 


\subsection{INTEGRATED ASSESSMENT}

The previous section argued that in our effort to asses the health impacts of globalisation, we have to be aware of the limitations of the traditional reductionist approaches (Albrecht et al., 1998; Pearce, 2004; Pearce \& Merletti, 2006); population health and globalisation cannot be disassembled to their constituent elements and then reassembled in order to develop an understanding of the system as a whole. A more holistic and integrated perspective has considerable potential to assist researchers to address global health issues. In view of the main research question of this study, a systembased approach implies a lower emphasis on prediction, but an accompanying greater emphasis on understanding of the processes involved, acknowledging inherent uncertainties, and exploring alternative health futures.

The field of IA provides such an alternative, holistic approach. A number of authors (Gough et al., 1998; Harremoës \& Turner, 2001; Harris, 2002; Rotmans \& Dowlatabadi, 1998; Rotmans, 1998, 1999; Weyant et al., 1996) set about scoping and defining the field of Integrated Assessment. Rotmans (1998) defines IA as 'a structured process of dealing with complex issues, using knowledge from various scientific disciplines and/or stakeholders, such that integrated insights are made available to decision makers.' Thus, IA is not just another type of policy-support; it addresses only complex issues, which it aims to understand and structure. Holism, uncertainty, plurality, and inter- and transdisciplinarity are among the key challenges that IA aims to address:

- Holism and systems thinking: IA emerged as a new field in global change research because the traditional disciplinary approach was unable to put global change issues in the broader context of interacting social-cultural, economic, and environmental conditions and developments (Rotmans, 1998). According to Gough et al. (1998), IA is a method for assessing complex systems, its strength lying in making the multiple interactions that exist between natural, economic and social system explicit. Hence, taking a system-based perspective is at the core of IA.

- Uncertainty and pluralism: It is commonly accepted that 'a useful IA should be able to cope with a plurality of perspectives on a particular issue' (Gough et al., 1998). This requirement stems directly from an essential feature of many systems: uncertainty. Lack of knowledge is one obvious source of uncertainty, but there are also fundamental uncertainties that cannot be reduced by doing more research (Rotmans, 1998). The omnipresence of uncertainty in the complex systems that IA aims to structure and assess allows for different valid views on the essence and functioning of these systems. Van Asselt $(2000,2006)$ argues that plurality plays an important role in IA, because uncertainty legitimates different perspectives, such as different disciplinary perspectives, actor/stakeholder perspectives, or world views (e.g., norms and value systems). 
- Inter- and transdisciplinarity: A common feature of complex issues is that they do not respect disciplinary boundaries. Due to its focus on holism and the recognition of multiple valid perspectives, IA requires interdisciplinary and transdisciplinary approaches, incorporating the institutional, economic, social-cultural and ecological dimensions involved. Hence, IA is an issueoriented research approach that knits together pieces of knowledge from many disciplines (Rotmans \& Dowlatabadi, 1998) in order to focus holistically on the issue at hand. One of the basic ideas behind IA is to combine, interpret and communicate knowledge from different scientific disciplines (Valkering et al., 2006) and from different stakeholders. Parkes et al. (2005) distinguish horizontal and vertical integration of knowledge and viewpoints. Horizontal integration defined as integration across knowledge perspectives, such as disciplines or sectors. Vertical integration means integration among different types of knowledge users, and may involve perspectives from academics, as well as local communities and cultures, and NGO staff, for example. Although stakeholder participation is not a necessary prerequisite, it is often an important part of IA (Valkering et al., 2006). While often rewarding in the end, the actual process of building bridges across disciplinary divides is typically very timeconsuming (Rotmans \& Dowlatabadi, 1998). IA can, however, also be the work of individuals. As Harris (2002) puts it: 'it still requires a few people to sit down and go through the mountains of literature, seeking the foundations of edifices and the outlines of new architects' plans.'

The methods that were developed and applied in the context of IA projects are commonly grouped together into two categories: analytical tools and participatory methods2. The most widely used analytical methods are model analysis and scenario analysis. These methods are considered more mature than the participatory methods: they are reasonably well defined, and there is at least a workable amount of common understanding of their scope, advantages and disadvantages (Rotmans, 1998). Not only is there more experience with the use and application of these methods in IA contexts, they are also more widely accepted and used as legitimate scientific tools in disciplinary, 'normal' science than participatory methods. The selection of a specific method for performing an integrated assessment highly depends on the context of the assessment. Additionally, an integrated assessment is best supported by a combination of tools (see e.g., Valkering (2006) and van Asselt (2001)), for example:

- Participatory processes can contribute to model building by revealing different perspectives on model structure or key components.

- Participatory processes can provide input for scenario development by revealing different perspectives on vital uncertainties and possible futures.

${ }^{2}$ For a more comprehensive description of IA-methods we refer to Rotmans (1998), van Asselt et al. (2001) and Valkering et al. (2006). 
- Scenarios can be used as input for integrated models.

- Scenarios can be used as input for participatory processes.

- Integrated models can be used as input for participatory processes.

- Integrated models can provide input or validity check in scenarios (e.g., global scenarios) by, for example, defining realistic ranges for key aspects of scenarios.

\subsubsection{Integrated Assessment models}

Models were arguably the first tools that were used in integrated assessments. One of the earliest examples of such models was the one underlying the famous 'Limits to Growth' report to the Club of Rome by Meadows et al. (1972). In a limited number of equations, the authors tried to combine knowledge on a number of key issues such as demography, economy and resource use to explore the future of mankind's interaction with nature and the environment. Later in the 1970s, scientists exploring the issue of acidification took a similar approach in institutes such as the International Institute for Applied Systems Analysis (IIASA) and the Dutch National Institute of Public Health and the Environment (RIVM) (Rotmans \& Dowlatabadi, 1998).

IA models are simplified representations of complex real-world phenomena. They combine knowledge elements from various disciplines in an analytical framework, to assess the socio-economic and environmental consequences of human activities. In general, IA models try to describe quantitatively as much as possible the cause-effect relationship of a phenomenon, and the crosslinkages and interactions between different contextual circumstances and processes (Martens, 2006; Rotmans, 1998). They are never intended as 'truth machines' that perfectly replicate systems or predict the future. Rather, they are used for structuring information, and for exploring interactions and sensitivities (Rotmans, 1998; Valkering et al., 2006; van Asselt et al., 2001).

In modelling population health dynamics, regression techniques are usually used to explore the relations between health determinants, like literacy rate, income status, nutritional status, water supply and sanitation, education and medical services, and the health status measured in, for example, healthy life expectancy. However, these regression techniques can only give some suggestive evidence on the causes of population and health changes. Statistical models to estimate future health levels are based on extrapolation of past and current data. They usually are rather static in terms of specifying the dynamics behind changing health patterns. Therefore, there is a need for integrated approaches that take into account multiple risk factors at various levels in the causal chain. Such an integrated approach cannot be used in the clinical area on an individual basis, but is appropriate at the population level (Martens, 2006). 
In IA, modelling primarily refers to mathematical simulation models, which work on the basis of quantitative information and requiring quantitative data inputs as they provide quantitative outputs. However, one of the first- but often hidden- steps in building a quantitative simulation model is the formulation of a conceptual model. This is a qualitative model that helps to understand and structure real-world systems and processes. Integrated conceptual models provide a structured representation of a system and they are not limited by the availability of quantitative data. Especially in the phase of issue- or problemframing, the development of a conceptual model improves the integrated understanding of all key components and processes involved. In the case of complex systems, this process should explicitly acknowledge the uncertainties and the consequent plurality involved. In this sense, developing a conceptual framework is a means in itself. Additionally, well-described conceptual frameworks provide a sound basis for further research, whether this consists of carrying out a scenario analysis, organising a participatory process, building a quantitative model, or a combination of these tools.

\subsubsection{Integrated scenario analysis}

The fact that the future can never be known with certainty does not mean that little of value can be said about it (Butler, 2005). Thinking about what happens in the future provides a means to share our understanding about particular systems and situations, and the concerns we have in relation to these. Given the uncertainty about which of these complex issues may actually come to pass, it is useful to explore alternative options. Scenarios analysis is a means for structured thinking about the future (Rothman, 2006).

Scenarios can be defined as descriptions of journeys to possible futures that reflect different assumptions about how current trends will unfold, how critical uncertainties will play out and what new factors will come into play (UNEP, 2002). A key point in this definition is that a scenario of the future includes not only the state of the system at the end of the scenario period, i.e., the future vision, but also the path from today to the specified future (Rothman, 2006). Scenarios describe hypothetical future pathways that consist of states, events, actions and consequences that are causally linked. In other words, scenarios are described as plausible but simplified descriptions of how the future may develop, according to a coherent and internally consistent set of assumptions about key driving forces and relationships (Swart et al., 2004). As such, scenario analysis is an often-used tool to deal with uncertainties by making the different legitimate interpretations explicit; scenarios are usually developed in sets, in which each scenario explores the implications of different assumptions concerning uncertain relationships or uncertain developments in key drivers. 
Scenario analysis has evolved significantly over the past decades. In the early days, scenarios were used primarily as planning and forecasting tools to display a rather mechanistic and deterministic worldview. In traditional types of scenario analyses, there is considerable confidence in the predictability of the future. Based on information about the current situation and current trends, projections for the future state or development are constructed. Such projections are typically referred to as 'business as usual' (BAU) scenarios. Uncertainties were presumed to originate from 'noise' and lack of knowledge. The complexity paradigm challenges the traditional approach of exploring the future. IA, being an issue-centred rather than disciplinary approach, challenges the BAU-type of scenario analysis; it rejects the disciplinary way of making projections and proposes an integration of projections and insights into coherent integrated scenarios. As such, the value of scenario studies to explore possible future events and provide sound policy-relevant guidance for decision-makers is increasingly and widely recognised; scenario analysis is a well-established response to uncertainty about what the future will bring.

In IA, scenarios are perceived as powerful exploratory tools. They do not predict the future, but rather they paint pictures of possible futures and explore the various outcomes that might result if certain basic assumptions are changed (UNEP, 2002). In addition, scenario analysis has moved beyond merely fulfilling a decision-support function to supporting a more open form of exploration. According to Rotmans (1998), scenario analysis can be used to articulate key assumptions and expand our thinking beyond the conventional. Over the years, a variety of scenarios have been developed, differing in terms of subject (issue-based, area-based, institution-based), and temporal and spatial scales (see e.g., van Notten et al. (2003) or Greeuw et al. (2000) for an overview). Typical integrated scenarios are issue-based or area-based and employ a time horizon of a few decades. Some scenarios look forward; they begin at present day, pose one or more 'what-if' type questions, and then proceed to explore the ensuing futures. Other scenarios begin with an explicit image of a future state and then try to work out how we might reach that state starting from the present situation by posing 'how-could' type of questions (Rothman, 2006). A number of spatial scopes have been used for scenario development, ranging from the local scale, to the continental and global scales. Scenarios can be developed using qualitative and quantitative representations. These should be seen as complementary. Qualitative scenarios can explore relationships and trends for which few or no numerical data are available, including shocks and discontinuities. They can more easily incorporate human motivations, values and behaviour and create images that capture the imagination of those for whom they are intended (Rothman, 2006; UNEP, 2002). Alternatively, quantitative scenarios involve an interaction between narratives and computerized models. Such modelled scenarios apply key assumptions to 
quantitative data to produce quantifiable explorations of elements that will exist at a given point of the future (Butler, 2005); the effects of changes in assumptions can then be easily checked, pointing to important uncertainties (Rothman, 2006; UNEP, 2002).

\subsubsection{Participatory methods}

Participatory methods - such as policy exercises and focus groups - are linked to the emerging paradigm of 'postnormal science' (Funtowicz \& Ravetz, 1994). The scientific movements of postmodernism and social constructivism have challenged the monopolistic position of science in the production of knowledge. It is argued that the quality of research can be improved by involving relevant stakeholders, because these can contribute practical knowledge and experience, as well as a range of different perspectives (van Asselt \& Rijkens-Klomp, 2002). Funtowicz and Ravetz (1994) and Ravetz (1999) see the involvement of an 'extended peer community', consisting of all relevant stakeholders, as a superior form of quality control in the context of complex issues. Although this more modest view of science's role has gained ground over the last few decades, it still meets with considerable opposition and hostility. Many researchers hesitate to accept the full implications of the existence of fundamental uncertainties, being that 'searching for the truth or for universal answers' must be abandoned (Rotmans, 1999). The set of participatory methods that is used in IA is not clearcut, and the methods themselves are not clearly defined (see Box 2.2).

Box 2.2: Overview of participatory methods (van Asselt \& Rijkens-Klomp, 2002).

The five participatory methods that have been used in IA are focus groups, participatory modelling, scientist-stakeholder workshops, scenario analysis, and policy exercises. They all help assessors in structuring, eliciting tacit knowledge about and identifying perspectives on the complex issue being studied, albeit in different ways.

- Focus groups consist of a limited number of stakeholders who have structured moderated discussions to elicit preferences, opinions and viewpoints.

- The method of participatory modelling takes the additional step of allowing stakeholders to explore, while they discuss, the implications of their ideas by formalising them in a model.

- In scientist-stakeholder workshops, stakeholders aid scientific experts by discussing scientific findings and helping to identify key research priorities and to formulate a research agenda.

- To have stakeholders identify key issues is also a main aim of scenario analyses, but in this case the participants take the additional step of constructing plots for the future development of these issues. As a next step, these plots can be knitted together into a set of full-blown scenarios that explore a range of possible futures.

- In policy exercises, a heterogeneous group of stakeholders synthesises the complex issue at hand. Subsequently, the participants assume different roles to simulate a decision-making process in order to explore the dynamics of such a process. 


\subsection{RESEARCH APPROACH}

As a system-based interdisciplinary approach is required to assess the (future) health implications of globalisation, this study uses IA as its methodological backbone, in combination with epidemiological approaches.

Although primarily future oriented, this Ph.D. Thesis starts with looking back to the past developments in population health. The reasons for our study of the past epidemiological transition are two-fold. First, scenario analysis should be based on insights from past developments in health. Second, if a system-based approach towards population health is required, the epidemiological transition theory must also be viewed from a systems perspective. Albrecht et al. (1998) argue that researchers seeking a generic perspective of health complexity can draw on existing integrated approaches such as the epidemiological (or health) transition theory. When the epidemiological transition was first published by Omran (1971), it was generally assumed that all counties would more or less follow the same transition pathway. Later, it was acknowledged that not all populations follow the same trajectory. This becomes critically evident when comparing the health changes in developed countries with the changes observed in developing countries, resulting in the formulation of different transition models. For this reason, we first describe the past epidemiological transition in the Netherlands and in Peru. This descriptive analysis results from several iterations going back and forth between reviewing the epidemiological transition theory and assessing the past health developments in these two countries. Accordingly, the lessons learned about the epidemiological transition are evaluated in view of the systems approach adopted in this Ph.D. Thesis. The resulting insights also provide a basis for our exploration of future health in the developing and developed world.

'Global health' is a rather new research field. Hence, a clear conceptualisation of the system under investigation is required. The first Chapter already defined the concepts of globalisation and population health. Building on these definitions, we develop a conceptual framework for the health implications of the globalisation processes, which is rooted in an integrated approach towards both globalisation and population health. This process includes the following steps: 1) reviewing existing health models and identifying their strengths and weaknesses 2) defining the concept of population health and identifying/structuring its main determinants; 3) defining the concept of globalisation and identifying its main features; and 4) constructing the conceptual model for globalisation and population health. The extensive literature review underlying our conceptual framework reflects the diversity of views that abound in various relevant disciplines.

Based on the lessons learned from the past epidemiological transition theory and the conceptual framework, an integrated scenario analysis explores the 'future health transition' according to a variety of globalisation pathways. This 
analysis builds upon nine selected existing global scenario studies. First, the 'health-dimension' in these existing scenarios studies is analysed. Furthermore, we assess whether the scenario studies included other developments relevant for future health. In order to provide examples on how future health can be incorporated in existing scenarios, several possible, but imaginary, health futures are sketched- the 'age of emerging diseases', the 'age of medical technology', the 'age of sustained health', and the 'age of chronic diseases for non-western countries'. These future health images build upon past and current transition stages, on existing views about future health in current literature, and on possible early signs observed within our society. Accordingly, the institutional, social-cultural, economic, and environmental developments described in existing scenario studies are linked to these different health futures. The analysis provides an integrated outlook on fundamentally different health futures for both the developed and developing worlds.

Besides these qualitative approaches, this Ph.D. Thesis takes a quantitative approach in addressing the health impacts of global environmental change. First, we focus on the health impacts of climate change in the Netherlands, and the changes in temperature-related mortality in particular. This research starts with the study of temperature and mortality in the Netherlands, using daily temperature and mortality data for 1979-1997. The effects of temperature on mortality are rather direct and standard epidemiological approaches are widely accepted: we conduct a time series analysis and, furthermore, episode analysis to asses the effects of extreme heat or cold periods. Accordingly, we explore future climate change-induced temperature-related mortality in the Netherlands, making several assumptions (e.g., acclimatisation). In this study, epidemiological results are combined with recently developed Dutch climate change scenarios. The analysis does not pretend to yield precise results or definitive conclusions about the temperature-related mortality effects of climate change in the Netherlands. Its main objective is to provide an order-ofmagnitude estimate of the effects, acknowledging the uncertainties involved and communicating the assumptions made in an explicit way.

We apply a rather straightforward approach in our study of the health effects of biodiversity loss. We explore the association between health and biodiversity loss by means of regression analysis on a global scale, with control for confounding by socio-economic developments. For this, we selected several indicators for human health, biodiversity loss, and socio-economic development.

To conclude, the methodological approach underlying this study, hopefully, contributes to the further development of global health research. In the final Chapter, we will reflect upon the identified methodological challenges (holism and systems thinking; uncertainty and plurality; inter- and transdisciplinarity) within our research. 


\section{Chapter 3}

\section{PAST DEVELOPMENTS IN OUR HEALTH: THE EPIDEMIOLOGICAL TRANSITION}

Section 3.3 is based on:

- Huynen, M.M.T.E., Vollebregt, L., Martens, P., \& Benavides, B.M. (2005). The epidemiologic transition in Peru. Pan American Journal of Public Health, 17(1), 51-59. 


\section{Past DEVElopMents IN OUR HEALTH: THE EPIDEMIOLOGICAL TRANSITION}

Although our main research question is primarily future oriented, this Chapter looks back to the preceding developments in population health. The past developments in our health encompass several related processes such as the increase in life expectancy, the decline in mortality rates, the changing configuration of causes of death, the changing character of morbidity, and the continuing developments regarding the provision of health services. Global life expectancy at birth more than doubled in the past century to about 66 years. At present, life expectancy is more than 75 years in the developed world and 64 years in less developed countries (however, life expectancy in the least developed regions is still as low as 53 years) (UN, 2007b). The proportion of deaths due to infectious diseases has decreased, while the relative importance of chronic diseases increased.

Box 3.1: What's in a name: epidemiological or health transition?

Some conceptual confusion might arise from interchanging the terms 'epidemiological transition' and 'health transition'. In 1971, Omran (1971) coined the term epidemiological transition. Two years later, Lerner (1973) presented the 'health transition' as a broader concept. Others also perceived the epidemiological transition as part of the health transition. Mosley (1992) argues that the health transition refers to the demographic transition, the epidemiological transition, the changing risk environment, and the widening health gap across social and economic classes. Frenk et al. (1991) describe the health transition as 'a dynamic process whereby the health and disease patterns of a society evolve in diverse ways as a response to broader demographic, socio-economic, technological, political, cultural and biological changes'. They divide the health transition into the epidemiological transition (changes in health patterns) and the health care transition (the organised response to health conditions).

In response, Omran (1998) argues that it is the other way around: the health transition is part of the epidemiological transition. Epidemiology is the study of health and disease patterns, and their determinants and consequences in various populations. As a result, the epidemiological transition encompasses developments in both dependent and independent variables, and analyses social, economic, demographic, health care, technological and environmental changes as they relate to health outcomes. Classifying all these changes under the 'health transition' would be confusing as health is the dependent variable of epidemiology, not vice versa. Triggered by economic and social development, the epidemiological transition encompasses developments in disease and health patterns (the health transition), fertility and age-structure, lifestyles, health care, (medical) technology, and the environment.

In our view, this debate primarily concerns the use of different terminologies to describe the same process. We will use the term 'epidemiological transition' as a composite concept referring to the long-term changes in health, disease, mortality and fertility patterns and their multiple and interacting determinants. 
The shifts that have taken place in the patterns of mortality and morbidity, particularly in relation to the causes of death and disease, are usually referred to as the epidemiological transition-sometimes also referred to as the health transition (see Box 3.1). It is important to note that the epidemiological changes were paralleled by fundamental changes in health science and its application; Terris $(1983,1985)$ even talks about two epidemiological revolutions: the first against the communicable diseases, and the second against the noncommunicable diseases.

The reasons for our study of the past epidemiological transition are two-fold. First, any exploration of future health should be based on insights from past developments in health. Second, we do not only describe past developments, but also assess what we can learn from the epidemiological transition theory in order to improve our understanding of the broader context of population health. This Chapter first provides some theoretical background regarding the epidemiological transition theory. Subsequently, the epidemiological transitions in the Netherlands and Peru will be assessed. We conclude with a review of the epidemiological transition theory from a systems perspective.

\subsection{THE EPIDEMIOLOGICAL TRANSITION FRAMEWORK}

In 1971, Omran was the first to describe the epidemiological changes experienced in the developed world. He originally described three stages with different mortality patterns and disease levels, building on the demographic transition theory. He updated his theory with a more extended description of the transition and additional historical data in 1983, and added a fourth phase and a futuristic fifth phase (mid-21st century and beyond) to the original theory in 1998. These latter revisions were to a certain extent a response to the fact that in the 1980s other authors already added a fourth phase to his original theory (Olshansky \& Ault, 1986; Rogers \& Hackenberg, 1987). However, these descriptions are complementary and describe more or less the same developments as described by Omran's more recent and widely acknowledged version of the fourth phase.

According to Omran (1998), the epidemiological transition describes 'a longterm shift in patterns of mortality, disease and survival, whereby pandemics of infection and gross malnutrition are progressively, but never entirely, displaced by degenerative, stress and man-made diseases and ageing. These patterns distinguish several major transition stages with overlaps among the stages'. It is important to note that his transition theory is primarily based on changes in the mortality patterns. Additionally, populations can differ in the timing and pace of the epidemiological transition, and that not all follow the same path. This has resulted in the formulation of different transition models, as discussed below. 


\subsubsection{The classical or western transition model}

Omran's (1971, 1983, 1998) classical transition model describes the epidemiological changes in western countries, such as for example Sweden and the United Kingdom. It includes the transition from high mortality (crude death rate of 30-35 per 1,000 population) and high fertility (crude birth rate of 30-35 per 1,000 population) to low mortality (less than 10 per 1,000) and low fertility (less than 15 per 1,000). Life expectancy doubles or even triples to nearly 80-90 years. Pandemics and major epidemics recede, while degenerative stress-related and man-made diseases emerge as an important cause of death. This transition model distinguishes four transition stages.

Stage 1- The age of pestilence and famine

The first stage is characterised by a high and fluctuating mortality- caused by epidemics, famines, and wars-, poor living standards and unsanitary environments. Infectious diseases are responsible for very high mortality, especially among children (infant mortality exceeds 200 deaths per 1,000 live births) and women of childbearing age. Fertility is high, but population growth is low or cyclic. The lack of economic means and knowledge results in insufficient infrastructure for adequate health services, schooling and sewage systems. Health care is provided by systems of herbal remedies, barber surgery, blood letting, bone setting, and so on. Life expectancy varies between 20 and $30+$ years. These conditions prevail throughout most of human history, and continue in Western societies until the late 18th or early 19th century (i.e., until modern times).

\section{Stage 2- The age of receding pandemics}

The second stage is characterised by the control of major outbreaks of infectious diseases and a progressive decline in mortality. Mortality starts to decline around the late 18th century in some countries like England and Sweden. In most other Western European countries and in the United States, mortality decline starts around the mid-19th century. Infant mortality also declines gradually. Important changes in lifestyle, nutrition, hygiene, sanitation and housing take place, public health systems are being built and, later, vaccination programs are being implemented. Infectious diseases are still common and the main cause of death, but the proportion of deaths due to heart disease, stroke and cancer starts to increase steadily. Fertility, however, remains high in the beginning of this stage. As a result of the widening gap between mortality and birth rates (i.e., the demographic gap), population growth is enormous and only partly relieved by the migration to the colonies. Due to high birth rates and improved child survival, the age structure of the population remains very young. About 50 years after the mortality drop, an increase in birth control causes fertility to decline as well. Life expectancy increases to about 50-55 years. 
Stage 3- The age of degenerative, stress, and man-made diseases

This stage is characterised by the continued rise in the proportion of chronic diseases, such as heart disease, cerebrovascular accidents (strokes), cancer, diabetes, chronic obstructive pulmonary disease (COPD), and metabolic disorders. Man-made diseases- caused by radiation injury, chemical and biological warfare, environmental pollutants, motor vehicle accidents, and exposure to carcinogens- and stress-related diseases (e.g., depression) also increases. Although mortality is low, morbidity levels increase due to disease chronicity. This stage becomes more manifest after the First World War (WWI; 1914-1918), but slows down temporarily due the economic crisis in the 1930s and the Second World War (WWII; 1940-1945). Living conditions and sanitation further improve, while modern preventive and curative health care becomes widespread. In this third stage, mortality further declines and eventually reaches stability at a relative low level. Fertility is low as small family size becomes common, women are emancipated and better educated, and marriage as an institution begins to deteriorate. Population growth is low as well. Life expectancy rises further from 50 to $75+$ years, and the ageing of the population sets in.

Stage 4- The age of declining cardiovascular mortality, ageing, lifestyle modifications and resurgent diseases

The distinctive character of the fourth stage is the levelling off and eventual decline of cardiovascular mortality. This unexpected decreasing trend started around 1970 in many developed countries, but some time passed before it was fully recognised through debates in the literature and at conferences during the mid-1970s. Especially the treatment of risk conditions (e.g., hypertension, diabetes, and stress), the influence of medical breakthroughs, and deliberate lifestyle modifications (cessation of smoking, low fat diets, regular and aerobic exercise) are held responsible for this decline. Despite the relative decline in mortality from cardiovascular diseases and some cancers, these diseases remain the leading causes of death. There is a further increase in life expectancy (which approaches 80 to 85 or longer), but also in disease chronicity. Population structure changes due to further ageing. Health care continues to develop, but medical costs for both the state and the individual are very high. Also important are the emergence of new diseases (e.g., HIV, Hepatitis B and C, Lyme Disease) and the re-emergence of old infectious diseases (e.g., tuberculosis, cholera, and malaria).

Besides the above-described classical or western transition model, Omran (1998) also describes the so-called accelerated or semi-western model referring to a transition that is similar to the classical model, but starting several decades later (around 1940) and passing through the different stages much faster. This model describes a shift in epidemiological patterns that occurred most notably in 
Japan, the former Soviet Union, and some Eastern European countries. In addition, this model also encompasses the transition in European populations living outside of Europe, North America and Australia (e.g., Europeans living in Argentina). Most countries in this model, except Japan and Israel, have not entered this fourth stage yet.

\subsubsection{The non-western transition model}

Factors such as poverty, limited education, low status of women, and slow pace of development have been obstacles in the transition in much of the developing world. As a result, the mortality decline was delayed until the mid-20th century. Omran's (1998) non-western transition model describes three stages, of which the first two stages are named the same as in the western model, but differ in timing, pace and underlying processes.

\section{Stage 1- The age of pestilence and famine}

This stage stretches from medieval times to mid-20th century. Conditions such as poverty, lack of food, and primitive health care characterise this phase. Mortality is high and fluctuating, due to epidemics, famine, crop failure and wars. Communicable, maternal, perinatal and nutrition diseases dominate. Examples of prominent infectious diseases in this stage are cholera, plague, malaria, tuberculosis, typhoid, diarrhoeal diseases, hepatitis B, yellow fever and measles, many of which continue to exist at present. Life expectancy at birth is short, varying between 20 and 35 years. Infant mortality exceeds 200-250 deaths per 1,000 live births, while maternal mortality exceeds $1,000-2,000$ per 100,000 live births. Fertility is extremely high, as childbearing starts at a young age and continues until the end of the reproductive span.

Stage 2- The age of receding pandemics

Mortality starts to decline around 1930-1950. The beginning of this stage coincides with the availability of antibiotics, chemotherapy, insect control, immunisation and specific disease control programs. Despite improvements in health care, the access to care and sanitation remains a problem. Fertility is very high, due to the low status of women and low literacy rates, particularly in rural and poor areas. The end of this stage sets in, when fertility finally starts to decline. However, the timing varies between countries, partly due to organised birth control programmes. For some countries, the end of this stage is between the 1960s and 1990s, while others, especially in sub-Saharan Africa, are still in this second transition stage. During this second stage, the demographic gap results in a population explosion and a very young age structure. Life expectancy at birth is between 30 and 45 years old in this stage. 
Stage 3-The age of triple health burden in non-western societies

During this stage, fertility rates decline, but the absolute number of births is still very high due to the young age structure of the population. This third stage is mainly characterised by three major health problems superimposed upon one another. First, unfinished old health problems remain a major issue, especially in rural areas. These include communicable diseases, reproductive morbidity and nutritional deficiency, but also related problems such as poor sanitation, poverty, low literacy rates, and limited access to safe water. Second, new health problems add to this burden. These include the increase in degenerative diseases like heart disease, stroke, cancer and metabolic disorders, as well as health problems such as stress, accidents and man-made diseases (due to, for example, tobacco consumption, sedentary lifestyles or shifts towards unhealthy diets). Additionally, the emergence of new diseases and resurgence of old diseases also are considered as 'new health problems'. New diseases are HIV/Aids, Ebola, Monkey pox, several hemorrhagic fevers, Lyme disease, new strains of E-coli and cholera. Among the re-emerging diseases are malaria, cholera, tuberculosis, dengue fever, plague and others. Third, there is an illprepared health system and insufficient medical training.

It is important to note that Omran (1998) distinguishes several sub-models within the non-western model in order to account for differences in timing and pace within the developing world. The rapid sub-model refers to developing countries experiencing rapid industrialisation or social development. Although these countries are still in the third stage of the transition, they are showing signs of controlling the triple health burden. Good examples are the rapid developments in Hong Kong, China and Puerto Rico. The intermediate submodel refers to countries with middle or lower-middle levels of income that are facing a considerable overlap between communicable and chronic diseases. In addition, the delay in fertility decline excludes these countries from the rapid model. Good examples are the epidemiological changes in Indonesia, Colombia, Mexico, India, Egypt, and the Dominican Republic. Finally, the slow model represents the changes in the least developed and some less-developed countries in Africa, Asia and Latin America. Mortality starts to decline to moderate levels after the mid-20th century, while fertility levels remain high until close to the last decades of this century. The countries in this model are the least prepared to handle the triple health burden.

Besides these sub-models formulated by Omran, the 'protracted polarised transition model' has been formulated as a variant of the non-Western transition model, in order to account for the processes of epidemiological polarisation. According to Frenk et al. $(1989,1996)$ the duration and timing of the epidemiological transition in a given country reflect an average of diverse transitions within the country, occurring in different social groups and different regions. Those living in rural areas tend to have a slower transition than those 
inhabitants of the urban areas do. This can result in epidemiological polarisation, when the duration and timing of these diverse epidemiological transitions within a country differ significantly due to very unequal distributions in such things as wealth, health risks, and health services. Although the features of this process also take place under the other transition models, in the protracted-polarised model, the epidemiological polarisation goes on for longer periods and might be more severe.

According to Bobadilla et al. (1990), the protracted-polarised model has several main features. First, the period of time in which mortality decline takes place is very short compared with the classical model. Second, the onset of mortality decline does not start before the twentieth century and it reaches low levels of mortality near the end of the century. Third, the infectious diseases are not yet brought fully under control and although major reductions are seen in the mortality caused by those diseases, the incidence rates are still relatively high by the end of the twentieth century. This creates an overlap of stages, since simultaneously an increase in non-communicable diseases is seen. Fourth, the distribution of wealth is very unequal and the coverage for health interventions is also incomplete. Because of these inequalities, the gap in health status among social classes and geographical regions is widening. Last, some of the epidemic diseases that had been controlled or eradicated in the past are re-emerging and therefore producing a 'counter-transition'. This reverse transition is clear in morbidity trends that are not considered in the original description of the epidemiological transition.

\subsection{THE EPIDEMIOLOGICAL TRANSITION IN THE NETHERLANDS}

The Netherlands is situated in Western Europe, between Belgium and Germany, and bordering the North Sea. The country has a population of about 16.6 million people and a total area of $41,526 \mathrm{~km}^{2}$ (CIA, 2007). The Dutch population has experienced great health improvements in the past 150 years. In 1861-1865, life expectancy at birth was as low as 36.5 years for man and 38.2 years for women. In 2000, these numbers had increased to respectively 75.5 and 80.6 (Statistics Netherlands, 2006a) (Figure 3.1). This section first provides a brief overview of earlier publications on the Dutch epidemiological transition. Then, it compares the Dutch situation after 1970 with Omran's (1998) description of the fourth transition stage, the 'age of declining cardiovascular mortality, ageing, lifestyle modifications and resurgent diseases'. 


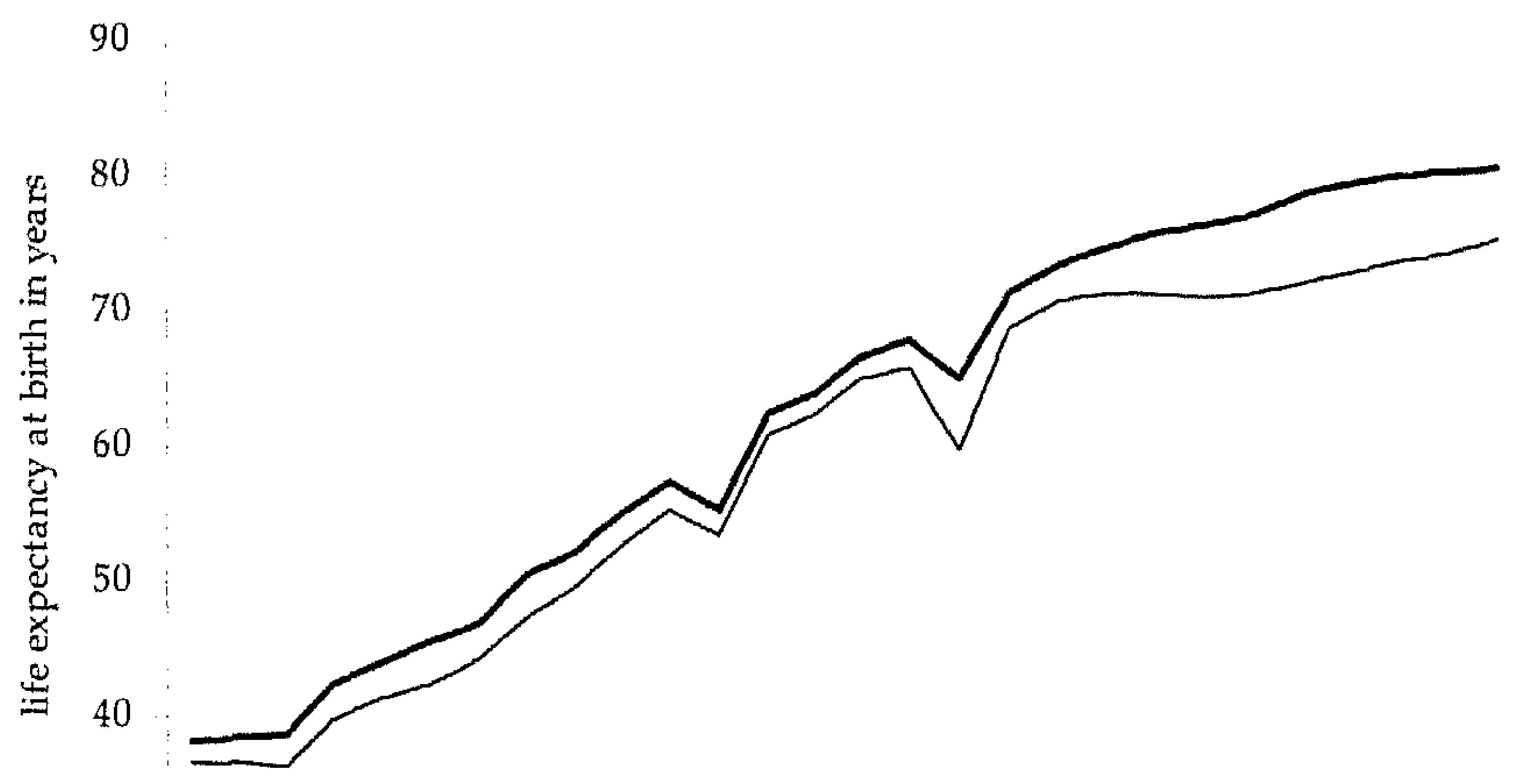

30

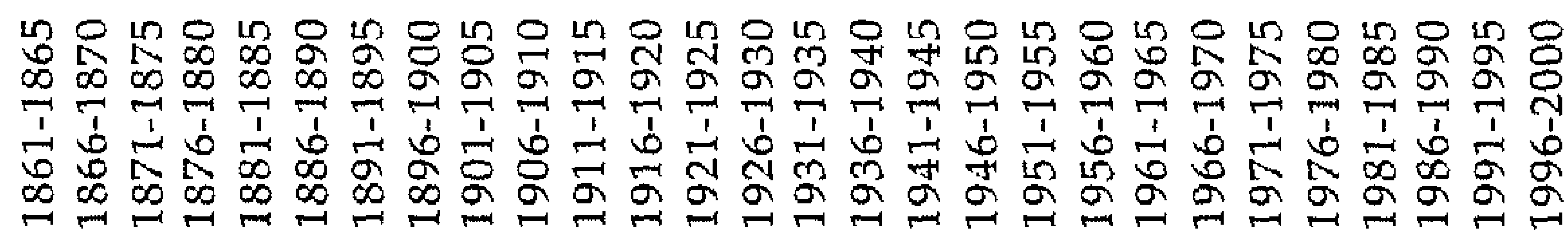

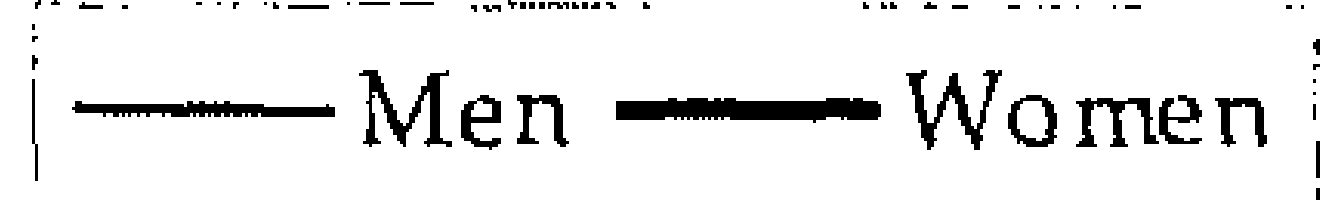

Figure 3.1: Life expectancy in years for men and women, the Netherlands, from 18611865 to 1996-2000 (Statistics Netherlands, 2006c).

\subsubsection{Health developments in the Netherlands: the western model}

There have been several publications (Mackenbach, 1993a, 1993b; Wolleswinkel-van den Bosch, 1998) on the epidemiological transition in the Netherlands, which all focused on the observed mortality decline. In the Netherlands, national registration of mortality did not start before 1811. It is difficult to determine the exact onset of the mortality decline, because the statistics show large fluctuations between 1811 and about 1880 (due to epidemics like cholera and smallpox) (Mackenbach, 1993a). The Dutch cause-ofdeath registration has been a medical one since 1965. From 1975/79, quinquennial mortality data were published, by sex, age and cause-of-death (Wolleswinkel-van den Bosch, 1998). Figure 3.2 shows that in the late nineteenth century, mortality started to decline progressively, followed by a drop in the fertility rate. 


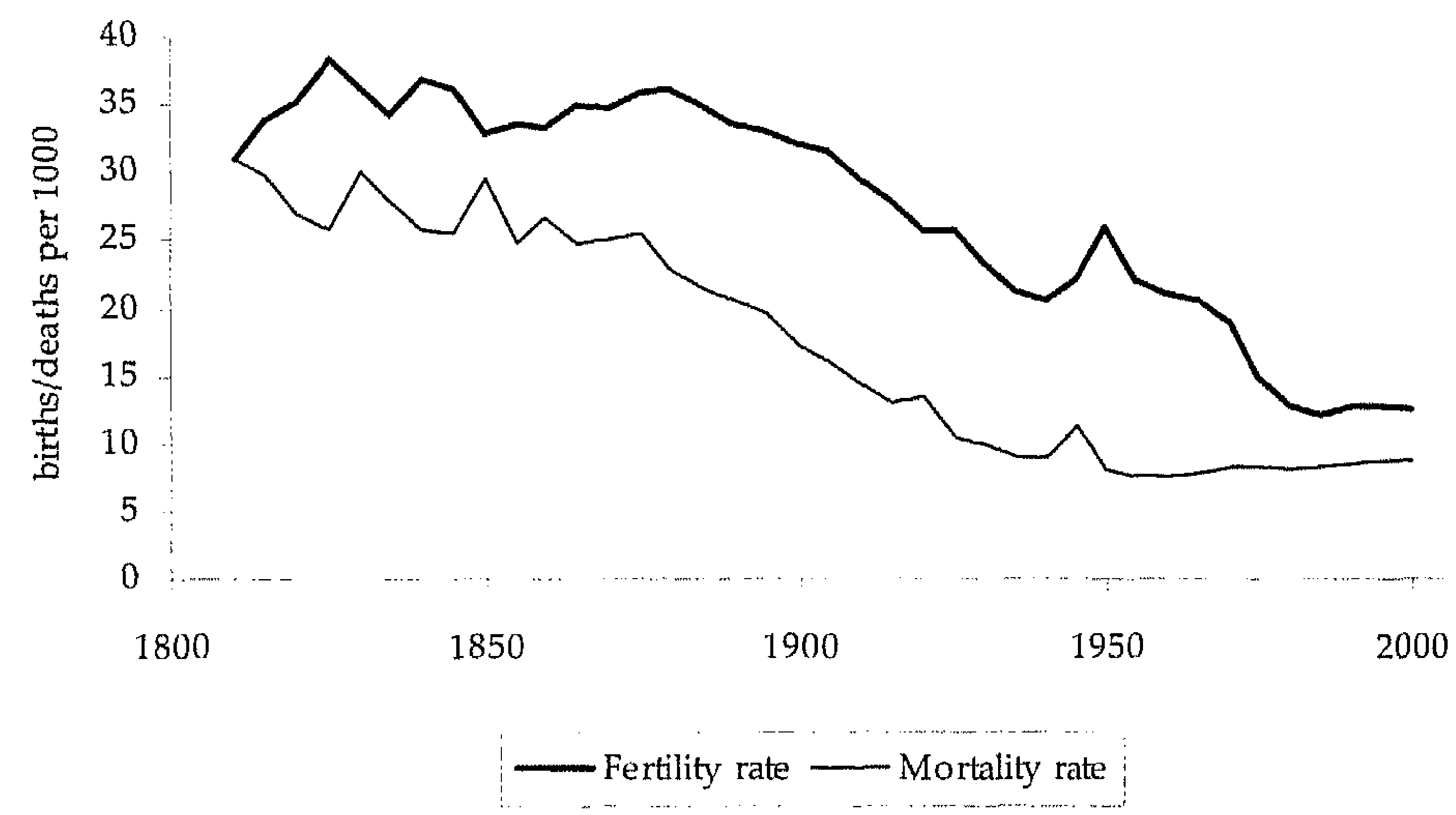

Figure 3.2: Crude mortality and fertility rates, the Netherlands, 1810-2000 (Hofstee, 1981; Statistics Netherlands, 2006a).

Mackenbach et al. (1993a, 1993b) published in the early 1990s on the Dutch epidemiological transition, using reclassified and age-standardised mortality data for 1875/1879, 1903, 1950 and 1970. He concluded that the health developments in the Netherlands were similar to the epidemiological changes in other Western countries, with a strong decrease in overall mortality and infectious disease mortality. Infant mortality dropped by as much as $94 \%$ between 1875-1879 and 1970, whereas in the oldest age-group (80 years and above) mortality declined by $20 \%$ for men and $32 \%$ for women (Mackenbach, 1993b). As a result of declining mortality, life expectancy at birth increased. In 1840-1879, the growth in life expectancy was about one year per decade, but this number increased to about four years per decade in 1879-1920, and to almost seven years per decade in 1920-1930 (Mackenbach, 1993a). In 1875-1879, nearly $40 \%$ of all deaths were attributable to the known infectious diseases like lung tuberculosis, acute diseases of the respiratory tract, and diarrhoeal diseases. Subsequently, mortality from infectious diseases showed a spectacular decrease of $97 \%$. In 1970 , only $4-5 \%$ of total mortality was due to infectious conditions (Mackenbach, 1993b). For many infectious diseases, the main proportion of mortality decline occurred in the period 1903-1930 (Figure 3.3). Mackenbach (1996) concluded that although the largest part of the (infectious) mortality decline occurred before the introduction of modern medical interventions such as antibiotics, the contribution of medical care still contributed to about 4.7$18.5 \%$ of the total mortality decline between 1875-1879 and 1970. In addition, the rate of decline for infectious disease mortality more than doubled after the introduction of antibiotics. 


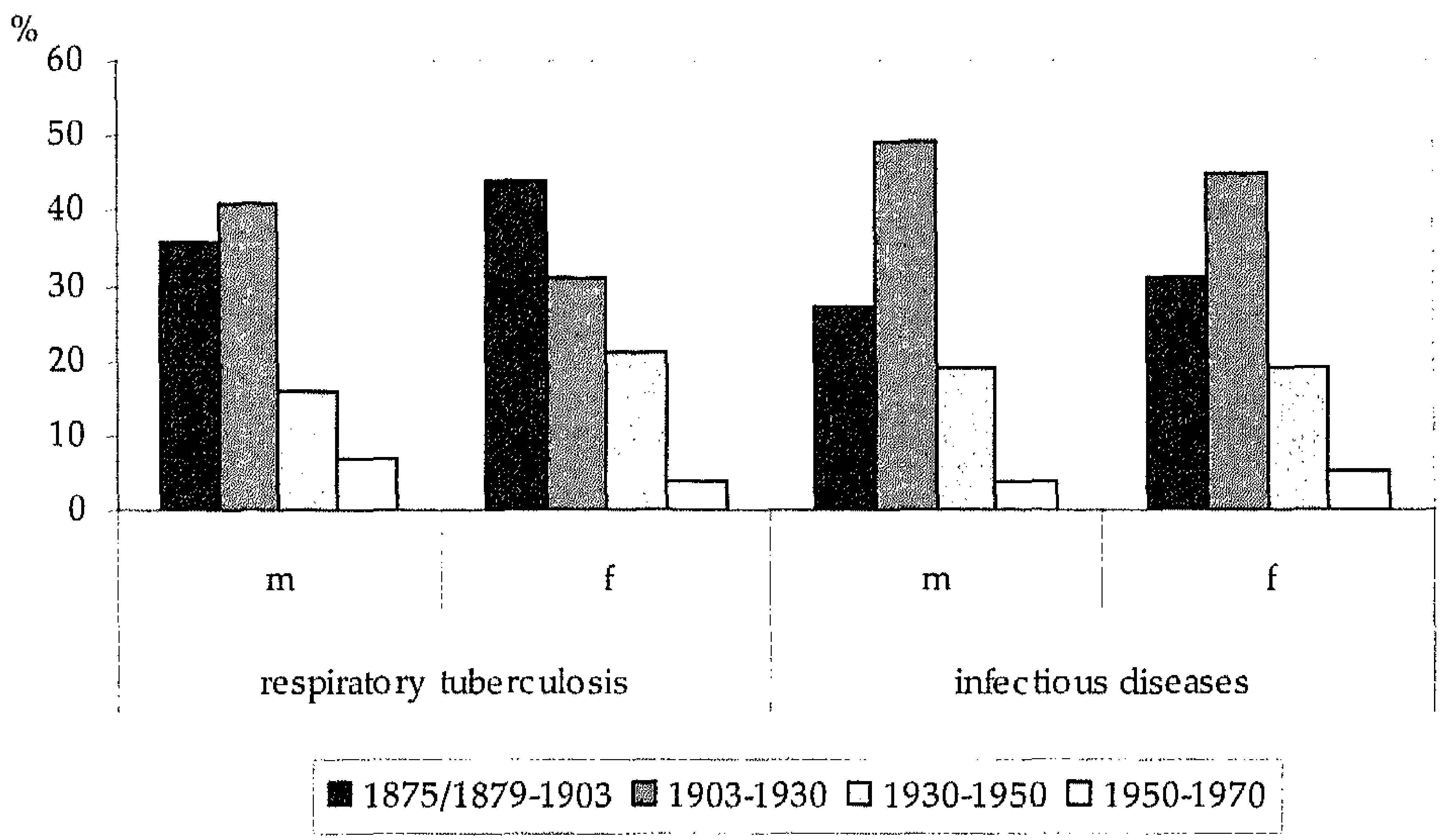

Figure 3.3: The percentage distribution of mortality decline in the Netherlands over four sub-periods, 1875/79-1970 (m=male; f=female) (based on Mackenbach (1993b, 1996).

Mackenbach's (1993b) findings show that the proportion of mortality from cancer and coronary heart disease increased as a result of the strongly declining infectious diseases and the increasing degenerative mortality. The proportion of mortality due to cancer increased from 2-3\% in $1875-1879$ to $25-26 \%$ in 1970 . In the same period, the proportion of cardiovascular deaths increased from $7-8 \%$ to $42 \%$. The group 'other/unknown' causes of death shows a strong decline. Mackenbach (1993b) acknowledges that large parts of this group could actually have been deaths due to infectious diseases or deaths due to degenerative causes. This 'misclassification' could, respectively, result in an underestimation of the decline in infectious disease mortality, or an overestimation of the increases in cancer and cardiovascular mortality.

A few years later, Wolleswinkel and colleagues $(1998,1997,1998)$ published on a more comprehensive study into the Dutch epidemiological transition, using overall-mortality data for the period 1850-1992, and cause-specific mortality for the period 1875-1992 (after reclassifying causes of death). Based on a turning point analysis to determine periods with different paces of mortality change, they concluded that age standardised mortality started to decline around 1880 and that there were several phases of mortality decline in the period 1880-1992. Their main findings are summarised in Table 3.1. 
Table 3.1: Phases of mortality decline in the Netherlands, 1880-1992 (Wolleswinkel-van den Bosch, 1998; Wolleswinkel - Van den Bosch et al., 1998).

\begin{tabular}{|c|c|c|}
\hline $\begin{array}{l}\text { Phase of } \\
\text { mortality } \\
\text { decline } \\
\text { (\% annual } \\
\text { decline) }\end{array}$ & $\begin{array}{l}\text { Causes of death playing a role in } \\
\text { location of turning point }\end{array}$ & $\begin{array}{l}\text { Relationship with determinants of } \\
\text { mortality decline }\end{array}$ \\
\hline $\begin{array}{l}\mathbf{1 8 8 0 - 1 9 1 7} \\
(1.2 \%)\end{array}$ & $\begin{array}{l}\text { Turning point: } 1880 \\
\text { - } \quad \text { other causes (incl, debility) } \\
\text { - } \quad \text { chronic respiratory diseases } \\
\text { - } \quad \text { respiratory tuberculosis } \\
\text { brain diseases, etc. }\end{array}$ & $\begin{array}{l}\text { Improvements in living standards (e.g., } \\
\text { housing, nutrition), working conditions, } \\
\text { socio-cultural change (e.g., fertility } \\
\text { decline) and public health improvements } \\
\text { (e.g., sanitary measures) can all have } \\
\text { contributed to mortality decline in this } \\
\text { period. No large effects of medical factors } \\
\text { expected. }\end{array}$ \\
\hline $\begin{array}{l}1917-1955 \\
(1.6 \%)\end{array}$ & $\begin{array}{l}\text { Turning point: } 1917 \\
\text { - } \quad \text { acute respiratory diseases } \\
\text { - } \quad \text { respiratory tuberculosis } \\
\text { - } \quad \text { acute digestive diseases }\end{array}$ & $\begin{array}{l}\text { Possible determinants of the decline in } \\
\text { this period are further improvements in } \\
\text { living standards (e.g., housing, nutrition), } \\
\text { and medical factors (e.g., tuberculosis } \\
\text { control after WWI, introduction of } \\
\text { antibiotics in 1945). Socio-cultural and } \\
\text { public health factors were of minor } \\
\text { importance. }\end{array}$ \\
\hline $\begin{array}{l}1955-1970 \\
(0.4 \%)\end{array}$ & $\begin{array}{l}\text { Turning point: } 1955 \\
\text { - } \quad \text { hearth disease }\end{array}$ & $\begin{array}{l}\text { The cardiovascular mortality trend was } \\
\text { only slightly increasing due to two } \\
\text { complementary trends: increasing trend of } \\
\text { ischemic hearth disease (due to e.g., } \\
\text { smoking) and a decreasing trend in other } \\
\text { heart disease. }\end{array}$ \\
\hline $\begin{array}{l}1970-1992 \\
(1.1 \%)\end{array}$ & $\begin{array}{l}\text { Turning point: } 1970 \\
\text { - } \quad \text { ischemic heart disease } \\
\text { - } \quad \text { cancer }\end{array}$ & $\begin{array}{l}\text { Important lifestyle changes (smoking } \\
\text { habits) and medical factors (diagnostic } \\
\text { and therapeutic) played an important role } \\
\text { in the renewed mortality decline. } \\
\text { Additionally, mortality shifts to older age } \\
\text { groups. }\end{array}$ \\
\hline
\end{tabular}

In addition, they observed that some infectious diseases started to decline significantly only after WWII (e.g., influenza, syphilis, rheumatic fever), while some non-infectious diseases have already been declining since the beginning of the 20th century (e.g., stomach cancer). Wolleswinkel (1998) concludes that the 'age of pestilence and famine' continued until the later 19th century. The onset of the progressive mortality decline around 1880 indicates the start of the second transition stage, the 'age of receding pandemics'. The 'age of degenerative and manmade diseases' becomes more manifest after WWI, showing a clear increase in ischemic heart disease, cancer and traffic accidents between 1945 and 1970. After 1970, mortality started to decline rather rapidly 
again- as the trend in both cardiovascular and cancer mortality changed from an increasing to a decreasing one, indicating the possible beginning of a new transition stage.

\subsubsection{The fourth transition stage in the Netherlands}

In 1998, Wolleswinkel-van den Bosch concluded that the new epidemiological changes (i.e., renewed mortality decline) observed in the Netherlands after 1970 might be an indication of a fourth transition stage. In the same year, Omran (1998) published his revision of the epidemiological transition, including a comprehensive description of such a fourth stage: the 'age of declining cardiovascular mortality, ageing, lifestyle modifications and resurgent diseases'. To assess whether the Netherlands finds itself in this transition stage, this section evaluates the Dutch situation in the light of Omran's description. Box 3.2 summarises the main characteristics of this fourth transition stage as discussed by Omran (see also section 3.1.1). These will be discussed for the Dutch situation below. This descriptive analysis is based on data from Statline ${ }^{1}$ and the Dutch Association of Comprehensive Cancer Centres (ACCC)2, and additional literature.

Box 3.2: Characteristics of Omran's fourth transition stage: the age of declining cardiovascular mortality, ageing, lifestyle modifications and resurgent diseases (Omran, 1998).

- Further increase in life expectancy

- Levelling off, then decline of cardiovascular and cancer mortality

- Possible causes of the declines in cardiovascular and cancer mortality:

- deliberate lifestyle modifications: cessation of smoking, low fat diets, regular and aerobic exercise

- parallel influence of medical breakthroughs

- treatment of risk conditions like hypertension, diabetes and stress

- Ageing

- Increased disease chronicity

- Resurgence of old diseases and emergence of new diseases

- Improvements in health care systems and mounting medical costs

- Social changes

- Low fertility levels

\footnotetext{
${ }^{1}$ StatLine is the electronic databank of Statistics Netherlands; available from http://statline.cbs.nl.

${ }^{2}$ Available from http://www,ikcnet.nl.
} 
Further increase in life expectancy

According to Omran (1998), life expectancy in the fourth phase is still increasing; this applies in for the Netherlands as well (see also Figure 3.1). Overall, life expectancy for men is lower than for women. In the mid-1970s, this difference in life expectancy grew to nearly six years, but in recent years this gap has been decreasing again. Between 1970 and 2003, life expectancy at birth increased from 70.8 to 76.2 for men, and from 76.5 to 80.9 for women (Statistics Netherlands, 2006a). Between 2003 and 2004, life expectancy increased by as much as half a year to 76.9 year for men and 81.4 years for women. However, this surprisingly strong increase is more likely the result of favourable weather conditions (i.e., less extremes) and the absence of an influenza-epidemic, than an indication of a new trend (de Hollander et al., 2006).

Levelling off and decline of cardiovascular and cancer mortality

The most distinctive characteristic of the fourth transition phase, which also marks its beginning, is the levelling off, and then decline of cardiovascular mortality which occurred around 1970 in many developed countries (Omran, 1998), also in the Netherlands. In 1970-1980, 50\% of all deaths were attributable to cardiovascular causes; nowadays, this proportion has decreased to one-third (de Hollander et al., 2006; Jager-Geurts et al., 2006). Figure 3.4 shows a clear period of levelling off (with some fluctuations) in the 1970s and subsequently a decline in the crude cardiovascular mortality rate. Age-standardised mortality rates even show an earlier decline, starting in the early 1970s; corrected for demographic changes, the risk of dying from cardiovascular diseases more or less halved from 1970 (van der Meulen, 2005). Reitsma et al. (1999) calculated that between 1975 and 1995, age adjusted cardiovascular mortality declined by an average of $2 \%$ per year. In addition, they estimated that about $60 \%$ of the gained life expectancy in this period can be attributed to reduced cardiovascular mortality. Findings by Wolleswinkel $(1998,1997)$ suggest that the declining cardiovascular mortality is primarily due to a decreasing mortality from ischemic heart disease.

Omran (1998) also describes a decrease in some cancers, but without providing any further specification. Figure 3.5 shows that in the Netherlands the crude mortality rate for cancer has been levelling off since the middle of the 1980s. Age-adjusted mortality rates also show a declining trend to about 690 per 100,000 in 2003 (Figure 3.6). It is important to note that this decline has been significantly stronger for men than for women. In 1989-1998, the main increases in age-adjusted mortality occurred for pharyngeal cancer in males, lung cancer in females and oesophageal cancer in both sexes. Declining trends were observed for stomach cancer for both sexes and lung cancer for males (Siesling et al., 2003). Figure 3.7 clearly shows that the age-standardised mortality for lung-bronchus-trachea cancer declined among men, while it increased among women. This observation is primarily caused by deviating trends in smoking 
several decades ago, when the percentage of male smokers started to decline significantly, while the percentage of female smokers increased (Hoogeboezem \& Garssen, 2006). In absolute terms, mortality from lung cancer is still highest in men.

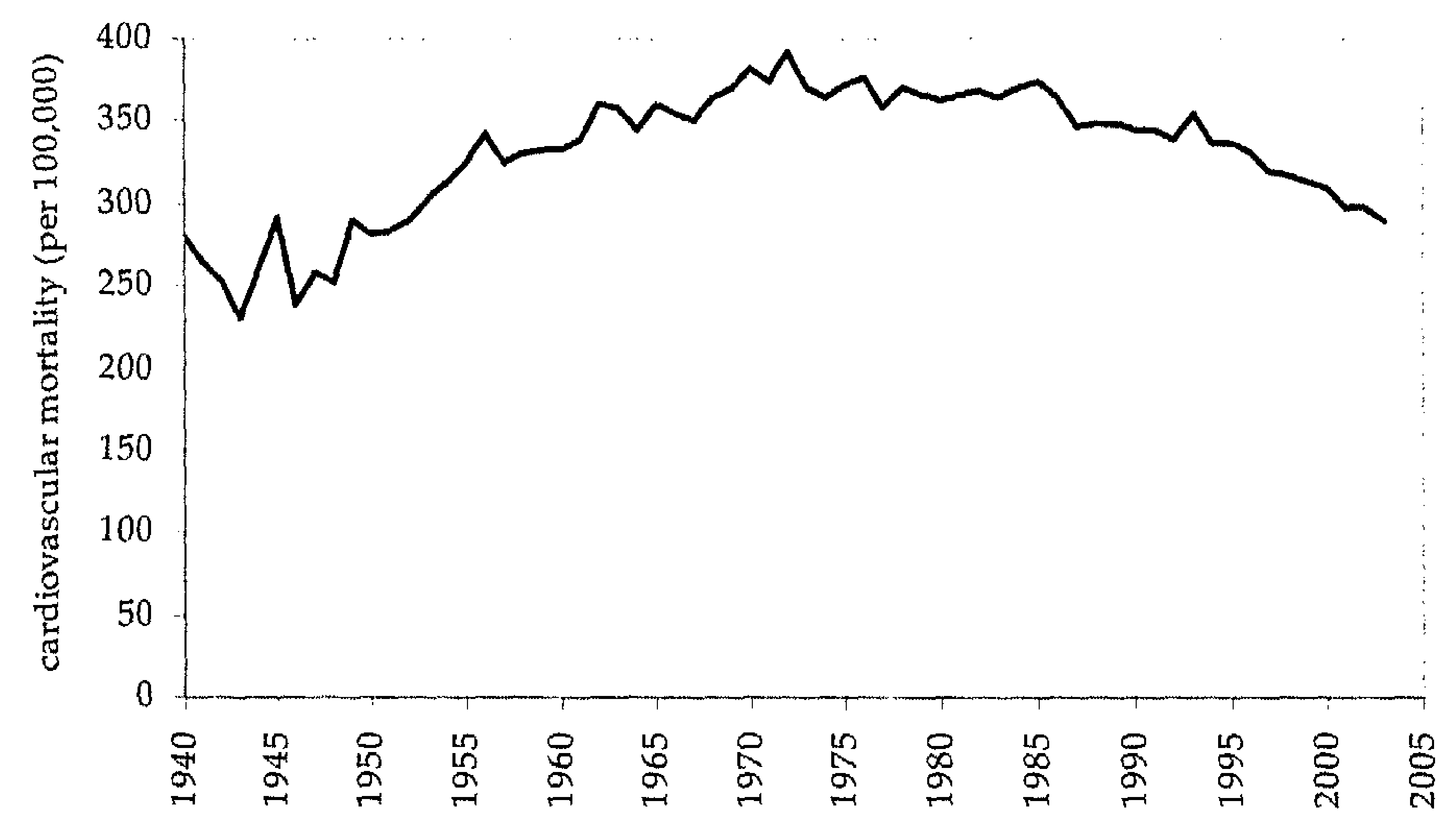

Figure 3.4: Crude mortality rate for cardiovascular diseases (per 100,000), the Netherlands, 1940-2003 (Statistics Netherlands, 2006b).

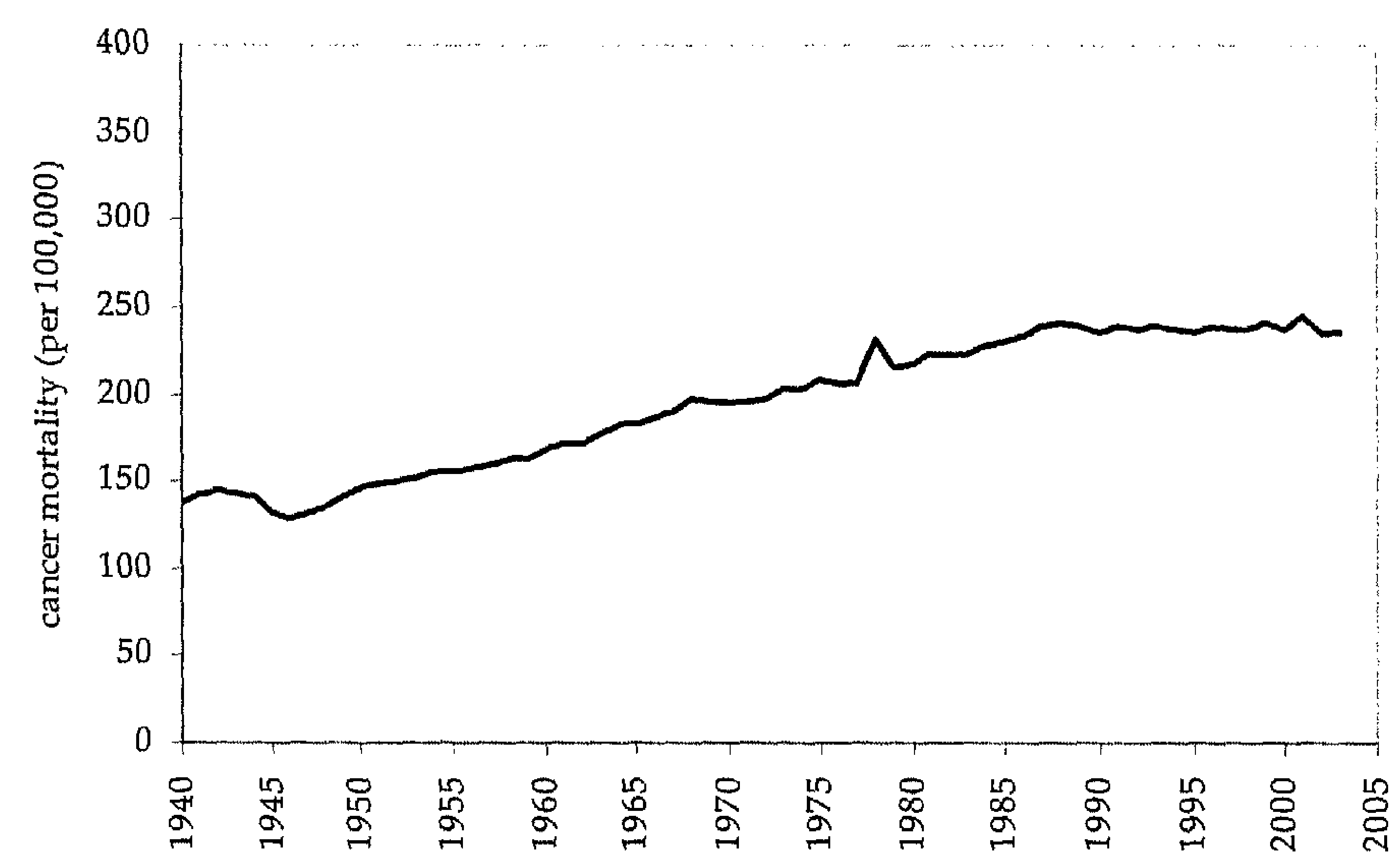

Figure 3.5: Crude mortality rate for cancer (per 100,000), the Netherlands, 1940-2003 (Statistics Netherlands, 2006b). 


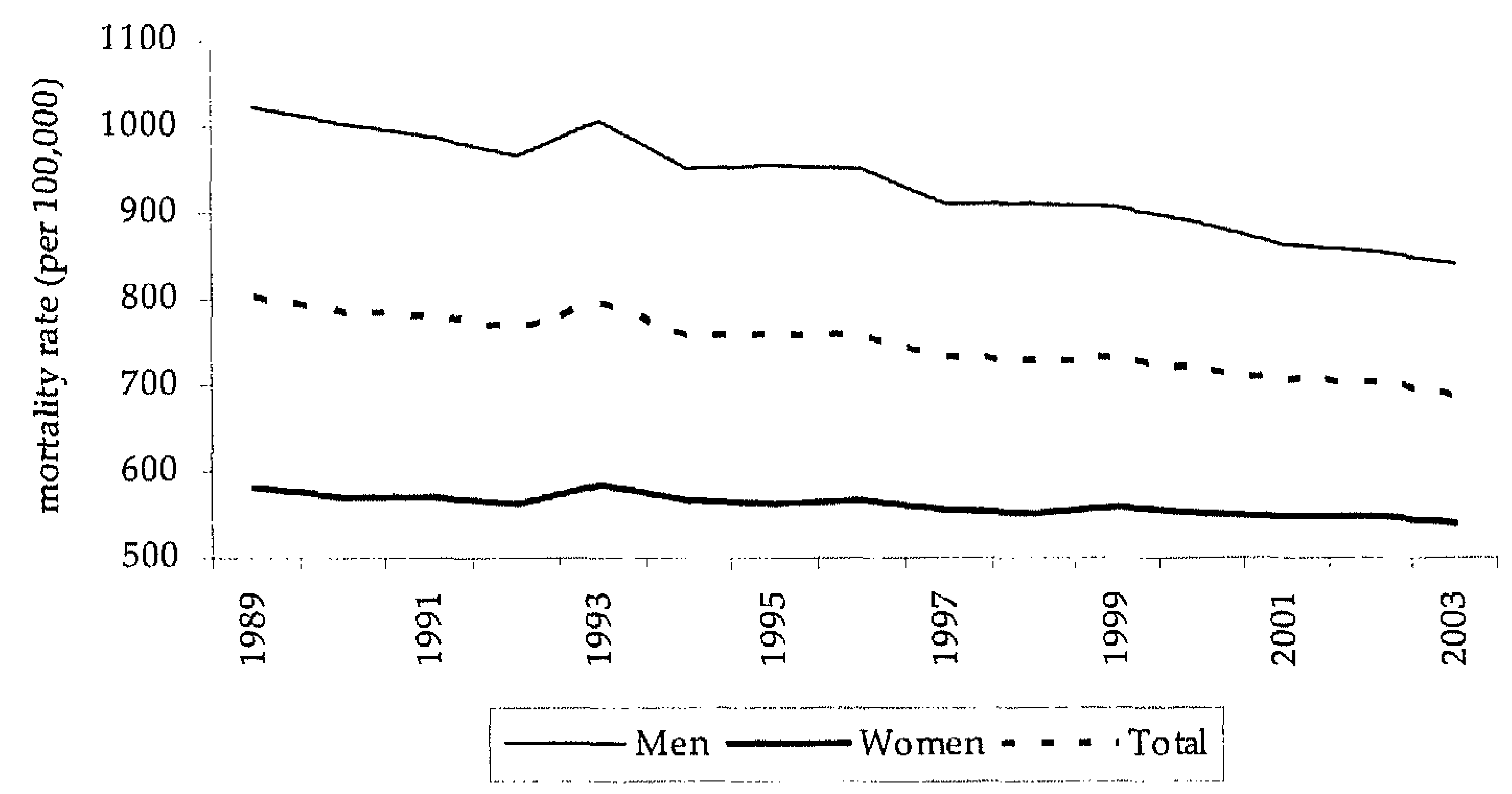

Figure 3.6: Age-adjusted cancer mortality per 100,000, the Netherlands, 1989-2003 (ACCC, 2006).

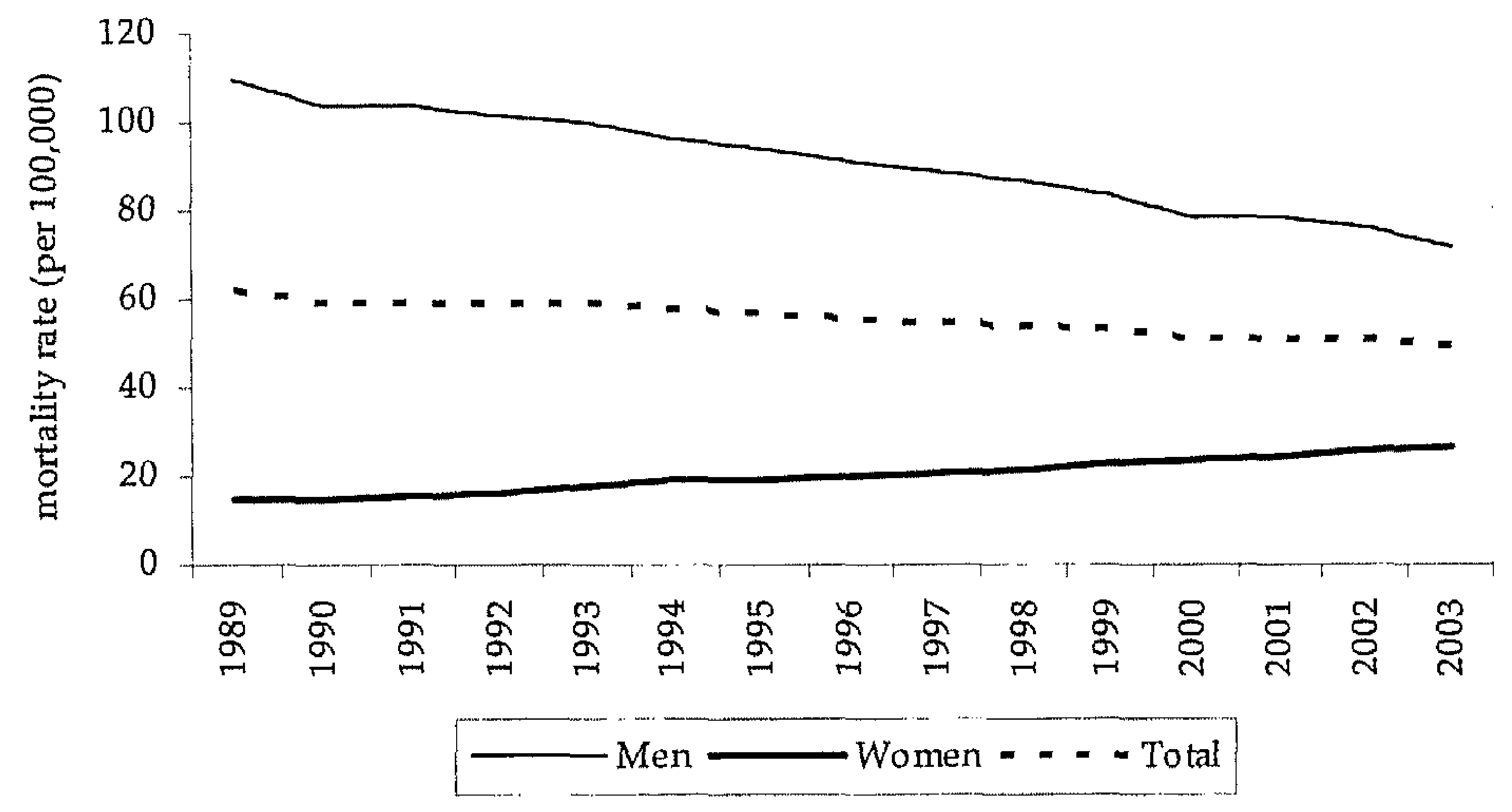

Figure 3.7: Age-adjusted 'Iung, bronchus and trachea'-cancer mortality per 100,000, the Netherlands, 1989-2003 (ACCC, 2006).

The decline in cardiovascular mortality has been stronger than the decline in cancer mortality. Currently, cardiovascular diseases are still the most important cause of death, but it is expected that within a few years most deaths will be attributable to malignant conditions (de Hollander et al., 2006). In 2005, cancer was already the most important cause of death among men (Hoogeboezem \& Garssen, 2006). Between 1970 and 1992, there was also a declining trend in the 
standardised mortality rate for external causes, and more specifically traffic accidents (Wolleswinkel-van den Bosch, 1998; Wolleswinkel - Van den Bosch et al., 1998).

Causes of declines in mortality

Omran (1998) describes three major causes for the declining mortality trends: deliberate lifestyle modifications, medical breakthroughs and the treatment of risk conditions. Regarding lifestyle, it can be concluded that the number of nonsmokers has been rapidly growing from about $40 \%$ in the late 1960 s to about $60 \%$ in 1980 (Stivoro, 2002). In 2004, the number of non-smokers was almost $72 \%$. Among teens (15-19 years), however, the percentage of non-smokers is lower: $55 \%$ for men and $64 \%$ women. It is estimated that about $13 \%$ of the disease burden is attributable to smoking, mainly due to its effects on cardiovascular and respiratory diseases (de Hollander et al., 2006). The decreasing gap in life expectancy between men and women can partly be explained by deviating trends in tobacco consumption. Between 1980 and 2000, life expectancy for men increased by 4.1 years in this period, but for women by only 1.4 years. This growing life expectancy for men is related to the increasing trend in male non-smokers, while the stagnating trend for women has been attributed to increasing numbers of female smokers in the 1960s (van der Wilk et al., 2001; van Oers, 2002).

Food consumption trends have had both positive and negative effects in terms of nutrition. Table 3.2 shows that consumption of total fat and saturated fatty acids declined between the late 1980s and the late 1990s, while the intake of vegetables and fruit unfortunately decreased in that same period (Gezondheidsraad, 2002). The 2003 National Food Consumption Survey (Hulshof et al., 2004) among young adults (19-30 years) showed that none of the respondents consumed the recommended $200 \mathrm{~g}$ of vegetables daily and that only $10 \%$ of the population consumed the recommended $200 \mathrm{~g}$ of fruit per day. Although the intake of total and saturated fats shows a decreasing trend, almost half the respondents consumed a diet containing more than 35\% energy fat. Only $11 \%$ of the young men and $6 \%$ of the young women had a diet with less than $10 \%$ energy saturated fatty acids.

Between 1987-1988 and 1997-1998, the average energy intake decreased, but this decline was not able to prevent the increasing number of overweight people, because average physical activity decreased as well (Gezondheidsraad, 2002). In 1981 , about $33 \%$ of the Dutch population (aged 20 and above) was overweight (Body Mass Index (BMI) $\geq 25$ ). In 2004, this number increased to more than $46 \%$, but then slightly decreased to almost $45 \%$ in 2005 . In 1981 about $5 \%$ of the population was obese (BMI $\geq 30$ ), but this number increased to more than $9 \%$ in 2000 (Statistics Netherlands, 2006d). It is estimated that about 10\% of the disease burden is attributable to obesity, mainly from its effects on chronic diseases such as diabetes and cardiovascular diseases (de Hollander et al., 2006). 
Table 3.2: Average daily intake of foods and nutrients of persons older than twelve in the Netherlands in 1987-1988, 1992 and 1997-1998, standardised to the Dutch population of 1990, in comparison with the recommended levels (Gezondheidsraad, 2002).

$\begin{array}{lllll}\text { Food } / & \begin{array}{l}\text { Recommended intake } \\ \text { (the Netherlands) }\end{array} & \mathbf{1 9 8 7 - 1 9 8 8} & \mathbf{1 9 9 7 - 1 9 9 8} & \begin{array}{l}\text { Percentage } \\ \text { change }\end{array} \\ \begin{array}{l}\text { Total fat intake }(\% \\ \text { total energy intake) }\end{array} & <35 & 38.5 & 36.0 & -6.5 \% \\ \begin{array}{l}\text { Saturated fatty acids } \\ \text { (\% total energy intake) }\end{array} & <10 & 14.8 & 14.3 & -3.4 \\ \begin{array}{l}\text { Vegetables (gram/day) } \\ \text { Fruit (gram/day) }\end{array} & \begin{array}{l}150-200 \\ 200\end{array} & 141 & 120 & -14.9 \% \\ & \begin{array}{l}\text { (or two pieces a day) } \\ \text { (20) }\end{array} & & 102 & -18.4 \%\end{array}$

Between 1987-1988 and 1997-1998, the average energy intake decreased, but this decline was not able to prevent the increasing number of overweight people, because average physical activity decreased as well (Gezondheidsraad, 2002). In 1981, about 33\% of the Dutch population (aged 20 and above) was overweight (Body Mass Index (BMI) $\geq 25$ ). In 2004, this number increased to more than $46 \%$, but then slightly decreased to almost $45 \%$ in 2005 . In 1981 about $5 \%$ of the population was obese (BMI $\geq 30$ ), but this number increased to more than $9 \%$ in 2000 (Statistics Netherlands, 2006d). It is estimated that about $10 \%$ of the disease burden is attributable to obesity, mainly from its effects on chronic diseases such as diabetes and cardiovascular diseases (de Hollander et al., 2006).

Improved medical treatment and technologies could also have played an important role in the observed mortality decline. Omran (1998) mentions some important developments such as early diagnosis, management of cardiovascular diseases, new drugs, cardiac surgery, intensive care, advanced emergency technology and preventive cardiology. In the last decades, major developments in the treatment of heart disease have taken place, also in the Netherlands. Major breakthroughs from the 1960s like percutaneous angioplasty, heart transplantation, beta-blockers and bypass operations were extensively implemented and improved in the 1970s. In addition, important innovations in the 1980s and 1990s like an implantable cardio defibrillator and a coronary stent prolonged life expectancy for heart patients. Besides these technological developments, cardiovascular treatment developed alongside. Before 1970, treatments were primarily focused on coronary care and bed rest. Nowadays, this passive approach has changed into more active interventions and short-term hospitalisation. The importance of treatment in the first hour of attack is increasingly acknowledged (Klaassen et al., 2004).

Additionally, the treatment of risk conditions like hypertension, diabetes (type II) and stress has evolved over the last 40 years (Omran, 1998). Hypertension 
and diabetes are mainly caused by unhealthy lifestyles such as smoking, high fat-intake and physical inactivity, and can therefore be treated to adjust dietary patterns and exercise. In 1980, statin drugs that contribute to the decline of cholesterol levels in the blood were discovered. High cholesterol levels cause hypertension and blood clotting, which cause, in turn, cardiovascular disease. Stress also causes hypertension and therefore increases the risk of cardiovascular disease. Consequently, treatment programmes after hospitalisation for cardiovascular disease nowadays primarily focus on changes in lifestyle (Klaassen et al., 2004).

\section{Ageing}

Omran (1998) also described further ageing as a characteristic of the fourth phase in the health transition. In the Netherlands, the average age of the population increases as more and more people reach old age. Figure 3.8 shows that the proportion of people aged $65+$ was about $6 \%$ between 1900 and 1930 , but increased to $7.7 \%$ in 1950,10.2\% in 1970, 12.8 in 1990 and 14.2 in 2005 (UN, $2007 \mathrm{~b}$ ). The percentage of population aged $0-14$ years declined from $29.3 \%$ in $1950,27.3 \%$ in 1970 and $18.4 \%$ in 2005 (UN, 2007b).

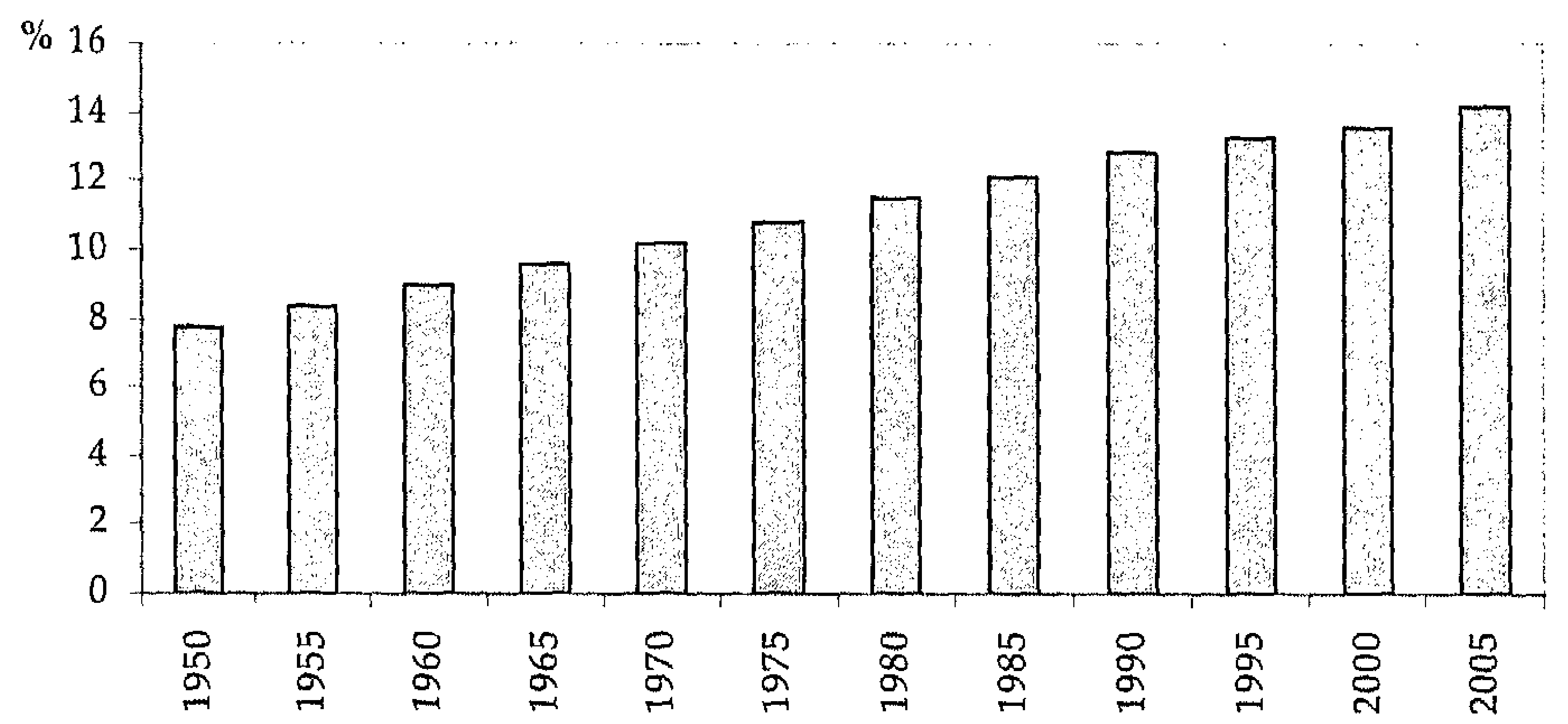

Figure 3.8: Proportion of population aged 65 and over, the Netherlands, 1950-2000 (UN, 2007b).

\section{Increased disease chronicity}

The prevalence of several chronic conditions increased between 1993 and 2003, such as asthma, diabetes, depression, anxiety disorders and stroke. The number of diabetes patients is now about 600,000, partly due to the increasing prevalence of obesity. The prevalence of other conditions decreased, such as gastric ulcers, deafness and heart failure (de Hollander et al., 2006). 
The number of people dealing with the chronic consequences of cardiovascular disease is larger than ever, partly due to improved treatment and medical technologies (van Leest et al., 2002). Due to the increased survival rate after cardiovascular events (e.g., an heart attack), the past decades showed a continuing increase in the pool of people with chronic heart disease and a high risk for subsequent cardiovascular events (de Hollander et al., 2006; Reitsma \& Dalstra, 1999; van Leest et al., 2002). Reitsma et al. (1999) concluded that in 1975-1995 the age-adjusted hospital case fatality decreased, while the number of hospital admissions showed a striking increase, especially for chronic manifestations. Remarkable, however, is the recent decrease in the incidence and prevalence of heart failure, which is probably due to the decreasing severity of coronary heart disease and improved treatment. Mortality from stroke also declined significantly between 1993 and 2003. However, as incidence remained the same (and even increased slightly for women), the number of people living with the consequences of stroke increased (de Hollander et al., 2006).

Table 3.3: Top ten diseases contributing to the disease burden (in Disability Adjusted Life Years (DALYs)) in the Netherlands, 2003 (de Hollander et al., 2006).

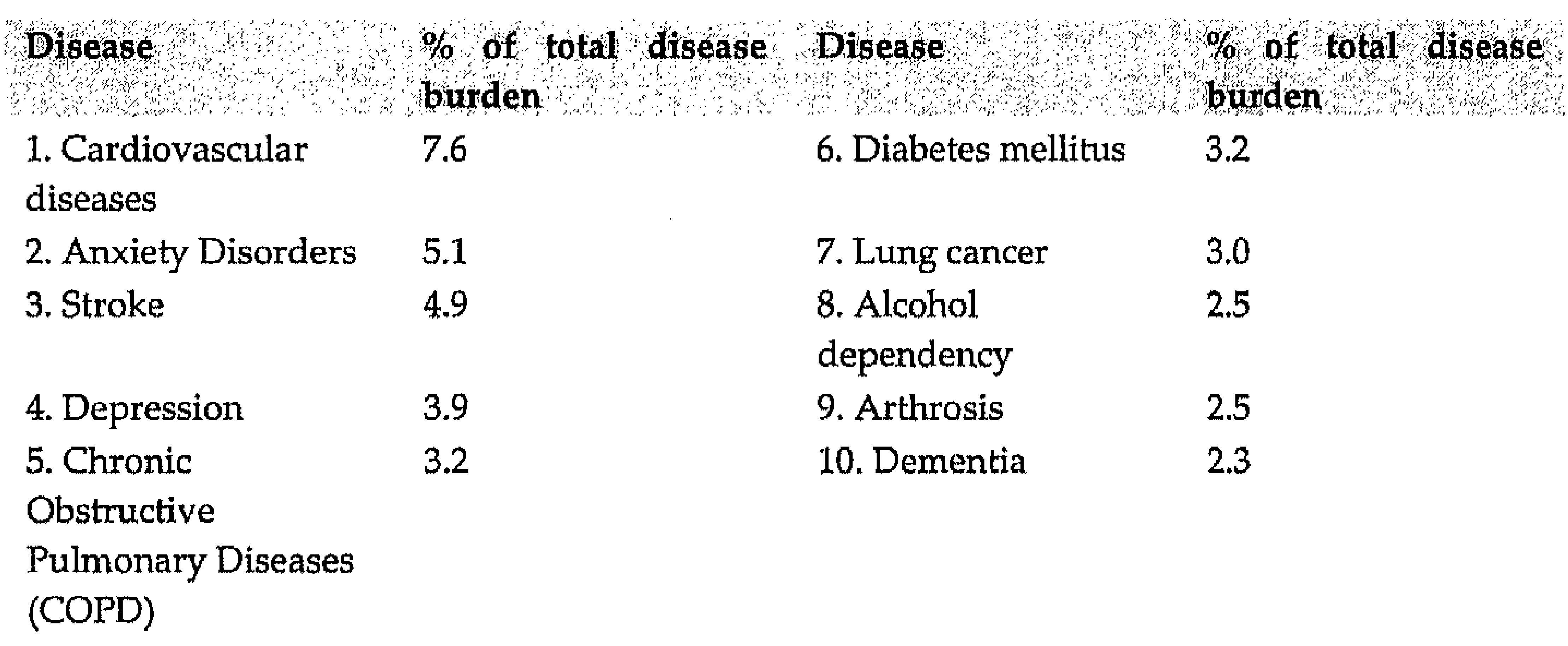

Table 3.3 shows the top ten diseases contributing to the disease burden in the Netherlands. The burden of disease in a population is a measure for the loss of health due to diseases expressed in Disability Adjusted Life Years (DALYs): largely chronic diseases, especially cardiovascular diseases, anxiety disorders and strokes, contribute to the burden of disease in the Netherlands. It is

${ }^{3}$ The Disability Adjusted Life Year (DALY) has emerged as a measure of the burden of disease and it reflects the total amount of healthy life lost, to all causes, whether from premature mortality or from some degree of disability during a period of time. These disabilities can be physical or mental. 
important to note that, even though the prevalence of many diseases is increasing, the number of disabled persons is decreasing due to improved treatments and the use of medical devices (de Hollander et al., 2006).

Resurgence of old diseases and the emergence of new diseases

The Netherlands clearly experiences the emergence of new diseases. An important new infectious disease called Lyme disease is increasingly prevalent in much of Europe, including the Netherlands (Box 3.3) (de Hollander et al., 2006; den Boon \& van Pelt, 2003, 2006).

In addition, HIV/AIDS emerged in the 1980s and the death rate from AIDS, peaked in the mid-1990s (Statistics Netherlands, 2006a). The incidence of hepatitis B increased significantly from the beginning of the 1970s until mid1980s. Subsequently the number of new cases declined again and remained stable at relatively low levels, probably due to changing sexual behaviour and vaccination programmes. However, in recent years the incidence among men is increasing again (Koedijk et al., 2005). Import of infection plays a key role in the Netherlands and sexual contact is the main route of transmission (van de Laar \& op de Coula, 2004). Another important emerging disease is the recent outbreak of Severe Acute Respiratory Syndrome (SARS) in 2003 in the Chinese province of Guangdong. This disease was exported to Western countries like Canada. That the disease has not affected the Netherlands is largely attributed to luck (Timen, 2003).

Box 3.3: Lyme disease is emerging in the Netherlands.

Lyme disease is a bacterial infectious disease, which is caused by the spirochaete Borrelia burgdorferi and is transmitted by Ixodes ricinus (i.e., the sheep tick). This vector is endemic in the Netherlands (van der Poel et al., 2005). It has an unpredictable clinical course with many symptoms. If treated insufficiently, or too late, it can lead to severe disability and illness. Risk groups are people who spend a lot of time in gardens, parks and woods, thereby increasing their chances of being bitten by an infected tick. In Norway, Lyme disease incidence is highest in children aged 5-9 (Nygard et al., 2005).

Between 1994 and 2005, the estimated number of tick bites in the Netherlands more than doubled from 30,000 to 73,000. The number of patients with a bull's-eye shaped rash on the skin (erythema migrans)- as an early symptom of the disease- even increased by about a factor of almost three (from 39 per 100,000 in 1994 to 103 per 100,000 in 2005), which indicates that the risk of infection is rapidly increasing (den Boon \& van Pelt, 2006). In 2005, 17,000 people were diagnosed with Lyme disease by their family doctor (RIVM, 2006). The increasing popularity of outdoor recreation is believed to be one of the most important causes (den Boon \& van Pelt, 2003) and changes in diagnostic procedures could also have played a role (den Boon \& van Pelt, 2006). However, some argue that in, for example, Sweden the increased density and geographical range of Ixodes ricinus in the $1990 \mathrm{~s}$ can be attributed to the milder climatic conditions (Lindgren et al., 2000). 
A re-emerging 'old' disease in the Netherlands is tuberculosis (TBC). Throughout most of the twentieth century, the number of tuberculosis cases has been declining. However, between 1986 and 1994 the number of notifications began rising again from 1,235 to 1,811 (Figure 3.9). This increase is due to the influence of migration from high prevalence countries (Bwire et al., 2000) and increased transmission in high-risk groups like drug users, homeless people and AIDS/HIV patients (Erkens \& Veen, 2006). In more recent years, the number of new TBC cases decreased again to about 1,400 cases per year; most of these infections originated abroad. In 2004, there was an outbreak of Multi Drug Resistant (MDR) TBC, when an East-European TBC patient infected nine persons, from which two developed respiratory TBC (de Hollander et al., 2006).

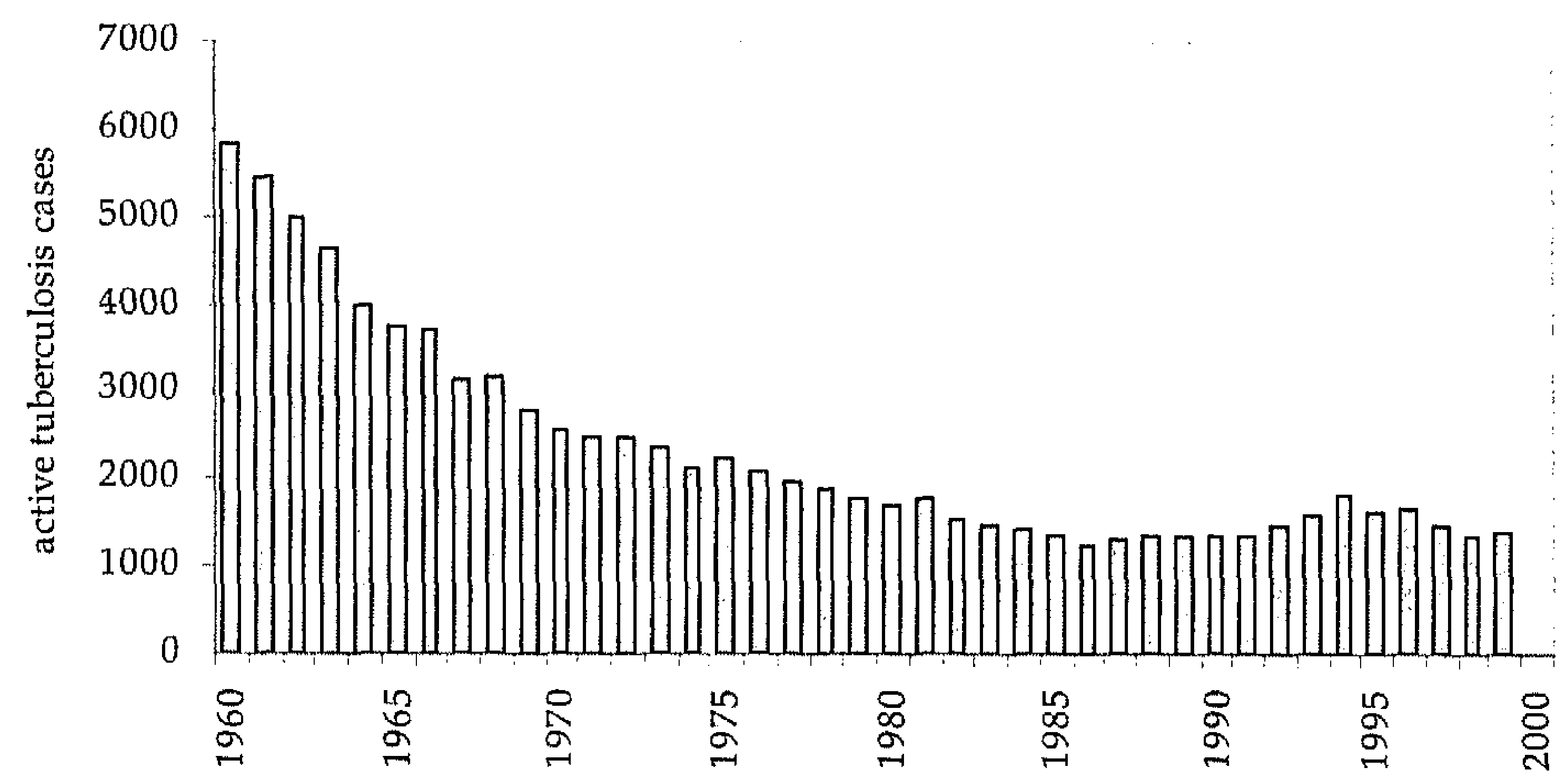

Figure 3.9: Active cases of tuberculosis (TBC), including recidivists, the Netherlands, 1960-1999 (Statistics Netherlands, 2006a).

The number of malaria cases is also increasing in the Netherlands after officially being extinguished in 1970 (Figure 3.10). This increase has been attributed to the increase in tourism to (sub)tropical countries where malaria is common (van der Eerden et al., 2002). 


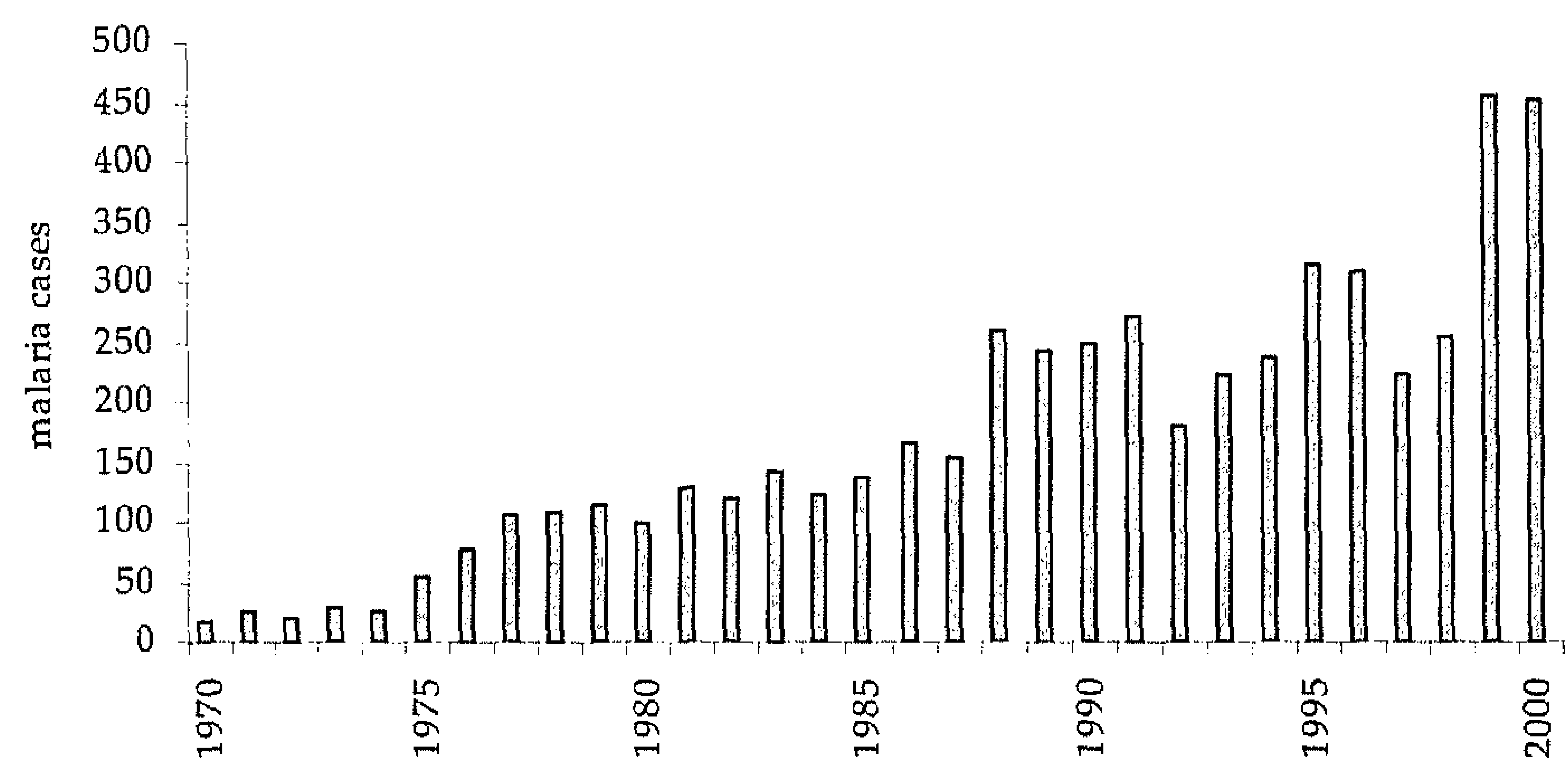

Figure 3.10: Malaria cases, the Netherlands, 1970-2000 (Statistics Netherlands, 2006a).

Improvements in health care systems and mounting medical costs

Omran (1998) mentions that countries in the fourth phase continue to improve their health care systems, including the development of health insurance plans, managed care plans and health care reform, with particular attention to longterm care for the aged and disabled. Intensive medical research flourishes in many areas of health care. The Netherlands changed its health care financing system dramatically in the 1980s, as a result of continuously increasing health expenditures; instead of full coverage health insurance, civilians were increasingly responsible for their individual medical costs. The Dutch insurance company 'Agis Group' surveyed the opinion of the Dutch population about health care accessibility in 1999. A majority of the public believes that everyone in the Netherlands has currently equal access to medical facilities, but expects this will change in the future (Agis Groep, 1999). Data on trends in mortality from selected conditions by socio-economic group show that both higher and lower socio-economic groups have profited from the previous mentioned mortality reductions, probably because of largely equal access to essential health care services with relatively small differences in health care utilisation between socio-economic groups (Mackenbach, 2003). In 2006, the Dutch health insurance system, however, changed again. Instead of the distinction between public and private insurance, there is now a standard health care insurance with optional packages for services such as dental care. Adequate information on the effects of this change is not yet available.

According to Omran (1998), increasing life expectancy and a large burden of disease causes a substantial rise in medical expenditures. Figure 3.11 shows the developments in health expenditure between the early 1950s and 1998. In the 
period 1999-2003, the annual increase in health care costs was as high as $10 \%$, resulting in a total health expenditure of 57.5 billion Euros in 2003. More than half of this increase was due to increasing price levels and $4 \%$ was the result of the increasing volume of care, of which $1 \%$ was caused by demographic developments. About $27 \%$ of all expenses went to hospitals and $21 \%$ to care for the elderly (de Hollander et al., 2006). Health expenditure as a percentage of the Dutch GDP equalled 10.5\% in 2001 and 12.3\% in 2004 (Statistics Netherlands, 2006d).

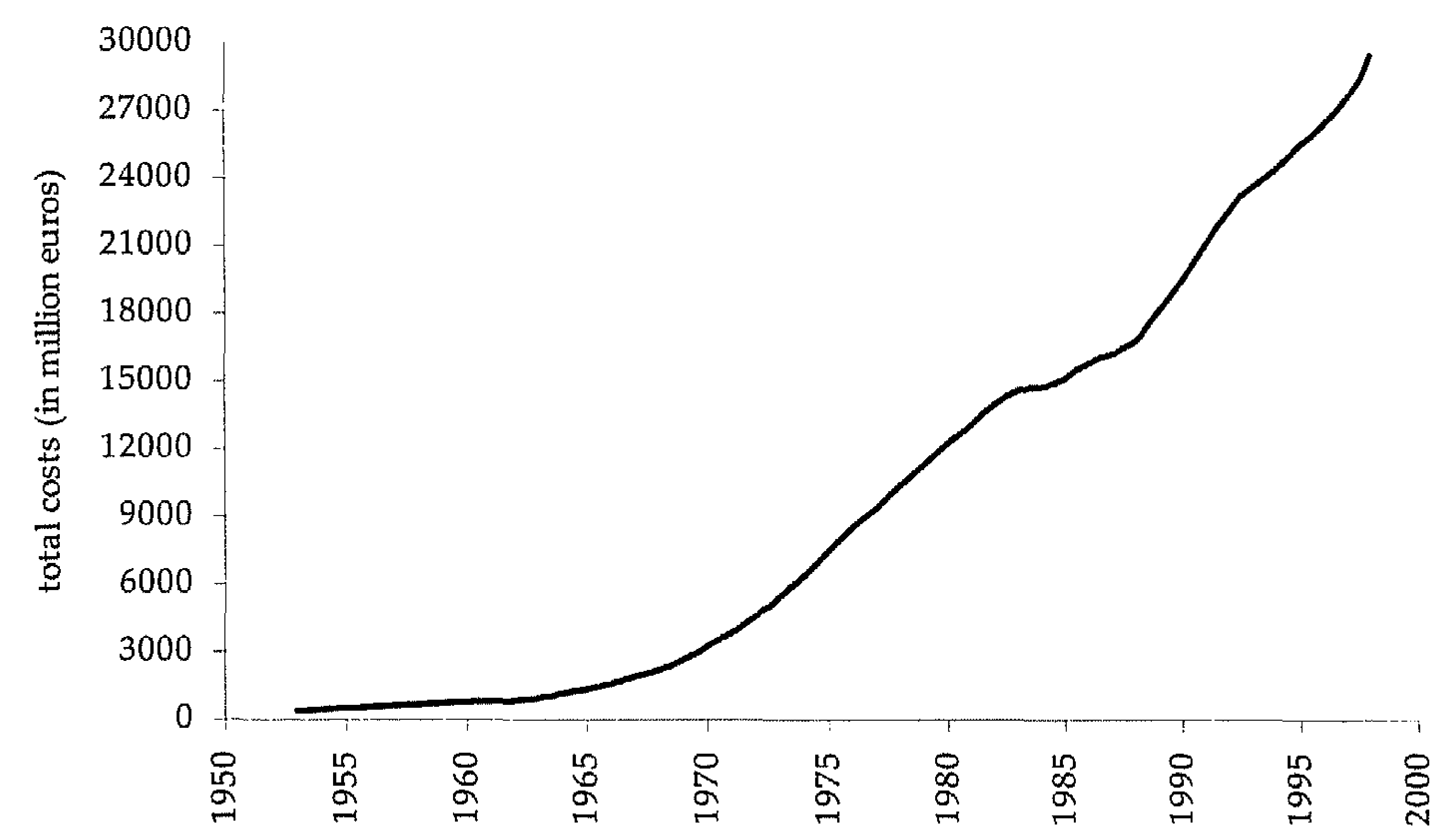

Figure 3.11: Total health care costs in the Netherlands, 1953-1998 (Statistics Netherlands, 2006a).

Social changes

In the Netherlands, household size has declined drastically as fewer people are living in large (married-based) households. The number of single households grew significantly from 387,000 in 1960 to $1,085,000$ in 1980 and 2,449,000 in 2005. In addition, more couples choose to live together without religious or state marriage. Since the 1960s, the women's participation in the labour market has grown. In the early 1980s, about one-third of the women participated in the labour market for more than 12 hours a week. By 2003, this proportion increased to $55 \%$ (Statistics Netherlands, 2004).

Low fertility levels

After the beginning of the 20th century, the number of live births per 1,000 is steadily declining, with the exception of peaks after the two World Wars 
(Figure 3.2). In particular, halfway through the 1960s, fertility dropped very fast because of increased birth control and improved participation of women in the labour market. After this period, from 1980, the fertility rate starts stabilising at a relatively low level. Figure 3.12 shows a declining trend between 1900 and 2004 in the Total Fertility Rate (TFR), which is the average number of children a woman would have if the age-specific rates observed in a particular calendar year were to apply to her entire fertile life span. In the period 1975-2003, the average age of women at the birth of their first child increased from 25 to 29 (Statistics Netherlands, 2004).

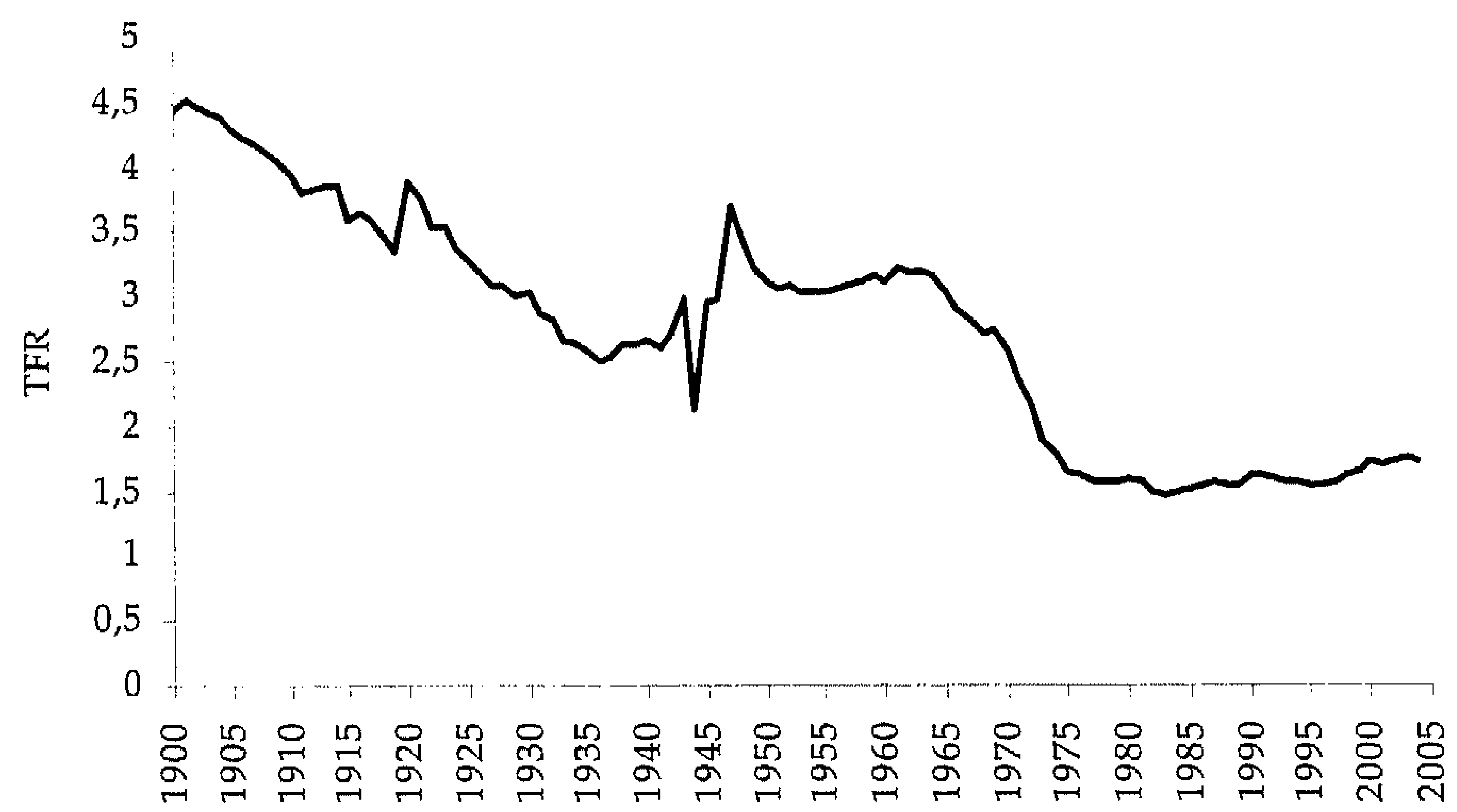

Figure 3.12: Total Fertility Rate (TFR) in the Netherlands, 1900-2004 (Statistics Netherlands, 2006a).

\subsubsection{Discussion}

Previous studies have shown that the past epidemiological changes in the Netherlands are a good example of Omran's western transition model. Trends in life expectancy, mortality and fertility rates reveal health gains. Mortality due to infectious diseases has dropped spectacularly since 1880, and degenerative diseases emerged as the most important cause of death. After 1970, mortality started to decline rather rapidly again, indicating the possible beginning of a new transition stage. However, these studies did not take Omran's description of his fourth stage- the age of declining cardiovascular mortality, ageing, lifestyle modifications and resurgent diseases- into account. Based on the previous section, it can be concluded that Dutch epidemiological changes over the past decades show that the Netherlands currently finds itself in this fourth stage. The most prominent indication for this is the levelling off and, 
subsequent, decline in cardiovascular mortality. In addition, cancer mortality shows a similar trend. As a result, life expectancy increases further and the ageing of the population continues. Omran (1998) suggested several causes for the mortality decline, and most of them could also have contributed to decreasing mortality in the Netherlands. First, there have been some improvements regarding lifestyle. The number of non-smokers increased significantly and the Dutch are consuming diets with lower proportions of (saturated) fats. However, the consumption of fruits and vegetables is declining and obesity is increasing. Both the number of medical breakthroughs and the treatment of people at risk are continuously developing, further improving the prevention and treatment of risk factors and disease. Survival rates for various diseases increased, but- as a result- the prevalence and disease chronicity of many of these diseases increased as well. In addition, the past decades witnessed the (re-)emergence of several infectious diseases. All these developments caused an explosion of health expenditures.

However, developments in health are an ongoing process and do not stop with reaching Omran's fourth transition stage. The epidemiological transition theory provides useful insights on past health developments, but also shows us thatlike many developing countries- the Netherlands still faces some major health challenges. As chronic diseases are more prevalent in the elderly, the ageing process in the Dutch population results in an increasing disease burden and consequent increasing need for health care. The increasing problem of obesity is expected to add to this health problem. Current health problems require a new approach and in the Netherlands the role of the government is currently decreasing. Regarding the prevention of disease, for example, the emphasis is on healthy behaviour and the responsibility of individuals. The most recent Dutch Public Health Status and Forecasts (PHSP) report (de Hollander et al, 2006) stresses the need for an integrated approach, making 'healthy decisions' attractive and feasible.

Additionally, new health risks might arise and effective adaptations to these health challenges have to be developed. The rate of globalisation and global change are unprecedented. Even in the developed world, we need to keep a keen eye on current developments and possible signs of things to come in order to anticipate future health risks and opportunities.

\subsection{The EPIDEMIOLOGICAL TRANSITION IN PERU}

Peru is situated in western South America, between Chile and Ecuador and bordering the Pacific Ocean. Peru has a population of about 28.7 million people and a total area of $1,285,220 \mathrm{~km}^{2}$ (ClA, 2007). Peru has witnessed some great changes in the country's health situation in recent decades. For example, average life expectancy at birth increased from about 44 years in the early 1950s 
to almost 70 years in 2002 (UN, 2003b; WHO, 2004). The country has three very different geographical regions: the coast (Costa), the Andean highlands (Sierra), and the Amazon jungle (Selva). These regions differ from each other with regard to their ecological systems, climate, and socio-economic status. Peru has 25 departments $5^{4}$, which are the country's main administrative units. These departments are categorised into five poverty strata, with stratum I being the richest and stratum $V$ the poorest (Minesterio de Salud, 2001) (Table 3.4). The richest departments are in the coastal region.

Table 3.4: The five poverty strata in Peru (Minesterio de Salud, 2001).

\begin{tabular}{|c|c|}
\hline $\begin{array}{l}\text { Poverty } \\
\text { stratum* }\end{array}$ & Departments \\
\hline I & Tacna, Lima, Callao \\
\hline II & Moquegua, La Libertad, Lambayayeque, Ica, Arequipa \\
\hline III & Ucayali, San Martín, Piura, Junín, Ancash, Tumbes \\
\hline IV & Pasco, Huánuco, Cuzco, Loreto, Cajamarca, Amazonas, Madre de Dios, Puno \\
\hline $\mathrm{V}$ & Huancavelica, Ayacucho, Apurímac \\
\hline
\end{tabular}

This section describes the epidemiological transition in Peru focussing on the observed developments in overall mortality, fertility, population, mortality from infectious diseases, mortality from non-communicable diseases, and the health care system. Accordingly, we will focuses on the diversity in the health situation in Peru, using the concept of epidemiological polarisation. For this descriptive study, six key Peruvian experts 5 were interviewed and an extensive literature and data search was conducted in order to obtain relevant information on the past and present health situation in Peru. With respect to the availability of both historical health data and current health data, it is important to note that Peru has had incomplete registration of health information. For example, estimates of mortality before 1950 are difficult to obtain, and current mortality rates are still corrected for underregistration since nearly $50 \%$ of all deaths in Peru go unregistered (PAHO, 2002). However, the Pan American Health Organization (PAHO) concluded in 1962 that the available mortality data still provided sufficient basis for assessing the main problems and trends

\footnotetext{
4 Peru is divided into 25 departments (including the Constitutional Province of E1 Callao), and the departments in turn have a total of 193 provinces and 1,828 districts (PAHO, 2002).

${ }^{5}$ Marcos Cueto (Universidad Peruana Cayetano Heredia), Carlos Eduardo Aramburú (Consorcio de Investigación Económica y Social), Walter Mendoza de Souza (Instituto de Irvestigación Nutricional), Carlos Bardález del Aguila (Universidad Peruana Cayetano Heredia), Patricia Mostajo (Futures Group), and Magdalena Chú Villanueva (Asociación Peruana de Demografia y Población).
} 
in the country's health status (PAHO, 1962), and the information systems and estimates have been improving ever since.

\subsubsection{Health developments in Peru: the non-western model}

Peru has experienced a significant decline in mortality, from 21.6 deaths per 1,000 inhabitants in 1950-1955 to 6.2 deaths per 1,000 inhabitants in 2000-2005 (UN, 2007b) (Figure 3.13). There has also been an enormous decline in the infant mortality rate, from almost 159 per 1,000 live births in 1950-1955 to 30.3 per 1,000 live births in 2000-2005 (UN, 2007b). Maternal mortality has also declined, from 399 women per 100,0000 live births in 1970 (Bardalez del Aguila, 2001) to 165 women per 100,000 live births in 1994-2000, but Peru still has one of the highest figures for the countries of Latin America (Bardalez, 2002). These declines in mortality rates have resulted in a significant increase in lifeexpectancy (Figure 3.14) (UN, 2007b).

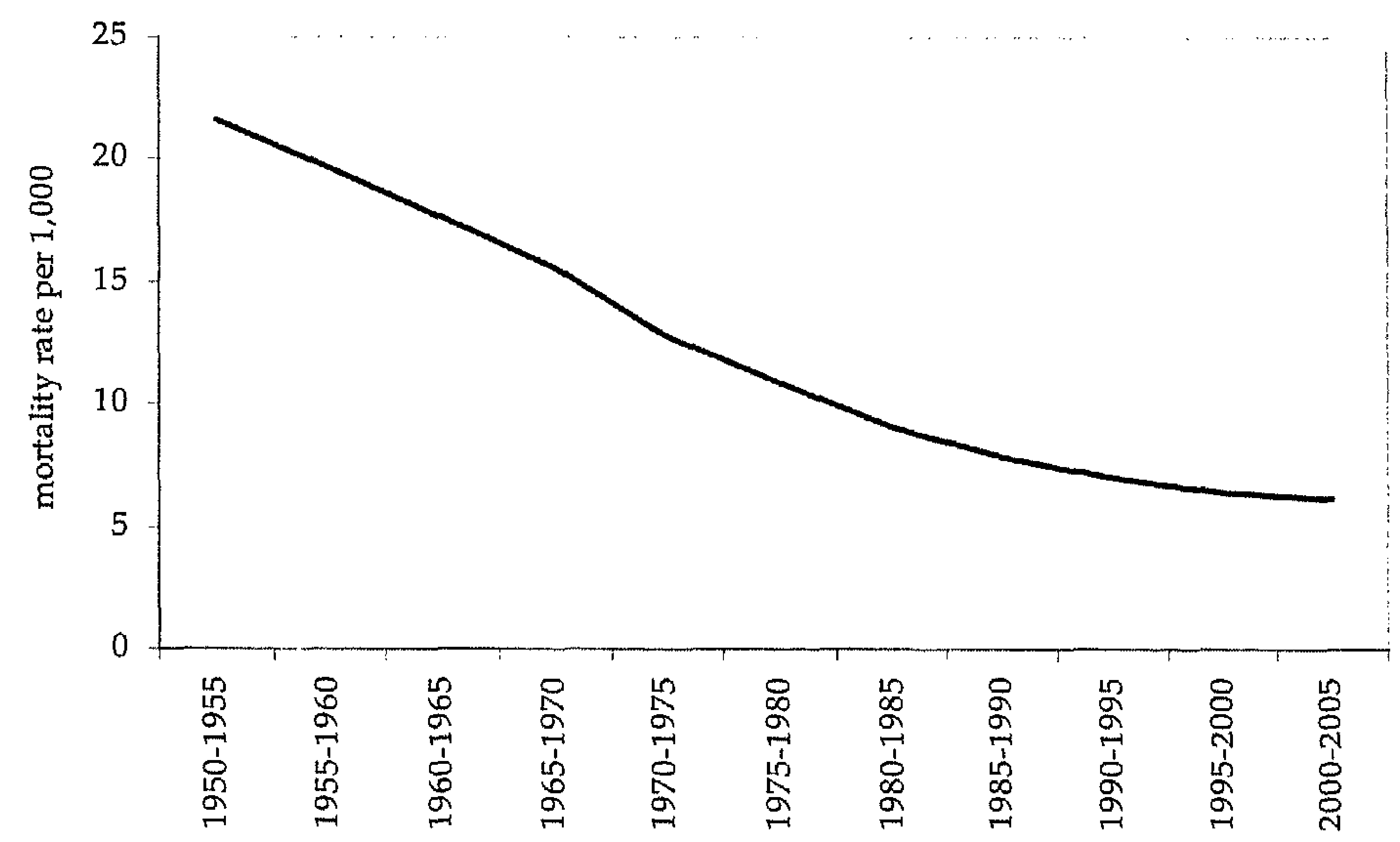

Figure 3.13: Crude mortality rate (per 1,000), Peru, 1950-1955 to 2000-2005 (UN, 2007b). 


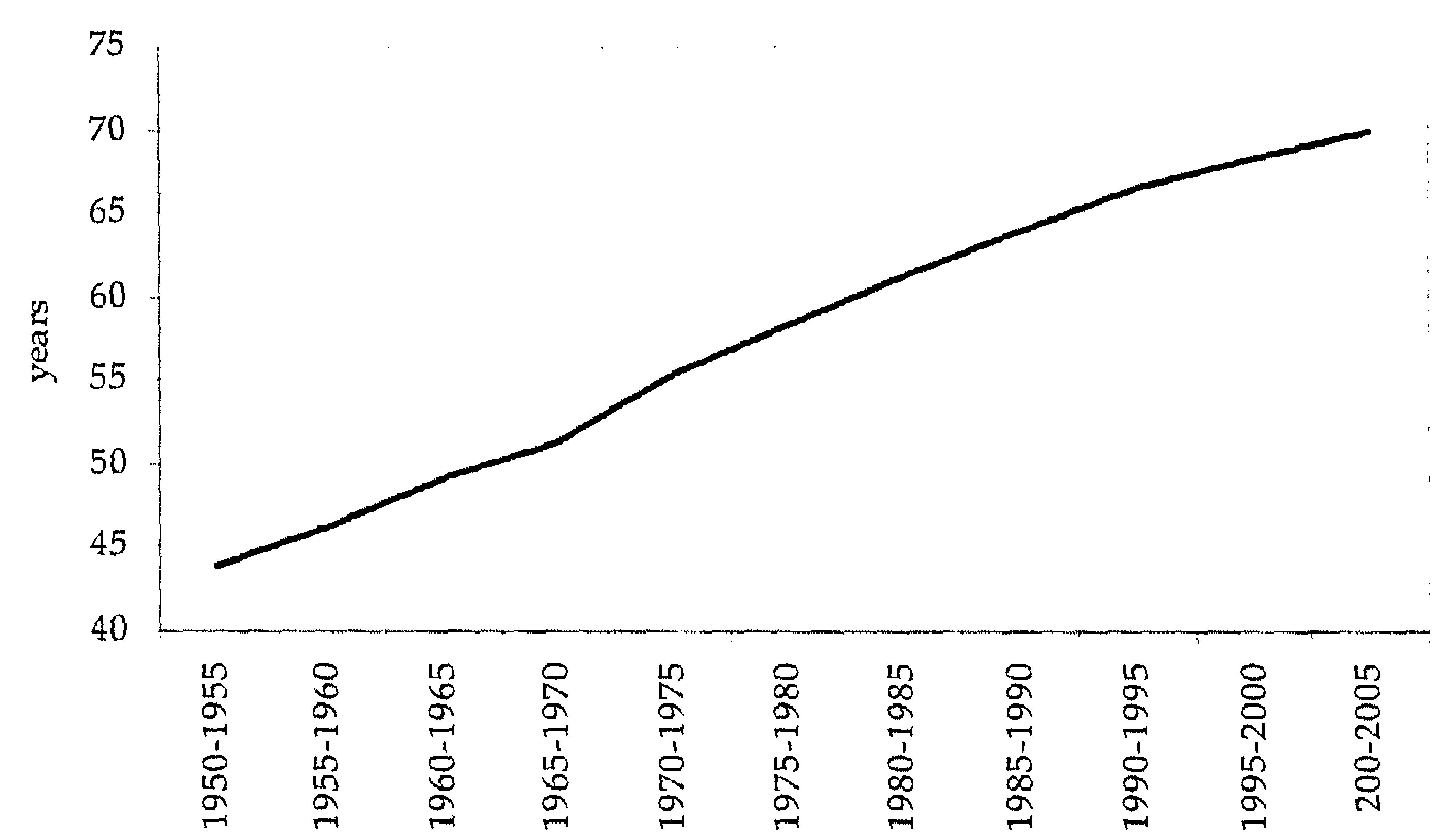

Figure 3.14: Life expectancy at birth, Peru, 1950-1955 to 2000-2005 (UN, 2007b).

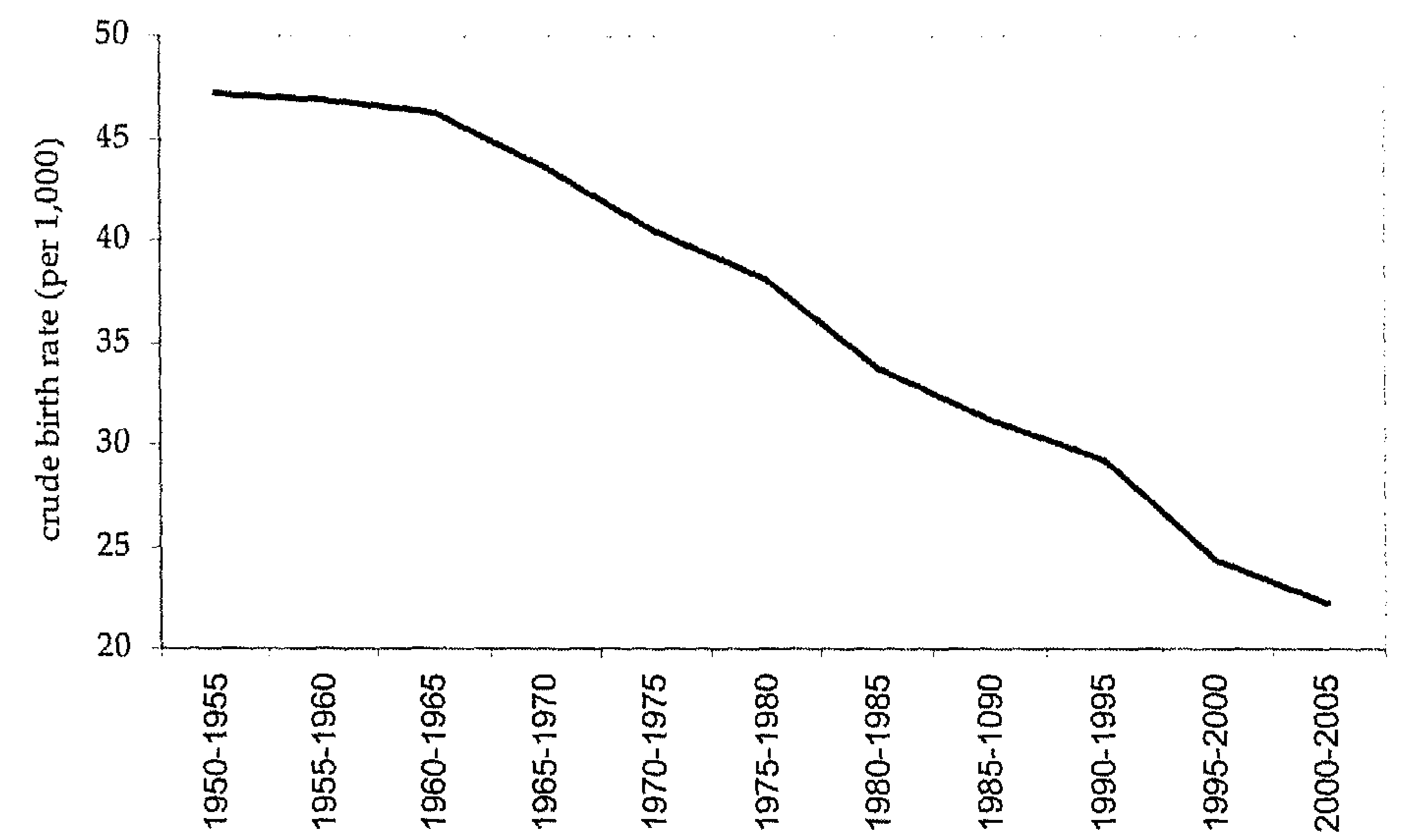

Figure 3.15: Crude birth rate (per 1,000), Peru and Latin America and the Caribbean, 1950-2005 (UN, 2007b)

Another change of great importance in Peru has been the decline in crude birth rate from about 47 per 1,000 in the 1950 s to less than 25 at the turn of the century (Figure 3.15). TFR also showed a significant decline. Between the beginning of 1950 and the end of 1964, the TFR was estimated to be at a high 
level (with it being 6.85 children per woman in 1960-1964). Fertility declined slightly to an average of 6.56 children per woman in 1965-1969. After 1969 the decline accelerated, falling to 4.65 for 1975-1979, 4.10 for 1985-1989, and 3.20 for 1995-1999 (Aramburu, 1995; UN, 2003b).

Because mortality started to decline before fertility did, an increase in the population growth rate occurred. In the 1940s the average population growth was below $2.0 \%$ (PAHO, 1962), but this increased to $2.8 \%$ in the late $1960 \mathrm{~s}$. As expected, this growth rate started to decrease again after the onset of the fertility decline in the early 1970s, falling to an average population growth of $1.7 \%$ in the late $1990 \mathrm{~s}$ (UN, 2003b).

\section{Infectious diseases}

In terms of the causes of death in the period of $1970-1974,44.2 \%$ of all deaths were due to infectious and parasitic diseases (Bardalez del Aguila, 2001) In 1992 , acute respiratory infections still accounted for $16.3 \%$ of all deaths, while intestinal infectious diseases caused $7.7 \%$ of total mortality (PAHO, 1998). There was a decline in mortality from communicable causes from 247.5 deaths per 100,000 in 1987 (PAHO, 2002) to 145.7 deaths per 100,000 in 1996-1998 (Minesterio de Salud, 2001) and 130.3 deaths per 100,000 in the year 2000 (Ministerio de Salud, 2003). Nonetheless, communicable diseases are still the leading cause of death, and acute respiratory infections are the most frequent killers of them all (Ministerio de Salud, 2001; PAHO, 1998, 2002) Among children aged 0-4 years, communicable diseases are the most important cause of death, with respiratory infections and intestinal infectious diseases accounting for $86 \%$ of total mortality (PAHO, 2002).

There has also been a re-emergence of some other communicable diseases in Peru. For example, the overall prevalence of tuberculosis declined in the 1960s and 1970s, to approximately 100 cases per 100,000 inhabitants (Bardalez, 2002), but after 1985 the number of cases increased rapidly, reaching 256.1 cases per 100,000 in 1992 (Bardalez del Aguila, 2001; INEI, 2001; PAHO, 1998). However, the availability of data was very poor before 1990, and it is possible that the large increase observed between 1985 and 1992 is biased by this previous lack of reliable data. After 1992 a downward trend in tuberculosis cases set in, with morbidity rates just below 200 per 100,000 in 1996 (PAHO, 1998) and about 155 per 100,000 in 2000 (INEI, 2001; PAHO, 2002) (Figure 3.16). In the early 1990s, the outbreak of cholera was a major public health emergency. In 1991, 322,562 Peruvians fell sick and 2,909 died of the disease (Cueto, 2001). Fortunately, cholera has been declining ever since, to 934 cases in 2000, with the exception of 1998, when the effects of the El Niño weather phenomenon caused a total of 42,000 cases (PAHO, 2002). 


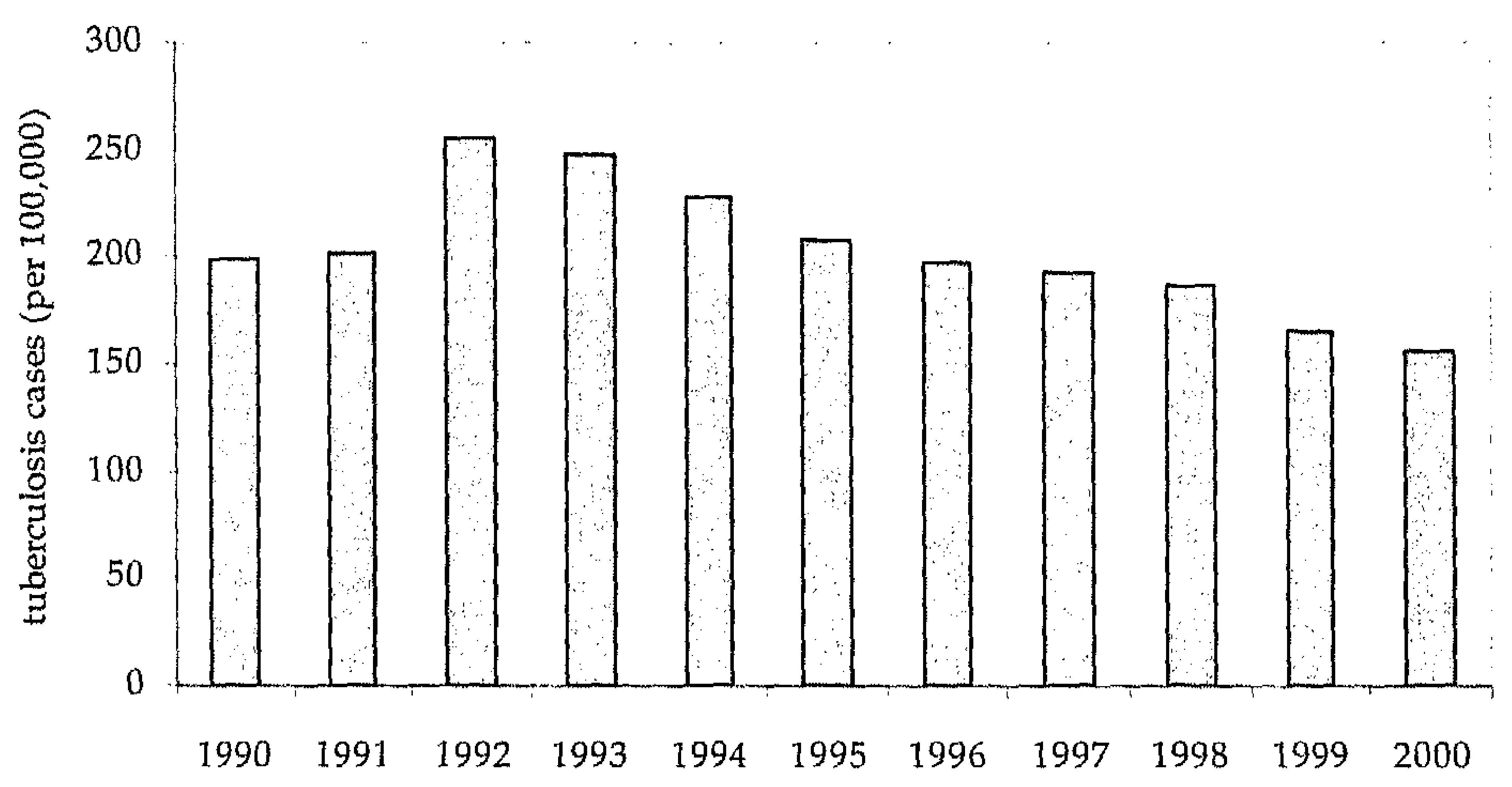

Figure 3.16: Number of tuberculosis cases (per 100,000), Peru, 1987-2000 (INEI, 2001).

Vector-borne diseases are also still major problems for public health in Peru. Three important infectious diseases caused by vector-borne parasites in Peru are dengue fever, yellow fever, and malaria, which are all transmitted by mosquitoes. Although many mosquito control programs were implemented in earlier decades, a re-emergence of these diseases has occurred. For example, the vector responsible for dengue fever was eradicated in 1958 (Bardalez del Aguila, 2001), but the disease re-emerged in 1990, when more than 9,600 cases were reported (PAHO, 1998). In 2000, Peru had an average dengue prevalence rate of 21.4 per 100,000 inhabitants, but some areas showed much higher figures (Ministerio de Salud, 2001).

Peru has experienced a large increase in internal migration and an extensive conversion of jungle land into agricultural and industrial areas. These trends contributed to epidemics of yellow fever, with 503 cases being reported in 1995, with a case fatality rate of $38.8 \%$. Intensification of vaccination activities successfully reduced the incidence to 86 cases, with 34 deaths, in 1996 (PAHO, 1998).

In the past, Peruvian campaigns against malaria were extensive and achieved noticeable results. In the 1960s there were very few cases of malaria (e.g., 16 cases per 100,000 in 1965), but since the 1970s, and especially since 1990, the situation has worsened (Bardalez del Aguila, 2001; INEI, 2000, 2001; PAHO, 1998) (Figure 3.17). A number of factors have contributed to the re-emergence of this disease. These factors include extensive internal migration, the development of new irrigated areas for rice and cotton farming, the spread of the vector, difficulties in managing the control program in hard-to-reach areas, difficulties created by illegal drug activities in the jungle, and the massive 
number of cases in the neighbouring countries of Ecuador and Colombia. In 1996 there were about 885 cases per 100,000 in Peru, and in 1998 an even higher 997 per 100,000 (this latter figure probably due in part to the effects of El Niño) (INEI, 2000, 2001; PAHO, 1998). The re-emergence of the disease resulted in the intensification of malaria control programs (PAHO, 1998), and in 2000 the rate for all types of malaria declined to 266.2 cases per 100,000 (INEI, 2001). The proportion of malaria caused by Plasmodium falciparum, which is responsible for the most lethal kind of malaria, increased from $19.7 \%$ in 1995 to $41.6 \%$ in 1999, but then declined to $30.2 \%$ in 2000 (PAHO, 2002).

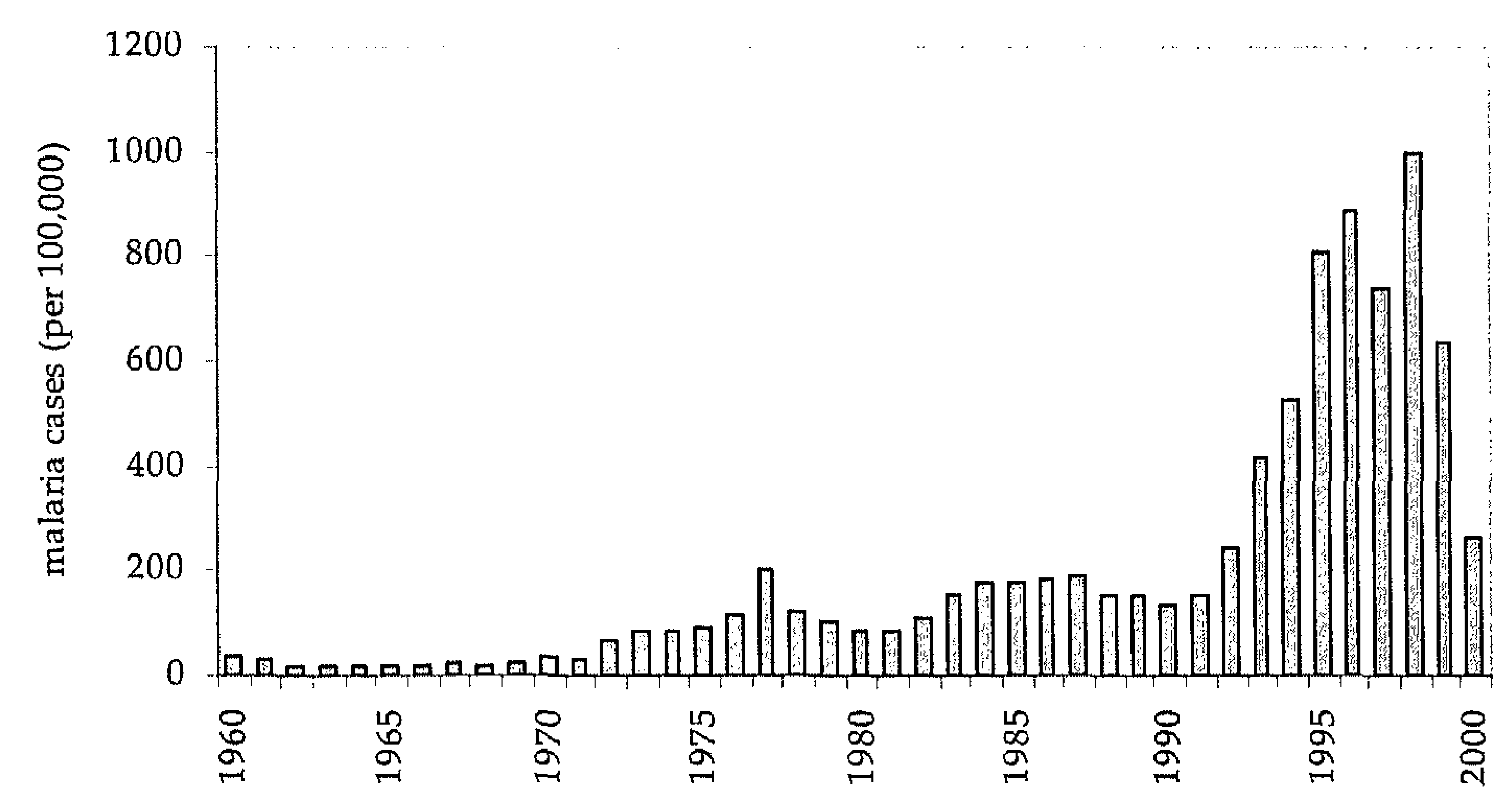

Figure 3.17: Number of malaria cases (per 100,000), Peru, 1960-2000 (INEI, 2001).

Since the first case of AIDS was reported in Peru in 1983 (INEI, 2001; PAHO, 1998), HIV/AIDS has been spreading throughout the country. In 2000 the rate of AIDS in the country was 36.6 cases per 1,000,000, although it is more prevalent in the larger cities, particularly in Lima and Callao (Ministerio de Salud, 2001). The number of persons infected with HIV is many times higher (Bardalez, 2002).

Degenerative diseases

Non-transmissible diseases are an increasingly important problem in public health in Peru, especially in the urban regions of the country (Ministerio de Salud, 1996). Figures for 1994-1998 show that diseases of the circulatory system, cancer, and traumas are together responsible for $40 \%$ of the deaths in Peru (Bardalez del Aguila, 2001). In 1967, diseases of the circulatory system accounted for only $5.4 \%$ of all deaths from defined causes (PAHO, 1970). In 1994-1998, cardiovascular diseases were responsible for $16.3 \%$ of all deaths in 
Peru (Bardalez del Aguila, 2001). In 1996-1998 the mortality rate for cardiovascular diseases was 89.1 per 100,000 (Ministerio de Salud, 2001). Among the elderly, diseases of the circulatory system are the primary cause of death (PAHO, 1998).

In 1972 , only $5.6 \%$ of total mortality was attributable to malignant tumours (Bardalez del Aguila, 2001), but this proportion reached 14.2\% in 1997 (PAHO, 2002). In 1996-1998 the mortality rate for malignant diseases was 93.8 per 100,000 (PAHO, 1998). There are, however, important variances between the different forms of cancer. In females, the frequency of breast cancer has increased, while cervical cancer decreased between 1968 and 1991. In males in the same period, mortality from stomach cancer decreased, but mortality from prostate cancer increased (PAHO, 1998).

Parts of the increases in degenerative health are due to the ageing of the population; the proportion of person aged 60+ increased from 5.6 in 1975 to 7.2 in 2000 (UN, 2003b). The prevalence of unhealthy lifestyles is also increasing in Peru. In 1998, 44.5\% of the population between 12 and 64 years old had consumed tobacco during the preceding year, and $79.6 \%$ had consumed alcohol (PAHO, 2001). Pre-obesity prevalence is very high in most Latin American countries, and $53 \%$ of the Peruvian population has a BMI higher than $25 \mathrm{~kg} / \mathrm{m} 2$ (Filozof et al., 2001). About 34\% of Peruvian women aged 15-49 years are overweight $(25 \leq \mathrm{BMI}<30)$, and $13 \%$ are obese (BMI $\geq 30$ ) (Reyes \& Ochoa, 2001). Smoking, alcohol consumption, and obesity are all related to the development of cardiovascular diseases and malignant diseases.

\section{Health care system}

In Peru, lack of access to health care facilities is an increasing problem, especially for the poor (PAHO, 2001). The percentage of people insured in 1997 ranged from $35.9 \%$ in metropolitan Lima to only $6.2 \%$ in the rural areas. Of the insured people in 1997, 86.5\% was affiliated with the Social Security system, and $6.8 \%$ relied solely on private insurance (PAHO, 2001). In terms of health care facilities, the number of hospitals has fluctuated in recent decades, although these figures can be influenced by the lack of clear criteria to categorise health facilities. There were reportedly 210 hospitals in 1959 (PAHO, 1962), 435 in 1972 (PAHO, 1974), 353 in 1986, and 488 in 2000 (INEI, 2001). In 1959 the number of hospital beds was 2.2 per 1,000 persons (PAHO, 1962). This increased slightly by 1968 , reaching 2.4 per 1,000 population (PAHO, 1970), but fell back to about 1.2 per 1,000 by 1992 (PAHO, 1998). Over that same period there was a concomitant increase in the provision of basic health services and primary care, with the number of health centres and posts doubling in the 1980s (Bardalez del Aguila, 2001). After the 1990 elections, the health sector priorities of the new government were also focused on primary care services. There was a $61 \%$ increase in primary health care facilities between 1992 and 1995, but the number of hospital beds only rose to 1.3 per 1,000 inhabitants (PAHO, 1998). 
The increase in the number of primary care centres in the 1990s was primarily due to the implementation of new health programs, including the Social Expense Targeting Program (Programa de Focalización del Gasto Social) and the Basic Health for All Program (Programa Salud Básica para Todos). In 1997, $31 \%$ of the Ministry of Health budget went to hospitals, and $62 \%$ went to health centres and health posts (PAHO, 1998). In 1999 the public sector provided for $99 \%$ of all health posts, $69 \%$ of all health centres, and $51 \%$ of all hospitals (PAHO, 2001). The number of physicians, professional nurses, and dentists have been increasing, respectively reaching 10.3, 6.7, and 1.1 per 10,000 in 1996 (PAHO, 1998). Unfortunately, medical staff and hospital beds are mostly concentrated in cities and in the most developed regions and departments (PAHO, 1998).

\section{Triple health burden}

The information presented above indicates that the changes in the Peruvian health situation are a good example of the non-Western transition model. The mortality decline in Peru largely took place in the second half of the 20th century, while fertility did not start to decline markedly until after 1970. Therefore, the second phase of this model - the age of receding pandemics-can be placed between the mid-20th century and approximately 1970. Peru is now clearly in the third stage of the non-Western model, with the country experiencing a triple health burden. First, there are unfinished old health problems like 'old-fashioned' infectious diseases (e.g., acute respiratory diseases and intestinal infections) and high maternal mortality rates. Second, new health problems are arising: cardiovascular diseases and malignant neoplasms have become important causes of death, old diseases are re-emerging (e.g., malaria), and new diseases are appearing (e.g., HIV/AIDS). Drug resistance is a problem in the fight against tuberculosis and other diseases. An ill-prepared health system is the third important feature of the age of triple health burden. Peru faces problems regarding the availability of health services (in terms of facilities and medical staff) as well as a low percentage of inhabitants who are insured.

\subsubsection{Epidemiological polarisation in Peru}

Peru is a very diverse country, and its diversity is reflected in its geographical and socio-economic regions. It is, therefore, expected that this diversity is also reflected in the health status within the country. According to Frenk et al. (1989), the duration and timing of the epidemiological transition in a given country reflects an average of diverse transitions within the country, occurring in different social groups and different regions. This can result in epidemiological polarisation, when the duration and timing of these diverse epidemiological transitions within a country differ significantly due to very 
unequal distributions in such things as wealth, health risks, and health services. Table 3.5 shows the differences in health indicators in the Peruvian departments of Lima and Huancavelica. These two departments are chosen to represent the polarisation within the country. Lima (poverty stratum I) is in the coastal region and has a relatively low percentage of poor people, while Huancavelica (poverty stratum $\mathrm{V}$ ) is a rural department in the mountain region and has a relatively high percentage of poor people. There are also great differences between these two departments regarding important health determinants such as chronic malnutrition in children (measured as stunting) and illiteracy rate.

The departments of Lima and Huancavelica differ greatly regarding important health indicators (Table 3.5). In the period 1995-1999, the difference in life expectancy at birth was 20 years: 76.8 years in Lima versus 56.8 years in Huancavelica. The crude mortality rate and the fertility rate are also much higher in Huancavelica (INEI, 2000; Ministerio de Salud, 2003). In 1996-1998, infectious diseases still resulted in more deaths in Huancavelica than did malignant neoplasms and cardiovascular diseases together. In Lima, however, communicable diseases caused less mortality than did malignant and cardiovascular diseases (Ministerio de Salud, 2001). Table 3.5 also shows that infectious diseases were still the most frequent cause of death in Huancavelica in 2000, while in Lima the mortality rate was the highest for malignant diseases (Ministerio de Salud, 2003). Similar patterns could be seen when comparing the causes of death in 1992 in all of the richest, stratum I, departments of the country with the causes in all of the poorest, stratum V departments; in the richest departments, diseases of the circulatory system, malignant neoplasms, and infectious diseases accounted for respectively $22.1 \%, 19.3 \%$, and $21.5 \%$ of all deaths. In the poorest departments, the figures were respectively $10.2 \%$, $4.6 \%$, and $44.0 \%$ (PAHO, 1998).

Health system capacity also differs between Lima and Huancavelica. In 1996, the number of hospital beds per 1,000 inhabitants was 2.4 in Lima and 1.5 in Huancavelica. The difference is even more extreme if one looks at the number of physicians. In 1996, Lima had 18.9 physicians per 100,000 inhabitants, while Huancavelica had only 2.8 physicians per 100,000 inhabitants (Ministerio de Salud, 2001). No information is available about the number of people with health insurance in these two departments. However, in 1997 the percentage of insured people was almost six times higher in metropolitan Lima than in the rural areas of Peru (PAHO, 2001).

Table 3.5 clearly shows that the epidemiological transition has advanced further and that the health care system is more developed in the richer Lima than in the poorer Huancavelica. We could reach similar conclusions by comparing other richer departments of the country with other poorer ones. Due to this wide variety in the health situation and in the health system capacity within the country, the protracted polarised transition model (Bobadilla et al., 1990) (see also section 3.1.2) could be applicable to the epidemiological changes in Peru. 
Table 3.5: Epidemiological polarisation in Peru, as shown by health indicators for the departments of Lima and Huancavelica.

\begin{tabular}{|c|c|c|}
\hline Determinant/Year & Lima & Huancavelica \\
\hline Region & Costa & Sierra \\
\hline Poverty stratum & I (rich) & $V$ (poor) \\
\hline $\begin{array}{l}\text { Poverty (\% of inhabitants) (2002) (Ministerio de } \\
\text { Salud, 2003) }\end{array}$ & 35.8 & 83.7 \\
\hline $\begin{array}{l}\text { Extreme poverty (\% of inhabitants) } \\
\text { (Ministerio de Salud, 2003) }\end{array}$ & 3.9 & 61.6 \\
\hline $\begin{array}{l}\text { Urban population (\% of inhabitants) } \\
\text { (Ministerio de Salud, 2003) }\end{array}$ & 97.3 & 27.0 \\
\hline $\begin{array}{l}\text { Chronic malnutrition (\% of inhabitants aged }<5 \\
\text { years) (2000) (Ministerio de Salud, 2003) }\end{array}$ & 8.3 & 53.4 \\
\hline $\begin{array}{l}\text { Illiteracy rate (\% of inhabitants) (2000) (Ministerio } \\
\text { de Salud, 2003) }\end{array}$ & 3.9 & 17.5 \\
\hline $\begin{array}{l}\text { Life expectancy at birth (years) (1995-2000) (INEI, } \\
2004 \text { ) }\end{array}$ & 76.8 & 56.8 \\
\hline $\begin{array}{l}\text { Crude mortality rate per } 1,000 \text { population (1995- } \\
\text { 2000) (INEI, 2004) }\end{array}$ & 4.1 & 13.0 \\
\hline $\begin{array}{l}\text { Total fertility rate (children per woman) (2000) } \\
\text { (Ministerio de Salud, 2003) }\end{array}$ & 2.1 & 6.1 \\
\hline $\begin{array}{l}\text { Communicable diseases: mortality rate per } \\
\text { 100,000 population (2000) (Ministerio de Salud, } \\
2003 \text { ) }\end{array}$ & 88.6 & 239.6 \\
\hline $\begin{array}{l}\text { Diseases of circulatory system: mortality rate per } \\
100,000 \text { population (2000) (Ministerio de Salud, } \\
2003 \text { ) }\end{array}$ & 79.6 & 165.2 \\
\hline $\begin{array}{l}\text { Malignant neoplasms: mortality rate per } 100,000 \\
\text { population (2000) (Ministerio de Salud, 2003) }\end{array}$ & 93.8 & 123.2 \\
\hline $\begin{array}{l}\text { Physicians per 100,000 population } \\
\text { (Ministerio de Salud, 2001) }\end{array}$ & 18.9 & 2.8 \\
\hline $\begin{array}{l}\text { Number of hospital beds per } 1,000 \text { population } \\
\text { (1996) (Ministerio de Salud, 2001) }\end{array}$ & 2.4 & 1.5 \\
\hline
\end{tabular}

Peru: Protracted polarised transition model

The first feature of the protracted polarised model (see also section 3.1.2) is that the period of time in which mortality decline takes place is very short compared to Western countries. In Peru, the mortality decline took place over a very short period of time in comparison to what happened in Western countries. In the Western world, mortality slowly started to decline around 1750. Mortality began to decline faster in the middle of the 19th century, but it took until the 20th century for it to reach low levels. The rate fell from more than 25 deaths per 1,000 inhabitants in 1860 to less than 10 deaths per 1,000 inhabitants around 1930 (McKeown, 1976a). In Peru, however, the mortality rate was 21.6 per 1,000 inhabitants in 1950, but it was 6.4 in 1995-1999. 
This is also in line with the second feature of the protracted polarised model, which states that mortality does not start to decline before the 20th century and reaches low levels near the end of that century. As already discussed, Peru has experienced a great decline in mortality since the mid-20th century. In the early 1980s, the mortality rate fell below 10 per 1,000 population, even further declining towards the end of the 20th century.

The third feature is that infectious diseases are not yet brought fully under control and that although there are major reductions in the mortality caused by those diseases, the incidence rates were still relatively high at the end of the 20 th century. This creates an overlap of stages, with a simultaneous increase in non-communicable diseases. The overall incidence rate for infectious diseases in Peru is not available, but the mortality rate is still relatively high (130.3 deaths per 100,000 in the year 2000) (Ministerio de Salud, 2003). At the same time, noncommunicable diseases have become an increasingly important public health problem.

The fourth feature of the protracted polarised model is that the distribution of wealth is very unequal and the coverage for health interventions is also incomplete. Because of these inequalities, the gap in health status among social classes and geographical regions is widening. In Peru, higher levels of economic development are found in the highly urbanised coastal regions, and income distribution within the country is very unequal. Between 1997 and 2000, the income gap in Peru became even larger, with the ratio of the income of the richest quintile to that of the poorest quintile increasing from 4.9 to 7.8 . In addition, in the poorer regions there is a shortage of health care services as well as a higher percentage of uninsured persons. This inequality within the country is also reflected in important health indicators such as mortality, fertility, and causes of death. Between 1987 and the late 1990s, there was even a widening of the gaps in mortality from intestinal diseases and infant mortality. Overall, the changes in the specific mortality profile (i.e., the relative importance of the different causes of death) reflect a widening of the mortality gap (PAHO, 2002). The fifth feature is that some of the epidemic diseases that had been controlled or eradicated in the past are re-emerging and therefore producing a 'countertransition.' The re-emergence of malaria and of tuberculosis is an important health problem in Peru (Figures 3.16 and 3.17), as are the recent epidemics of cholera, dengue, and yellow fever.

\subsubsection{Discussion}

Peru has experienced important improvements in its health situation in recent decades, but the health gap between the rich and the poor is still a significant problem. That is, although the population in rural areas has become 'healthier', the urban population is by far the 'healthiest'. 
The Peruvian health situation is a good example of the non-Western transition model. Peru now finds itself in the third stage of this model, as the country is definitely struggling with a triple health burden. Peru has increased its life expectancy in a shorter period of time than did the now-industrialised countries, thus leaving less time for the Peruvian health care system to adjust to the increasing problem of non-communicable diseases. At the same time, the basic health facilities in some areas of Peru are not sufficient to adequately control the still-important, re-emerging infectious diseases. The Ministry of Health has generally focused its resources on surveillance and control of infectious diseases, and it has not yet developed the health infrastructure needed to deal with the increase in non-communicable diseases.

Like many other developing nations, Peru faces a major challenge. The course of infectious diseases and chronic diseases in the country will in part depend on the choices made by the Government, as it considers alternative health development strategies while proceeding through the health transition (Mosley et al., 1992). The epidemiological transition theory provides useful insights on past health developments and current health problems, and this knowledge can be applied in developing an integrated health strategy. A complicating factor for Peru is the wide range and inequity in health status within the country. Any strategy to improve the health situation in Peru must include initiatives to narrow the health gap within the country. These initiatives have to include measures both to increase access to health care services among the poor and to decrease socio-economic inequity overall. This would involve narrowing the gap among different social groups in terms of such important health determinants as income, education, nutrition, and health care. Closing the health gap requires new approaches that more effectively respond to the overall basic needs and interconnected problems that many disadvantaged persons face. Improving the health of all Peruvians will require major investments in public health, an integrated approach towards health by the Peruvian Government, and a strong commitment by the Ministry of Health and policymakers in health-related fields. Noteworthy, our study (Huynen et al., 2005a) was mentioned in a recent editorial article in the 'Boletín epidemiológico' published by the Peruvian Ministry of Health (Bolarte Espinoza, 2006), stressing the need to address the epidemiological polarisation in Peru.

\subsection{THE EPIDEMIOLOGICAL TRANSITION FROM A SYSTEMS PERSPECTIVE}

Since its first publication in 1971, the epidemiological transition theory has been extensively studied, but also debated. One key aspect in these discussions concerned the drivers of the observed changes; several factors have been put 
forward as the most important determinant of change. Others have made more fundamental critiques regarding the rather straightforward definition of the theory and the risk of overgeneralisation. We argue that these debates as well as the case studies provide some valuable lessons about the epidemiological transition theory in view of the more system-based approach adopted in the Ph.D. Thesis.

\subsubsection{The drivers of epidemiological change}

Until the 1960s, it was widely believed that the improvements in our health were primarily due to public health measures and developments in medical care and technology. This perspective was challenge by McKeown (1962, 1972, 1975, 1976a, 1976b), as he argued that improvements in living standards, particularly regarding nutrition, had been most important in determining the mortality decline (with a reduced mortality from respiratory tuberculosis as the most important epidemiological feature) (Box 3.3). Subsequently, he concluded that today's health problems are also more likely to be controlled by environmental improvements rather than medical care.

\section{Box 3.3: McKeown's nutrition thesis.}

In the 1960s and 1970s, McKeown and colleagues published several papers (1962, 1972, 1975, $1976 \mathrm{a}, 1976 \mathrm{~b}, 1988$ ) in which they challenged the long-standing view regarding the importance of medical advances in bringing about the decline in mortality in Britain. He discussed that many infectious diseases had already declined in importance before relevant medical innovations occurred (i.e., before the introduction of sulphonamides and other antibiotics in the 1930s and 1940s). He also concluded that public health measures (e.g., sewage disposal, water supply, milk pasteurisation) were only important from around 1880 . So what has been the main determinant of the mortality decline before this? McKeown argued that about $33 \%$ of the mortality decline in 1850-1900 was due the reduced mortality from water- and food-borne diseases, and about $44 \%$ due to reduced mortality in air-borne diseases. Among the latter, respiratory tuberculosis and pneumonia made the largest contribution. Although large parts of the reduction in water- and food-borne diseases were caused by environmental factors (improved hygiene), these environmental improvements could not have caused any substantial changes in exposure to airborne diseases. Therefore, the mortality decline was primarily caused by an increased capacity to resist (respiratory) infections due to improvements in nutrition.

McKeown's 'nutrition thesis' has been widely discussed over the past decades. As he reasoned by elimination, his critics have often accused him of failing to provide any direct evidence for his 'nutrition' thesis. Although it is widely recognised in the epidemiological community that the lack of adequate nutrition is related to a wide range of infectious diseases, it is still questioned 
whether nutrition has been the most important factor in determining the observed mortality decline. Alternatives that have been suggested include the virulence of infectious organisms, improvements in personal and domestic hygiene, fertility changes, medical intervention, and the sanitary revolution of the second half of the nineteenth century (Harris, 2004). For example:

- Razzell (1974) argued that nutritional improvements could not have been the primary driver of mortality decline, because this decline was not only concentrated in the poor, but also occurred in the rich. He considered smallpox inoculation and, especially, increasing personal hygiene as likely alternative explanations.

- Szretser (1988) stressed that, rather than improvements in living standards and nutritional status, the public health movement (working conditions, housing conditions, education, health services) working through local government should be seen as the main determinant of the 19th-20th century mortality decline.

- Mercer (1990) postulated that decreasing fertility and the consequent reductions in family size could have been important factors in the decline in infectious disease mortality.

- Woods $(1997,2000)$ concluded that the mortality decline was primarily due to a change in virulence of the tubercle bacillus, as respiratory tuberculosis mortality declined at the same rate in all parts of Britain.

- Caldwell emphasised the important role of cultural, social and behavioural change (1993, 1998).

- In 1996, Mackenbach provided three lines of criticism. First, the direct effects of medical care on historical mortality decline are not negligible and perhaps even substantial. Data from the Netherlands, for example, show an acceleration of mortality decline after the introduction of antibiotics. Second, the indirect effects of medical care on historical mortality decline should also be considered. It is possible that, before the introduction of sulphonamides and antibiotics, medical care and medical doctors influenced sanitary reform (e.g., hygienic measures, improvements in housing and working conditions), and important cultural changes (e.g., education system promoting the public good, economic investments, birth control) that preceded the rising living standards. Finally, medical care could be an important factor in controlling today's health problems, as it is an important factor in more recent mortality declines.

- Omran $(1983,1998)$ argued that in the western transition-model, decreasing mortality is more socially than medically determined, with changing socioeconomic factors and modernisation as the primary drivers, augmented by the sanitary revolution in the late nineteenth century and by medical and public health progress in the twentieth century. He discussed that important changes in lifestyle, nutrition, hygiene, sanitation and housing took place first. In addition, governments started to build public health 
systems, and early 20th century vaccination programs were implemented. On the other hand, he claimed that the transitions in non-western societies are primarily medically determined by medical progress, organised health care, and (international) disease control programs.

All of the suggested alternatives to McKeown's nutrition thesis have been subject to critique themselves as well. Even today we cannot say with certainty what proportions of the observed mortality decline and epidemiological change were due to medical advances, improving living standards or lifestyle changes (Grundy, 2005).

\subsubsection{Critiques on the epidemiological transition concept}

Although the epidemiological transition theory is widely considered as a useful framework for the multiple health changes in populations, it is important to note some critical comments made over the past decades. In 1994, Mackenbach commented on the classical transition model, arguing that that the concept of the epidemiological transition is ill-defined and ambiguous. He postulated that Omran's first stage stretches from prehistory into modern times, as mortality has always been high and fluctuating. It would, therefore, be more appropriate to locate the beginning of the transition between the first and second transition stages. Furthermore, he argued that it is difficult to determine the beginning of the transition (due to lack of adequate data), as well as the end of the transition (due to difficulties in determining the specific causes of death to be included in such analyses). Finally, Mackenbach considered the possibility of several subsequent epidemiological transitions rather then stretching Omran's description to include new developments.

Other important critiques deal with the possibility of overgeneralisation. According to Kunitz (1990), 'it is possible to commit the historic fallacy... and assume that development stages are everywhere the same and follow one another in some inevitable progression'. Gaylin and Kates (1997) felt that the assumed generalisability of the epidemiological transition concept may undermine large differences among population subgroups. Frenk, Bobadilla and colleagues (Bobadilla et al., 1990; Frenk et al., 1989) also addressed this issue of epidemiological polarisation in their protracted-polarised transition model.

In 1998, Omran dealt with some of these important comments in his second revision of the epidemiological transition theory. Besides adding a fourth stage, he further elaborated on the additional transition models representing variations in passing through the stages, recognising that countries can differ in the timing and pace of the transition, and that not all countries follow the same path. He also discussed that there are differentials in health and disease changes among different sub-populations within countries. Second, he argued 
that, despite the lack of continuous and reliable health data in pre-modern and pre-industrial countries, an 'assessment of the possible range of variations in mortality, fertility, disease pattern and age structure does allow probabilistic conclusions'. Finally, he clarified that stagnation or reversal of the transition is possible during economic, political, environmental, morbidity or other crises (i.e., counter-transition). On the other hand, the transition may accelerate under favourable conditions.

In 1998, Wolleswinkel argued that the conventional view of a uniform decline of infectious diseases and a uniform increase in non-infectious diseases should be modified: some infectious diseases decline significantly later (or slower) than others, whereas some 'degenerative and man-made diseases' declined significantly earlier. Her (age-adjusted) findings indicate, for example, that in the Netherlands mortality from stomach cancer already showed a decreasing trend at the beginning of the 20th century. Additionally, mortality from ischemic heart disease increased through most of the 20th century, but mortality from other heart diseases started to decline already in 1950. She stressed the need for a formulation of the epidemiological transition in terms of 'rise and decline of causes of death, and in terms of a disruption of health by emerging health risks and adaptation to health risks'.

Caldwell (2001) critiqued Omran's focus on the social and environmental change associated with modernisation, overlooking the importance of scientific inquiry and (the diffusion of) medical technology. Additionally, he commented on Omran's emphasis on the causes of death rather than on the changing patterns of disease. Although Omran's transition theory is primarily based on changes in the mortality proportions, Gage (2005) argued that it has resulted in the belief that the (age-specific) risk of degenerative diseases has increased (e.g., 'diseases of affluence' or 'Western diseases'), while this is not necessarily the case. He stressed that the widely held view that modern lifestyles and environments are bad for our degenerative health may be the result of secular improvements in data collection (e.g., reducing the proportion of unknown deaths) rather than a true deterioration in health; after correction for misclassification of causes of death, the degenerative disease mortality, and especially cardiovascular mortality, appears to have declined. However, the decline in infectious diseases was larger, resulting in an increasing proportion of degenerative disease mortality. Their conclusions are based on the assumption that large parts of the misclassified deaths (e.g., due to unknown and other diseases) are degenerative deaths. However, a Dutch study by Mackenbach (1993a) assumed that large proportions (circa 55-60\%) of the mortality decline in the category 'other/unknown diseases' is due to declines in infectious disease mortality. 


\subsubsection{Lessons learned}

The above sections show that the epidemiological transition theory has been accompanied by some important critiques and discussions. Nevertheless, it is generally believed that the epidemiological transition theory presents a broad conceptual framework that is useful for the study of trends in health, disease and mortality (den Draak, 2003). Mackenbach (1994), for example writes, that 'the epidemiological transition theory provides a potential powerful framework for the study of disease and mortality in populations.' Furthermore, he believes that the framework can be used to explore future changes. This is in line with Omran's (1998) comment that documenting and reflecting on the past can guide the future. Frenk et al. (1991) and den Draak (2003) describe what is perhaps the best way of looking at the 'epidemiological transition': not as a fixed theory, but rather as a framework or research paradigm.

So what can we learn from the epidemiological transition theory in order to improve our understanding of the broader context of population health. Based on the above, we have identified the following important lessons:

- Lesson 1: When the epidemiological transition theory was first formulated, it was implicitly assumed that all countries would follow the same pathway as the developed world. This rather deterministic view of population health was challenged, however, by the observation that the developing world does not only differ with regard to the timing and pace of the transition, but is moving to quite a different transition stage. Many argued that there is a risk of overgeneralisation. This becomes critically evident when comparing two very different countries like the Netherlands and Peru. In other words, the original theory did not account for the importance of the broader and complex context of population health. Hence, our exploration of future health should take into account that developing and developed countries differ in their transition experience.

- Lesson 2: The epidemiological transition theory shows that the system can respond in an unpredictable way. The re-emergence of infectious diseases in the developed world is a good example of such an unforeseen development; a few decades ago, the post-war successes in the developed world led to a view in the 1960s and 1970s that we were able to conquer infectious diseases so that they would no longer pose a serious threat to human health. In the context of this Ph.D. Thesis, this implies that multiple health futures are possible (contrary to the mere extrapolation of businessas-usual trends).

- Lesson 3: The causal mechanism behind the observed epidemiological changes cannot be explained using reductionist approaches. In response to the ongoing debate about the key driver of the epidemiological transition, it is increasingly argued that not one or two important factors triggered the vast improvements in our health, but the combination of reinforcing 
developments (e.g., nutritional improvements, personal hygiene, public health measures, health care) (Mackenbach, 1993a, 1993b). Murray and Chen (1993) argue that the mortality decline was due to an accumulation of health assets, linked to the process of modernisation involving changes in economic, cultural, political and social factors. Frenk et al. (1991) also acknowledge that the transitions in health and disease are determined in a multi-causal way. Harris (2004) defends McKeown's thesis, but argues that nutrition needs to be regarded as one of a battery of factors, often interacting. This is in line with our claim (Chapter 2) that a more integrated view of health determination -based on the complex and dynamic interplay among many causal factors- is important, and that such a system-based approach has gained more and more support over the past few years.

Hence, we argue that the epidemiological transition should be combined with a more holistic system-based approach. 


\section{Chapter 4}

\section{GLOBALISATION AND HEALTH: A CONCEPTUAL FRAMEWORK}

Based on:

- Huynen, M.M.T.E., Martens, P., \& Hilderink, H.B.M (2005). The health impacts of globalisation: a conceptual framework. Globalisation and Health, 1 , article number 14 (12 pages).

- Huynen, M.M.T.E., Martens, P., \& Hilderink, H.B.M (2005). The health impacts of globalisation: a conceptual framework. Bilthoven: Netherlands Environmental Assessment Agency (MNP-RIVM).

- Huynen, M.M.T.E., Martens, P. \& Hilderink, H.B.M. (2007). The diverse pathways from globalization to health. In: Global Forum Update on Research for Health Volume 4. Equitable access: research challenges for health in developing countries. London: Pro-Brook Publishing for the Global Forum on Health Research. 


\section{GLOBALISATION AND HEALTH: A CONCEPTUAL MODEL}

The pathways from globalisation to health are various and mediated by a multitude of factors like, for example, economic development, lifestyle and ecological changes. Two comprehensive frameworks concerning globalisation and health are the ones developed by Woodward et al. (2001) and by Labonte and Togerson (2002). The effects that are identified by Woodward et al. (2001) as most critical for health are mainly mediated by economic factors. Labonte and Torgerson (2002) primarily focus on the effects of economic globalisation and international governance. In our view, however, the pathways from globalisation to health are more diverse. Therefore, exploring the health effects of globalisation requires a more holistic approach than has previously been taken; it should be rooted in a broader conception of both population health and globalisation. This Chapter describes a new conceptual framework for the health implications of the globalisation process in the following steps:

1. Reviewing existing health models and identifying their strengths and weaknesses (section 4.1).

2. Identifying and structuring the main determinants of population health (section 4.2).

3. Identifying the main features of globalisation (section 4.3).

4. Constructing the conceptual model for globalisation and population health (section 4.4).

\subsection{REVIEW OF EXISTING HEALTH MODELS}

A review of existing health models forms the basis for the identification and structuring of the most important factors influencing health. We have selected several models that were developed for a wide range of purposes. This selection, representing the diversity of models, includes:

- Two models that were formulated in order to (quantitatively) explore future health: the Public Health Status and Forecasts (PHSF) model (Ruwaard \& Kramers, 1997) and TARGETS' population and health submodel (Rotmans \& de Vries, 1997).

- Two models that are widely accepted and used as conceptual frameworks of health determinants: developed by Dahlgren and Whitehead (1991), and developed by Evans and Stottart (1990).

- Two models that were formulated in order to explain the transition in public health status and mortality: developed by Frenk et al. (1991), and developed by Wolleswinkel-van den Bosch (1998).

- Two models that place human health in an ecosystem context: the Butterfly Model of Health (VanLeeuwen et al., 1999) and the Mandala of Health (Hancock \& Perkins, 1985; Hancock, 1993). 
- One model which applies a conceptual framework for complex issues to health: developed by Huynen and Martens (2002).

Public Health Status and Forecasts (PHSF) model (Ruwaard \& Kramers, 1993, 1997)1 In the Public Health Status and Forecasts (PHSF) model the identified determinants influence public health status, which, in turn, influences health care use. Public health status as well as developments in health determinants and health care determine health policy. Health policy has an indirect effect on health status via the determinants and a direct effect on health care utilisation. This whole process is influenced by demographic, macro-economic, socialcultural, and medical-technological autonomous developments. The model covers a comprehensive list of different determinants with a direct effect on health as well as a wide-ranging list of autonomous developments. In our view, however, these autonomous developments can better be perceived as more indirect (distal) determinants of public health, which can be influenced by policy measures in order to improve public health status. In the PHSF-model, this is, unfortunately, not the case. In addition, a link from health care use in the direction to health status is missing.

TARGETS' population and health module (Rotmans \& de Vries, 1997)

A key project within the 'Global Dynamics and Sustainable Development' program was the development of a mathematical global model called TARGETS (Tool to Assess Regional and Global Environmental and Health Targets for Sustainability). TARGETS consists of five interlinked sub-models, of which the 'Population and Health sub-model' includes a disease module simulating the process of being exposed to and dying of several health risks. However, the number of health determinants in this disease module is limited; they can be divided into socio-economic factors (Gross World Product and literacy status), environmental factors (food and water availability, and temperature increase), and lifestyle. The model does not distinguish between determinants with direct and indirect effects. The model includes a response module comprising water policy, food policy, health services and reproductive policies.

\footnotetext{
${ }^{1}$ In 2006, a revised adjusted PHSF model was published (de Hollander et al., 2006). In this revised model, the autonomous developments are now called external developments (demography, economy, social-cultural developments, technology and spatial factors), which are influenced by health (care) policy, policies in other domains and health status. In turn, these external developments influence the more direct health determinants (including health services). Additionally, a link from health care use (incl. prevention) in the direction to health status is now included.
} 
Model developed by Dahlgren and Whitehead (1991)

Dahlgren and Whitehead (1991) conceptualised the determinants of health diagrammatically as a number of layers of influence, each enveloping the previous one. This multi-layer model has become a widely used approach (see e.g., Acheson (1998) and IOM (2003)) and we think that the structure of different layers of influence is very appealing. However, there is some discrepancy between the layers in this model and the position of the selected health determinants in the causal chain. This model suggests that health is only directly influenced by the factors in the first layer, namely individual lifestyle factors. We believe that many other factors have a direct influence on health as well, like for example the availability of sufficient clean water or the quality of the living environment. Unfortunately, the model does not distinguish between determinants of different types of nature. Additionally, other response variables besides health care services are not included. It is, of course, possible that incorporating the various response options available to improve health was beyond the intended scope of this model.

\section{Model developed by Evans and Stottard (1990)}

Evans and Stottard (1990) present a conceptual model in order to construct a framework within which evidence on the determinants of health can be fitted, and which highlights the ways in which different types of factors and forces can interact. They constructed their model component by component, building on the 'health field concept' (Lalonde, 1981). Their model identifies several major fields of influence of health status and their interactions. However, the model does not distinguish between determinants of different levels of causality and it primarily focuses on factors with a direct influence on health. The response options include health care interventions and individual behavioural responses, but health(-related) policies are not taken into account.

Model developed by Frenk et al. (1991)

The purpose of the framework proposed by Frenk et al. (1991) is to organise conceptually the multi-causality of health conditions and systems in order to add a formulation about the determinants of health status to the health transition field. Their model is very comprehensive, but also rather complicated. They use two figures to clarify their framework: the first distinguishes between factors of a different nature, while the second figure explicitly distinguishes between factors of different analytical levels of causality. The model needs a lot of explanation and is, therefore, not very practical. The health care system included in the model compromises a wide range of health-promoting efforts such as diagnoses and treatment, health promotion, prevention, family planning, genetic counselling, occupational health services and environmental health services. However, the health care system is not explicitly included as a response, as the link from health status to 
the health care system is missing. Political institutions are also incorporated in this model, but the only policies resulting from these institutions seem to concern redistribution mechanisms affecting the level of wealth and social stratification.

Model developed by Wolleswinkel-van den Bosch (1998)

Wolleswinkel-van den Bosch (1998) describes a framework of determinants of mortality decline, consisting of two analytical levels: a proximal level and a distal level. Again, we think that a structure of different layers of causality is very compelling, but the model's structure is a bit too narrow in order to apply it to public health as it distinguishes only between two broad levels of causality with no differentiation between determinants of a different type/nature. Although political institutions are included in this model, explicit responses are not.

Butterfly Model of Health (VanLeeuzven et al., 1999)

The Butterfly Model of Health has been presented as a descriptive model for presenting and studying human health in ecosystems. Within the model, health-enveloped by biological and behavioural filters- is affected by both the biophysical (BP) and socio-economic (SE) environment. Health depends on the balance within and between BP and SE environments, and the ecosystems around them. This model does make a distinction between factors of a different nature: biological/behavioural filters, BP environments and SE environments. It acknowledges the influence of other ecosystems on the internal environments, but a more explicit distinction between different levels of causality is lacking. Even though political institutions are included in this model, explicit responses such as health policies or health-related policies are not.

The Mandala of Health (Hancock \& Perkins, 1985; Hancock, 1993)

The Mandala of Health is a model of the human ecosystem, which presents the influences on health by three circles or levels around the individual: the family, the community and human made environment, and finally, the culture and biosphere. Four subgroups of health influence are identified which impinge on the family and individual directly: personal behaviour, human biology, the physical environment, and the psycho-socio-economic environment. Individual health is subdivided into three parts: body, mind, and spirit. Hence, this approach makes a distinction between determinants of a different nature (subgroups of health influence) as well as between determinants of different levels of influence. However, there is some discrepancy between the layers in this models and the position of the selected health determinants in the causal chain. The model does include the medical system, which influences human biology and personal behaviour, but there is no reference to other response options. 
Table 4.1: Strengths and weaknesses of selected models.

Health Model
PHSF-model
(Ruwaard \&
Kramers, 1997)

TARGETS'
population and
health module
(Rotmans \& de
Vries, 1997)
Dahlgren and
Whitehead (1991)

Evans and

Stottart (1990)

Frenk et al. (1991)

Wolleswinkelvan den Bosch (1998)

Butterfly Model of Health

(VanLeeuwen et al., 1999)

Mandala of Health (Hancock \& Perkins, 1985;

Hancock, 1993)

SCENE applied to health (Huynen \& Martens, 2002)

\section{Strengths}

comprehensive list of determinants; distinction between determinants of a different nature

distinction between determinants of a different nature; several response variables

distinction between determinants of different levels of influence

distinction between determinants of a different nature

comprehensive; distinction between determinants of a different nature; distinction between determinants of different levels of influence; wide range of health-promoting efforts distinction between determinants of different causal levels

distinction between determinants of a different nature

distinction between determinants of a different nature; distinction between determinants of different levels of influence

distinction between determinants of a different nature

\section{Weaknesses}

indirect determinants are perceived as autonomous developments; health care use is not explicitly included as a response to health status

limited number of health determinants; no distinction between determinants of different causal levels

discrepancy between the layers in this models and the position of health determinants in the causal chain; no distinction between determinants of a different nature; only health care services included as response variable

no distinction between determinants of different causal levels; mainly direct determinants; health(-related) policies are not included as response too complicated; health-promoting efforts are not explicitly included as response to health status

limited number of determinants; no distinction between determinants of a different nature; no response variable no distinction between determinants of different causal levels; no response variable

discrepancy between the layers in this models and the position of health determinants in the causal chain; only medical system included as response variable no distinction between determinants of different causal levels; no response variable 
SCENE-model applied to health (Huynen \& Martens, 2002)

Huynen and Martens (2002) applied the structure of the SCENE-model (a conceptual framework for complex issues) (Grosskurth \& Rotmans, 2004) to population health and its determinants. This framework makes the traditional distinction between different forms of capital. However, its application to population health does not categorise the selected social-cultural, economic and environmental health determinants according to different levels of causality. Additionally, it does not explicitly include a response variable (e.g., policy measures), as institutional factors are included in the social-cultural domain.

To conclude, Table 4.1 summarises the identified strengths and weaknesses of the selected health models. Although this overview of existing health models is far from exhaustive and the selected models vary in purpose and content, their strengths and weaknesses reveal the following guidelines for an ideal-type of model of population health, which:

- makes a distinction between determinants of different types of nature;

- makes a distinction between determinants of different levels of causality;

- is as comprehensive as possible without becoming too complicated; and

- includes response variables/determinants.

\subsection{A NEW FRAMEWORK FOR POPULATION HEALTH}

The nature of the determinants and their level of causality can be combined into a basic framework that conceptualises the complex multi-causality of population health. In order to differentiate between determinants of a different nature, we will make the traditional distinction between institutional, sociocultural, economic, and environmental factors. These factors have different positions in the causal chain, and so operate at different hierarchical levels of causality (Figure 4.1). The chain of events leading to a certain health outcome includes both proximal and distal causes- proximal factors act directly to cause disease or health gains, and distal determinants are further back in the causal chain and act via (a number of) intermediary causes (WHO, 2002b). In addition, Figure 4.1 also distinguishes contextual determinants. These are the macro-level conditions that form the context in which the distal and proximal factors operate and develop. Determinants with different positions in the causal chain probably also differ in their temporal dimensions. Individual-level proximal health risks can be altered relatively quickly, for example by a change in personal behaviour; for disease rates in whole populations to change requires slower and more structural changes in contextual factors, often over the course of a few decades. 


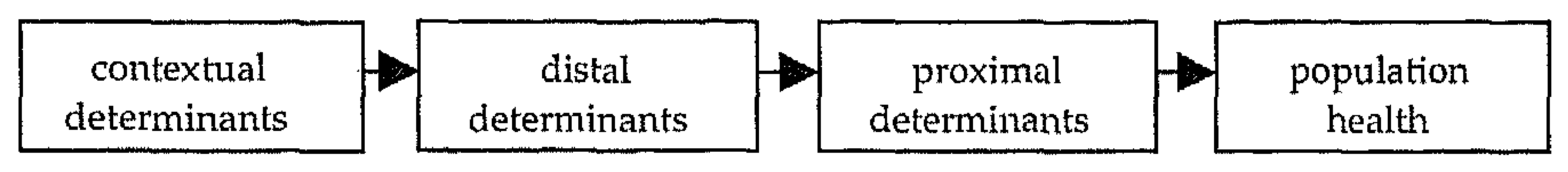

Figure 4.1: Population health determinants: different hierarchical levels of causality.

Further analysis of the selected health models and a extensive literature review resulted in a wide-ranging overview of the health determinants that can be fitted within this framework (Table 4.2). Figure 4.2, which draws on this analysis, shows a manageable number of general determinants, and includes important response variables such as health policies and health-related policies.

Table 4.2: Determinants of population health (Huynen et al., 2005b, 2005c).

$\begin{array}{lll}\text { Level/nature } & \text { General determinants } & \text { Detailed determinants } \\ \text { Contextual } & & \\ \text { Institutional } & \text { Institutional infrastructure } & \text { Governance structure } \\ & & \begin{array}{l}\text { Political environment } \\ \text { System of law }\end{array} \\ & & \text { Regulation } \\ \text { Economic } & \text { Economic infrastructure } & \text { Occupational structure } \\ & & \text { Tax system } \\ \text { Social-cultural } & \text { Culture } & \text { Markets } \\ & & \text { Religion } \\ & & \text { Ideology } \\ & & \text { Customs } \\ & \text { Population } & \text { Population size } \\ & & \text { Structure } \\ & & \text { Geographical distribution } \\ & \text { Social infrastructure } & \text { Social organisation } \\ & & \text { Knowledge development } \\ & & \text { Social security } \\ & & \text { Insurance system } \\ & & \text { Mobility and communication } \\ & & \text { Ecosystems } \\ \text { Eable continted on next page. } & \text { Climate } \\ & & \end{array}$


86 Chapter 4

Table 4.2 continued.

$\begin{array}{ll}\text { Level/nature } & \text { General determinants } \\ \text { Distal } & \text { Health policy } \\ \text { Institutional } & \text { Health-related policies } \\ & \\ \text { Economic } & \text { Economic development } \\ & \text { Trade } \\ \text { Social-cultural } & \text { Knowledge }\end{array}$

Social interactions

Environmental

Ecosystem goods and services

$\begin{array}{ll}\text { Proximal } & \\ \text { Institutional } & \text { Health services } \\ \text { Economic } & - \\ \text { Social-cultural } & \text { Lifestyle }\end{array}$

Physical living environment

\section{Detailed determinants}

Effective public health policy Sufficient public health budget Effective food policy Effective water policy Effective social policy Effective environmental policy Income/wealth Economic equity Trade in goods and services Marketing

Education and literacy Health education

Technology

Social equity

Conflicts

Travel and migration

Habitat

Information

Production

Regulation

Provision of and access to health services

Healthy food consumption patterns

Alcohol and tobacco use

Drug abuse

Unsafe sexual behaviour

Physical activity

Stress coping

Child care

Lifestyle related endogen factors (blood pressure, obesity, cholesterol levels)

Social support and informal care

Intended injuries and abuse/violence

Sufficient quality

Sufficient quantity

Sanitation

Quality of the living environment: biotic factors (e.g., disease pathogens), physical factors (e.g., temperature, radiation) and chemical factors (e.g., pollution)

Unintended injuries 


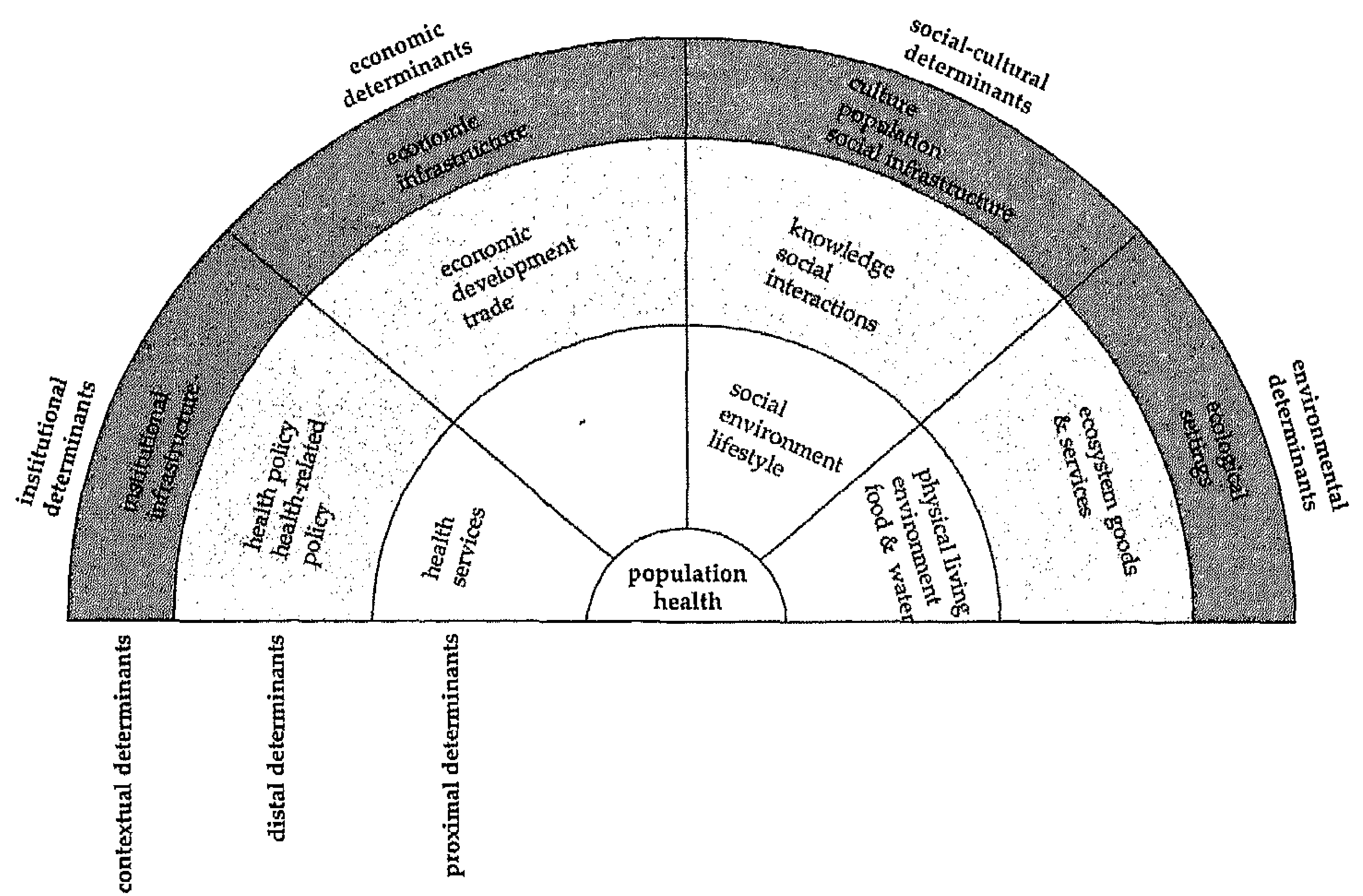

Figure 4.2: Multi-nature and multi-level framework of population health (Huynen et $a l ., 2005 b, 2005 c)$.

We must keep in mind, however, that the identified determinants interact along complex and dynamic pathways to 'produce' health at the population level; the health determination pathway is not unidirectional and several feedbacks are possible. There is interaction between and within determinants of a different nature and level of causality. To illustrate, economic inequity is related to education and social equity, while these factors are in turn related to lifestyle, which directly influence population health. Another example of the involved complexity: changes in health or health-related policies can be a response to problems with regard to determinants at the proximal level, indicating associations going from the proximal to the distal level. Additionally, health in itself can also influence its multi-level, multi-nature determinants; for example, ill health in itself can have a negative impact on economic development (WHO, 2001a). Below, the identified determinants of population health are discussed in more detail.

Proximal health determinants

As already explained, the determinants at the proximal level have a direct impact on population health. Health services are, of course, directly concerned with improving human health. In the social environment, the negative health effects of abuse and violence are important, as well as informal care and social 
support. The World Health Organization (WHO) (2002c) defines violence as 'the intentional use of physical force or power, threatened or actual, against oneself, another person, or against a group or community, that either result in or has a likelihood of resulting in injury, death, psychological harm, maldevelopment or deprivation'. It is estimated that in 20021.6 million people died worldwide as a result of self-inflicted, interpersonal or collective violence (WHO, 2002c). Lack of social support is believed to constitute an important risk for health and several studies confirm that psychosocial factors such as social relations and family environment are influencing our health (Hemmingway \& Marmot, 1999). Social relations can also underpin unhealthy behaviours via social influence (NPHP, 2001). This leads us to another social determinant at this level, namely lifestyle. It is already widely acknowledged and demonstrated that several modern behavioural factors such as an unhealthy diet, physical inactivity, smoking, alcohol misuse, and the use of illicit drugs are having a profound impact on human health (Box 4.1).

Box 4.1: Health and lifestyle.

Diet

Excess energy intake results, together with physical inactivity, in obesity. Obesity is an increasing health problem and has several co-morbidities such as non-insulin dependent diabetes and cardiovascular diseases (WHO, 2002a). The nutritional quality of the diet (e.g., fruit and vegetable intake, saturated versus unsaturated fats) is also very important for good health.

Inactivity

Physical inactivity has been linked to obesity, coronary hearth disease, hypertension, strokes, diabetes, colon cancer, breast cancer and osteoporotic fractures (WHO, 2002a).

Snoking

Tobacco is predicted to be the leading health risk factor by 2030 (WHO, 1999). It causes, for example, cancer of the trachea, bronchus and lung (WHO, 2002a), and cardiovascular diseases.

Alcohol use

The consumption of alcoholic beverages increases the risk of liver cirrhosis, raised blood pressure, heart disease, stroke, pancreatitis and cancers of larynx, oesophagus, stomach, liver and rectum (WHO, 2002a). The role of alcohol consumption in non-communicable disease epidemiology is, however, not straightforward. For example, small amounts of alcohol reduce the risk of cardiovascular diseases, while drinking larger amounts is an important cause of these very same diseases (Beaglehole \& Yach, 2003).

Illicit drugs

According to the World Health Report 2001. (WHO, 2001b), $0.4 \%$ of the total disease burden is attributable to illicit drugs (heroin and cocaine). Opiate users can have overall mortality rates up to 20 percent higher than those in the general population of the same age, due to not only overdoses but also to accidents, suicides, and infectious diseases such as AIDS (WHO, 2002a). 
Crucial for maintaining adequate health levels is the availability of sufficient quantities of adequate food and water. However, billions of people still lack access to basic water services; 1.4 billion people are without access to safe drinking water, while 2.3 billion are lacking sanitation systems necessary for reducing exposure to water-related diseases (Duda \& El-Ashry, 2000). As a result, an estimated 14 to 30 thousand people die each day from water related diseases (Hoekstra \& Huynen, 2002). Malnutrition is estimated to still be the single most important risk factor worldwide for disease, being responsible for $16 \%$ of the global burden in 1995, measured in Disability Adjusted Life Years (DALYs) (Murray \& Lopez, 1996). It even appears that the number of undernourished people in the developing world is no longer falling but climbing (FAO, 2003). In the physical living environment, the conditions in our direct living environment (e.g., housing, work, school) directly affect population health. The quality of our living environment is determined by biotic (e.g., disease pathogens), chemical (e.g., pollution) and physical (e.g., temperature, radiation, injuries) factors. Especially in the developing world infectious disease pathogens are still a major problem (Murray \& Lopez, 1997). One has to realise, however, that aspects of lifestyle (or behaviour) are important factors in exposure to the physical environment. For example, actual exposure to infectious disease pathogens in the environment is, to a large extent, determined by lifestyle factors such as unhygienic practices. The increased exposure to harmful UV-radiation by sunbathing is another example.

Box 4.2: Selected risk factors in the World Health Report 2002.

The World Health Report 2002 (WHO, 2002b) represents one of the largest research projects ever undertaken by the World Health Organization. The report describes the amount of disease, disability, and death in the world today that can be attributed to a selected number of the most important risks to human health.

The analysis in the report covered the following risk factors:

- Childhood and maternal undernutrition: underweight; iodine deficiency; iron deficiency; vitamin A deficiency; zinc deficiency; lack of breastfeeding.

- Other diet-related risk factors and physical inactivity: high blood pressure; high cholesterol; obesity, overweight, and high body mass; low fruit and vegetable intake; physical inactivity,

- Sexual and reproductive health: unsafe sex; lack of contraception.

- Addictive substances: smoking and oral tobacco use; alcohol use; illicit drug use.

- Environmental risks: unsafe water, sanitation and hygiene; urban air pollution; indoor smoke from solid fuels; lead exposure; climate change; traffic and transport.

- Selected occupational risks: work-related risk factors for injuries; work-related carcinogens; work-related airborne particulates; work-related ergonomic stressors; work-related noise.

- Other risks to health: unsafe health care practices; abuse and violence..

Clearly, many thousands of other threats to health exist within and outside the categories outlined above. These include very large causes of disease burden, such as risk factors for tuberculosis and malaria (which is currently responsible for $1.4 \%$ of global disease burden, with the vast majority of burden from this disease among children in sub-Saharan Africa). 
It is interesting to note that the majority of the risk factors selected by the WHO in their World Health Report 2002 (WHO, 2002b) (see Box 4.2) are proximal determinants and, therefore, they show great overlap with the proximal determinants identified in this Chapter (Table 4.3). Only climate change, selected by the WHO as a risk factor, cannot be categorised as a proximal determinant, although the direct effect of temperature increase or flooding can be classified under the physical living environment.

Table 4.3: Proximal health determinants and the selected risk factors in the World Health Report 2002.

\begin{tabular}{|c|c|c|}
\hline \multicolumn{3}{|c|}{ 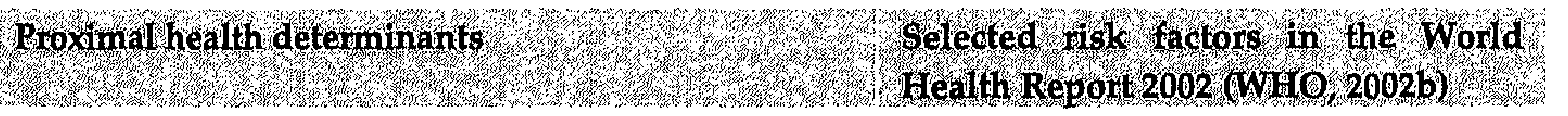 } \\
\hline $\begin{array}{l}\text { Health } \\
\text { services }\end{array}$ & $\begin{array}{l}\text { provision of and access to health } \\
\text { services }\end{array}$ & unsafe health care practices \\
\hline \multirow[t]{7}{*}{ Lifestyle } & food consumption patterns & low fruit and vegetable intake \\
\hline & tobacco and alcohol use, drug abuse & addictive substances \\
\hline & unsafe sexual behaviour & unsafe sex, lack of contraception \\
\hline & physical activity & physical inactivity \\
\hline & lifestyle related endogen factors & $\begin{array}{l}\text { obesity, high blood pressure, high } \\
\text { cholesterol }\end{array}$ \\
\hline & st & - \\
\hline & & lack of breastfeeding \\
\hline \multirow{3}{*}{$\begin{array}{l}\text { Social } \\
\text { environment }\end{array}$} & $d$ injuries & abuse and violence \\
\hline & and informal care & 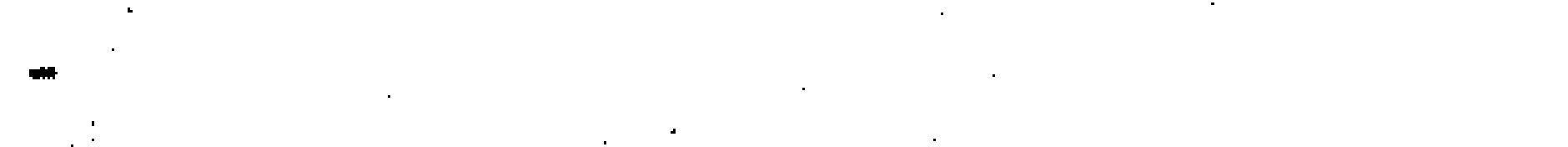 \\
\hline & intended injuries, a & - \\
\hline Food & sufficient quality/quantity & $\begin{array}{l}\text { lodine deficiency, iron deficiency, } \\
\text { vitamin A deficiency, zinc deficiency }\end{array}$ \\
\hline \multirow{3}{*}{$\begin{array}{l}\text { Water } \\
\text { Physical } \\
\text { environment }\end{array}$} & sufficient quality/quantity & unsafe water, sanitation and hygiene \\
\hline & quality of living environment & $\begin{array}{l}\text { indoor smoke from solid fuels, lead } \\
\text { exposure, urban air pollution, } \\
\text { occupational exposure to carcinogens, } \\
\text { airborne particulates, noise, ergonomic } \\
\text { stressors }\end{array}$ \\
\hline & unintended injuries & $\begin{array}{l}\text { occupational injuries, traffic and } \\
\text { transport }\end{array}$ \\
\hline
\end{tabular}

\section{Distal determinants}

Institutional factors at the distal level include, of course, health policy and health-related policy (food policy, water policy, social policy, environmental policy, etc.). Economic development is also an important distal determinant; economic development has enhanced health and life expectancy in many populations; gross national product per capita correlates strongly with national health status (Pritchett \& Summers, 1996). Wealth affects health in a number of 
ways, for example by bringing about improvements in the quality and quantity of food and water, by instituting effective public health measures (including medical care), and by leading to improvements in literacy and the physical environment (Powles, 1992). However, the relationships between economic development and health are not always straightforward: for example, despite high income levels, the Middle Eastern oil producing countries have a relatively low life expectancy, whereas countries such as China and Sri Lanka are 'healthier' than their per capita income would lead one to expect (UNDP, 1999). In addition, economic inequity within and between populations plays an important role, for it induces absolute poverty in much of the world's population, thereby exacerbating health-related problems. Economic development has also been accompanied by increased trade. The links between trade and disease have been recognised for centuries, as is demonstrated by the fact that the path of the Black Death in the 14th century followed the international trading routes (Bettcher et al., 2000). Increasing global food trade, for example, creates new opportunities for infections to flourish due to the movement of contaminated food products or the shipment of livestock. Global food trade is also accompanied by global marketing and by altered eating habits.

Health issues related to social interactions include conflicts, travel and migration, and social equity. Modern warfare affects public health directly through the soldiers and civilians who die or are injured in fighting (Yassi et al., 2001). However, there are also indirect health impacts of conflicts through effects on, for example, the economic system, food and water availability, the provision of health care, welfare, and adverse impacts on the environment. Warfare can also create a hospitable environment for infections in many ways (Louria, 2000). Garfield and Neugut (1997) suggest that civilian deaths compose $90 \%$ of all deaths in twentieth century wars. Travel and migration is a potent force in the emergence of disease. When humans travel, they carry their genetic makeup, immunologic sequelae of past infection, cultural preferences, customs and behavioural patterns, while microbes, animals and other biological life also accompany them. People also change the environment when they travel or migrate; introduced technology, farming methods, deforestation, dam-building, opening of new roads, treatment and drugs, chemicals, and pesticides all may have great health impacts (Wilson, 1995). Health is also closely related to knowledge; people and households with more education enjoy better health. Even independent of wealth, improvements in education - particularly women's education - have a considerable bearing on improvements in family health. Unfortunately, in many poor countries, levels of literacy and education are still very low. Technological and scientific knowledge, and proper health education are also important factors.

Environmental determinants at the distal level are ecosystem goods and services. Ecosystems (contextual layer of influence) provide us with the basic 
necessities and essential services, which are the result of the natural processes within the ecosystem. De Groot $(1992,1999)$ defined ecosystem functions as the capacity of natural processes and components to provide goods and services that satisfy human needs (including good health). In fact, we need to recognise the 'social dependence' on ecosystem services (Daily, 1997). In today's world, several environmental threats compromise the provision of ecosystem goods and services, like climate change, loss of biodiversity and land use changes.

\section{Contextual determinants}

Contextual determinants concern the macro-level conditions, which shape the distal and proximal health determinants. At this level, the institutional infrastructure (governance structure, political environment, system of law, regulation) and economic infrastructure (occupational structure, tax system, markets) can be identified. In the social domain, we distinguish between culture (ideology, religion, customs), population (size, structure, distribution), and social infrastructure (social organisation, knowledge development, social security, insurance system, mobility and communication). Contextual environmental determinants encompass the ecological setting (e.g., ecosystems, biodiversity, climate).

However, the direction (positive or negative) of the relationship between most contextual factors and human health cannot be easily determined. For example, the effectiveness of certain governance structures is determined by the background of the health problems. The same is true for the effectiveness of 'social organisation'. Another example is the fact that some customs have negative health implications (e.g., circumcision of women), while others are more beneficial (e.g., a diet rich in unsaturated fats). Other contextual aspects might have a somewhat more unidirectional association with health such as social security, although these still have to be viewed against a background of the existing societal context.

\subsection{FEATURES OF GLOBALISATION}

More and more scholars agree that globalisation is an extremely complex phenomenon. The globalisation process is characterised by a growing intensity, extensity and velocity of institutional, economic, social-cultural and ecological interactions, resulting in transborder processes and effects (see section 1.2.2.). However, the identification of all possible health effects of the globalisation process goes far beyond the current capacity of our mental ability; due to our ignorance and interdeterminacy of the global system that may be out of reach forever (Martens \& Rotmans, 2002). In order to focus our conceptual framework, we distinguish- with the broader definition of globalisation in mind- the following important institutional, economic, socio-cultural, and 
ecological features of the globalisation process: (the need for) new global governance structures, global markets, global communication and diffusion of information, global mobility, cross-cultural interaction, and global environmental changes (Table 4.4).

Table 4.4: Features of globalisation (Huynen et al., 2005b, 2005c).

\begin{abstract}
Features of globalisation
New global governance structure

Global markets

Global communication and diffusion of information

Global mobility

Cross-cultural interaction

Global environmental changes

Globalisation influences the interdependence among nations as well as the nation state's sovereignty leading to (a need for) new global governance structures.

Globalisation is characterised by worldwide changes in economic infrastructures and the emergence of global markets and a global trading system.

Globalisation makes the sharing of information and the exchange of experiences around common problems possible.

Global mobility is characterised by a major increase in the extensity, intensity and velocity of movement and by a wide variety in 'types' of mobility.

Globalising cultural flows result in interactions between global and local cultural elements.

Global environmental threats to ecosystems include global climate change, loss of biodiversity, global ozone depletion and the global decline in natural areas.
\end{abstract}

Nezw global governance structures

Governance is concerned with the manner in which an organisation or society steers itself (Rosenau, 1995) and its structure involves the interaction between the state, private sector and civil society (UNDP, 1997). Global health governance concerns the collective forms of governance, from the sub-national to global level, which address health issues with global dimensions (Lee \& Goodman, 2002). Obviously, good (global) health governance is necessary to deal with the health implications of globalisation. On the other hand, however, many believe that globalisation itself is affecting governance and challenges the governments' ability to deal with health threats to their population; it influences the interdependence among nations as well as the nation state's sovereignty (Box 4.3). As a result, current governance structures have become inefficient and ineffective as the changes in our social and economic reality have outpaced change in the (political) institutions (Reinicke, 1998) and nations no longer represent truly independent, sovereign countries (Frenk et al., 1997). From a health perspective, international health issues threaten external sovereignty, while transborder health problems compromise internal sovereignty. 
In order to be able to conduct effective policy, there is a need for new institutional arrangements and a new form of global health governance. The need for new institutions and regulations is, for example, demonstrated by the fact that global economic competition may persuade countries to avoid the costs of social welfare in order to be more competitive (social dumping) unless supranational or global regulations are in place that discourage this (Deacon, 1997). International health organisations seem to be the ideal vehicle to start dealing with health problems that go beyond the capacity of national systems. However, the existing health(-related) institutions for multilateral cooperation are experiencing inefficient overlap, while at the same time other crucial responsibilities are not carried out at all. In addition, new actors in the health policy arena like $\mathrm{NGO}^{\prime}$ 's and transnational organisation are gaining prominence (Frenk et al., 1997). The current loose set of institutions needs to develop into effective networks of governance and consensus has to be achieved about the core functions of international (health) organisations (Deacon, 1997).

\title{
Box 4.3: Globalisation and the nation state's sovereignty.
}

\begin{abstract}
Internal sovereignty concerns the relationship between a government and society at large. In operational terms, internal sovereignty in today's democracy means the ability of a government to formulate, implement and manage public policy; governments exercise internal sovereignty when they make laws and regulations, decide on the best and most effective ways to implement those laws and regulations and monitor compliance with them. A threat to a countries operational internal sovereignty implies a threat to its ability to conduct public policy (Reinicke, 1998). Because new global forces have eroded national borders, national regulations lose their grip on national societies in multiple policy domains. For example, transnational cooperations control a large share of the world's capital and currencies leap from one financial market to the next, often defying national regulation (Frenk et al., 1997). In contrast to internal sovereignty, where the state is the central authority, external sovereignty has as its main characteristic the absence of central authority; external sovereignty implies the independence of states in the international system (Reinicke, 1998). Keohane and Ney (1977) defined the term complex interdependence to characterise a condition in which independent states are connected by an increasing number of channels- political, social, economic, cultural and others. It implies sensitivity to an external force (e.g. the Asian financial crisis affected economies worldwide) and the growing interdependence challenges a nation's external sovereignty.
\end{abstract}

\section{Global markets}

Globalisation is characterised by worldwide changes in economic infrastructures. One of the main drivers of these changes is the emergence of capitalism (Rennen \& Martens, 2003), which is based on a free market, open competition, profit motive, private ownership and minimal government interference. In addition, significant improvements in transport and communication networks also contributed to the infrastructure for a global trading system and the institutionalisation of trade liberalisation provided the 
basis for open worldwide markets (Held et al., 1999) and the integration of the world's economies (Reinicke, 1998). One could perhaps even speak about a 'global market', which is characterised by, for example, global products, fragmentation of production, global sales strategies, global trading systems, global currencies, and the proliferation of transborder corporate networks.

\section{Global communication and diffusion of information}

The globalisation process facilitates the sharing of knowledge and makes the exchange of experiences around common problems possible, especially as information is more and more perceived as a global public good (Kaul et al., 1999). Whether it concerns the transfer of information in written or spoken form or via images (e.g., television), communication networks have acquired a global reach. Additionally, English has become a global lingua franca and has served as the chief medium of verbal communication in international relations (Scholte, 2000). As a result, the worldwide diffusion of know-how, knowledge and technological expertise is growing and has become increasingly important (Archibugi \& Lundvall, 2001). Many innovations in communications technology have transformed the capacity, costs, speed and complexity of telecommunications systems (Held et al., 1999) and spurred the emergence of global communication networks and global communication tools. The Internet, for example, came into widespread use in the 1990s via implementation of World Wide Web and is now facilitating electronic communication across the globe. The 'globalisation of knowledge' even goes beyond the exchange of knowledge across national boundaries, and implies that the production of knowledge will be organised on a global scale involving international collaborations across greater geographical distances. Furthermore, the extended range of communication possibilities could also help to address the interface between science and the general public. The globalisation process also facilitates the diffusion of (medical) technology (Box 4.4), although there are also some new barriers to the spread of knowledge like Trade Related Intellectual Property rights (TRIPS).

\section{Global mobility}

Mobility broadens our horizons and enables us, for example, to establish new contacts, to gain new experiences, and to increase our knowledge. Global mobility is characterised by a major increase in the extensity, intensity and velocity of movement and by a wide variety in 'types' of mobility. Improved infrastructures, institutions of transport, communication and regulation (Held et al., 1999) have resulted in the current unprecedented volume, speed, and reach of travel (Wilson, 1995). During the 20th century, the motorised movement of people increased more than one hundredfold (OECD, 2000b). The expanding mobility is also demonstrated by the fact that there is almost no 
populated place on earth that cannot be reached by a migrant or product within 1-2 days of travel (Macpherson, 2001).

Box 4.4: A taxonomy of the globalisation of technology (Archibugi \& Michie, 1995; Archibugi \& Iammarino, 2000).

The aim of this taxonomy is to classify individual innovations according to the ways in which they are produced, exploited and diffused internationally in order to help to identify the several forms of the 'globalisation of technology'. Three main categories can be distinguished:

- The international exploitation of nationally produced technology: This includes the attempts of innovators (firms and individuals) to obtain economic advantage by exploiting their technological competences in markets other than the domestic one (e.g., exports of innovative goods, sale of licenses and patents, foreign production of innovative goods that are internally generated).

- Global generation of innovations: This includes innovation generated by single proprietors (multinational enterprises) on a global scale. The authentic global generation of innovations requires the existence of inter-national but intra-firm Research and Development (R\&D) centres.

- Global technological collaborations: This form of knowledge transmission emerged in the academic world, which always had a transnational spectrum of action: knowledge is disseminated without always requiring financial compensation (e.g., joint scientific projects and networks, scientific exchange, sabbatical years, international flow of students). Technological collaboration also occurs when two different firms (national or multinational) decide to establish joint-ventures with the aim of developing knowledge and/or products.

\section{Cross-cultural interaction}

Many developments in the globalisation process are causing worldwide changes in culture, but does this mean that a unified world culture is emerging? Some even speak about cultural imperialism or 'McDonaldisation' (Ritzer, 1993, 1998). This perspective is based, however, on the assumption that western cultural elements are uncritically absorbed in non-western nations and that cultural inflows are suppressing of existent local meanings and forms (Schuerkens, 2003).

A second theory refers to cultural differentialism or lasting difference. According to this view, the future will be characterised by a mosaic of immutably different cultures and civilisations (Nederveen Pieterse, 2004). Huntington (1993) argues that world politics is entering a new phase and that the fundamental source of conflict in this new world order will be cultural. The increasing interaction between peoples of different civilisations will 'intensify civilisation consciousness and awareness of differences between civilisations and commonalities within civilisations'. He envisions that 'civilisation identity will be increasingly important in the future' and that the world will be shaped in large measure by the interactions among seven or eight major civilisations (i.e., cultural entities), which include Western, Confucian, Japanese, Islamic, 
Hindu, Slavic-Orthodox, Latin-American, and possibly African civilisation. However, Huntington's views have given rise to extensive debates (Nederveen Pieterse, 2004).

A third theory argues that local cultures are more robust and adaptive than the rhetoric of globalisation would have us believe: a well-established viewpoint among social-cultural scientists considers globalisation as a process of hybridisation (Nederveen Pieterse, 2004). Cultural hybridisation refers to processes of local absorption of cultural flows and the mixture between global and local cultural elements. Inflowing cultural elements, such as television series, western consumer articles and values introduced by migrants, can become elements of the local daily life, often in changed forms and adapted to the local context (Schuerkens, 2003). Hannertz (1996) argues, for example, that the local is the area 'where the global, or what has been local somewhere else, also has some chance of making itself at home'.

\section{Global environmental changes}

The health of the people and that of the environment are intimately interlinked. The WHO estimated that the environmental contribution to the global burden of disease and injury is approximally $23 \%$ (Yassi et al., 2001). Pollution affects health at the more proximal level, although the spread of the pollution or its sources could have a more global character. Several other global environmental issues threaten our health more indirectly by posing risks to the world's ecosystems and, subsequently, to the provision of ecosystem goods and services. These global environmental changes, caused by a combination of population growth and modern production and consumption patterns, are affecting our health on a global scale. For example:

- climate change is not restricted to the geographical areas of the polluting sources and its health effects are expected to be widespread around the globe;

- the loss of biodiversity often has transborder causes and could result in widespread health effects;

- the decline in natural areas is also an important global environmental problem as the natural systems of our planet are increasingly competing with the human-made environment (e.g., cropland, cities) due to anthropological changes in land cover and land use.

In today's world, it becomes more and more clear that anthropogenic ecological changes have acquired a global dimension (Scholte, 2000) and that humans are increasingly polluting and degrading the global commons. In addition, poor societies have a relatively modest per capita consumption of resources, but as globalising countries in the developing world are increasing their economic activity and consumption patterns they will increase their impact on the environment as well (Frenk et al., 1997). 


\subsection{CONCEPTUAL FRAMEWORK FOR GLOBALISATION AND HEALTH}

This section builds on the previous paragraphs and develops a conceptual framework for the effects of globalisation on population health. We have identified (the need for) global governance structures, global markets, global communication and the diffusion of information, cross-cultural interaction, global mobility, and global environmental changes as important features of globalisation. Based on section 4.2, it can be concluded that these features all operate at the contextual level of health determination and influence distal factors like health(-related) policy, economic development, trade, social interactions, knowledge, and the provision of ecosystem goods and services. In turn, these changes in distal factors have the potential to affect the proximal health determinants. Our conceptual framework for globalisation and health, subsequently, links the above-mentioned features of the globalisation process with the identified health determinants. This exercise results in Figure 4.3.

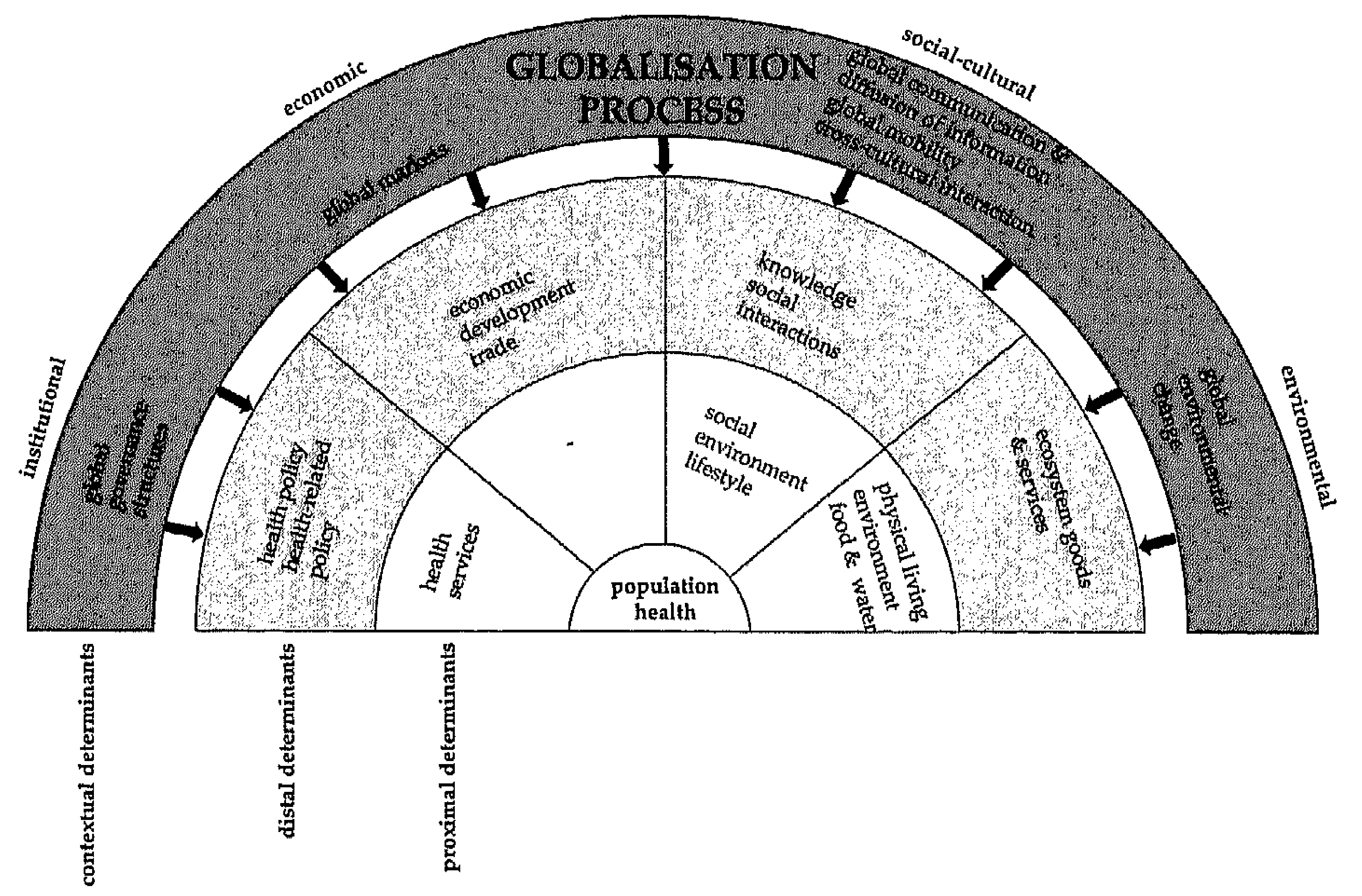

Figure 4.3: Conceptual framework for globalisation and population health (Huynen et al., 2005b, 2005c). 
Globalisation and health: a conceptual framework | 99

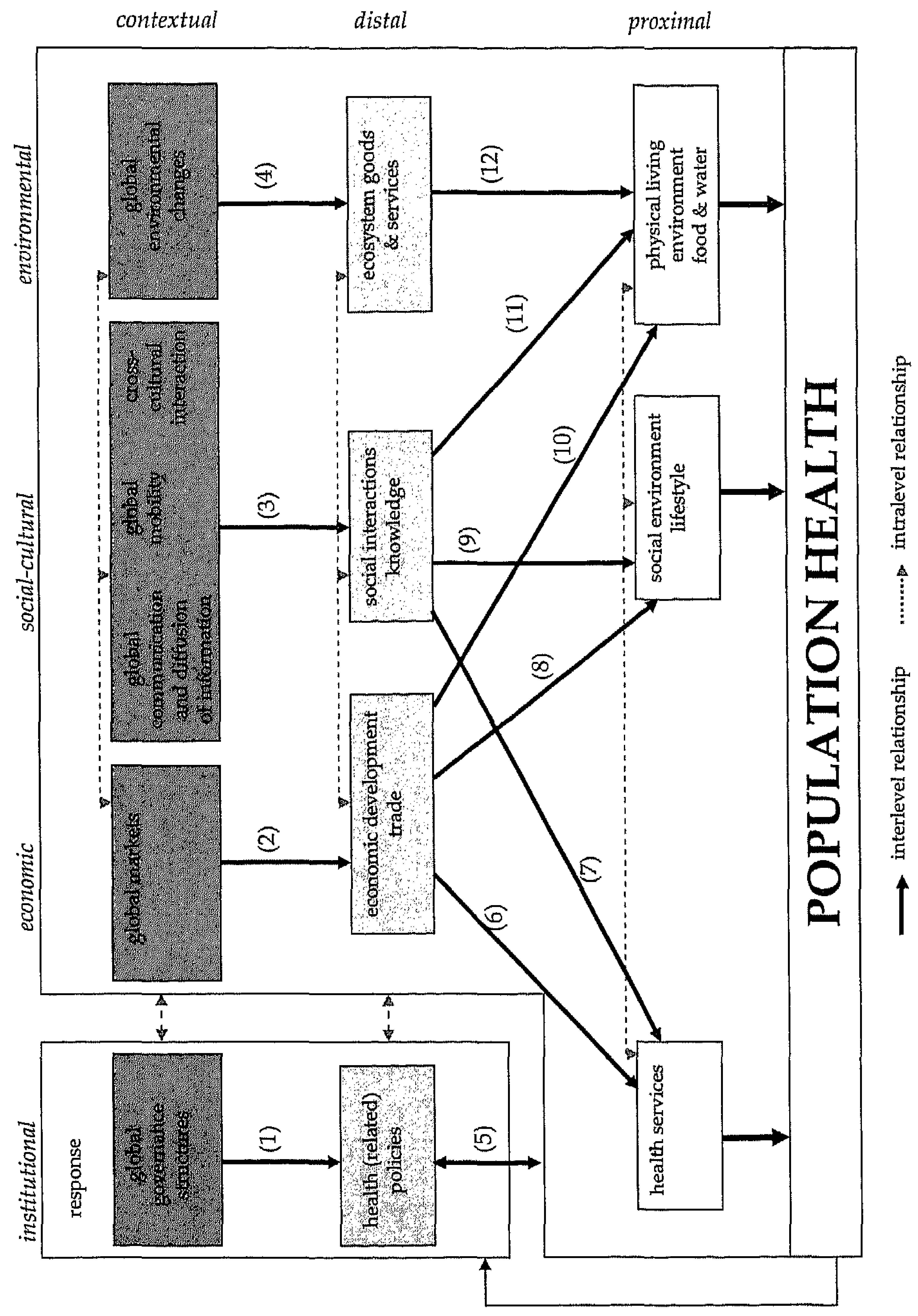

Figure 4.4: Conceptual model for globalisation and population health (Huynen et al., 2005b, 2005c). 
Figure 4.4, subsequently, shows that within the developed framework, several links between the specific features of globalisation and health can be derived. These important links between globalisation and health are discussed in the following paragraphs. It is important to note that Figure 4.4 primarily focuses on the relationships in the direction from globalisation to health. This does not mean, however, that globalisation is an autonomous process. One has to keep in mind that globalisation is influenced by many developments at the other levels, although these associations are not included for reasons of simplification. As a result of the complex interplay by all factors in the systems, multiple future globalisation pathways are possible (see also Chapter 5).

\subsubsection{Globalisation and distal health determinants}

Figure 4.4 shows that the processes of globalisation can have an impact on all identified distal determinants (Figure 4.4; arrows 1-4). Below, the implications of globalisation on these distal determinants will be discussed in more detail.

Health(-related) policies

Global governance structures are gaining more and more importance in formulating health(-related) policies (Figure $4.4 ;$ arrow 1). According to Dodgson et al. (2002), the most important organisations in global health governance are the WHO and the World Bank (WB). The latter plays an important role in the field of global health governance as it acknowledges the importance of good health for economic development and focuses on reaching the Millennium Development Goals (MDGs) (UN, 2005). The WB also influences health(-related) policies together with the International Monetary Funds (IMF) through the Structural Adjustment Programmes (SAPs) (Box 4.5). In order to give a more central role to pro-poor growth considerations in providing assistance to low-income countries, the IMF and WB introduced the Poverty Reduction Strategy approach in 1999 (IMF, 2004).

In addition, the policies of the World Trade Organization (WTO) are also increasingly influencing population health (Commission on the Future of Health Care in Canada, 2002; Hong, 2000; Walt, 2000; WHO \& WTO, 2002). The WTO was formed in January 1995, and governs international trade. The organisation contains a framework for enforcement of rights and obligations to more than 20 international trade agreements (Hong, 2000). Besides the trade in goods, these agreements also cover the trade in services and intellectual property (Box 4.6. and Box 4.7). Fidler (2002) argues that 'from the international legal perspective, the centre of power for global health governance has shifted from WHO to the WTO'. Opinions differ with regard to whether the WTO agreements provide sufficient possibilities to protect the population from the 
adverse (health) effects of free trade or not (Singer, 2002). In 2002, the WTO ruled that the French ban on the import of all products containing asbestos was legal on health grounds, despite protests from Canada (WTO, 2000, 2001). However, protecting citizens against health risks in cases of scientific uncertainty is still difficult, as the WTO is reluctant to accept precautionary trade restrictions (Mbengue \& Thomas, 2005).

\section{Box 4.5: Structural Adjustment Programmes (SAPs) and health.}

The World Bank (WB) and the International Monetary Fund (IMF) introduced Structural Adjustment Programmes (SAPs) in order to promote efficiency and a more practical allocation of productive resources based on market mechanisms. The SAPs-packages include massive deregulation, privatisation, currency devaluation, social spending cuts, lower corporate taxes, export driven strategies and removal of foreign investment restrictions (Hong, 2000). Introducing SAPs was a precondition for 'soft loans' to indebted countries, while other more advanced developing countries voluntarily introduced SAPs (Balasubramaniam, 2000). It is argued that the implementation of SAPs had adverse effects on multiple health determinants and the restructuring of the health sector has led to the 'collapse of preventive and curative care', while user fees in health care has resulted in 'the exclusion of large sectors of the population from health services'. In addition, cuts in public expenditure caused a drastic decline in control and prevention measures and, as a result, diseases are re-emerging (Hong, 2000).

Box 4.6: Trade Related Intellectual Property rights (TRIPs) and health.

The World Trade Organization's Trade Related Intellectual Property rights, or TRIPs, oblige member states to provide a minimum 20-year term monopoly patent protection on drugs. Many health advocates are convinced that this system drives up drug costs, restricts the availability of affordable medicines (Commission on the Future of Health Care in Canada, 2002), affects the spread of medical knowledge and will shift research and innovation from science to the corporate business (Hong, 2000). In order to a balance between the longer-term objective to provide incentives for future inventions and the shorter-term objective to allow people to use existing ones, the agreement does provide some flexibilities for governments in order to protect public policy objectives, provided certain conditions are fulfilled (WHO \& WTO, 2002). Article 31 allows countries to grant a license to copy the patented drugs to domestic pharmaceutical suppliers without permission from the patent holder for reasons of public interest. This compulsory drug licensing is allowed as long as the drug is intended for use in the domestic market and royalties still have to be paid to the patent holder (WHO \& WTO, 2002). The U.S. have already used this option many times to restrict the monopoly rights of the patent holder in the interest of the public good (Hong, 2000; Walt, 2000). Despite the flexibilities granted by the TRIPs agreement, few developing countries have employed them to develop policies to protect public health (Martin et al., 2007). It has been argued that developing countries have been pressured not to provide compulsory licensing. And although Article 27.3(a) allows member states to exclude diagnostic, therapeutic and surgical methods from patentability, pressure is expected from the industry to expand their rights in this area (Hong, 2000). 
Box 4.7: General Agreement on Trade in Services (GATS) and health.

The WTO service agreement known as the General Agreement on Trade in Services (GATS) sets out rules governing international trade in almost all services. A total of 160 service sectors are covered by GATS, of which some are essential social services concerning public goods (Anomynous, 2001). However, each country can choose in which service sectors they want to make GATS commitments. Health services and social services are lacking behind with regard to the rate they are being listed as open to competition, but still 40 percent of the WTO members have made some type of commitment on health services (e.g., hospital management) (WHO \& WTO, 2002). Under Article XIV of GATS, WTO members are entitled to take measures necessary to protect human health, regardless of their obligations under the agreement. However, affected WTO countries may in turn challenge such measures if they feel that the relevant provisions have not been respected (WHO \& WTO, 2002). In the mean time, little information exists on the economic benefits of GATS or its social and environmental effects, and it is argued that the major traders in the industrial world are to gain most due to their far greater capacity to supply services (Anomynous, 2001).

Another important development is the growing number of public-private partnerships for health, as governments increasingly attract private sector companies to undertake tasks that were formerly the responsibility of the public sector. At the global level, public-private partnerships are more and more perceived as a possible new form of global governance (Walt, 2000) and could have important implications for health polices, but also for health-related policies.

\section{Economic development}

Opinions differ with regard to the economic benefits of economic globalisation (Figure 4.4; arrow 2). On the one side, 'optimists' argue that global markets facilitate economic growth and economic security, which would benefit health. They base themselves on the results of several studies that conclude that inequities between and within countries have decreased due to globalisation (e.g., Frankel (1999), Ben David (2000), Dollar and Kraay (2001)). Additionally, it is argued that, although other nations or households might become richer, absolute poverty is reduced and that this is beneficial for the health of the poor (Feachem, 2001). On the other side, 'pessimists' are worried about the health effects of the global market. Baum (2001) states that the current forms of globalisation are making the world a safe place for unfettered market liberalism and the consequent growth of inequities (...) posing severe threats to people's health'. The 2005 Human Development Report (UNDP, 2005) argues that one of the prevailing 'myths' of economic globalisation is that open markets will result in an era of convergence; trade liberalisation 'has done little to slow down the marginalisation of Sub-Saharan Africa' and the current 'trading system favours the developed world'. In fact, notwithstanding some spectacular growth rates, 
especially in East Asia, incomes declined between 1965 and 1997 in 16 of the world poorest countries (Melchior et al., 2000).

\section{Trade}

Due to the establishment of global markets and a global trading system, there has been a continuing increase in world trade (Figure 4.4; arrow 2). According to the WTO, total trade multiplied by a factor 14 between 1950 and 1997 (WTO, 2003). More recently, the year 2004 saw an impressive growth in trade, which exceeded average growth recorded over the preceding decade (WTO, 2005). Today all countries trade internationally and they trade significant proportions of their national income. The array of products being traded is wide-ranging; from primary commodities to manufactured goods. Besides goods, services are increasingly being traded as well (Held et al,, 1999). In addition to legal trade transactions, illegal drug trade is also globalising, as it circumvents national and international authority and takes advantage of the global finance systems, new information technologies and transportation.

\section{Social interactions: migration}

Due to the changes in the infrastructures of transportation and communication, human migration has increased at unprecedented rates (Figure 4.4; arrow 3) (Held et al., 1999). According to Held et al. (1999) tourism is one of the most obvious forms of cultural globalisation and it illustrates the increasing timespace compression of current societies. However, travel for business and pleasure constitutes only a fraction of total human movement. Other examples of people migrating are missionaries, merchant marines, students, pilgrims, militaries, migrant workers and Peace Corps workers (Held et al., 1999; Wilson, 1995). Besides these forms of voluntary migration, resettlement by refugees is also an important issue. The UN Population Division estimates the global migrant population in 2005 at between 185-192 million people (UN, 2003a). However, the concerns regarding the economic, political, social and environmental consequences of migration are growing (UN, 2004), and many governments are moving towards more restrictive immigration policies.

\section{Social interactions: conflicts}

In the aftermath of the terrorist attacks on September $11^{\text {th }} 2001$, many questioned the possible links between globalisation and the risk of conflicts. On the one side, globalisation can decrease the risk of tensions and conflicts, as societies become more dependent on each other due the worldwide increase in global communication, global mobility and cross-cultural interactions (Figure 4.4; arrow 3). Others argue that the resistance to globalisation has resulted in religious fundamentalism, and worldwide tensions and intolerance (Frenk et al., 1997). According to Huntington (1993), increasing cross-cultural interactions will result in cultural differentialism and the 'clash of civilisations'. Besides the 
debates about culture and identity, however, other factors also need to be considered. Nassar (2005) describes globalisation as a process that leads to a 'migration of dreams' in which the world's poor are able to learn of the luxuries of the western world. The increased degree of relative deprivation results in growing tensions. In addition, Zwi et al. (2002) identify several other factors that are associated with both globalisation and the risk of conflicts, such as increased global trade in arms, and inadequate policies. It can, therefore, be concluded that globalisation-induced changes in other factors at the distal level play an important role.

Social interactions: social equity and social networks

Cultural globalisation (global communication, global mobility, cross-cultural interaction) can also influence cultural norms and values about social solidarity and social equity (Figure 4.4; arrow 3). It is feared that the self-interested individualism of the marketplace spills over into cultural norms and values resulting in increasing social exclusion and social inequity. Exclusion involves disintegration from common cultural processes, lack of participation in social activities, alienation from decision-making and civic participation and barriers to employment and material sources (Reid, 2004). On the other hand, however, the geographical scale of social networks is increasing due to global communications and global media. The women's movement, the peace movement, organised religion and the environmental movement are good examples of such transnational social networks. Besides these more formal networks, informal social networks are also gaining importance, as like-minded people are now able to interact at a distance through, for example, the Internet. In addition, the global diffusion of radio and television plays an important role in establishing such global networks (Held et al., 1999). The digital divide between poor and rich, however, can result in social exclusion from the global civil society.

\section{Knowledge}

The knowledge capital within a population is increasingly affected by global developments in communication and mobility (Figure 4.4: arrow 3). The term 'globalisation of education' suggests getting education into every nook and cranny of the globe. Millions of people now acquire part of their knowledge from transworld textbooks. Most colleges and universities are able to work together with academics from different countries, students have many opportunities to study abroad, and 'virtual campuses' have been developed. In addition, television, film and computer graphics have greatly enlarged the visual dimensions of communication. Many people today 'read the globalised world without a book' (Scholte, 2000). Overall, it is expected that the abovediscussed developments will also improve health training and health education (e.g., Feachem (2001) and Lee (1999a)). 
Ecosystem goods and services

Global environmental changes can have profound effects on the provision of ecosystem goods and services to mankind (Figure 4.4; arrow 4). The Intergovernmental Panel on Climate Change (IPCC) (2001a) concludes that it is expected that climate change can result in significant ecosystem disruptions and threatens substantial damage to the earth's natural systems. In addition, several authors have addressed the link between biodiversity and ecosystem functioning and it is agued that maintaining a certain level of biodiversity is necessary for the proper provision of ecosystem goods and services (Chapin et al., 2000; Schulze \& Mooney, 1994; Schwartz et al., 2000; UNEP, 1995). However, it is still unclear which ecosystem functions are primarily important to sustain our physical health. Basically, the following types of 'health functions' can be distinguished. First, ecosystems provide us with basic human needs like food, clean air, clean water and clean soils. Second, they prevent the spread of diseases through biological control. Finally, ecosystems provide us with medical and genetic resources, which are necessary to prevent or cure diseases (Huynen et al., 2004). The Millennium Ecosystem Assessment (Corvalan et al., 2005) warns that the ongoing degradation of ecosystem functions poses a growing health risk.

Importance of intra-level relationships at the distal level

We must keep in mind that determinants within the distal level also interact with each other, adding complexity to our model. Some examples of important intralevel associations at this level are given below:

- As already mentioned, the globalisation-induced risk of conflict is often mediated by changes in other factors at the distal level stressing the importance of intra-level relationships at this level. For instance, increased global trade in arms, changes in economic inequity, inadequate policies and environmental degradation have all been associated with globalisation as well as with the risk of conflict (e.g., Zwi et al. (2002)). On the other hand, increased trade can reduce the risks of conflicts by increasing the interdependence among societies (Scholte, 2000). Conflicts themselves can, in turn, have profound impacts on other distal factors like economic growth, trade, social interactions, or the implementation of adequate policies.

- Changes in health-related policy, of course, have the potential to influence many distal health determinants. For example, food safety policies are gaining importance as a global health issue and can have profound implications for food trade. These policies aim to harmonise food safety measures in order to ease trade between countries and to protect consumer health (Miyagishima \& Kaferstein, 1998). On the other hand, new food safety regulations in developed countries have sometimes far reaching implications for exporters in developing countries as they face difficulties in 
meeting the increasingly stringent regulations imposed by the developed world and the countries that are not able to meet these new regulations are at risk of being excluded from global trade (Laurian, 2001).

- Adverse changes in the provision of ecosystem services can result in increasing migration (environmental refugees), growing risks of conflicts or compromised economic development.

- Economic development without regard for environmental concerns can result in environmental degradation with negative effects on the provision of ecosystem goods and services. For example, food trade often develops at the expense of the environment (FAO, 1996). Economic development without regard for the poor or for other social concerns can result in adverse effects on, for example, economic and social equity.

- Increasing (technological) knowledge can result in the mitigation of adverse effects on the provision of ecosystem goods and services (e.g., due to the widespread implementation of clean technology) or can boost economic development.

\subsubsection{Globalisation and proximal health determinants}

Figure 4.4 shows that the impact of globalisation on each proximal health determinant is mediated by changes in several distal factors (Figure 4.4; arrows 5-12). The most important relationships will be discussed in more detail below. It is important to note that health policies and health-related policies can have an influence on all proximal factors (Figure 4.4 ; arrow 5).

\section{Health services}

Health services are increasingly influenced by globalisation-induced changes in health care policy (Figure 4.4; arrow 5), economic development and trade (Figure 4.4: arrow 6), and knowledge (Figure 4.4; arrow 7), but also by migration (3: arrow 7). Although the WHO aims to assist governments to strengthen health services, government involvement in health care policies has been decreasing and, subsequently, medical institutions are increasingly confronted with the neoliberal economic model. Some argue that this will increase efficiency, while others are concerned that health is increasingly perceived as a private good leaving the law of the market to determine whose health is profitable for investment and whose health is not. According to Collins (2003) populations of transitional economies are no longer protected by a centralised health sector that provides universal access to everyone, and some groups are even denied the most basic medical services.

The privatisation of, and increasing trade in health services, can obviously have profound implications for the provision of proper health care. Although some argue that global market-based health will improve consumer choice, others 
point out long-term dangers such as the entrenchment of a two-tier health system, the movement of health professionals from the public sector to the private sector, inequitable access to health care and the undermining of national health (Hong, 2000; Walt, 2000). The illegal trading of drugs and the provision of access to controlled drugs via the Internet are also potential health risks (Lee \& Collin, 2001). Globalisation may also produce a potentially serious 'braindrain' if it fosters the migration of health-care professionals from developing to developed regions (Pang et al., 2002).

However, increased economic growth is generally believed to enhance improvements in health care. Increased (technological) knowledge resulting from the diffusion of information can further improve the treatment and prevention of all kinds of illnesses and diseases.

\section{Social environment}

The central mechanism that links personal affiliations to health is 'social support,' the transfer from one person to another of instrumental, emotional and informational assistance (House et al., 1988). Social networks and social integration are closely related to social support (Berkman et al., 2000) and, as a result, globalisation-induced changes in social cohesion, integration and interaction can influence the degree of social support in a population (Figure 4.4; arrow 9). This link is, for example, demonstrated by Reeves (2000), who discussed that social interactions through the Internet influenced the coping ability of HIV-positive individuals through promoting empowerment, augmenting social support and facilitating helping others. Alternatively, social exclusion is negatively associated with social support.

Another important factor in the social environment is violence, which often is the result of the complex interplay of many factors (Figure 4.4; arrows 5, 8 and 9). The WHO (2002c) argues that globalisation gives rise to obstacles as well as benefits for violence prevention. It induces changes in protective factors like social cohesion, knowledge and education levels, and global prevention activities. On the other hand, it also influences important risk factors associated with violence such as income inequality, collective conflict, and trade in alcohol, drugs or firearms.

\section{Lifestyle}

Due to the widespread flow of people, information and ideas, lifestyles also spread throughout the world. It is already widely acknowledged and demonstrated that several modern behavioural factors such as an unhealthy diet, physical inactivity, smoking, alcohol misuse and the use of illicit drugs are having a profound impact on human health (Beaglehole \& Yach, 2003; WHO, 1999, 2001b, 2002a). Individuals respond to the range of healthy as well as unhealthy lifestyle options and choices available in a community (Murray \& Smith, 2001), which are in turn determined by global trade (Figure 4.4; arrow 8), 
economic development (Figure 4.4; arrow 8) and social interactions (Figure 4.4; arrow 9).

Although the major chronic diseases are not transmittable via an infectious agent, the behaviours that predispose to these diseases can be communicated by advertising, product marketing and social interactions (Marks \& McQueen, 2001). Global trade and marketing developments drive, for example, the nutrition transition towards diets with high proportions of salt, saturated fat and sugars (Beaglehole \& Yach, 2003; Murray \& Smith, 2001). Another example is the worldwide spread of tobacco consumption as transnational tobacco companies take advantage of the potential for growth in developing countries (Beaglehole \& Yach, 2003; Cunningham, 1996; Lee et al., 2008; McDaniel et al., 2008) (Box 4.7). Additionally, the scale of cigarette smuggling poses a considerable global threat to the efforts to control tobacco consumption (Lee \& Collin, 2001). Illegal trade in illicit drugs poses similar problems. At the same time, the alcohol industry is almost as globalised as the tobacco industry (Jernigan, 1997).

However, health education can play a role in promoting healthy lifestyles by improving an individual's knowledge about the health effects of different lifestyle options (Figure 4.4; arrow 9). Besides health education, (global) policies can also directly discourage unhealthy behaviour by means of economic incentives (e.g., charging excise on tobacco) or other legislation (Figure 4.4; arrow 5). An effective implementation of the WHO's Framework Convention on Tobacco Control (WHO FCTC) (WHO, 2003c) is expected to have profound implications on tobacco related-policies and, hopefully, tobacco use.

Box 4.7: Globalisation and lifestyle: the spread of the 'brown-plaque'.

Cigarette consumption varies considerably among countries around the world and, in general, wealthier countries show a higher consumption rate than developing countries. However, while a decline in cigarette consumption has been observed in the developed countries, the annual consumption rate in less developed countries increased. This increase can be attributed to 'aggressive marketing', the absence of regulations and educational programs, and rising incomes (Cunningham, 1996; McDaniel et al., 2008). These past decades transnational tobacco companies have moved into Latin America, Asia, the former communist countries of central and eastern Europe, China and Vietnam. In less developed countries, advertisements portray smoking as part of the desirable Western lifestyle, and many people have little knowledge about its health effects (Cunningham, 1996). A study by Ezzati and Lopez (2001) estimated that in 20002.41 million people died of smoking in developing countries and 2.43 million in rich nations. Besides the direct health effects of smoking, tobacco consumption also indirectly affects health; when it comes to choosing how to spend family income, tobacco competes with food in some countries. In addition, the increasing consumption of tobacco competes with the growing of food, because the land used for the production of tobacco is often also suitable for other crops. Additionally, tobacco consumption is also an important global environmental issue as it contributes to deforestation (Cunningham, 1996). 
Physical living environment: infectious diseases pathogens

The spread of infectious diseases is probably one of the most mentioned health effects of globalisation and past disease outbreaks have been linked to factors that are related to the globalisation process (Table 4.5). The SARS outbreak demonstrated the potential of new infectious diseases to spread rapidly in today's world. The combination of movement of goods (Figure 4.4; arrow 10) and people (Figure 4.4; arrow 11), and profound changes affecting ecosystem goods and services (Figure 4.4; arrow 12) all contribute to increased risk of disease spread (Newcomb, 2003). For example, the globalisation of food production, trade and consumption has been associated with the increased spread and transmission of food born diseases (Lee, 1999b; Newcomb, 2003) (Hodges \& Kimball, 2005). Diseases like HIV/AIDS or hepatitis B can also spread through trade in infected biological products (e.g., blood) (Lee \& Collin, 2001).

Table 4.5: Globalisation and infectious disease emergence: examples (based on Newcomb (2003)).

$\begin{array}{ll}\text { Disease } & \begin{array}{l}\text { Factors believed to be associated with infectious disease emergence } \\ \text { as well as with globalisation } \\ \text { Possibly introduced from Asia by ships. } \\ \text { Cholera }\end{array} \\ \begin{array}{ll}\text { Disseminated by human travel. } \\ \text { Foot and Mouth disease }\end{array} & \begin{array}{l}\text { Global trade and transport facilitated movement of highly } \\ \text { contagious viral agent. }\end{array} \\ \text { (Taiwan 1997- UK 2001) } & \begin{array}{l}\text { Disseminated by human travel or human transport of infected birds } \\ \text { West Nile Virus }\end{array} \\ \text { (USA 1991) } & \begin{array}{l}\text { Land use factors contributing to the loss of avian diversity. } \\ \text { Disseminated by human travel. }\end{array}\end{array}$

(2003)

The global diffusion of knowledge and new technologies will improve the surveillance of infectious diseases and monitoring of antibiotic resistance (Feachem, 2001; Lee, 1999a) (Figure 4.4; arrow 11). Globalisation potentially increases the speed of responses in some cases. Wilson (1995) states that responding to disease emergence requires a global perspective- both conceptually and geographically- as the current global situation favours the outbreak and rapid spread of infectious disease. As a result, the policies and actions undertaken by the WHO are becoming increasingly important in controlling infectious diseases at a global level (Figure 4.4; arrow 5). For instance, the WHO played a critical role in controlling SARS by means of global alerts, geographically specific travel advisories and monitoring (Fidler, 2004). 
Food

Food trade has become an increasingly important factor with regard to food security worldwide (Figure 4.4; arrow 10). At present, however, the developed countries still subsidise their agricultural sectors, and tariffs for agricultural products remain relatively high. Economic liberalisation policies are expected to have profound implications- both good and bad- on food trade and, subsequently, on food security. The Food and Agriculture Organization of the United Nations (FAO) $(1996,2003)$ argues that free trade could potentially create access to better and cheaper food supplies via food imports, and could stimulate more efficient use of the world's resources as well as the production of food in regions that are more suitable to do so. Free trade permits food consumption to grow faster than domestic food production in countries where there are constraints on increasing the latter. Accelerated economic growth can also contribute to food security. According to The State Of Food Insecurity in the World 2005 (FAO, 2005), accelerating the progress towards an open and more equitable trading system is one of the key elements in the worldwide reduction in hunger.

On the other hand, the FAO $(1996,2003)$ acknowledges that increasing dependence on food imports goes hand in hand with a higher vulnerability to shocks arising in global markets, which can affect import capacity and access to food imports. Many food-insecure countries are not able to earn enough with exporting goods to pay for needed food imports. There is an increasing concern about the adverse consequences of food trade resulting from current asymmetric imbalances in the global market (e.g., Lang (1996), Stevens (2003), Shiva (2004), and Smaller (2005)). At present, the developed countries still impose protectionist trade policies by, for example, subsidising their domestic agricultural sectors (allowing domestic producers to sell their products more cheaply then their foreign competitors) and imposing relatively high agricultural tariffs on imported goods (making them less competitive with domestic goods). Stevens (2003) argues that the protectionist policies of the OECD countries, together with stricter food standards, are expected to affect Africa's food security. Africa is being 'squeezed', as the price of cereal imports could increase, while the volume and price of agricultural exports could decline. Shiva (2004) states that agricultural trade liberalisation- facilitated by the policies and rules of the WTO, the WB and the IMF- has resulted in a declining access to food and an intensification of hunger in India. As a result, food security based on self-sufficiency is a recurring theme among developingcountry members of the WTO.

At the global level, there are increasing international efforts to achieve widespread food security (Figure 4.4; arrow 5). For instance, the right to adequate food is directly addressed in the 1966 International Covenant on Economic, Social and Cultural Rights. In 1996, the World Food Summit reaffirmed the right of everyone to have access to safe and nutritious food. In 
case of extreme food-insecurity and insufficient import capacity, food aid may be provided in order to supplement scarce food imports. Globalisation can also affect food security by enhancing knowledge of foreign nations about the need for food aid (Figure 4.4; arrow 11) (FAO, 1996).

Besides food trade, one can also deal with the mismatch between demand and supply by increasing food production in food-short regions. The globalisation process can increase food security by facilitating the worldwide implementation of better technologies and improved knowledge (e.g., irrigation technologies, research on genetically modified food) (Figure 4.4; arrow 11). At the same time, the natural resource base for food production is increasingly threatened by compromised ecosystem functioning (Figure 4.4; arrow 12). Finally conflicts are, of course, a threat to food security and it is expected that food security in subSaharan Africa, for example, will not increase without the establishment of political stability (Figure 4.4; arrow 11) (Meade et al., 2003).

\section{Water}

The effects of globalisation are also raising concerns over water security. The current globalisation process is accompanied by privatisation policies affecting the provision of water (Gleick, 2002) (Figure 4.4; arrow 5). Governments and international financial institutions promote privatisation, because they believe it will promote market competition and efficiency. However, others are less optimistic about the effects of privatisation. In fact, some cases show that prices and inequalities in access even rise (Olivera \& Lewis, 2004). Gleick (2002) argues that water, with vital importance socially, culturally, and ecologically, 'cannot be protected by purely market forces'. On a global scale, there are increasing efforts to set up global guidelines or policies with regard to fresh water (Figure 4.4; arrow 5), however, none of the international declarations and conference statements requires states to actual meet individual's water requirements (Gleick, 2000).

The virtual trade of water is also believed to be of increasing importance (Figure 4.4 ; arrow 10). The water that is used in the production process of a commodity is called the 'virtual water' contained in that commodity. Therefore, the increasing global trade of commodities is accompanied by increasing trade in virtual water. In the period 1995-1999, the global volume of crop-related international virtual water flows between nations is estimated at $695 \mathrm{Gm} 3$ per year (13\% of total water use for crop production) (Hoekstra \& Hung, 2005). In addition, the globalisation process can increase water security by facilitating the worldwide implementation of better technologies and improved knowledge (Figure 4.4; arrow 11). At the same time, the natural resource base is increasingly threatened as, for example, global climate change and deforestation profoundly affect our ecosystems ability to provide us with sufficient and adequate fresh water (Figure 4.4; arrow 12). 
Importance of intra-level relationships at the proximal level

We must keep in mind that determinants within the proximal level also interact with each other, and some examples of important intralevel associations at this level are given below:

- Aspects of lifestyle can be important factors in the exposure to the physical environment. For example, the actual exposure to infectious disease pathogens in the environment is, to a large extent, determined by lifestyle factors such as, unhygienic practices.

- The social environment can reinforce unhealthy behaviours via social influence (NPHP, 2001).

- Water and food are two factors at the proximal level that are strongly interrelated; food production is heavily reliant on the availability of sufficient water, and often competes with other water uses such as sanitation or drinking.

\subsubsection{Discussion of conceptual framework}

Despite some empirical research efforts indicating the link between the globalisation process and specific health impacts, the present weakness in empirical evidence on the multiple links between globalisation and health is still a problem (Lee \& Collin, 2001). The framework provides valuable insights in how to organise the various factors involved in health effects resulting from globalisation. We claim that our approach has several beneficial characteristics. First, it is embedded in a holistic approach towards globalisation; we perceive globalisation as an overarching process in which simultaneously many different processes take place in many societal domains. In addition, the conceptual framework is embedded in a holistic approach towards population health. As a result, our model explicitly visualises that globalisation affects the institutional, economic, social-cultural and ecological determinants of population health and that the globalisation process mainly operates at the contextual level, while influencing health through the more distal and proximal determinants.

Therefore, the conceptual framework could give a meaningful contribution to further (empirical) research by serving as a well-structured 'think-model' or 'concept map'. It clearly demonstrates that an interdisciplinary approach towards globalisation and health is required, which draws upon the knowledge from relevant fields such as, for example, medicine, epidemiology, sociology, political sciences, (health) education, environmental sciences and economics. In addition, the model is considered a useful tool to structure scenario studies on the health implications of the globalisation process (see Chapter 5). 


\section{Chapter 5}

\section{A FUTURE WITHOUT HEALTH?}

Based on:

- Martens, P., \& Huynen, M.M.T.E. (2003). A future without health? Health dimension in global scenario studies. Bulletin of the World Health Organization, 81, 896 - 901 .

- Huynen, M.M.T.E., \& Martens, P. (2006). Globalization and human health: toward scenarios for the 21st century. In: Interactions between global change and human health. Vatican: The Pontifical Academy of Sciences. 


\section{A FUTURE WITHOUT HEALTH?}

In light of the international commitment to sustainable development, exploring our future has become a matter of urgency and relevance. How will global development evolve? Are we on a sustainable pathway? Will we be able to sustain ourselves as well as our planet? Obviously, studying the future of our health should be a vital part of this. However, the integrated assessment of future health impacts has not been a central issue in the past decade of global scenario analysis.

Developing health scenarios can provide important insights into the diverse health effects of globalisation by describing a range of possible interactions, and their consequences. To provide examples of how future health can be incorporated in existing scenarios, we link the institutional, economic, socialcultural, and environmental developments described by existing scenario studies to a set of possible health futures.

\subsection{SELECTED SCENARIO STUDIES}

For this study, we have selected existing scenario studies using several relevant criteria. The multiple drivers of changes in population health and long chains of causation require that relevant scenarios integrate across institutional, economic, social-cultural, and environmental dimensions. Since our study is concerned with long-term changes of the health status of the world's population, a long-range time horizon is required, preferably several decades. The scenarios should also be global to match the scope of the assessment, but regional disaggregation (at least between developed and developing countries) would be useful, since the analysis of future health transitions will be conducted at this scale. We searched for scenario studies that met these criteria using scientific literature, the Internet and the following two CD-ROMs: Future Trends 6: an information base for scanning the future (OECD, 2000a) and 2001 State of the Future (American Council for the United Nations University, 2001). With these criteria in mind - integration, long-range outlook, and global/regional scope - we considered nine scenario studies (with a total of 35 scenarios) since 1995. Table 5.1 gives a short description of each of the selected scenarios.

The scenarios by the Global Scenario Group (Gallopin et al., 1997)

The Global Scenario Group (GSG) was established to engage a diverse group of development professionals in a long-term commitment to examine the requirements for sustainability. The aim was to illuminate the character of the current global system, the dynamics driving it forward, and the spectrum of possible futures states and pathways. First, the study defined three broad 
classes of storylines, which they call Conventional Worlds, Barbarisation, and Great Transitions distinguished by, respectively, essential continuity with current patterns, fundamental but undesirable social change, and fundamental and favourable social transformation. Subsequently, two variants for each class were formulated, resulting in a total of six scenarios. Current driving forces (global political and economic change, structural inequity and persistent poverty, growing population, environmental problems, and technological innovation) are the common point of departure for all scenarios.

Millennium Project scenarios by the United Nations University (Glenn \& Gordon, 1998)

The Millennium Project of the American Council for the United Nations University is 'a global participatory futures research think-tank of futurists, scholars, business planners, and policy makers who work for international organizations, governments, corporations, NGOs and universities'. Scenario analysis is one of the principle components of the Millennium Project research agenda. As part of the project, a set of global exploratory scenarios looking out to 2050 have been developed. The drafting of the scenarios commenced with the choice of the principle independent dimension (axes) that seemed to force the worlds under examination to differ. This selection procedure was based on an informal inquiry that was conducted via e-mail and the project's home page. The selected axes of the exploratory scenario's are: 1) degree of globalisation (from free trade to isolationism), 2) communication technology (from vibrant to stagnant) 3) threats to global security and/or quality of life (from high to low) and 4) government participation in society (from high involvement to little or laisser-faire). The following four scenarios were selected for further development: Cybertopia, Rich Get Richer, Trading Places, Passive Mean World. These were used to provide the backdrop for the choice of the values of the exogenous variables in the selected model. As a result, the model output was consistent with the scenarios and provided quantitative estimates of indicators that were then incorporated within the scenario.

Global Scenarios 2000-2050 by the World Business Council for Sustainable Development (WBCSD, 1998)

The World Business Council for Sustainable Development (WBCSD) launched, on behalf of its partner companies, an effort to formulate a set of global scenarios to explore possible responses to the challenge of sustainable development. In particular, the underlying question was how businesses can respond to these challenges. A large group of multinational corporations participated in this project, which was led by a core team of experts from WBCSD, Shell International, and the Global Business Network. The scenarios aimed to stimulate broad discussion on the challenges of sustainable 
development for business and to provide a platform for more focused industry and corporate scenarios. The developers took a wide range of views so as to construct a basis for creative thinking. The scenario development was primarily based on three pre-determined elements or driving forces: the new, the many and the connected. The 'new' has to do with social and technological innovations and with the economy in which more and more new countries, new businesses etc. are involved. The 'many' deals with population growth and the 'connected' refers to the increasing connection among humans, and between humans and the environment. Subsequently, the set of plausible storylines was structured, relevant interconnections identified, and scenario logics defined. The resulting scenarios are: FROG!(First Raise Our Growth!), GEOpolity and Jazz.

Which World by Hammond (1998)

The work by Hammond builds on the work of the Global Scenario Group. The study aims to design a set of global scenarios that span many different ways of looking at the world. Hence, three scenarios were constructed to frame sharply contrasting images of the future, although Hammond recognises that in reality the world in 2050 is likely to contain elements of all three scenarios. The scenarios are also intended to be clearly within the realm of the plausible; therefore, they are based on existing trends. The names he has given to the scenarios are: Market World, Fortress World, and Transformed World. The scenarios are written from the perspective of 2050 , so it is a story about what happened in the last half century.

Global trends 2015 by the National Intelligence Council (NIC, 2000)

In 1999-2000, the National Intelligence Council (NIC) developed a set of global scenarios in close collaboration with U.S. Government specialists and nongovernment experts (e.g., academia, private sector) in a wide range of fields. The aim was to stimulate U.S. policymakers to think 'beyond their inboxes'. The resulting report builds on the outcomes of two workshops. The first workshop identified major factors and events that would drive global change, focusing on demography, natural resources, science and technology, the global economy, governance, social and cultural identities, and conflict. The second workshop developed four alternative global futures in which these drivers would interact in different ways through 2015. Each scenario was intended to construct a plausible, policy-relevant story of how this future might evolve: highlighting key uncertainties, discontinuities, and unlikely or 'wild card' events, and identifying important policy and intelligence challenges. The four scenarios (Inclusive Globalisation, Pernicious Globalisation, Regional Competition, PostPolar World) can be grouped in two pairs: the first pair contrasting the 'positive' and 'negative' effects of globalisation; the second pair contrasting 
intensely competitive but not conflictual regionalism, and the descent into regional military conflict.

Special Report on Emission Scenarios (SRES) by the International Panel on Climate Change (IPCC, 2000)

The most recent scenario effort of the Intergovernmental Panel on Climate Change (IPCC) was developed, via a broad consultative process, for estimating emissions of greenhouse gases over the coming century, taking into account input and perspectives from a wide, interdisciplinary research community. The resulting Special Report on Emission Scenarios (SRES) explores the global and regional dynamics that may result from changes regarding population, economy, technology, energy use, and agriculture (land use). The scenarios are intended to exclude catastrophic futures. The distinction between classes of scenarios was broadly structured by defining them ex ante along two dimensions The first dimension relates to the extent both of economic convergence and of social and cultural interactions across regions; the second has to do with the balance between economic objectives and environmental and equity objectives. This resulted in the creation of four scenario 'families' or 'clusters'. Whereas the ' $\mathrm{A}$ ' storylines (A1 and A2) emphasise economic development and leave only a subsidiary role for environmental and social concerns, the ' $\mathrm{B}$ ' storylines (B1 and B2) reverse these priorities. The ' 1 ' storylines (A1 and B1) emphasises successful economic convergence and social and cultural interaction across regions, while the ' 2 ' storylines (A2 and B2) focus on diverse regional developments.

World Water Scenarios by the World Water Council (Gallopin \& Rijsberman, 2000)

The approach in the World Water Vision project focused on developing qualitative scenarios to allow incorporation of the many social, economic, environmental and cultural factors that shape the water future. Models were then used to analyse the consistency and coherence of the qualitative scenarios, explore some of the consequences, and fill some of the gaps. The scenarios evolved in four rounds of development, discussion, feedback and subsequent improvement- with interactions among scenario developers, modellers, and groups working on visions for sectors and regions. The main forces affecting the global water scenarios are economic development, demographic developments, technological change, social trends and environmental quality. The three global scenarios that were developed are 'Business as usual' (BAU), 'Technology, economics, and private sector (TEC)' and 'Values and lifestyles (VAL)'. 
Table 5.1: Selected scenario studies.

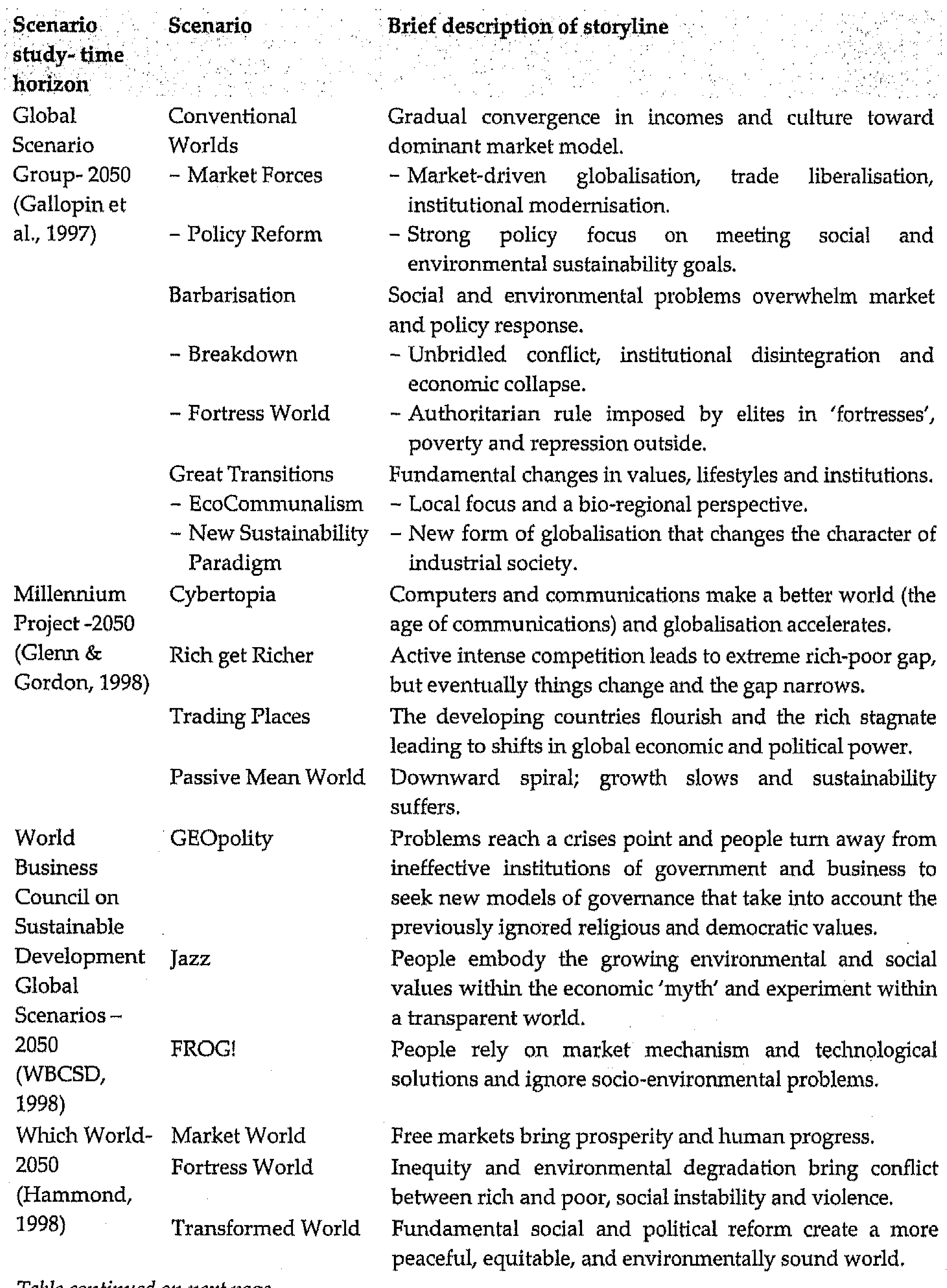

Table continued on next page. 
Table 5.1 continued.

$\begin{array}{ll}\begin{array}{l}\text { Scenario-study } \\ \text { (time horizon) }\end{array} & \text { Scenario } \\ \text { Global Trends- } & \text { Inclusive } \\ 2015 & \begin{array}{l}\text { Globalisation } \\ \text { Pernicious } \\ \text { Globalisation } \\ \text { Regional } \\ \text { Competition } \\ \text { Post-Polar World }\end{array} \\ & \text { A1 } \\ \begin{array}{l}\text { Special Report } \\ \text { on Emission } \\ \text { Scenarios } \\ \text { (SRES)-2100 }\end{array} & \text { A2 } \\ \text { (IPCC, 2000) } & \text { B1 }\end{array}$

B2

World Water

Scenarios- 2025

Business-as-Usual

(BAU)

Technology,

(Gallopin \&

Rijsberman,

2000)

Global environmental outlook

(GEO) 3- 2032

(UNEP, 2002) Ecosystem Assessment (MA) scenarios2050

(Carpenter et al., 2005)

Sector (TEC) (VAL)

Markets First

Policy First

Security First

Sustainability First

Adapting Mosaic

\section{Brief description of storyline}

Positive effects of globalisation and widespread global cooperation.

Globalisation's negative effects promote extensive dislocation and conflict.

Globalisation's negative effects spur intensely competitive but not conflictual regionalism.

Globalisation's negative effects spux regionalisms characterised by descent into regional military conflict.

Rapid market-driven growth, with convergence in incomes and culture.

Self-reliance and preservation of local identities; fragmented economic and technological development.

Convergent world with rapid changes in economic structures and emphasis on global solutions to sustainability.

Local solutions to economic, social, and environmental sustainability.

Current water policies continue, high inequity.

Market-based mechanisms, better technology.

Economics \& Private

Values and Lifestyles Less water-intensive activities, ecological preservation.

A world in which market driven developments converge on the values/expectations that prevail in industrial countries.

Strong actions are undertaken by governments in an attempt to reach specific goals.

A world of great disparities, where inequality and conflict prevail, brought about by socio-economic and environmental stresses.

A new development paradigm emerges in response to the challenge of sustainability, supported by new, more equitable values and institutions.

Global Orchestration Globalised, with emphasis on economic growth and public goods. Global economic and social approach to sustainability.

Order from Strength Regionalised, with emphasis on national security and economic growth. Protection through boundaries.

Regionalised, with emphasis on local adaptation and flexible governance. Local and regional approach to sustainability.

TechnoGarden
Globalised, with emphasis on green technology. Potential role of technology in providing and improving ecosystem services. 
Global Environmental Outlook (GEO3) scenarios by the United Nations Environmental Program (UNEP, 2002)

As part of its third Global Environment Outlook (GEO3), the United Nations Environment Programme (UNEP) developed a set of four alternative scenarios depicting developments for the 2002-2032 period. These scenarios were intended to stimulate thinking on different possible trends in the environment at the global and regional level. The GEO3 scenario exercise builds on the earlier work of the Global Scenario Group. It has been a highly participative programme involving experts from all around the world. A core scenario team of global and regional experts designed four global scenarios and, subsequently, regional teams elaborated these at the regional level. A number of models and analytical tools have been used to provide valuable underpinning of the storylines. The scenarios were refined through an iterative process involving the core scenario team, regional teams, modelling groups and external reviewers. The resulting scenarios- Markets First, Policy First, Security First and Sustainability First- describe possible futures based on an interpretation of prevailing driving forces (demography, economic development, human development, science and technology, governance, culture, environment).

The Millennium Ecosystem Assessment (MA) scenarios (Carpenter et al., 2005)

The Millennium Ecosystem Assessment (MA) scenarios address plausible future changes in ecosystems, in the supply and demand for ecosystem services, and in the consequent changes in human wellbeing. This scenario study builds on earlier scenarios and modelling efforts. Through a participatory process the following key concerns were identified; globalisation, leadership, poverty and inequality, technology, local flexibility and surprises. Framed in terms of context (increasingly globalised versus increasingly regionalised) and approaches (emphasis economic growth and the promotion of public goods versus emphasis on proactive ecosystem management), the MA scenarios are: Global Orchestration, Order from Strength, Adapting Mosaic and TechnoGarden. These scenarios were further developed in an iterative process of storyline development and modelling. According to the MA, the focus on alternative approaches to sustaining ecosystem services distinguishes these scenarios from previous global scenario exercises. In addition, they give an interesting overview of the circumstances under which particular scenario can branch into one of the other three scenarios. 


\subsection{THE HEALTH DIMENSION IN GLOBAL SCENARIO STUDIES}

In the selected scenarios, globalisation is often included as one of the important drivers of future change or even as a distinguishing factor between different storylines. But how well is the health dimension described in existing scenarios? We have evaluated the 'health-dimension' in the 35 scenarios described in the previous section; subsequently we have assessed whether the scenarios include other developments relevant for future health.

If one excludes the MA scenarios, Table 5.2 shows that only fourteen out of the 31 remaining scenarios give a reasonable description of future health. Eight scenarios completely neglect the health dimension. Nine scenarios describe only specific pressures/drivers with regard to human health or only certain aspects of health (e.g., mortality, water-related diseases). And even when a relative fair description of health is given, only four of these scenarios explicitly discuss future health as the integrated outcome of several social-cultural, economic and ecological developments. Health is largely missing in the Global Trends 2015 scenarios: three out of four scenarios do not mention it all. Remarkable is the fact that the Global Scenario Group developed six storylines of which two describe health in an integrated way and three do not mention anything at all about health. Additionally, the Millennium Project's scenario study includes two scenarios (Cybertopia and Trading Places) in which contrasting health developments are described without further explanation; both describe improving health and increasing life expectancy, while there are also emerging diseases and anti-biotic resistant bacteria. Overall, these findings indicate that health is not consistently handled within these existing sets of scenarios.

Contrary to other past scenario exercises, exploring future well-being (material well-being, health, good social relations, security, and freedom and choice) was one of the initial goals of the MA. In their own words, the MA provides 'a first order attempt to assess future health' (Corvalan et al., 2005). The report explicitly acknowledges the multi-causality of human health. Due to lacking quantification techniques to explore future health, however, quantitative data is limited (e.g., per capita water availability, food production, malnutrition). Other aspects relevant for human health are more qualitatively explored by means of the four storylines. The MA-report 'Ecosystems and human well-being: health synthesis' (Corvalan et al., 2005) outlines the possible future changes in health resulting from developments in 'critical drivers and other relevant factors'. As Table 5.2 indicates, all MA-storylines describe health as a more or less integrated outcome of multiple developments. However, the MA primarily focuses on the health implications of changing ecosystem functioning (resulting in a imbalance regarding the detailed description of environmental developments on the one side and social and economic factors on the other side) and on the risk of emerging diseases. 
Table 5.2: The health dimension in global scenarios.

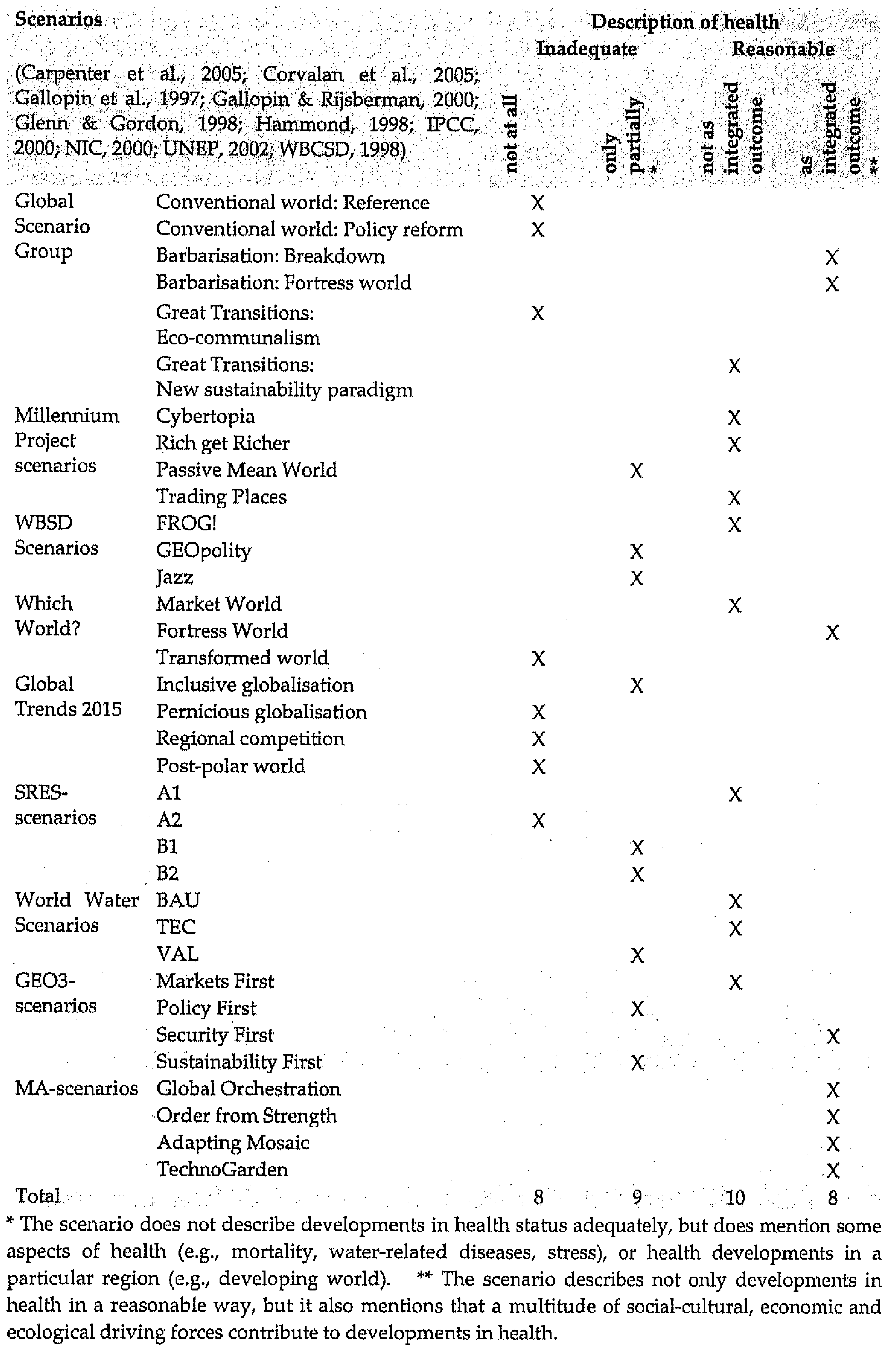


There are some inconsistencies in the MA-reports, which probably arise from the lack of a clear conceptualisation of population health and population health determination. It is mentioned that in three out of four scenarios global health will broadly improve for both developed and developing world (Carpenter et al., 2005). However, it is, for example, also stated that 'ecosystems services are indispensable to the well-being of people throughout the world' and 'under the MA scenarios, an increasing number of people may be unable to replace satisfactory, or escape from, the effects of depleted ecosystem services' (Corvalan et al., 2005). Also, in the Global Orchestration scenario, for example, there is an increasing risk of emerging infectious disease, ecological shocks, environmental pollution, and unhealthy lifestyles. Hence, the finding that health improvements are largest in this scenario is primary based on the (implicit) assumption that other developments (e.g., income growth, education, global governance and improved health services) are able to offset all these negative influences. With regard to the same scenario, one MA-report (Carpenter et al., 2005) claims that there is a slow response to climate change, while a second report (Corvalan et al., 2005) states that the supranational institutions in this scenario are well-placed to deal with global environmental problems such as climate change.

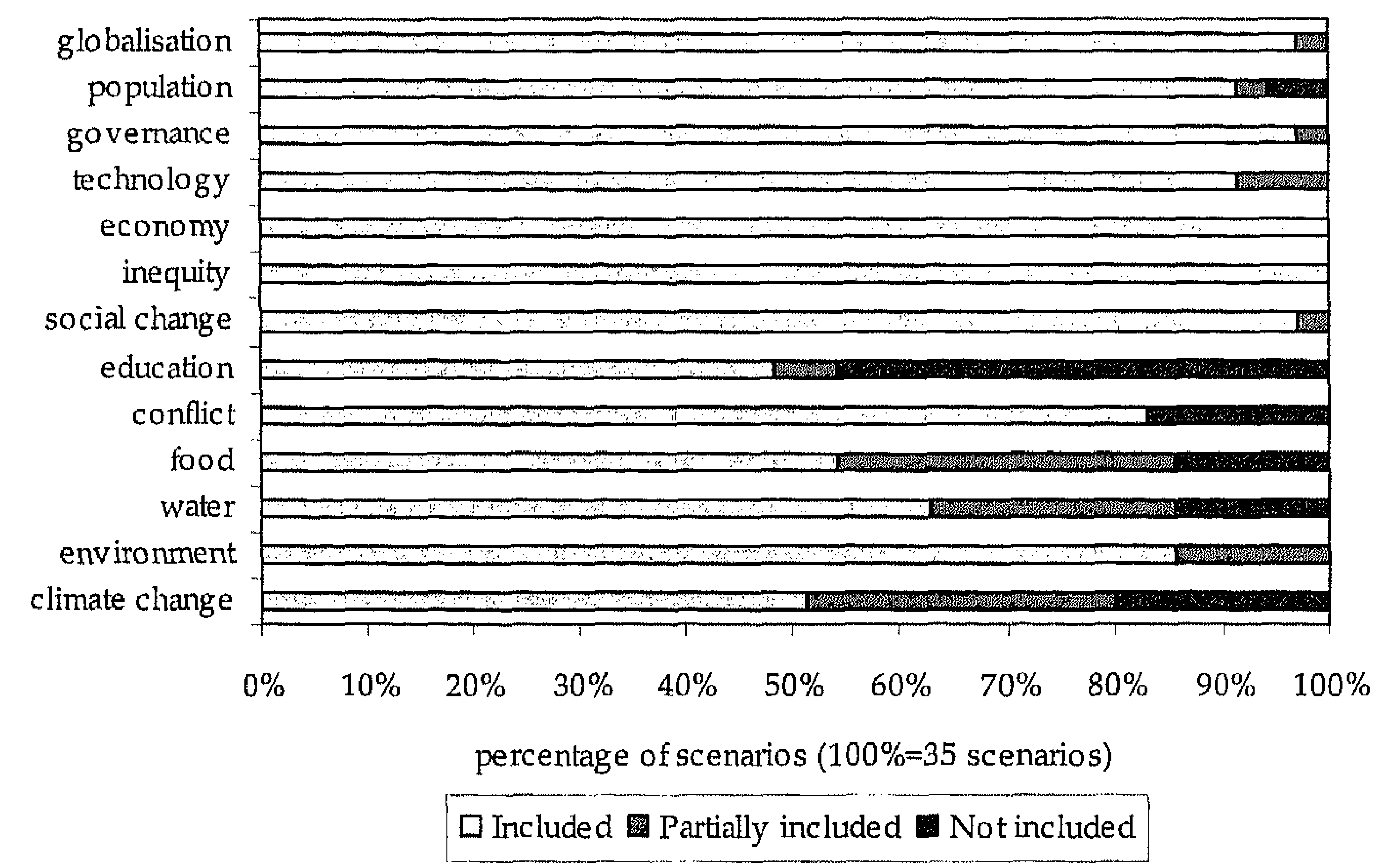

Figure 5.1: Descriptions of future developments in selected scenarios.

Note: This analysis is based on the original studies (Carpenter et al., 2005; Corvalan et al., 2005; Gallopin et al., 1997; Gallopin \& Rijsberman, 2000; Glenn \& Gordon, 1998; Hammond, 1998; IPCC, 2000; NIC, 2000; UNEP, 2002; WBCSD, 1998). Any further explorations made using these scenarios are excluded from this exercise. For example, the SRES-storylines do not mention any climate change developments, but they are widely used for further (model)calculations/explorations regarding future climate change. 
Looking at other developments that might influence our future health, Figure 5.1 shows that 'globalisation', 'population', 'inequity', 'conflict', 'social change', 'environment', 'economy', 'technology' and 'governance' are very well described in the majority of the selected scenarios. Less well described are 'food', 'water', 'climate change' and 'education'.

To conclude, a mere $23 \%$ of the selected scenarios describe health adequately and in an integrated way. Health is not consistently handled within the current sets of global scenarios. However, looking at other developments that possibly affect our future health, we see that many are well addressed in most scenarios. It, therefore, would have been possible to describe the future developments in health as an outcome of these multiple drivers and pressures.

\subsection{LINKING SCENARIOS TO HEALTH}

So what health future lies ahead? We explore this question by linking the institutional, social-cultural, economic, and environmental developments described in three sets of scenarios- SRES (IPCC, 2000), GEO3 (UNEP, 2002), and MA (Carpenter et al., 2005; Corvalan et al., 2005)- to a set of possible, but imaginary, health futures.

\subsubsection{Possible health futures}

Although the future of human health cannot be predicted, there are patterns of change and signs that can be anticipated. Below, a set of possible, but imaginary, health futures are sketched, building on past and current transition 'stages'1 (Huynen \& Martens, 2006). These health futures are based on existing views in current literature and possible 'early signs' observed within our society. They could follow from stages in the epidemiological transition as we have seen in the past and are facing at present. Additionally, they are not sharply delineated- there is always a continuum. There is also the possibility that economic, political, social, or environmental crises will cause the process of transition to stagnate, or to go into reverse.

Age of emerging infectious diseases

Current outbreaks of SARS and other (re)emerging diseases remind us that sudden disease emergence is a permanent part of our world and should be anticipated upon (Newcomb, 2003). It is recognised that communicable diseases are possible threats to the future of mankind; it would be 'catastrophic as viruses should evolve that exhibited abilities as those of HIV, and could be air-

\footnotetext{
${ }^{1}$ See Chapter 3 for more information on the epidemiological transition.
} 
born or propagated by casual contact' (Omran, 1998). According to Olshansky et al. (1998), for example, the next stage in the health transition could possibly be characterised by emerging disease outbreaks.

In this picture of future health, the 'age of emerging infectious diseases' (Martens, 2002), the emergence of new infectious diseases or the re-emergence of 'old' ones will have a significant impact on health. A number of factors will influence this development: travel and trade, microbiological resistance, human behaviour, breakdowns in health systems, and increased pressure on the environment (Barrett et al., 1998). Social, political and economic factors that cause the movement of people will increase contact between people and microbes; environmental changes caused by human activity (e.g., dam and road building, deforestation, irrigation, and climate change) will all contribute to the further spread of disease. The overuse of antibiotics and insecticides, combined with inadequate or deteriorating public health infrastructures will hamper or delay responses to increasing disease threats. As a result, infectious diseases will increase drastically, and life expectancy will fall (as is currently the case in many developing countries due to the AIDS pandemic). Ill health will lead to lower levels of economic activity, and countries will be caught in a downward spiral of environmental degradation, depressed incomes and bad health. Control of infectious diseases will be hampered by political and financial obstacles, and by an inability to use existing technologies.

\section{Age of medical technology}

Economic development (and associated modernisation processes) and developments in (medical) technology and health care are believed the have played a major role in the past shifts in health patterns and risk factors (Omran, 1983, 1998). Vice versa, the shifts in health and disease patterns have influenced the organised response to the changing needs of the population, particularly regarding the provision of health services (Bobadilla \& Possas, 1993). In the developed world, for example, the emergence of chronic health problems and unhealthy lifestyles has already changed the main business of health systems dramatically. In the developing world, policies have concentrated on the widespread implementation of modern health care and development programmes.

An extension of these trends into the future could possibly be described as the 'age of medical technology' (Martens, 2002). Such a future is in line with Omran's (1998) futuristic stage called 'aspired quality of life with paradoxical longevity and persistent inequities'. There will be continued achievements in disease control, health promotion, and prolongation of life. To a large extent, increased health risks caused by, for example, unhealthy lifestyles and environmental changes will be offset by increased economic growth and technology improvements. Still, some health problems will, at least for a while, challenge the existing diagnostic and therapeutic abilities (just as with the 
evolution of HIV/AIDS). Additionally, longevity is a mixed-blessing with increasing chronic morbidity and mounting medical costs. There will also be continued socio-economic inequities. If there is no long-term, sustainable economic development, increased environmental pressure and social imbalance may eventually propel poor societies into the 'age of emerging infectious diseases'. On the other hand, if environmental and social resources are eventually balanced with economic growth, sustained health may be achieved in the long run.

\section{Age of sustained health}

The Earth Charter Initiative (Blaze Corcoran et al., 2005) is a good present-day example of a global movement promoting a global ethic for a sustainable world. It is based on the participation of thousands of organisations, groups, and individuals worldwide. The Earth Charter envisions a future characterised by a societal transformation towards sustainability, which in the document itself is called 'a change of mind and heart'.

From a health perspective, such a future can possibly be described as an 'age of sustained health' (Martens, 2002). Economic growth will stay within social and ecological limits. Combating the wide-range of risk factors, social participation, social justice and harmony with the environment play an important role in achieving physical, mental, spiritual and social well-being. Investments in social services will lead to a sharp reduction in lifestyle related diseases, and most environmentally related infectious diseases will be eradicated. Health policies will be designed to improve the health status of a population in such a way that the health of future generations is not compromised by, for example, the depletion of resources needed by future generations. Although there is only a minimal chance that infections will emerge, improved worldwide surveillance and monitoring systems will mean that any outbreak is properly dealt with. Due to the ageing of the world population, health systems will be well adjusted to an older population. Furthermore, disparities in health between rich and poor countries will eventually disappear. This picture of future health is comparable with Omran's (1998) vision of future health described as 'quality of life, equity, development, and social justice for all', which takes a holistic view of health in the context of human well-being and human rights.

Additional future stage for non-western countries: age of chronic diseases

For the developing countries, an additional possible health future can be distinguished. In this future, they experience a shift towards a state that resembles the present-day situation in the developed world: infectious diseases make way for chronic diseases in the elderly. This future is in line with Omran's (1971) original view on the epidemiological transition, assuming that the developing countries will eventually follow the same pathway as the developed world. For practical reasons, this additional fourth health future for the 
developing world is called the 'age of chronic diseases'. However, it is possible for current developing countries to skip the 'age of chronic diseases' and shift directly to the 'age of sustained health', 'age of medical technology' or 'age of emerging infectious diseases'.

\subsubsection{Future health in the SRES-scenarios and the GEO3-scenarios}

This section explores which future health images can evolve from the developments described in the four SRES-scenarios (IPCC, 2000) and the four GEO3-scenarios (UNEP, 2002). The projected picture of future health in each scenario evolves from (our interpretation of) the combination of the described developments in relevant health determinants.

Future health in SRES-A1

In the future of $A 1$, economic growth, technological developments and globalisation will play a central role. Global population peaks in mid-century and declines thereafter due to fast worldwide demographic transition. This scenario describes decreasing mortality and increasing life expectancy due to economic growth. There is an emphasis on the health needs of an ageing population. Although economic development will contribute to improvements in social conditions, the focus on economic growth may lead to the 'social exclusion' of some communities. Relative income disparities decrease, but absolute differences remain large. Additionally, income growth will put pressure on (global) resources and, thus, lead to ecological degradation. Market-based and technological approaches are the common response to environmental problems.

From a health perspective, we might see a divergence between the developed world and parts of the developing world. In developed countries, increasing wealth, technology, and improvements in healthcare will offset most of the emerging risks. At least in the short-to-medium term, material advances, allied with improving social conditions, will lead to gains in overall population health. As a result, the richest populations may experience particularly pronounced health improvements and they advance to the 'age of medical technology'. The poorer countries will experience economic growth and subsequent health improvements, leading to increased life expectancy and increasing prevalence of chronic diseases. But at the same time, the absolute income differences remain and the poorer countries will not be able to advance to the 'age of medical technology', because they do not have sufficient means to finance wide-scale use of newly developed technologies (despite the diffusion of technological knowledge). As a result, they are not able to achieve the same level of health care as the developed countries, and they experience more difficulties with averting the negative health impacts of increasing 
environmental pressures (e.g., water scarcity). Consequently, there may also be resurgence of old diseases and an increase in new infections. The developing world will likely experience an increase in both chronic and infectious diseases ('age of chronic disease'/'age of emerging infectious diseases').

Future health in SRES-A2

In the future of A2, health will largely be left to individual choice and less of an issue for public policy. Economic development is moderate compared to A1. The greatest economic growth will take place in the developed regions, and technological advances will benefit only rich countries, as there will be limited diffusion of knowledge. Developed countries will make increasing investments in better welfare. However, globally, the gains in health brought about by economic development and technology will be partly offset by environmental problems and the exacerbation of the rich-poor gap between and within countries. While most developed countries will be able to counteract part of the threat of emerging infectious diseases by increasing investment in public health and medical care (slowly advancing towards the 'age of medical technology'), the proportion of infectious diseases that contribute to the total burden of disease will increase due to population growth and ecological degradation combined with only moderate economic growth and 'leaner' governments. The situation will be fragile, and in some developed countries the risk of infectious disease may rise considerably, with the potential of falling back into the 'age of emerging infectious diseases'.

In developing countries, levels of health and welfare spending will either remain the same or decline. In poor countries, current barriers to the control of such major diseases as malaria are likely to persist, and the importance of adequate water and food supplies will increase as population growth is high and environmental degradation increases. This combination of limited economic resources, high population growth, and increasing pressures on the local and global environments will increase the prevalence of infectious diseases, leading to the 'age of emerging infectious diseases'.

Future health in SRES-B1

A central element in the B1 scenario is a high level of environmental and social consciousness, combined with a global approach to sustainable development. In the developed world, mortality declines and life expectancy increases due to improved social infrastructures and institutions, economic growth, dematerialisation, and investments focused on decreasing pressure on ecological systems via the sustained management of resources. An extensive welfare net will prevent poverty-based social exclusion. Although the population will age due to the fast worldwide demographic transition, healthcare systems will probably be well adjusted to an older population. 
Under this scenario, developed countries may well complete the transition towards the 'age of sustained health'.

Thanks to transfers of knowledge and technology, declining national debts, low population growth, increasing education levels, and decreasing social en environmental pressures, the developing world will experience some significant health improvements. Although some countries will arrive at the 'age of chronic diseases' (i.e., the stage at which the developed world finds itself today), the global approach toward sustainability will enable most of them to skip this stage and move toward the 'age of sustained health'.

Future health in SRES-B2

In the B2 scenario, there will be increased concern for environmental and social sustainability in a heterogeneous world. Governments will, therefore, find community- and policy-based solutions to environmental and health problems. Most governments will likely increase public spending, including that on public health. Environmentally aware citizens will exercise a growing influence on national and local policy. There will be a shift to regional and local decisionmaking, with a high priority being given to human welfare, equality, and environmental protection. Education and welfare programs will be widely pursued, reducing mortality and fertility. But in this differentiated world, social and environmental progress is relatively slow, and will vary across regions and countries. Increased expenditure on 'health' and 'environment' will be implemented first in richer countries and it will take time for developing countries to follow.

In this scenario, some countries will be more successful than others. Developing countries may experience an increase in life expectancy and chronic diseases (moving slowly to the 'age of chronic diseases'), and some may eventually also achieve some technological progress by themselves. However, due to the slow pace of change, the developing world will not be able to make any significant progress towards a true sustainable society within the given timeframe. For developed countries, the situation will be more robust than in A2; they will slowly start to advance towards the 'age of sustained health', possibly via the 'age of medical technology'. But the transition towards sustainability is far from complete, and whether they will ever be able to achieve sustained health beyond the timeframe of the scenario will probably depend on further health developments in current developing countries. For the developed and developing countries that are lagging behind the risk of emerging diseases increases. It is also important to note that there is a lack of global governance, which could cause difficulties in solving any global problems. If, for example, severe global environmental changes occur, the improvements in health will be affected, or may even be reversed. 
Future health in GEO3-Markets First

Markets First describes the continuation of economic growth and globalisation. Environmental and social issues are valued as important, but do not have the highest priority. Governments primarily rely on market-based and technological solutions. However, the technological innovation achieved cannot keep pace with the combination of economic development and population growth. As a result, the increase in social problems and environmental degradation continues. Human health is negatively affected by ongoing population growth (especially in the developing world), high migration pressures, regional conflicts (e.g., in Africa), the ongoing AIDS pandemic, pressures on food and water, losses in biodiversity, pollution and climate change. However, there are improvements in medical technology and health care. Although the developing world participates in the global market, inequity and poverty persist.

Due to growing environmental and social health pressures combined with serious economic troubles, developing countries will have a difficult time trying to reach the 'age of chronic diseases'. They will slowly become overwhelmed due to the accumulation of social, environmental and economic problems and gradually shift into the 'age of emerging infectious diseases'. The developed countries, on the other hand, will continue to go on as they are; using economic and technological means to avert negative health impact. They will advance to the 'age of medical technology'. But as the pressures on health continue to increase and the migration from South to North can spread infectious diseases, they must keep in mind that there is a considerable risk of falling into the 'age of emerging infectious diseases'.

Future health in GEO3-Policy First

In Policy First, sustainable development will become the corner stone of policy agendas. Unfortunately, this future is also characterised by slow progress and mixed results of policy measures. There are advances regarding education, reducing extreme poverty, environmental quality and the slowing down of population growth. However, the progress regarding food and water availability cannot keep pace with the increasing demand, especially in developing countries. Other problems also remain or even increase: inequity (although efforts are being made to lower foreign debts and stimulate development in developing countries), regional conflicts, and climate change. In this scenario, there is some progress towards sustainability, but a lot of work still has to be done. The scenario itself describes improvements in health regarding infant and child mortality.

In this future, the developed countries are in the process of shifting towards the 'age of sustained health', but within the timeframe of the scenario they have not reached the completion of this transition by far. Whether they will ever be able to achieve sustained health will strongly depend on the health developments in 
the current developing world. The developing countries will not be able to benefit as much from the described improvements as the developed world, as inequity still exists. They probably advance to the 'age of chronic diseases', but it is unclear whether they will ever be able to progress towards the 'age of sustained health'.

\section{Future health in GEO3-Security First}

The main characteristic of Security First is the enormous increase in the richpoor gap. In this 'future of inequity', the poor will inevitable be the first victims of the adverse effects of the numerous and growing pressures on public health like the increasing resource problems (e.g., food and water scarcity), environmental degradation, conflicts, migration, population growth, lack of education, inadequate healthcare, the continuing AIDS pandemic and climate change. The scenario describes the resurgence of old diseases and the emergence of new diseases. The relative slow technological progress only benefits the rich. In addition, social problems have no priority and the economy stagnates.

Society finds itself in a downward spiral and the poorest countries will not be able to advance to 'age of chronic diseases'. The social, environmental and economic pressures will lead them to the 'age of emerging infectious diseases' very rapidly. The rich, however, can avert the negative health impacts, at least in the short-to-medium term. They live separately from the poor in (metaphorical) fortresses, where they are (temporarily) protected against environmental and social problems and where they have access to proper health care and medical technology. So at first, the developed world will be able to maintain in the 'age of chronic diseases' or even advance to the 'age of medical technology'. But the situation for the rich is less robust than in Markets First; the proportion of communicable diseases that contribute to the total burden of disease will grow. The question remains how long it will take before the rich eventually shift completely into the 'age of emerging infectious diseases' as the social, environmental and economic pressures from the outside continue to increase.

Future health in GEO-Sustainability First

In Sustainability First, people embrace a new sustainability paradigm. Social issues (including health) and environmental quality have high priority, policy measures have strong results, and there is gradual economic growth within the limits of sustainable development. This scenario describes a successful transition towards sustainability resulting in great reductions in the pressures on public health; stabilisation of population at moderate levels, increasing education levels, reductions in conflicts and tensions, increasing environmental quality, sufficient water, and sufficient food. Also very important are the 
closing gap between rich and poor, and the deliberate efforts to reduce child mortality and to increase life expectancy.

In the future of Sustainability First, conditions become favourable in order to reach the 'age of sustained health', both for the developed countries as for the developing world. It is even possible that current developing countries skip the 'age of chronic diseases' and advance directly to the 'age of sustained health'.

\subsubsection{Future health in the MA-scenarios}

This section explores which future health images can evolve from the developments described in the four MA-scenarios (Carpenter et al., 2005; Corvalan et al., 2005). The projected picture of future health in each scenario evolves from (our interpretation of) the combination of the described developments in relevant health determinants. For this exercise, we make use of the conceptual framework developed in Chapter 4.

\section{Future health in Global Orchestration}

The Global Orchestration scenario unfolds an increase in globalisation with a focus on economic and social policies. Fair economic liberalisation and social equity have high priority. Fair trade expands, economies grow substantially, and the rich-poor gap declines. Nevertheless, some regions lag behind. Due to the global governance structures, global public health monitoring is put in place and the institutional capacity to respond to health threats- such as disease outbreaks- increases (but still remains lower in developing world). Global communication and mobility is high, and a diverse global society emerges. Social interactions increase through global travel and networks, social equity and investment in social capital. Democracy and cooperation result in declining tensions. There are high investments in education and human capital, combined with a global flow of knowledge and technology. The environment has less priority, as people rely on economic growth, technology and market mechanism to react to any mounting ecological problems. There is limited environmental research. This results in slow progress and mixed results regarding environmental management. Negative pressures on ecosystem functioning increase (e.g., due to global environmental change, and economic growth) and environmental security declines, resulting in a risk of ecological surprises.

These envisioned developments in the contextual and distal level of health determinants affect the multiple proximal determinants (Table 5.3). The quality of health services (e.g., primary care, laboratories, and hospitals) benefits from the wide availability of healthy technologies and economic development. There is improved access to health care, but some regions still lag behind. The reactive approach characterising this scenario could also spill into health services provision (i.e., prevention has less priority). Social relations improve, but some 
groups lag behind. Violence rates are expected to decrease. Unfortunately, global efforts to promote healthy lifestyles are rather unsuccessful. Despite the increasing pressures on food systems, food availability increases and malnutrition decreases, probably due to reduced poverty, technological developments and the focus on equality. Levels of (severe) water stress increase over today's level in many developing countries. Declining water quality might also pose some problems, especially in poor regions. People increasingly demand 'beautiful' surroundings, but long-term and hard-to-address environmental problems start to have an affect on the physical living environment, in particular affecting the poor. The risk of infectious disease emerges due to deteriorating ecosystems, travel and trade. If health risks are unexpected, control proves to be difficult, despite global response structures. It is important to note that the increasing (global) environmental problems possibly affect the economy in the long run.

The MA-assessment reports that health improvements are largest in this scenario. However, Table 5.3 shows that there are also multiple unhealthy developments in proximal determinants. Hence, the MA analysis is based on the (implicit) assumption that other developments (e.g., income growth, education, global governance, and improved health services) are able to offset these negative influences.

Table 5.3: Global Orchestration- developments in proximal health determinants.

\begin{tabular}{|c|c|c|}
\hline Proximal heatth & Developed regions & Developing regions \\
\hline Health services & $\begin{array}{l}\text { Improvements in quality and } \\
\text { access }\end{array}$ & $\begin{array}{l}\text { Strong improvements in quality } \\
\text { and access for most regions }\end{array}$ \\
\hline $\begin{array}{l}\text { Social environment } \\
\text { \& support }\end{array}$ & Improvements & Improvements for most regions \\
\hline Lifestyles & Increasingly unhealthy & Increasingly unhealthy \\
\hline Violence & Declines & Declines \\
\hline Water & $\begin{array}{l}\text { No severe water stress in OECD } \\
\text { countries }\end{array}$ & $\begin{array}{l}\text { Increasing water stress and water } \\
\text { quality declines }\end{array}$ \\
\hline Food & Improving availability & $\begin{array}{l}\text { Strongly improving availability } \\
\text { and declining malnutrition }\end{array}$ \\
\hline $\begin{array}{l}\text { Physical } \\
\text { environment }\end{array}$ & $\begin{array}{l}\text { Increasing pressures } \\
\text { In the longer run: risk of emerging } \\
\text { infectious diseases }\end{array}$ & $\begin{array}{l}\text { Increasing pressures with } \\
\text { disproportional impacts on the } \\
\text { poorest regions } \\
\text { Risk of emerging infectious } \\
\text { diseases }\end{array}$ \\
\hline
\end{tabular}




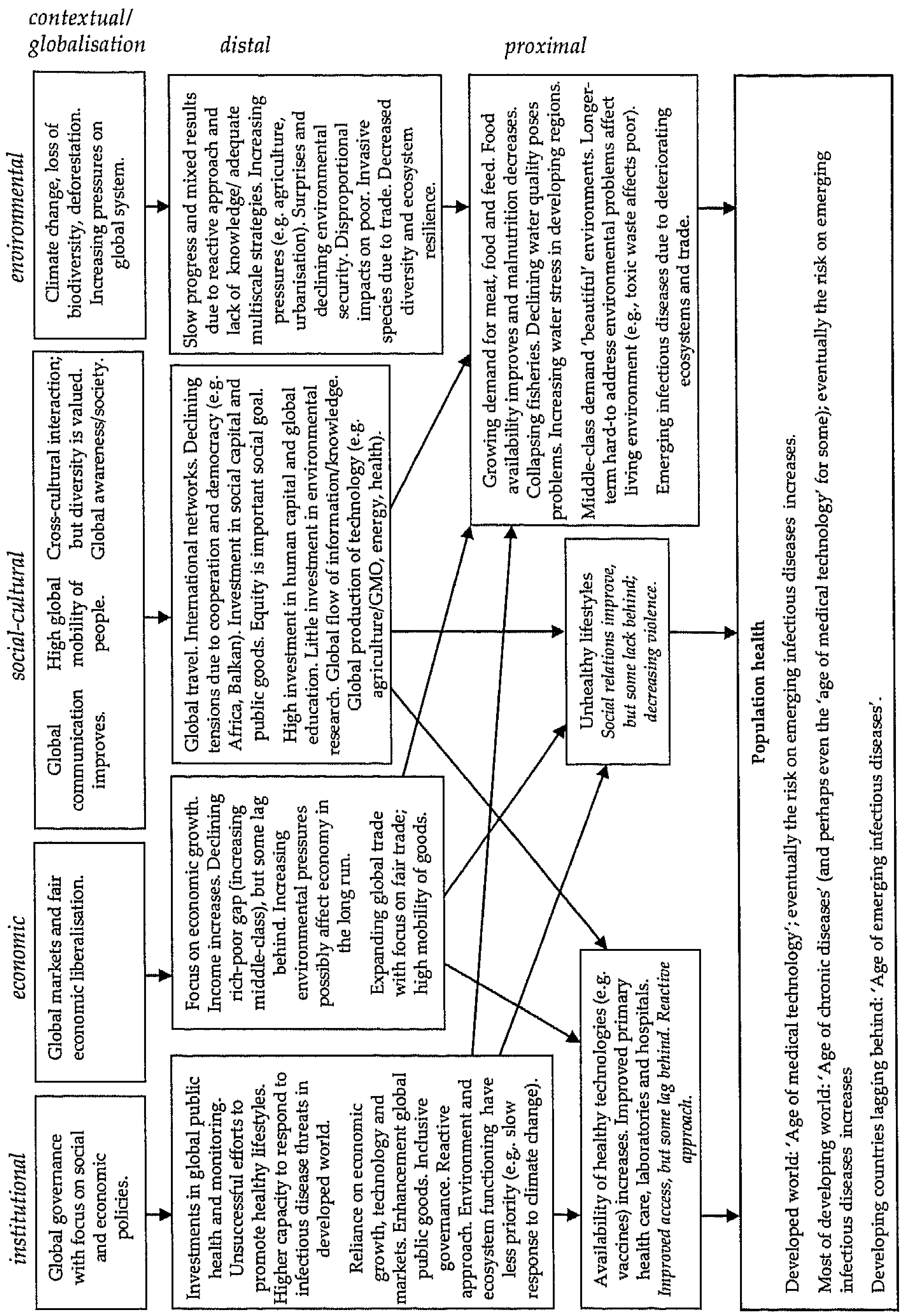

Figure 5.2: Future health in Global Orchestration.

Note: Developments that were not specifically mentioned in the MA-documents, but evolved from our interpretation of the combination of the described developments in other relevant factors, are indicated by italics. 
Based on Figure 5.2 and Table 5.3 we conclude that the developed world will probably advance into the 'age of medical technology'. Most of the developing world will see some great health improvements, shifting to the 'age of chronic diseases', and perhaps even into the 'age of medical technology'. Both developed and developing regions face, however, an increasing burden of unhealthy lifestyles and a coexisting risk of emerging infectious diseases. Additionally, the scenario also has two important drawbacks to keep in mind. First, not all developing countries will be able to benefit from these (health) improvements and the increasing risk of emerging disease will lead them on their way to the 'age of emerging infectious diseases'. Second, the growing environmental problems can have a negative impact on development in the long run, increasing the risk of shifting into the 'age of emerging infectious diseases' in other regions as well.

\section{Future health in Order from Strength}

Order from Strength is characterised by fragmentation and inward-looking national policies focussing on national security and protectionism. Economic liberalisation retreats and trade primarily takes place in regional trading blocks (but trade between poor countries is limited). Importantly, the power lies with the economic and military strong nations. Policymakers rely on economic growth and technological solutions, while fragmentation and narrow interests mask the global reality. The rich-poor gap increases rapidly and most developing countries are left behind (e.g., limited foreign aid). The Western countries face stagnating economies. There is only limited legal mobility (e.g., reduced travel, strict immigration policies), but migration pressures increase and, consequently, illegal migration within and between regions grows. There is only limited cross-cultural interaction. Due to this fragmentation, there is by no means a sense of a global society. Some global communication takes place, although the poor are excluded (e.g., digital divide). Moral character deteriorates. Safety nets for the poor collapse and social exclusion (within and between nations) increases. Due to high spending on security, there is little investment in human capital (e.g., education). Technological progress is slow and knowledge is not globally shared, as scientific exchange weakens. The inward-looking focus on security results in increasing tensions and a lack of trust between countries (e.g., conflicts over water). Conflicts prevail outside rich people's walls.

Regulations are in place dealing with disease outbreaks, but the underlying problems remain unaddressed. No long-term health solutions are being developed, and the prevention and cure of important disease is neglected. Learning about the environment has no priority. Environmental problems are only addressed with reactive policies focusing on securing resources. Global environmental problems increase significantly (e.g., biodiversity loss, deforestation, desertification and soil erosion). Climate change impacts affect 
both rich and poor. This future faces declining provision of ecosystem services (even to some elites), increasing ecosystem vulnerability and environmental collapse. Ecosystems are pushed to their limits and environmental protection is highly fragmented. Access to ecosystem functions becomes very uneven and developing countries are increasingly exploited. As a result, the poor face ecosystems below minimal levels. The elites/rich increasingly feel more and more pressure from 'outside' as well.

These envisioned developments in the contextual and distal level of health determinants affect the multiple proximal determinants (Table 5.4). The poor have limited access to health services and medicines. The development of healthy technology is limited as well, negatively affecting the health systems' quality. Due to the reactive approach characterising this future, prevention of diseases will probably not have a very high priority. Social relations deteriorate ('dog eat dog world'). Violence rates increase, especially in poor regions. Healthy lifestyles have less priority. Developing countries experience substantial problems with food availability and malnutrition due to lack of technology, declining social services and ecosystem deterioration (e.g., collapsing fisheries). Water stress rises in many regions and water quality declines (e.g., nutrient pollution). The poor are most at risk of environmental exposures (e.g., pollution, urban waste, natural disasters, resource scarcity). The rich minimise impact through technology, but wealthier countries continue to experience pressures from outside. Disease outbreaks emerge due to environmental problems and trade. New disease outbreaks become very common in poor regions.

Table 5.4: Order from Strength- developments in proximal health determinants.

Proximal health
determinants
Health services
Social environment
\& support
Lifestyles
Violence
Water
Food
Physical
environment

Developed regions
Available to the rich
Limited improvement in quality
Declining
Healthy lifestyle have no priority
Increasing tensions
Levels of water stress stabilise
Adequate availability
Negative impacts are partly offset,
but for how long?

Developing regions

Limited availability

Strongly declining

Healthy lifestyle have no priority

Increasing violence

Water stress significantly increases

Malnutrition increases

Strongly deteriorating living

environments

New diseases are common 


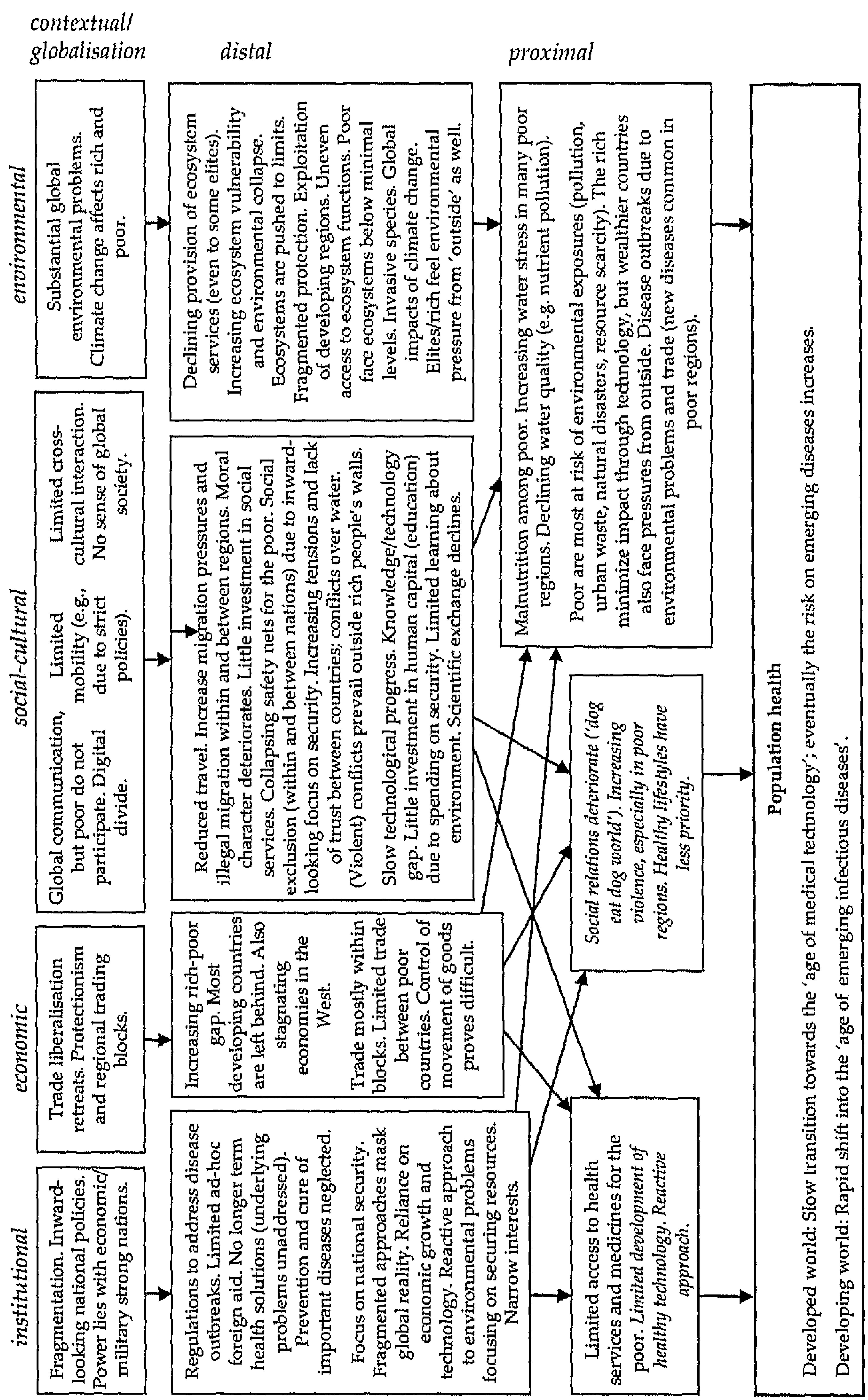

Figure 5.3: Future health in Order from Strength

Note: Developments that were not specifically mentioned in the MA-documents, but evolved from our interpretation of the combination of the described developments in other relevant factors, are indicated by italics. 
Based on Figure 5.3 and Table 5.4 we conclude that the developing countries are caught in a negative spiral of poverty, exclusion, lacking (health) services, violence, water and food scarcity, and environmental degradation. These detrimental developments will lead them to the 'age of emerging diseases' very rapidly. The developed countries will be able to offset some of the negative developments by focussing on national (environmental) security, at least for a while. However, technological development is slow and the rich countries will only advance towards the 'age of medical technology' at a very low pace. Unhealthy lifestyles and environmental pollution will increase chronic diseases, but eventually the rich will progressively experience the negative effects of, for example, (global) environmental change, water stress, violence, and a lacking global approach to address disease outbreaks. Eventually they will start to shift to the 'age of emerging infectious disease' as well.

\section{Future health in Adapting Mosaic}

In Adapting Mosaic, globalisation reverses and makes way for fragmented approaches to sustainability. The current division between developed and developing world changes ${ }^{2}$. Local and regional governance emphasises adaptation and flexible governance, and civic society plays an important role. Proactive policies aim at balancing natural, human and manufactured capital, but there is a diversion of management recourses and the heterogeneous policy measures yield mixed results. Global (health) policies are lacking. Despite the fragmentation, interregional communication levels between the different regions are relatively high, and there is some global mobility/travel. Local investments in social capital increase and both social relations and social institutions improve. Learning through local adaptive management has high priority. Technological developments take place at the local level, but knowledge about successful local experiments is easily shared among and between regions. Cultural interaction only highlights the importance of cultural and social diversity. Unfortunately, the fragmentation results in inattention to inequalities. There is no such thing as a global market anymore; trade takes place at the local/regional level. Lower trade levels result in less economic growth. Nevertheless, poverty reduction through education proves to be rather successful, although some regions are lacking behind. Ecosystem functions are highly valued and ecosystems are seen as part of local culture and identity. Governance focuses on national and local management (e.g., certification ecosystem production). There is a diverse mosaic regarding ecosystem

\footnotetext{
${ }^{2}$ The current developed world becomes divided into two groups. The Sustainers are those countries that remained high levels of social and human capital, while the Northern Sleepers had a high potential for local proactive ecosystem management at the turn of century, but this capability is lost over time. The current developed world can also be divided into two groups. The Pumas started with low capacity for proactive, local ecosystem management, but this capacity grows significantly during the scenario. The Trapped do not manage to raise their social and human capital.
} 
functioning-both restoration and degradation- and some local breakdowns and failures occur. The limited capacity to address global issues results in largescale environmental problems (e.g., climate change, fisheries, pollution). Ecological feedbacks are not recognised. After 2030, the importance of crossscale learning/management is increasingly acknowledged and eventually international cooperation increases (e.g., local and global knowledge is combined to address Sub-Saharan malaria) and global trade barriers start to erode. The global action to combat global change is slow in progress though.

These envisioned developments in the contextual and distal level of health determinants affect the multiple proximal determinants (Table 5.5). There is heterogeneity in health service provision, and the use of traditional health systems increases. Medical innovations and vaccines are not available to all. The improving social conditions result in decreasing rates of violence, alcoholism and intravenous drug use. The demand for environmental friendly and healthy local products increases, resulting in a decline in the local use of fertilisers (an example of a positive effect on the physical environment). The distribution of food supplies becomes more equal. However, pressures (e.g., population growth) on food and water systems and on sanitation increase. The absolute number of calorie-malnourished children grows until 2020. Water stress increases in many areas, especially in the developing world. New diseases outbreaks and antibiotic-resistance pose a high risk. Additionally, the risk of major climate change impacts is high in this future.

Table 5.5: Adapting mosaic- developments in proximal health determinants.

\begin{tabular}{|c|c|c|}
\hline $\begin{array}{l}\text { Proximal health } \\
\text { determinants }\end{array}$ & Developed regions & Developing regions \\
\hline Health services & Mixed results & $\begin{array}{l}\text { Mixed results } \\
\text { Medical innovations not available } \\
\text { to all }\end{array}$ \\
\hline $\begin{array}{l}\text { Social environment } \\
\text { \& support }\end{array}$ & Improving & Improving, but some lack behind \\
\hline Lifestyles & Healthy lifestyles possibly increase & Healthy lifestyles possibly increase \\
\hline Violence & Decreasing & Decreasing \\
\hline Water & Increasing water stress & $\begin{array}{l}\text { Significantly increasing water } \\
\text { stress }\end{array}$ \\
\hline Food & Adequate availability & $\begin{array}{l}\text { Declining per capita, but more } \\
\text { equal distribution } \\
\text { Malnutrition increases until } 2020\end{array}$ \\
\hline $\begin{array}{l}\text { Physical } \\
\text { environment }\end{array}$ & $\begin{array}{l}\text { Local improvements, but negative } \\
\text { effects of global changes } \\
\text { New drug-resistant diseases }\end{array}$ & $\begin{array}{l}\text { Local improvements, but negative } \\
\text { effects of global changes } \\
\text { New drug-resistant diseases }\end{array}$ \\
\hline
\end{tabular}


A future without health? | 141

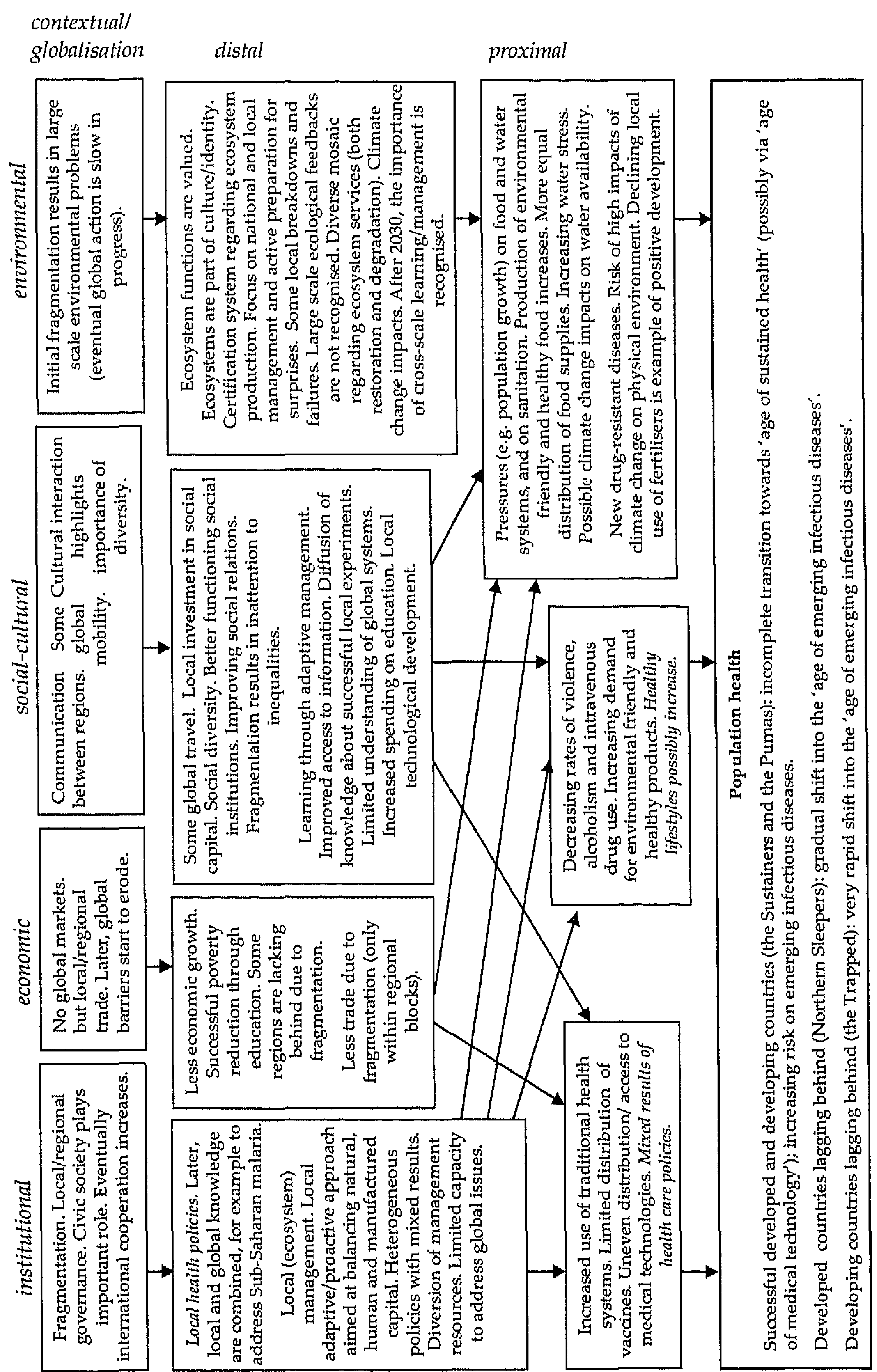

Figure 5.4: Future health in Adapting Mosaic.

Note: Developments that were not specifically mentioned in the MA-documents, but evolved from our interpretation of the combination of the described developments in other relevant factors, are indicated by italics. 
Based on Figure 5.4 and Table 5.5 we conclude that the most successful countries (The Sustainers and the Pumas) will experience great improvements in both social/human capital and the local environment. They move towards the 'age of sustained health', but this transition remains incomplete due to the neglect of global (environmental) issues and the existing inequalities. Some of the current developed countries are less successful (the Northern Sleepers) and their declining capacity for local (ecosystem) management results in a gradual shift into the 'age of emerging infectious diseases'. The developing countries that are left behind (The Trapped) will probably shift into to the age of emerging infectious diseases' rapidly due to, for example, lacking health services, ecosystem failures, and increasing severe water stress. It is important to note that this future also brings a significant risk to be considered. Although many countries will achieve health improvements, the neglected global developments (e.g., environmental change, inequity) will eventually increase the risk of disease outbreaks in the more successful regions as well (due to the significant risk of major ecological breakdowns and surprises), shifting all regions into the 'age of infectious diseases'.

\section{Future health in TechnoGarden}

In the TechnoGarden scenario, globalisation is characterised by a reliance on technological solutions and a focus on environmental concerns. Global markets increasingly expand. Global governance structures and organisations (e.g., professional organisations, transnational corporations) develop a strong set of global (environmental) treaties and large-scale health networks. Globalisation results in global communication and mobility. Travel and migration is easy. An interconnected diverse society emerges (but local cultures are at risk). However, social exclusion also increases, as social issues have less priority. Policies are proactive and based on market mechanism. Global trade is combined with strict monitoring. Economies grow, but a lower rate compared to the Global Orchestration scenario. Poverty declines, but the rich-poor gap remains huge as some regions lack behind. The diffusion of information, knowledge and technology is high. There is a focus on ecosystem research and (health) education. The development of green technologies (e.g., ecosystem engineering, alternative energy, GMOs) has high priority in both developed and developing regions. The global environment improves (e.g., high seas, atmosphere, rivers, wildlife) and part of the climate change is offset. Provision of ecosystem goods and services is high, but often pushed to limits due to reliance on technology. Ecosystems are highly engineered resulting in biodiversity loss and increasing vulnerability. Unexpected secondary effects of technology pose new problems. In this future, some regions are excluded from the global society. Tensions result from existing inequities, and conflicts arise in the isolated regions resulting in ecosystem decline. 
These envisioned developments in the contextual and distal level of health determinants affect the multiple proximal determinants (Table 5.6). Technological innovations result in healthy technologies that are available to most. Due to the proactive approach characterising this future, prevention of diseases will probably have a high priority. However, calorie dense diets and sedentary lifestyles could increase obesity. There is a general decline in social and family relations and a possible associated decline in social support. The regions that are lagging behind have an increased risk of violence. Eco-friendly lifestyles are stimulated, but the main focus lies on technological solutions. Water and air quality, urban sanitation and nutrition greatly improve. Water stress decreases in the OECD and the former Soviet Union, but it increases in many other parts of the world (although increasing more slowly than in the other scenarios). There is an increasing demand on ecological/multifunctional agriculture, which could benefit the physical environment. The liveability of cities improves. Technology-driven changes in ecosystems, however, increase the risk of disease emergence. Additionally, some regions are left behind (e.g., parts of Sub-Saharan Africa, Central Asia); they face significant problems with water quality, droughts, and malnutrition.

Table 5.6: TechnoGarden- developments in proximal health determinants.

\begin{tabular}{|c|c|c|}
\hline $\begin{array}{l}\text { Proximul hedr } \\
\text { deterninants }\end{array}$ & Developed regions & Developing regions . \\
\hline Health services & $\begin{array}{l}\text { Improvements in quality and } \\
\text { access }\end{array}$ & $\begin{array}{l}\text { Strong improvements in quality } \\
\text { and access } \\
\text { Some regions significantly lag } \\
\text { behind }\end{array}$ \\
\hline $\begin{array}{l}\text { Social environment } \\
\& \text { support }\end{array}$ & General decline & General decline \\
\hline Lifestyles & Increasingly unhealthy & Increasingly unhealthy \\
\hline Violence & Declining rates & $\begin{array}{l}\text { Declines rates } \\
\text { Some regions experience conflicts }\end{array}$ \\
\hline Water & $\begin{array}{l}\text { Water withdrawals/stress declines } \\
\text { Increasing quality and adequate } \\
\text { sanitation }\end{array}$ & $\begin{array}{l}\text { Improved quality/sanitation } \\
\text { Some regions face increasing water } \\
\text { problems }\end{array}$ \\
\hline Food & Adequate availability & $\begin{array}{l}\text { Strongly improving nutrition } \\
\text { Some regions face malnutrition }\end{array}$ \\
\hline $\begin{array}{l}\text { Physical } \\
\text { environment }\end{array}$ & $\begin{array}{l}\text { Improving quality } \\
\text { Increased liveability of cities } \\
\text { Risk of emerging infectious } \\
\text { diseases }\end{array}$ & $\begin{array}{l}\text { Improving quality } \\
\text { Increased liveability of cities } \\
\text { Risk of emerging infectious } \\
\text { diseases } \\
\text { Some regions face deteriorating } \\
\text { living environments and high risk } \\
\text { of disease emergence }\end{array}$ \\
\hline
\end{tabular}


144

Chapter 5

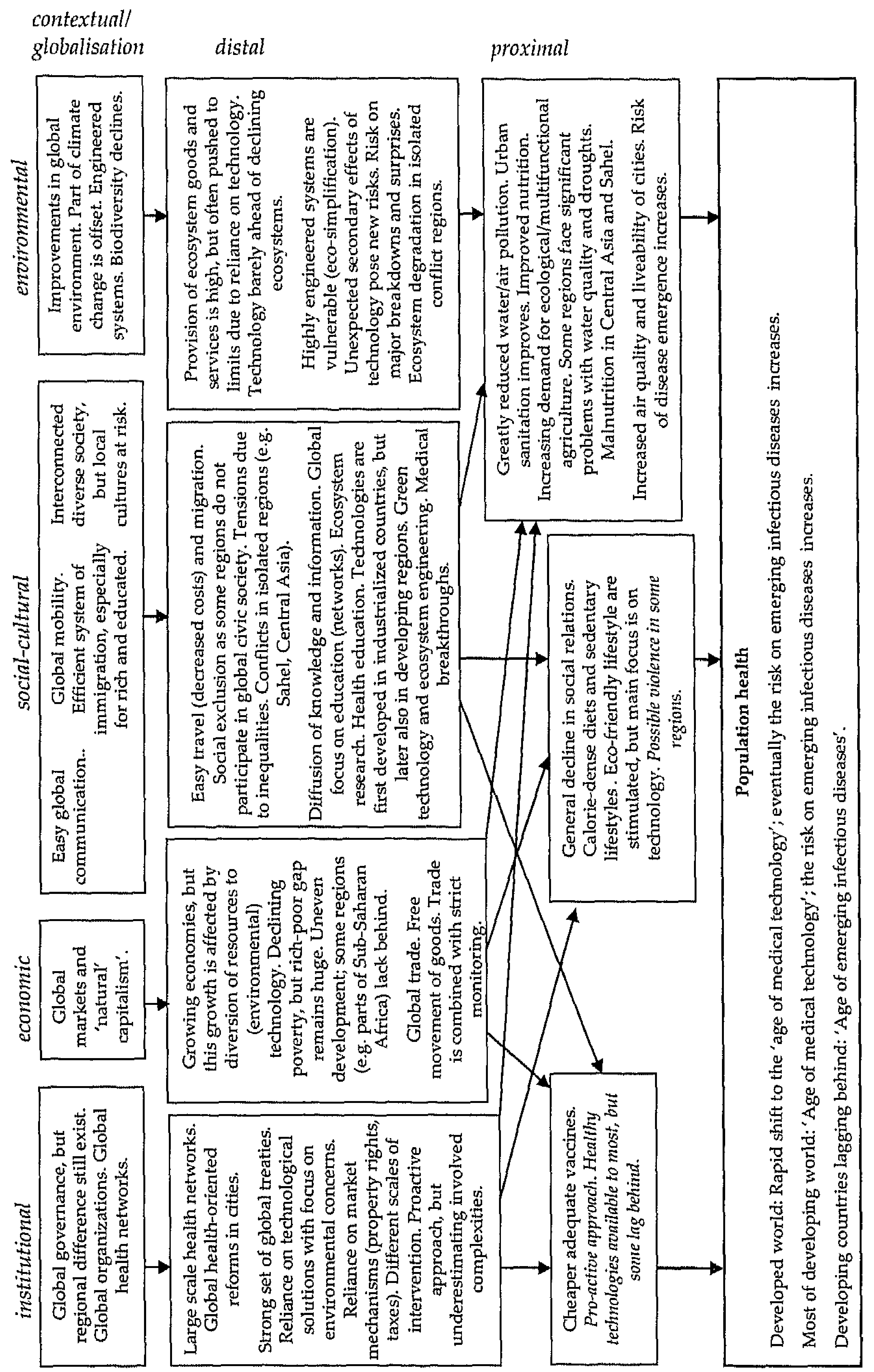

Figure 5.5: Future health in TechnoGarden.

Note: Developments that were not specifically mentioned in the MA-documents, but evolved from our interpretation of the combination of the described developments in other relevant factors, are indicated by italics. 
Based on Figure 5.5 and Table 5.6 we conclude that the developed world will advance into the 'age of medical technology' rapidly. Despite the lower economic growth, technological advances, among other things, are expected to result in some significant health improvements. Large parts of the current developing world will also experience some great health improvements and technological developments, advancing to the 'age of medical technology'. Both regions also face, however, unhealthy lifestyles and a coexisting risk of emerging infectious diseases. Additionally, the uneven development in this scenario causes other parts of the developing world (e.g., Sahel, Central Asia) to face a negative spiral of violence, ecosystem degradation, emerging diseases and poverty, resulting into the 'age of emerging infectious diseases'. It is important to note that this future also brings a significant risk to be considered. The complexities involved are underestimated and technology is barely ahead of the declining ecosystems. Additionally, secondary effects of technologies pose a significant risk to the highly vulnerable engineered ecosystems. So there is a significant risk of major ecological breakdowns and surprises in this future, and a consequent risk of shifting all regions into the 'age of emerging infectious diseases'.

\subsubsection{Future health in a globalising world}

So how will the globalisation process and population health evolve in the future? The futures of the selected scenarios are diverse, and we must keep in mind that the timeframes of these scenario groups differ. However, Table 5.7 shows that beneath the diversity in scenario names and the narrative motivation for each storyline lays a common set of archetypal globalisation pathways: a globalising world with a market-oriented economic focus, a globalising world with a 'sustainability' focus, and a fragmented world (i.e., retreat of globalisation). Each group has two main variants.

Apparently, there is some kind of 'consensus' about the variety of possible globalisation futures. This section focuses on the SRES-, GEO3- and MAscenarios. Based on the previous sections, we anticipate the following future developments in health.

- The first group of scenarios describes a globalising world with an economic focus. In the future of Markets First (GEO3), the global market-based approach is only able to partly mitigate social and environmental problems; the developing countries are likely to slowly move towards the 'age of emerging infectious diseases', while the developed world manages to advance to the 'age of medical technology'. A1 (SRES), on the other hand, is more optimistic about the mitigation capacity of global economic and technological developments; developing countries will experience 
improvements in health and increased life expectancy, but at the same time they will face the risk of emerging infectious diseases. Global Orchestration (MA) is also more optimistic about the mitigation of social problems in particular. It is important to note, however, that in this scenario the rich are eventually facing increasing environmental problems and a subsequent risk of emerging infectious diseases.

Table 5.7: The future of globalisation: globalisation pathways in selected global scenario studies.

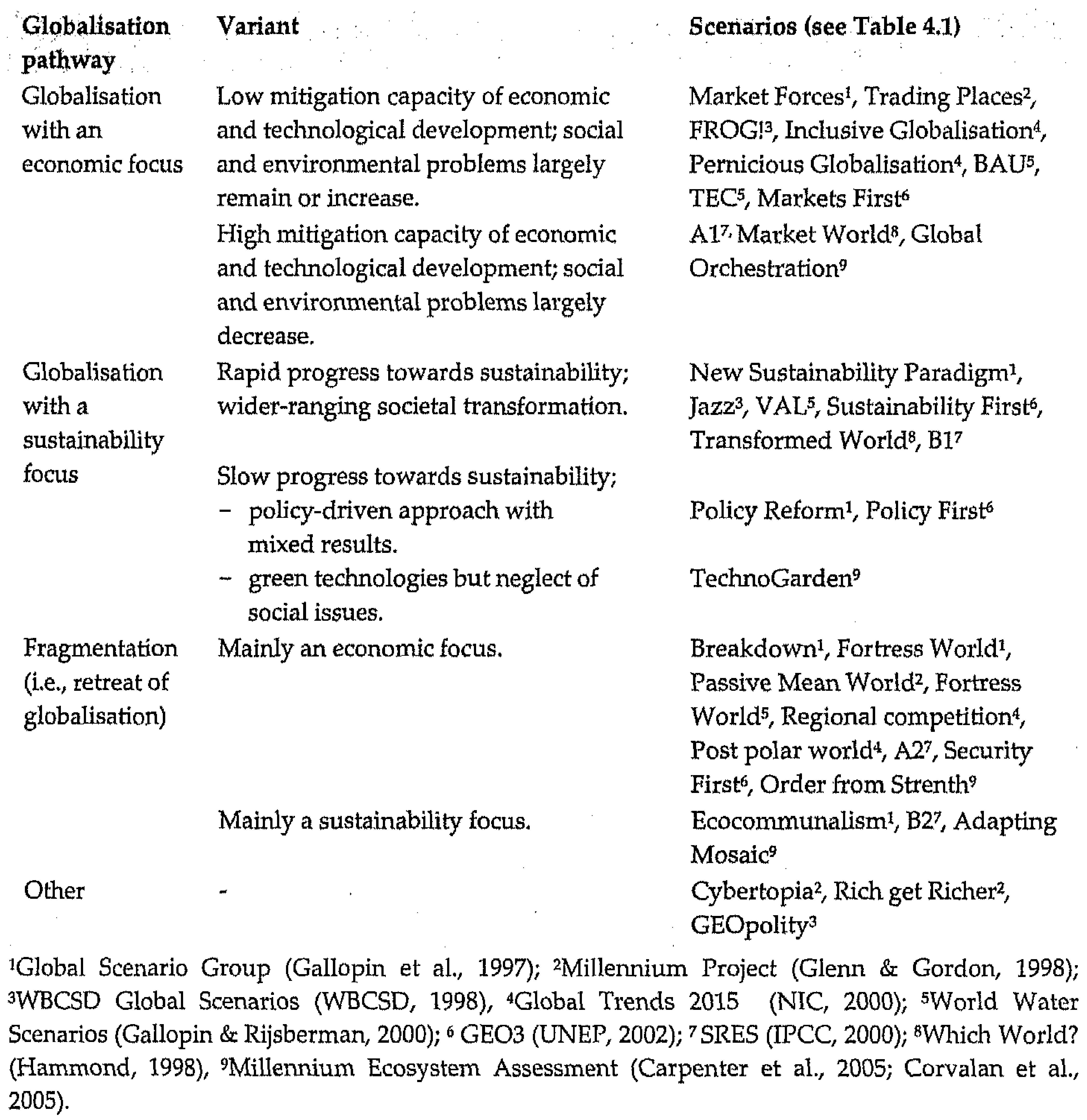

Low mitigation capacity of economic and technological development; social and environmental problems largely remain or increase.

High mitigation capacity of economic and technological development; social and environmental problems largely decrease.

Rapid progress towards sustainability; wider-ranging societal transformation.

Slow progress towards sustainability;

- policy-driven approach with mixed results.

- green technologies but neglect of social issues.

Mainly an economic focus.

Mainly a sustainability focus.

\section{Scenarios (see Table 4.1)}

Market Forces 1 , Trading Places2, FROG! ${ }^{3}$, Inclusive Globalisation ${ }^{4}$ Pernicious Globalisation", BAU5, TEC ${ }^{5}$, Markets First ${ }^{6}$

A17, Market World 8 , Global Orchestration ${ }^{9}$

New Sustainability Paradigm1, $\mathrm{Jazz}^{3}, \mathrm{VAL}^{5}$, Sustainability First ${ }^{6}$, Transformed World ${ }^{8}, \mathrm{B1}^{7}$

Policy Reform ${ }^{1}$, Policy First ${ }^{6}$

TechnoGarden 9

Breakdown ${ }^{1}$, Fortress World1, Passive Mean World ${ }^{2}$, Fortress World5, Regional competition, Post polar world ${ }^{4}, \mathrm{A2}^{7}$, Security First', Order from Strenth ${ }^{9}$ Ecocommunalism ${ }^{1}, \mathrm{B2}^{7}$, Adapting Mosaic ${ }^{9}$ Cybertopia2, Rich get Richer2, GEOpolity ${ }^{3}$

Other

${ }^{1}$ Global Scenario Group (Gallopin et al., 1997); ${ }^{2}$ Millennium Project (Glenn \& Gordon, 1998); ${ }^{3}$ WBCSD Global Scenarios (WBCSD, 1998), ${ }^{4}$ Global Trends 2015 (NIC, 2000); 5 World Water Scenarios (Gallopin \& Rijsberman, 2000); ${ }^{6}$ GEO3 (UNEP, 2002); 7 SRES (IPCC, 2000); ${ }^{8}$ Which World? (Hammond, 1998), 9Millennium Ecosystem Assessment (Carpenter et al., 2005; Corvalan et al., 2005). 
- In the scenarios describing a globalising world with a focus on (environmental) sustainability, we also distinguish two variants. In B1 (SRES) and Sustainability First (GEO3), society is very successful in balancing environmental, social, and economic goals; both developing and developed countries are likely to advance to the 'age of sustained health'. However, in the future described in Policy First (GEO3) and TechnoGarden (MA) the global advances towards sustainability are slow, but each for different reasons. Policy First is characterised by a mere policy-driven approach to sustainability with mixed results. The developing countries are not likely to advance beyond the 'age of medical technology' or even the 'age of chronic diseases'. Sooner or later, they will face a growing risk of disease outbreaks. Developed countries slowly progress via the 'age of medical technology' towards the 'age of sustained health', but are not able to complete the transition to a sustainable society. In TechnoGarden, the whole society acknowledges the importance of healthy ecosystems, but neglect important drawbacks of technology and important social issues such as equity. The developed world will rapidly progress to the 'age of medical technology', but will not advance to the 'age of sustained health'. Some developing countries also experience great technological improvements as well, but others are left behind ('age of emerging infectious diseases').

- The scenarios unfolding in a fragmented world with an economic approach- A2 (SRES), Security First (GEO3) and Order from Strength (MA)can be related to a future where the developed world is likely to advance to the 'age of medical technology', but also experience an increased risk of infectious disease. The developing countries shift into the 'age of emerging infectious diseases'. Alternatively, in the fragmented futures of B2 (SRES) and Adapting Mosaic (MA) there is some local and slow progress in achieving sustainability and the 'age of sustainable health' in the developed world, but the transition is not complete. In developing countries, health improves, but the pace is too slow for a shift beyond the 'age of chronic diseases'. Some developing countries might achieve some modest technological progress by themselves. The threat of emerging infectious diseases becomes a reality for many of the less successful countries.

\subsection{DISCUSSION}

The inclusion of health in past global scenario exercises has been limited. However, looking at other developments that possibly affect our future health (e.g., food, water, environment, social change, equity, economic growth, technology), we see that many are well addressed in most scenarios. It, 
therefore, would have been possible to describe future developments in health as an outcome of these multiple drivers and pressures.

Given that health is regarded by many as one of the most important assets of the human life and an important component of human security, why has there been so little effort in the past decade of scenario development to explicitly address human health? From the point of view of the global scenario community, exploring the potential health impacts of global changes poses a difficult challenge; as health is an integrating bottom-line outcome, scenario builders might hesitate to include such a complex and multi-causal issue into their studies. From a public health point of view, exploration of these global, long-term and complex risks to human health seems far removed from the tidy examples that abound in textbooks of epidemiology and public health research. It is, therefore, difficult to engage epidemiologists and other population health scientists in this unfamiliar domain. As a result, health is only beginning to play a role in global scenario assessments.

Two main approaches to develop health scenarios can be distinguished (Huynen, 2003). First, one could develop new integrated health scenarios from scratch. This would be, of course, very challenging, and it would be possible to make use of the expertise already available in the scenario community. The second approach builds on the outcomes of earlier studies and constitutes enriching existing global scenarios with a health component.

This Chapter describes a first-order attempt to add a health dimension to existing global scenarios in order to explore the health effects of future globalisation. It provides useful insights into how to deal with health in scenarios and shows that a comprehensive picture of future health evolves when all relevant social-cultural, institutional, economic, and environmental developments are taken into account. However, in order to connect current scenarios to a more robust analysis of changes in health outcomes, supplementary analysis is required. An additional step, for example, would be enriching the narrative storylines through a participatory process with stakeholders and/or the (semi-) quantification of narrative storylines by means of modelled scenarios.

The MA (Carpenter et al., 2005) concludes that in the near future, enriching (global) scenarios - often in collaboration with key stakeholders- will define an important agenda for policy analysis, scientific research and education. This is certainly the case for the very relevant field of 'global health': narratives need to include richer descriptions of the diverse linkages between globalisation and health, and, ideally, models will be developed to quantitatively simulate these interactions. Although we believe that the current study provides an important step forward in exploring the future health impacts of globalisation, further research is needed to improve our (quantitative) understanding of the connections among global processes, the multiple distal and proximal health 
determinants, and our health. We anticipate that this study provides a platform for beginning to address these challenges. 


\section{Chapter 6}

\section{CLIMATE CHANGE, TEMPERATURE AND MORTALITY IN THE NETHERLANDS}

Section 6.2 is based on:

- Huynen, M.M.T.E., Martens, P., Schram, D., Weijenberg, M.P. \& Kunst, A.E. (2001). The impact of heat waves and cold spells on mortality rates in the Dutch population. Environmental Health Perspectives, 109(5), 463-470. 


\section{CLIMATE CHANGE, TEMPERATURE AND MORTALITY IN THE NETHERLANDS}

Globalisation can affect population health through the impacts of global environmental change (Martens et al., 2000). The increasing energyintensiveness and scale of human economic activities over the past two centuries have altered our global atmosphere, due to interference with the climate regulation and carbon storage functions of the planet's ecosystems. The Intergovernmental Panel on Climate Change (IPCC) (2007b) states that global greenhouse gas (GHG) emissions (e.g., carbon dioxide, methane and nitrous oxide) have grown significantly since pre-industrial times, with a very strong increase since 1970. Although some uncertainties remain about the sensitivity of the climate system to changes in GHG concentrations, and about the future trajectories of these concentrations, there is now near-unanimous scientific agreement that the rising GHG concentrations will cause additional warming and other climatic changes (including a change in climatic variability) over coming decades (Canfalonieri \& McMichael, 2006; IPCC, 2007b). According to the recent IPCC fourth assessment report (IPCC, 2007b), the expected increase in global mean temperature is between 1.1 and $6.4^{\circ} \mathrm{C}$ for the year 2100 .

As a result, the past decade have witnessed an increasing concern regarding the health impacts of anthropogenic climate change (see, for example, Patz et al. (2006) and McMichael et al. (2006)). Climate plays an important role in environmental variability and change, and, consequently, plays an important role in the epidemiology of diseases (Comrie, 2007). The link between weather and human health is, for example, illustrated by the fact the El Niño cycle is associated with increasing rates of some diseases, such as malaria and cholera (Kovats et al., 2003; Pascual et al., 2000). Global climate change is likely to influence human health in various ways (Figure 6.1). Although some effects may be beneficial, most are expected to be adverse. Some impacts will occur via direct mechanisms (e.g., temperature-related morbidity and mortality); others will occur through indirect mechanisms (e.g., transmission of vector-borne diseases).

This Chapter focuses on the direct effects of temperature (change) on mortality in the Netherlandsi. We assess the impact of outdoor temperature on mortality in the period 1979-1997, including the impact of heat waves and cold spells (section 6.2). Accordingly, we explore the effects of climate change on the temperature-related mortality, using recently developed climate change scenarios for the Netherlands (section 6.3).

\footnotetext{
1 For an overview of other health effects of climate change in the Netherlands see, for example, Huynen (2006), and Bresser et al. (2005).
} 


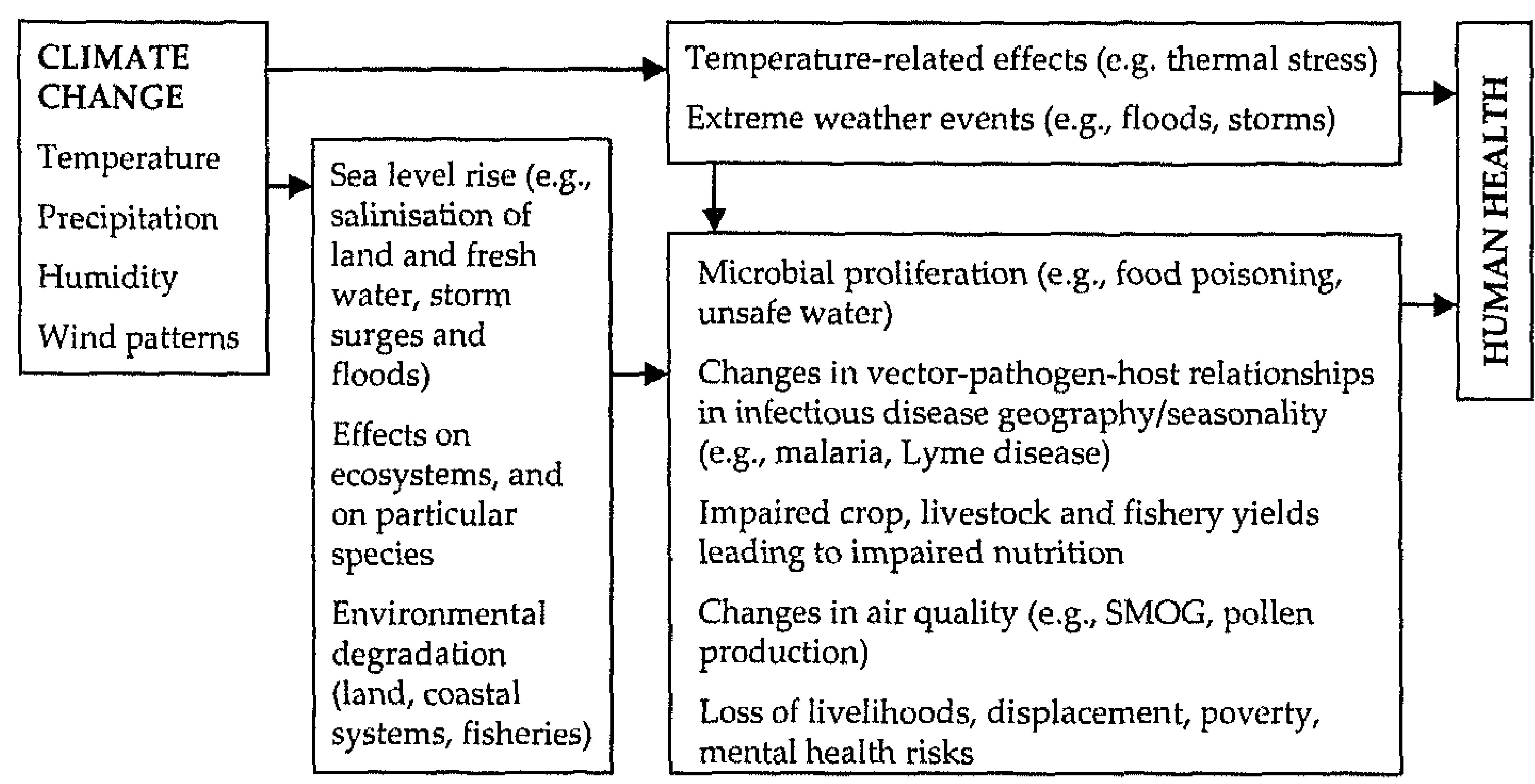

Figure 6.1: Climate change is likely to influence human health in various ways (adapted from McMichael et al. (2006)).

\subsection{Climate CHANGE, TEMPERATURE AND MORTALITY}

Many studies (Alberdi et al., 1998; Ballester et al., 1997; Kunst et al., 1993; Pan et al., 1995; Rooney et al., 1998) have shown the relationship between temperature and mortality; this relationship has a 'V shape', or 'U-shape', with mortality rates lower on days with a temperature closer to the temperature level corresponding to the lowest point of the curve. The prevailing climate of a geographical area determines the level of this optimum temperature level. The projected change in climate will influence both annual cold-related and heatrelated mortality. As expected, cold winters are estimated to become rarer with global warming, and hot summers become much more frequent.

Besides the increase in average temperatures, climate change is also expected to affect the occurrence, intensity and duration of heat waves and cold spells (IPCC, 2007b). Since 1950, the number of heat waves has increased. In a warmer future climate, there will be an increased risk of more intense, more frequent and longer-lasting heat waves. The European heat wave of 2003 is an example of the type of extreme heat event lasting from several days to over a week that is likely to become more common in a warmer future climate (IPCC, 2007b). Over the 20th century, human influences more than doubled the risk of having a summer in Europe as hot as that of 2003; in the absence of human influences, the risk would probably have been one in many hundred years (IPCC, 2007b; Stott et al., 2004). 
Table 6.1: Increased mortality during heat waves: some examples.

Heat wave
London, July-August 1995 (Rooney et al.,
1998)
Belgium, 1994 (Sartor et al., 1995)

Czech Republic, 17 heat waves during 19822000 (Kysely, 2004)

Switzerland, 2003 heat wave (Grize et al., 2005)

France (13 cities), 2003 heat wave (Pirard et al., 2005)

Italy ( 4 cities) 2003 heat wave (Michelozzi et al., 2005)
Impact on mortality

$15 \%$ increase in mortality

$13.2 \%$ increase in mortality in the elderly; $9.2 \%$ increase among those aged $0 / 64$ years

$13 \%$ and $14 \%$ increase for total mortality respectively cardiovascular mortality

$7 \%$ increase in mortality

$60 \%$ increase in mortality; from $142 \%$ in Paris to $4 \%$ in Lille

Increase in all cities, highest for Milan (23\%) and Turin $(33 \%)$

An increase in the number of deaths has been recorded during summer heat waves (see Table 6.1 for examples). Heat places stress on the thermoregulatory system (Alberdi et al., 1998). Exposure to high temperatures causes increases in blood viscosity and blood cholesterol levels (Kunst et al., 1993). Much of the excess mortality attributable to heat waves is attributable to increases in deaths from cardiovascular, cerebrovascular and respiratory disease. The elderly are most affected.

Still, the figures need to be put into perspective, as not all heat-related deaths results in significant loss of life; part of the excess mortality during heat waves must be viewed as 'only a slight forward displacement of deaths'. As a result of this forward displacement of deaths, a temporary fall in mortality is often observed in the weeks following a heat wave (e.g., Sartor (1995)). This phenomenon is also called 'early harvesting' when a proportion of deaths that occur during a heat wave occur in susceptible persons - the frail or sick - who were likely to have died in the near future. In other words, it is often 'the last straw' of additional stress for those persons already suffering from compromised health (Comrie, 2007). The other part of the excess mortality, however, relates to avoidable deaths and substantial loss of life. It has been estimated that this forward displacement of deaths possibly account for $20-50 \%$ of the excess mortality observed during heat waves (Ebi et al., 2004). However, no clear drop in mortality has been observed in the months following the severe 2003 heat wave in France (Pirard et al., 2005).

Furthermore, many questions about the interaction between high temperature and high concentration of air pollutants still need to be resolved. A study by Fischer et al. (2004), for example, concluded that in the Netherlands about $40 \%$ of the deaths now being attributed to the hot summer weather in 2003 (June- 
August) can reasonably be expected to have been caused by air pollution during this period.

Cold temperatures have been related to increases in mortality during winter in a number of studies in Europe (Carder et al., 2005; Diaz et al., 2005; Eurowinter Group, 1997; Keatinge \& Donaldson, 2001; Kunst et al., 1993; Rossi et al., 1999; Wilkinson et al., 2004), as well as in the United States (Keatinge \& Donaldson, 2001), and in other countries (El - Zein et al., 2004; O'Neill et al., 2005). Exposure to cold can lead to direct cardiovascular stress due to changes in blood pressure, vasoconstriction, and an increase in blood viscosity and levels of red blood cell count, plasma cholesterol, and plasma fibrinogen (Alberdi et al., 1998; Keatinge \& Donaldson, 1995). Low temperatures lead to thrombosis due to hemoconcentration (Donaldson \& Keatinge, 1997; Eurowinter Group, 1997), and rapid deaths occur due to the rupture of atheromatous plaques during hypertension and cold-induced coronary spasm (Eurowinter Group, 1997). Indirectly, influenza might contribute to cold-related mortality (Alberdi et al., 1998; Anderson \& Le Richie, 1970; Kunst et al., 1993). Susceptibility to pulmonary infections may increase through bronchoconstriction, caused by breathing cold air (Schaanning et al., 1986). However, the precise role of cold temperatures in the seasonality of influenza is yet to be determined (Comrie, 2007). In the future, many countries with a high proportion of deaths in winter are likely to experience a reduction in excess winter mortality from milder winters under climate change. Langford and Bentham (1995) estimated that 9,000 wintertime deaths per year could be avoided by the year 2025 in England and Wales under a $2.5^{\circ} \mathrm{C}$ increase in average winter temperature. A metaanalysis by Martens (1998b) estimated that an increase in global temperature could result in a reduction in winter cardiovascular mortality in Europe.

\subsection{TEMPERATURE AND MORTALITY IN THE NETHERLANDS}

In this section, we investigate the impact of outdoor temperature on mortality in the Netherlands during 1979-1997, as well as the impact of cold spells and heat waves on mortality. We also examine whether the cold spells and heat waves merely brought forward the deaths of those who would have died in the short term anyway or if the induced mortality made a substantial contribution to overall lost lifetime. 


\subsubsection{Methods ${ }^{2}$}

\section{Data}

Statistics Netherlands provided the numbers of deaths by the day on which the death occurred (1 January 1979- 31 December 1997) and by selected causes of death and two age categories (0-64 years of age and $\geq 65$ years of age, only for 1 January 1988-31 December 1997). The selected causes of death were malignant neoplasms (International Classification of Diseases, Revision 9, ICD-9: AM 1219), respiratory disease (ICD-9: AM 33-35), and cardiovascular disease (ICD-9: AM 25-32).

The Netherlands Royal Meteorological Institute (KNMI) provided 24-hour data on minimum and maximum temperatures. The average daily temperature was calculated as the average of the minimum and maximum temperatures. All data refer to the De Bilt station, which is located in the centre of the country. Differences in climate within the Netherlands are small, and weather changes usually affect all parts of the country at roughly the same time. Figure 6.2 illustrates the daily average temperatures and total mortality rates in the Netherlands in the study period.

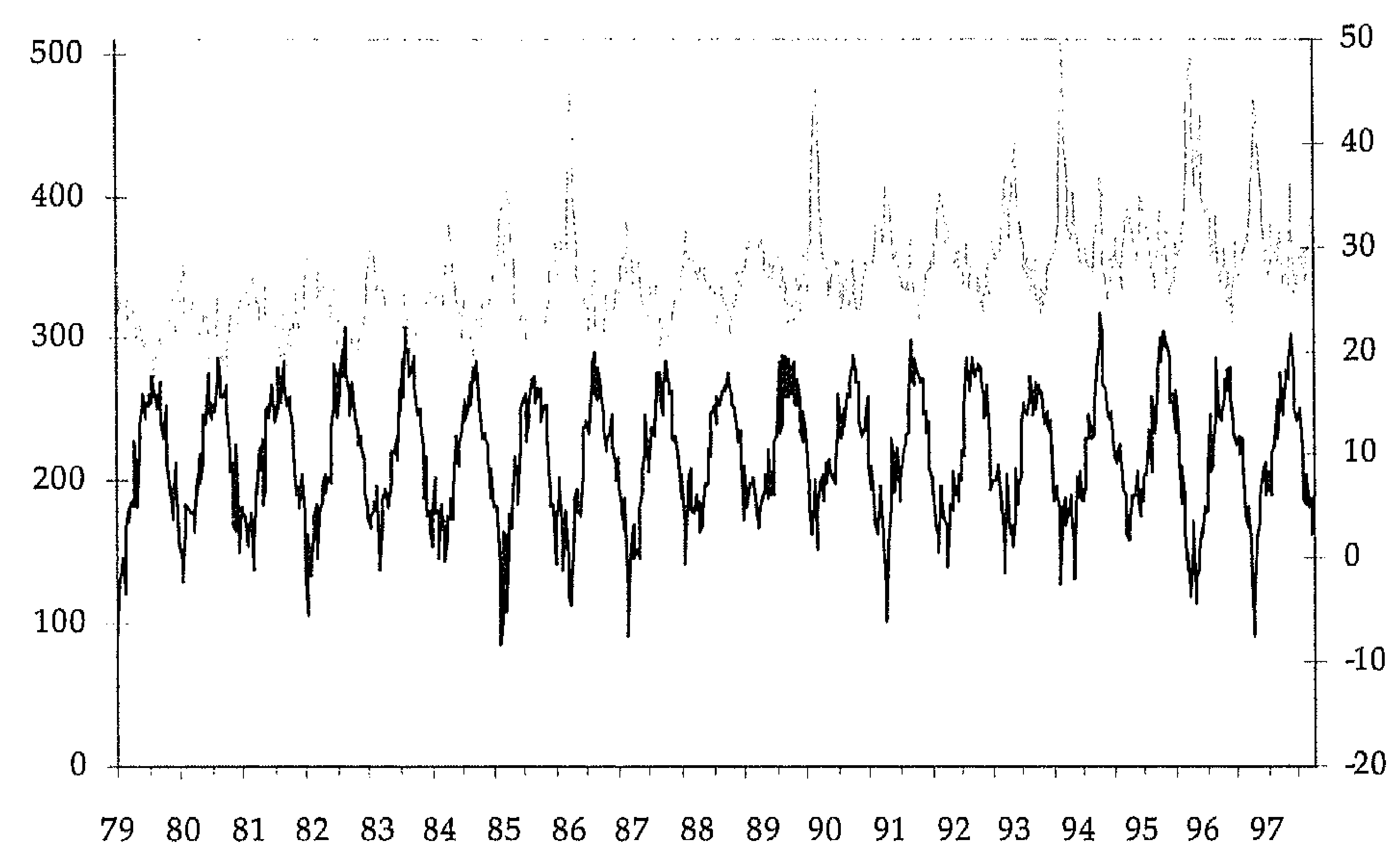

Figure 6.2: Daily average temperatures $\left({ }^{\circ} \mathrm{C}\right)$ and total mortality rates in the Netherlands, 1 January 1979- 31 December 1997.

${ }^{2}$ We used SAS version 6.12 software (SAS Institute, Cary, NC, USA) to analyse the data. 
Definition of heat waves and cold spells

A heat wave is defined by the KNMI as a period of at least 5 days, each of which has a maximum temperature of at least $25^{\circ} \mathrm{C}$, including at least 3 days with a maximum temperature of at least $30^{\circ} \mathrm{C}$ (measured at the De Bilt station). According to this definition, there were six heat waves in the study period, and they lasted from 6 to 13 days (Table 6.2). There is no official definition of a cold spell in the Netherlands. We searched for extreme cold periods between 1 January 1979 and 31 December 1997, which occurred with a frequency that resembled the frequency of the official heat waves in our study period. Because the minimum temperature during the winter months (December-February) correlates more closely with mortality than the maximum temperature, the definition we adopted for a cold spell was based on the daily minimum temperature. In our definition, a cold spell is a period of at least 9 days with a minimum temperature of $-5^{\circ} \mathrm{C}$ or lower, of which at least 6 days have a minimum temperature of $-10^{\circ} \mathrm{C}$ or lower (measured at the De Bilt station) Therefore, the duration of a cold spell according to this definition is longer than that of a heat waves. During the study period, a cold spell occurred five times according to our definition, varying in duration from 9 to 17 days (Table 6.2).

Table 6.2: Heat waves and cold spells in the Netherlands, 1979-1997.

\begin{tabular}{|c|c|c|}
\hline 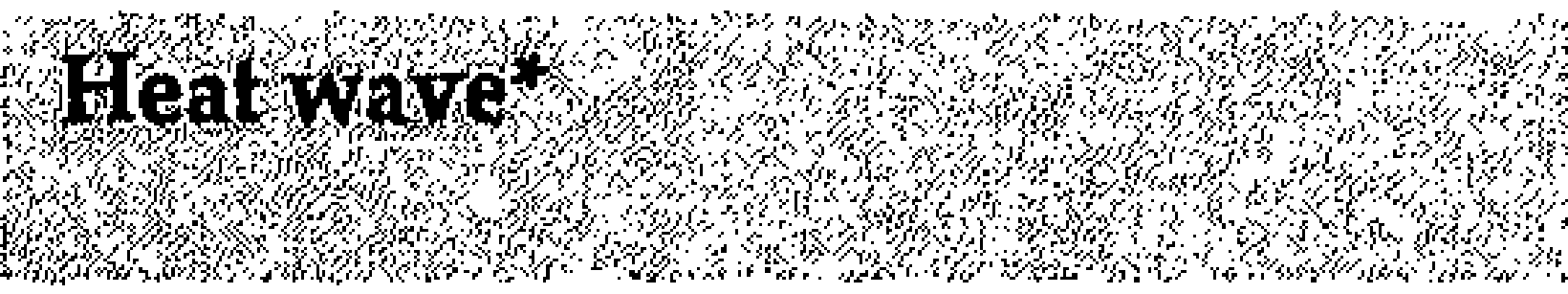 & Moximum tenpenatwe & Maximum temperature \\
\hline 29 July - 4 August 1982 & 3 days & 4 days \\
\hline 4 July - 12 July 1983 & 6 days & 3 days \\
\hline 26 July - 4 August 1990 & 7 days & 3 days \\
\hline 19 July - 31 July 1994 & 8 days & 5 days \\
\hline 29 July - 3 August 1995 & 3 days & 3 days \\
\hline 5 August - 13 August 1997 & 4 days & 5 days \\
\hline 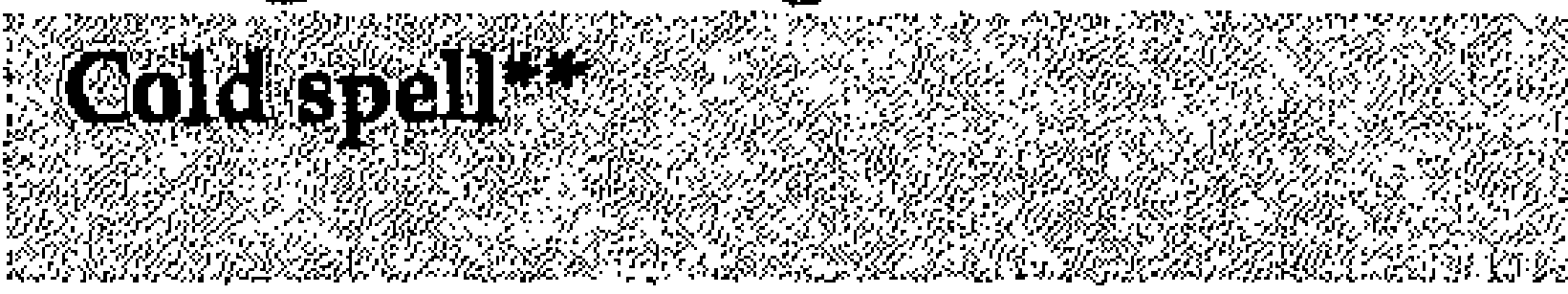 & Minnumim temperature & Minum temperatire at \\
\hline 30 December 1978 - 6 January 1979 & 1 day & 8 days \\
\hline 4 January - 20 January 1985 & 6 days & 11 days \\
\hline 12 February - 28 February 1986 & 11 days & 6 days \\
\hline 6 February - 14 February 1991 & 3 days & 6 days \\
\hline 24 December 1996 - 9 January 1997 & 9 days & 8 days \\
\hline
\end{tabular}

3 The analysis of the excess mortality during cold spells was also performed with another definition of a cold spell, in which the duration of a cold spell was shorter and the requirements regarding the level of the minimum temperature were more stringent. This additional analysis, however, showed no clear excess mortality during these shorter extreme cold periods (results not shown). 
Modelling the association between temperature and mortality

The daily numbers of deaths due to all causes, as well as those from the selected causes, were related to the daily average temperature using Poisson loglinear regression analyses over the whole dataset (1 January 1979-31 December 1997), controlled for the time trend and season. Time trend (the sequential number of the day- 1 for 1 January 1979 and 7,305 for 31 December 1997) was included to account for long-term trends resulting from changes in, for example, population structure, socioeconomic conditions, and the provision of health care over time. Without correcting for season, the mortality effects of seasonal variation in other factors, such as physical exercise, diet, stress, and blood pressure, are picked up by temperature (Kunst et al., 1991; Mackenbach et al., 1992). The variable representing season is kept constant between years (included as a dummy variable for each month, with December as reference month) (Kunst et al., 1993).

Figures 6.3 and 6.4 show the $V$-like relationship between mortality and temperature for mortality due to all causes as well as for mortality due to the selected causes and in the two age groups. Therefore, average daily temperature within the model was measured by two complementary variables, heat ( 0 if average temperature soptimum value, otherwise average temperature minus optimum value) and cold ( 0 if average temperature $\geq$ optimum value, otherwise optimum value minus average temperature). The optimum temperature value corresponds to the average temperature with the lowest mortality level. We performed regression analyses to evaluate several optimum values (e.g., $14.0^{\circ} \mathrm{C}, 14.5^{\circ} \mathrm{C}$, etc.) until we found the one that best fit the lowest scaled deviance. The regression equations for the assessment of the optimum values included values of heat and cold for previous days to account for lagged effects of temperature, in accordance with the study by Kunst et al. (1993). To reduce multicolinearity, temperature variables were constructed for groups of subsequent days (lag periods) by averaging values for heat and cold over these periods. Lag times were grouped into lag periods that increased exponentially in size (1-2, 3-6, 7-14, 15-30 days), whereas lag times longer than 1 month were ignored (Kunst et al., 1993). The regression model can be described by

$$
\log (y)=B_{0}+B_{1}{ }^{*} t_{i}+B_{2}{ }^{*} x_{2}+\ldots+\beta_{j}^{*} x_{j}
$$

where $y$ is the number of deaths on day $i$ (index day), $t_{i}$ is the sequential value for day $i$ (from 1 for 1 January 1979 to 7,305 for 31 December 1997), $x_{2} \ldots x_{j}$ are the $j$ independent variables (values for heat, values for cold, average values for heat during the different lag times, average values for cold during the different lag times, calendar months), and $\beta_{2} \ldots B_{j}$ are the regression coefficients. 


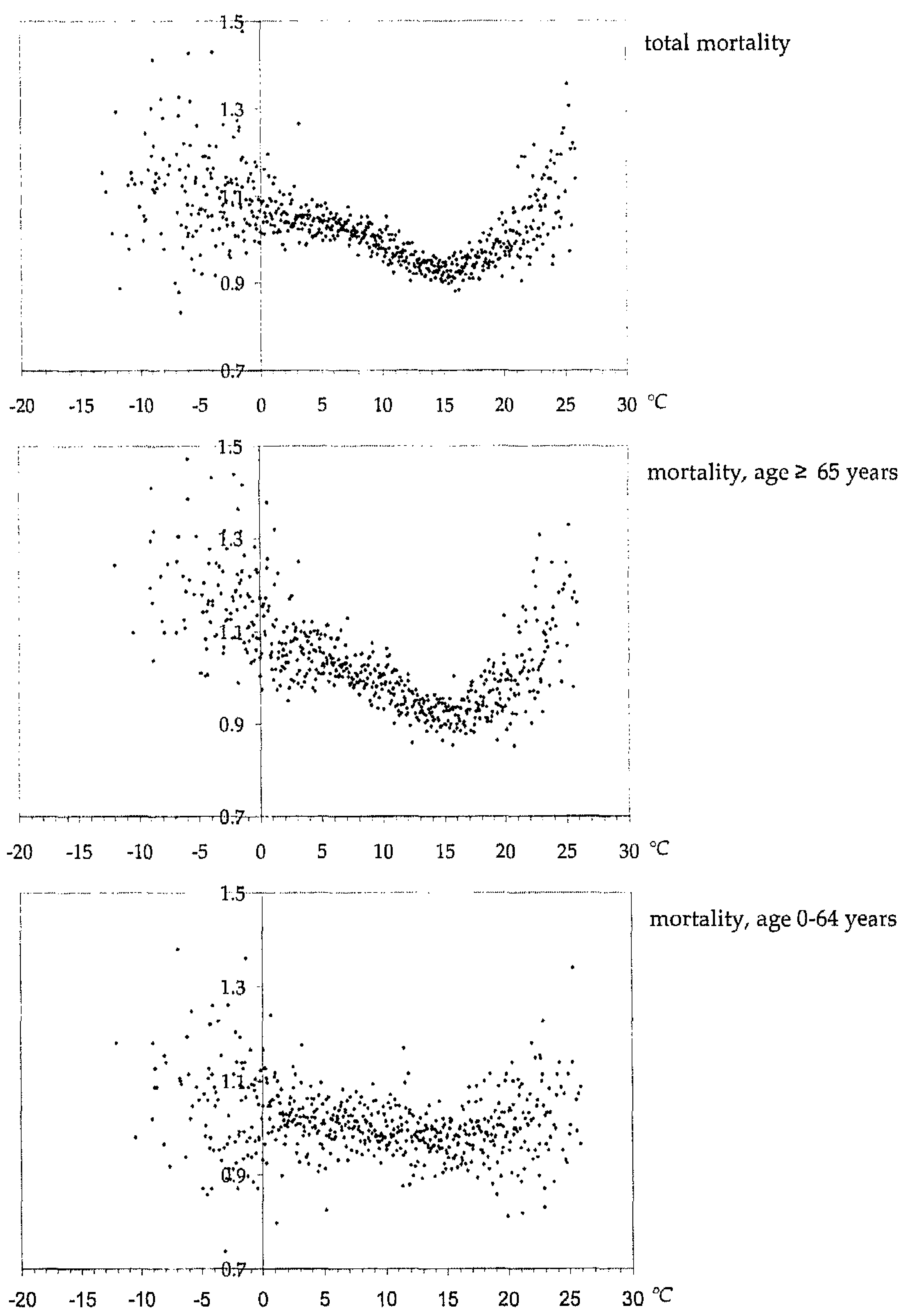

Figure 6.3: The relationship between mortality (total, aged 0-64 years, aged $\geq 65$ years) and average temperature $\left({ }^{\circ} \mathrm{C}\right.$ ), the Netherlands, 1979-1997 (mortality ratio on $Y$-axis $=$ observed number of deaths on day $i /$ mean number of deaths over the whole study period). 
Climate change, temperature and mortality in The Netherlands | 161

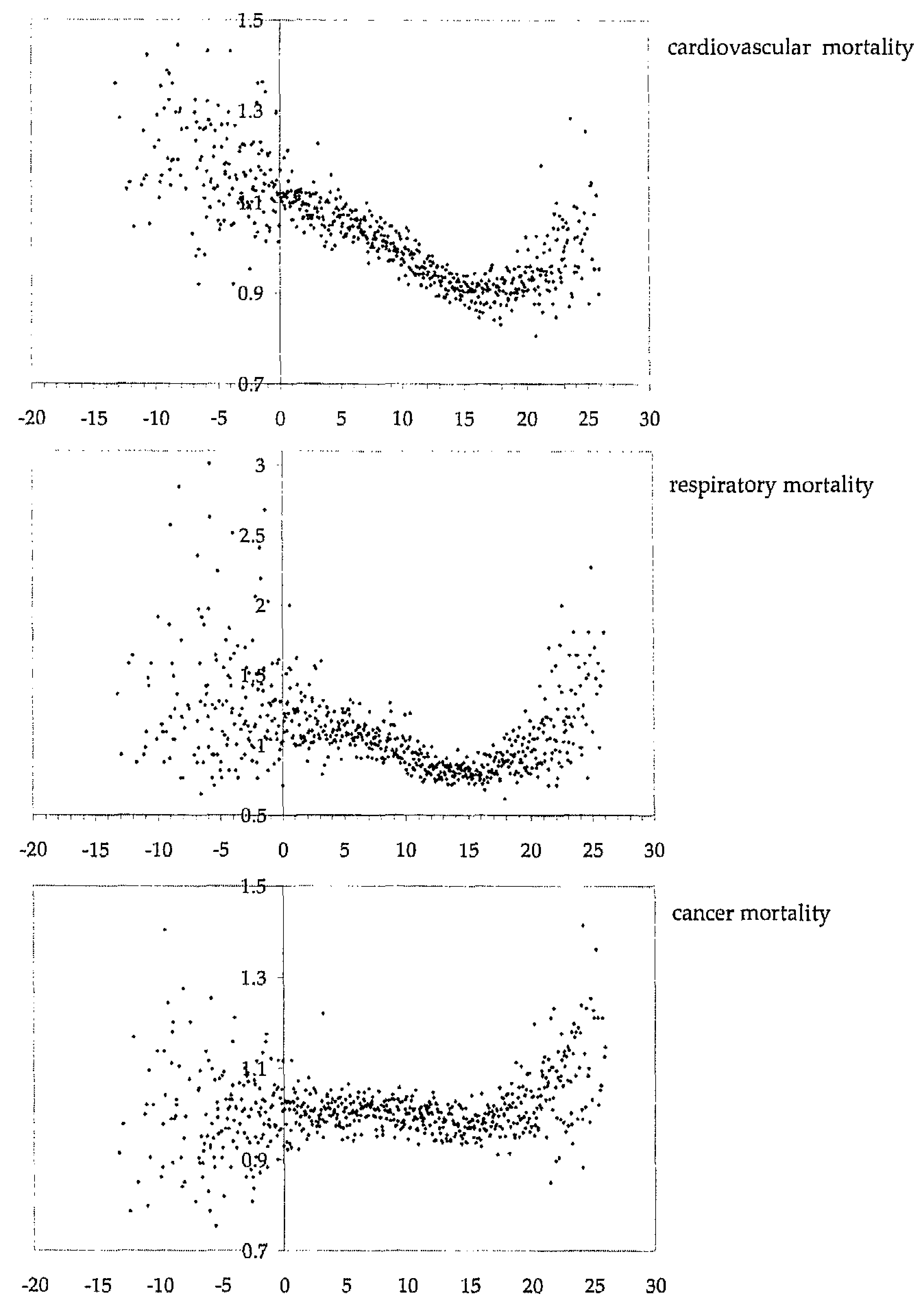

Figure 6.4: The relationship between mortality (cardiovascular, respiratory, cancer) and average temperature $\left({ }^{\circ} \mathrm{C}\right.$ ), the Netherlands, $1979-1997$ (mortality ratio on $Y$-axis $=$ observed number of deaths on day $i$ / mean number of deaths over the whole study period). 
The regression coefficient (B) corresponding to a lag period was transformed, using the formula $100^{*}\left(e^{\beta}-1\right)$, to the percentage change in mortality associated with a $1^{\circ} \mathrm{C}$ increase in the average value of cold or heat within the respective lag period (percental effect). In order to construct an appropriate and parsimonious model for each cause of death and age group, variables were omitted if they met one of the two exclusion criteria: a) adding the variable to the model causes a reduction in the scaled deviance associated with the null model (time trend only) of $<1 \%$, and $b$ ) the regression coefficient corresponding to the variable was not significant $(p=0.01)$.

\section{Excess mortality during cold spells and heat waves}

To examine the impact of extreme temperatures on mortality, we calculated 31day moving averages of daily mortality during the heat wave and cold spell days for the two preceding years combined (Rooney et al., 1998). This was used to estimate the mortality during the heat wave or cold spell period in the absence of extreme temperatures. Excess mortality was calculated as the difference between the total number of deaths observed in the heat wave or cold spell and the corresponding 31-day moving average. Analyses were repeated for the selected age groups and causes of death. Excess mortality could not be calculated for the cold spell in winter 1978-1979, because this cold spell started before our study period began (1 January 1979), and because the necessary mortality data was not available prior to this day.

In addition to the method described above, Rooney et al. (1998) investigated excess mortality during the 1995 heat wave in England using another approach. They compared the observed mortality with the 31-day moving average for the same year. The results for excess mortality during heat waves and cold spells using this method are reported in this paper but not discussed because we believe that this approach is less accurate. It is certainly more conservative, as the values for heat wave/cold spell days are included in the average values. This obscures the comparison between different heat waves and cold spells because of differences in their length.

Accordingly, we used the regression models to predict mortality in the 30 days after the heat wave or cold spell by removing the mortality effects of the extreme heat or cold itself. This was performed by assuming that, in the absence of extreme temperatures, the average temperature during the heat wave or cold spell period could be estimated by linear interpolation between the day before and the day after the heat wave or cold spell, and by recalculating the average temperature in the heat wave or cold spell. We then used average temperatures, including the recalculated values, in the models to predict post-heat wave or post-cold spell mortality in absence of the extreme temperatures. To study the forward displacement of deaths caused by extreme temperatures, we compared the predicted mortality values in the absence of a heat wave or cold spell to the observed post-heat wave or cold spell mortality during different lag periods 
after the heat wave or cold spell in the month after the event. Mortality displacement could not be studied for the cold spell in winter 1978-1979 because this cold spell started before the beginning of our study period (1 January 1979) and the necessary mortality data was not available before that day.

Because age-specific mortality data is only available for the two latest cold spells, we did not perform an age-specific analysis of cold spell-induced forward displacement of mortality.

\subsubsection{The mortality-temperature relationship in the Netherlands}

The relationship between mortality and average temperature was V-like, with an optimum temperature value corresponding to the lowest point in the curve (Figures 6.3 and 6.4 ). This optimum value was $16.5^{\circ} \mathrm{C}$ for total mortality, cardiovascular mortality, respiratory mortality and mortality among those $\geq 65$ years of age, whereas for mortality due to malignant neoplasms and mortality in the younger age group, the optimum value was $15.5^{\circ} \mathrm{C}$ respectively $14.5^{\circ} \mathrm{C}$.

The reduction in scaled deviance of the null model (time trend only) is a measure of the explanatory power of the different models and, therefore, of the goodness of fit of the models. The explanatory power of the model for mortality due to all causes (including time trend, season, heat, cold, all lags heat, all lags cold) is relatively large; the reduction in scaled deviance of the model is $68 \%$. The models for death due to cardiovascular diseases (including season, heat, cold, all lags heat, all lags cold) and mortality in the older age group (including time trend, season, heat, cold, all lags heat, all lags cold) have the greatest explanatory power, with a reduction in scaled deviance in the null model of $75 \%$ and $72 \%$, respectively. These models are followed by the respiratory model (including time trend, season, heat, cold, all lags heat, all lags cold) with a reduction of $60 \%$. The explanatory power of the models for mortality due to malignant neoplasms (including time trend, season, heat, cold, all lags heat except lag-days 15-30, all lags cold except lag-days 15-30) and for mortality among those 0-64 years of age (including heat, cold, lag-days 7-14 heat, lag-days $7-14$ cold) is rather small, $41 \%$ and $25 \%$, respectively.

The season variables met both inclusion criteria in all models, except in the model for the younger age group. In this model, the season variables were not significant. It seems that the slope of the relationship between warmth and allcause mortality becomes steeper above a second turning point in the graph (approximately $22^{\circ} \mathrm{C}$ ), but adding a third temperature variable, extreme heat, did not lead to an increase in the explanatory power in any of the models.

Table 6.3 and Table 6.4 show the results of the adjusted regression models. For example, the $0.27 \%$ effect of cold for the lag-days $1-2$ in the model for all-cause mortality means that a $1^{\circ} \mathrm{C}$ increase in the average value of cold in the previous 
164 Chapter 6

2 days (i.e., a $1^{\circ} \mathrm{C}$ decrease in the average temperature below $16.5^{\circ} \mathrm{C}$ ) is associated with a $0.27 \%$ increase in mortality.

Table 6.3: Association between heat and daily mortality (due to different causes and in different age groups) controlled for the long-term mortality trend and season, the Netherlands, 1979-1997.

\begin{tabular}{|c|c|c|c|c|c|c|}
\hline & $\begin{array}{l}\text { Total } \\
\text { mortality }\end{array}$ & $\begin{array}{l}\text { Mortality } \\
\text { age } 0-64 \text { bc }\end{array}$ & $\begin{array}{l}\text { Mortality, } \\
\text { age } 65 \text { and } \\
\text { over }\end{array}$ & $\begin{array}{l}\text { Mortality } \\
\text { due to } \\
\text { malignant } \\
\text { neoplasms } \\
\text { d }\end{array}$ & $\begin{array}{l}\text { Cardio- } \\
\text { vascular } \\
\text { mortality }\end{array}$ & $\begin{array}{l}\text { Respira- } \\
\text { tory } \\
\text { mortality }\end{array}$ \\
\hline $\begin{array}{l}\text { Optimum } \\
\left({ }^{\circ} \mathrm{C}\right)\end{array}$ & 16.5 & 14.5 & 16.5 & 15.5 & 16.5 & 16.5 \\
\hline $\begin{array}{l}\text { Mean \# } \\
\text { deaths (std. } \\
\text { deviation) }\end{array}$ & $344(41)$ & $75(10)$ & $287(36)$ & $94(12)$ & $143(19)$ & $18(8)$ \\
\hline Heat $\mathrm{l}$ & & 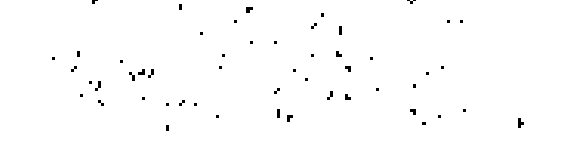 & & & & \\
\hline lag $=0$ & 1.59 & 0.98 & 1.51 & 1.34 & 1.42 & 2.43 \\
\hline lag $=1-2$ & 1.18 & - & 1.46 & 0.46 & 1.12 & 3.89 \\
\hline lag $=3-6$ & 0.41 & - & 1.11 & -0.79 & 0.19 & 4.38 \\
\hline lag $=7-14$ & -0.10 & -0.80 & 0.37 & -0.54 & -0.38 & 2.92 \\
\hline $\operatorname{lag}=15-30$ & -0.36 & - & -0.07 & - & -0.49 & -0.80 \\
\hline $\begin{array}{l}\text { aggregate } \\
\text { heat } \\
\text { relative } \mathrm{s}\end{array}$ & 2.72 & 0.18 & 4.38 & 0.47 & 1.86 & 12.82 \\
\hline $\begin{array}{l}\text { aggregate } \\
\text { heat } \\
\text { absolute h }\end{array}$ & 9.46 & 0.14 & 12.5 & 0.44 & 2.57 & 2.3 \\
\hline
\end{tabular}

Calculated with full model (including time trend, season, heat, cold, all lags heat, all lags cold).

Only 1988-1997 data.

Calculated with model without time trend, season, lags 1-2, lags 3-6 and lags 15-30.

Calculated with model without lags 15-30.

Calculated with model without time trend.

Change in mortality (\%) associated with a $1{ }^{\circ} \mathrm{C}$ increase in the average temperature above the optimum $16.5^{\circ} \mathrm{C}$. Estimated from regression analysis of the temperature-mortality relationship. Different adjusted models were used for the different causes of death.

The sum of the percental effects associated with the lag periods.

Calculated as the relative aggregated effect times the average daily number of deaths. This is according to Kunst et al. (1993). 
Table 6.4: Association between cold and daily mortality (due to different causes and in different age groups) controlled for the long-term mortality trend and season, the Netherlands, 1979-1997.

\begin{tabular}{|c|c|c|c|c|c|c|}
\hline & $\begin{array}{l}\text { Total } \\
\text { mortality } 2\end{array}$ & $\begin{array}{l}\text { Mortality, } \\
\text { age 0-64 bc }\end{array}$ & $\begin{array}{l}\text { Mortality, } \\
\text { age } 65 \text { and } \\
\text { over ab }\end{array}$ & $\begin{array}{l}\text { Mortality } \\
\text { due to } \\
\text { malignant } \\
\text { neoplasms } \\
\text { d }\end{array}$ & $\begin{array}{l}\text { Cardio- } \\
\text { vascular } \\
\text { mortality }\end{array}$ & $\begin{array}{l}\text { Respira- } \\
\text { tory } \\
\text { mortality a }\end{array}$ \\
\hline $\begin{array}{l}\text { Optimum } \\
\left({ }^{\circ} \mathrm{C}\right)\end{array}$ & 16.5 & 14.5 & 16.5 & 15.5 & 16.5 & 16.5 \\
\hline $\begin{array}{l}\text { Mean \# } \\
\text { deaths (std. } \\
\text { deviation) }\end{array}$ & $344(41)$ & $75(10)$ & $287(36)$ & $94(12)$ & $143(19)$ & $18(8)$ \\
\hline \multicolumn{7}{|l|}{ Cold } \\
\hline lag $=0$ & -0.31 & 0.02 & -0.37 & -0.45 & -0.07 & -0.66 \\
\hline $\operatorname{lag}=1-2$ & 0.27 & - & 0.30 & 0.20 & 0.39 & 0.15 \\
\hline $\operatorname{lag}=3-6$ & 0.38 & - & 0.39 & 0.32 & 0.33 & 0.57 \\
\hline $\operatorname{lag}=7-14$ & 0.56 & 0.48 & 0.79 & 0.15 & 0.61 & 2.04 \\
\hline lag $=15-30$ & 0.47 & - & 0.94 & - & 0.43 & 3.05 \\
\hline $\begin{array}{l}\text { aggregate } \\
\text { cold } \\
\text { relative }\end{array}$ & 1.37 & 0.5 & 2.05 & 0.22 & 1.69 & 5.15 \\
\hline $\begin{array}{l}\text { aggregate } \\
\text { cold } \\
\text { absolute h }\end{array}$ & 4.71 & 0.38 & 5.88 & 0.21 & 2.42 & 0.93 \\
\hline & \multicolumn{6}{|c|}{$\begin{array}{l}\text { Calculated with full model (including time trend, season, heat, cold, all lags heat, all lags } \\
\text { cold). }\end{array}$} \\
\hline Or & \multicolumn{6}{|c|}{ Only 1988-1997 data. } \\
\hline $\mathrm{Ca}$ & \multicolumn{6}{|c|}{$\begin{array}{l}\text { Calculated with model without time trend, season, lags } 1-2 \text {, lags } 3-6 \text { and lags } 15-30 \text {. } \\
\text { Calculated with model withour lags 15-30. }\end{array}$} \\
\hline $\mathrm{Ca}$ & \multicolumn{6}{|c|}{ Calculated with model without lags $15-30}$. \\
\hline $\mathrm{Ca}$ & \multicolumn{6}{|c|}{ Calculated with model without time trend. } \\
\hline $\begin{array}{l}\text { Ch } \\
\text { the } \\
\text { rel }\end{array}$ & \multicolumn{6}{|c|}{$\begin{array}{l}\text { Change in mortality }(\%) \text { associated with a } 1{ }^{\circ} \mathrm{C} \text { decrease in the average temperature below } \\
\text { the optimum } 16.5^{\circ} \mathrm{C} \text {. Estimated from regression analysis of the temperature-mortality } \\
\text { relationship. Different adjusted models were used for the different causes of death. }\end{array}$} \\
\hline Th & \multicolumn{6}{|c|}{ The sum of the percental effects associated with the lag periods. } \\
\hline $\begin{array}{l}\mathrm{Ca} \\
\mathrm{Th}\end{array}$ & \multicolumn{6}{|c|}{$\begin{array}{l}\text { Calculated as the relative aggregated effect times the average daily number of deaths. } \\
\text { This is according to Kunst et al. (1993). }\end{array}$} \\
\hline
\end{tabular}

The estimates for heat show an immediate and positive effect, in particular for respiratory diseases. There is an inverse relationship between heat and mortality in the longer lag periods before the index day. The compensatory effects are the smallest for respiratory disease mortality, and the relative aggregated effect is the largest for this kind of mortality. The compensatory effects are the largest for deaths due to malignant neoplasms; nearly threefourths of the effect within 3 days is compensated by a decrease in the number 
of deaths in the longer lag periods. In absolute terms, nearly $30 \%$ of all heatrelated deaths (9.46 deaths/day $/ 1^{\circ} \mathrm{C}$ heat during last month) are due to cardiovascular diseases (2.57 deaths $/$ day $/ 1^{\circ} \mathrm{C}$ heat during the last month) and nearly $25 \%$ is due to respiratory diseases $\left(2.3\right.$ deaths/day $/ 1^{\circ} \mathrm{C}$ heat during the last month) (Table 6.3).

In almost every lag period, there is positive relationship between cold and mortality (Table 6.4). The negative percentage effect of cold on mortality at lagday 0 is remarkable. The positive relationships between cold and mortality are relatively weak for malignant neoplasms and relatively strong for respiratory diseases. The latter is probably due to the strong positive effect of cold on respiratory mortality in the 7-30 days before the index. The relative effect on mortality due to cardiovascular disease is slightly larger than that for total mortality, but much weaker than for respiratory mortality. In absolute terms, about one-half of all cold-related deaths $\left(4.71\right.$ deaths/day $/ 1^{\circ} \mathrm{C}$ cold during the last month) are due to cardiovascular diseases $\left(2.42\right.$ deaths $/$ day $/ 1^{\circ} \mathrm{C}$ cold during the last month).

\subsubsection{Excess mortality during heat waves and cold spells}

Tables 6.5 and 6.6 show the results of the analyses of excess mortality during the heat waves and cold spells in the study period, based on the 31-day moving average for the same year as well the 31-day moving average of the 2 years before the heat wave or cold spell. The 31-day moving average for the same year contains the possible excess mortality during the heat wave or cold spell days and is, therefore, expected to be larger than the 31-day moving average for years without extreme heat or cold during the same period. The results presented below are based on the calculations using the 31-day moving average of the 2 years before the heat wave or cold spell.

Table 6.5 shows significant excess total mortality during all of the heat waves studied, particularly mortality due to respiratory causes. The average excess in all-cause mortality during these heat waves was $12.1 \%$ or 39.8 deaths/heat wave day. The largest excess is seen for the 1994 heat wave (24\%), which was also the longest heat wave in the study period (13 days). The excess respiratory mortality of $120 \%$ during the 1994 heat wave is noteworthy. This is also significant in the 1982, 1983, and 1990 heat waves, and excess cardiovascular mortality is significant during the 1983, 1990, 1994, and 1995 heat waves. Mortality caused by malignant neoplasms increased significantly during the 1983, 1994, and 1995 heat waves. The total excess mortality is largely attributable to increases in mortality in the $\geq 65$ age group, whereas the heat had little effect among those younger than 65 . In general, the health status of older persons is more compromised than the health of younger people (Chan et al., 2001). For example, artheromatous arteries are much more common among 
older people, which exacerbates the hematologic changes induced by extreme temperatures (Keatinge \& Donaldson, 1995). The elderly are also at greater risk due to a reduced thermoregulatory response and less sensitive thermal perception (Keatinge \& Donaldson, 1995).

Table 6.5: Excess mortality during heat waves by selected causes of death and selected age groups.

\begin{tabular}{|c|c|c|c|}
\hline $\begin{array}{l}\text { Yeat } \\
\text { summer }\end{array}$ & Mortality & $\begin{array}{l}\text { Compared with } 31 \text { dma for } \\
\text { the preceding two years, } \\
\text { number }[95 \% \text { ch } 1 \\
\text { percentage (excess per day) }\end{array}$ & $\begin{array}{l}\text { Compared with } 31-\mathrm{dma} \text { for } \\
\text { the same year } \\
\text { number }[95 \% \mathrm{ch}) \text {, } \\
\text { percentage, (excess per day) }\end{array}$ \\
\hline \multirow[t]{4}{*}{1982} & Total & $184[90 ; 278] 8.7 \%(26.3)$ & $159[66 ; 253] 7.5 \%(22.7)$ \\
\hline & Malignant causes & $42[-7 ; 92] 7.2 \%(6.0)$ & $34[-16 ; 83] 5.6 \% \quad(4.9)$ \\
\hline & Cardiovasc. causes & $21[-39 ; 81] 2.3 \%(3.0)$ & $38[-22 ; 98] 4.2 \%(5.4)$ \\
\hline & Respiratory causes & $32[9 ; 55] 30.7 \% 4.6)$ & $14[-10 ; 36] 10.9 \%(2.0)$ \\
\hline \multirow[t]{4}{*}{1983} & Total & $267[158 ; 375] 9.6 \%(29.7)$ & $134[26 ; 243] 4.6 \%(14.9)$ \\
\hline & Malignant causes & $69[12 ; 127] 8.8 \%(7.7)$ & $42[-15 ; 100] 5.2 \%(4.7)$ \\
\hline & Cardiovasc. causes & $89[18 ; 159] 7.3 \%(9.9)$ & $72[1 ; 143] 5.9 \%(8.0)$ \\
\hline & Respiratory causes & $26[1 ; 51] 19.5 \%(2.9)$ & $9[-16 ; 34] 6.2 \%(1.0)$ \\
\hline \multirow[t]{6}{*}{1990} & Total & $339[223 ; 457] 10.6 \% \quad(33.9)$ & $182[66 ; 299] 5.4 \% \quad(18.2)$ \\
\hline & Aged 0-64 years & $39[-15 ; 93] 5.4 \% \quad(3.9)$ & $49[-5 ; 104] 6.8 \%(4.9)$ \\
\hline & Aged $\geq 65$ years & $301[198 ; 404] 12.2 \%(30.1)$ & $133[30 ; 236] 5.0 \%(13.3)$ \\
\hline & Malignant causes & $8[-52 ; 69] 0.9 \%(0.8)$ & $28[-32 ; 89] 3.0 \%(2.8)$ \\
\hline & Cardiovasc. causes & $119[46 ; 191] 9.5 \%(11.9)$ & $74[1 ; 146] 5.7 \%(7.4)$ \\
\hline & Respiratory causes & $61[34 ; 87] 47.1 \%(6.1)$ & $19[-8 ; 56] 11.1 \%(1.9)$ \\
\hline \multirow[t]{6}{*}{1994} & Total & $1057[913 ; 1201] 24.4 \% \quad(81.3)$ & $240[96 ; 384] 4.7 \%(18.4)$ \\
\hline & Age 0-64 years & $15[-46 ; 75] 1.6 \%(1.1)$ & $1[-59 ; 61] 0.1 \% \quad(0.1)$ \\
\hline & Aged $\geq 65$ years & $1043[911 ; 1173] 30.7 \%(80.3)$ & $239[107 ; 370] 5.7 \%(18.4)$ \\
\hline & Malignant causes & $179[105 ; 253] 14.2 \%(13.8)$ & $99[25 ; 174] 7.5 \%(7.6)$ \\
\hline & Cardiovasc. causes & $244[159 ; 328] 15.1 \%(18.8)$ & $39[-46 ; 123] 2.1 \%(3.0)$ \\
\hline & Respiratory causes & $247[205 ; 289] 120 \%(19)$ & $37[-5 ; 78] 8.8 \%(2.8)$ \\
\hline \multirow[t]{6}{*}{1995} & Total & $236[141 ; 332] 11.0 \%(39.3)$ & $224[128 ; 320] 10.3 \%(37.3)$ \\
\hline & Age $0-64$ years & $23[-19 ; 64] 5.3 \%(3.8)$ & $12[-30 ; 53] 2.7 \%(2.0)$ \\
\hline & Aged $\geq 65$ years & $214[127 ; 300] 12.4 \%(35.7)$ & $212[126 ; 298] 12.3 \%(35.3)$ \\
\hline & Malignant causes & $60[10 ; 110] 10.1 \%(10.0)$ & $61[11 ; 111] 10.3 \%(10.2)$ \\
\hline & Cardiovasc. causes & $81[24 ; 139] 10.5 \%(13.5)$ & $69[11 ; 126] 8.7 \%(11.5)$ \\
\hline & Respiratory causes & $13[-11 ; 38] 9.4 \%(2.2)$ & $20[-4 ; 45] 15.1 \%(3.3)$ \\
\hline \multirow[t]{6}{*}{1997} & Total & $256[142 ; 371] 8.2 \% \quad(28.4)$ & $47[-67 ; 161] 1.4 \%(5.2)$ \\
\hline & Age $0-64$ years & $26[-25 ; 78] 4.0 \%(2.9)$ & $28[-23 ; 80] 4.3 \%(3.1)$ \\
\hline & Aged $\geq 65$ years & $230[128 ; 332] 9.3 \%(25.6)$ & $18[-84 ; 120] 0.7 \%(2.0)$ \\
\hline & Malignant causes & $47[-14 ; 107] 5.1 \%(5.2)$ & $29[-32 ; 90] 3.1 \%(3.2)$ \\
\hline & Cardiovasc. causes & $50[-17 ; 117] 4.5 \%(5.6)$ & $-6 \cdot[-73 ; 60]-0.5 \%(-0.7)$ \\
\hline & Respiratory causes & $16[-1 ; 55] 15.5 \%(1.8)$ & $5[-23 ; 33] 2.4 \%(0.6)$ \\
\hline
\end{tabular}


Table 6.6: Excess mortality during cold spells by selected causes of death and selected age groups.

\begin{tabular}{|c|c|c|c|}
\hline $\begin{array}{l}\text { Year/ } \\
\text { winter }\end{array}$ & Mortality & $\begin{array}{l}\text { Compared with } 31 \text {-dma* for } \\
\text { the preceding two years, } \\
\text { number ( } 95 \% \text { c.i.), } \\
\text { percentage, (excess per day) }\end{array}$ & $\begin{array}{l}\text { Compared with } 31 \text {-dma* for } \\
\text { the same year } \\
\text { number }[95 \% \text { c.i.], } \\
\text { percentage, (excess per day) }\end{array}$ \\
\hline 1984- & Total & $598[439 ; 756] 10.1 \%(35.2)$ & $116[-43 ; 275] 1.8 \%(6.8)$ \\
\hline 1985 & $\begin{array}{l}\text { Malignant causes } \\
\text { Cardiovasc. causes } \\
\text { Respiratory causes }\end{array}$ & $\begin{array}{l}73[-6 ; 152] 4.7 \%(4.3) \\
365[265 ; 474] 13.4 \%(21.5) \\
19[-19 ; 57] 5.3 \%(1.1)\end{array}$ & $\begin{array}{l}37[-41 ; 116] 2.4 \%(2.2) \\
59[-50 ; 168] 1.9 \%(35) \\
-5[-43 ; 33]-1.3 \%(-0.3)\end{array}$ \\
\hline $\begin{array}{l}1985- \\
1986\end{array}$ & $\begin{array}{l}\text { Total } \\
\text { Malignant causes } \\
\text { Cardiovasc. causes } \\
\text { Respiratory causes }\end{array}$ & $\begin{array}{l}1736[1558 ; 1913] 26.8 \% \\
(102.1) \\
170[86 ; 253] 10.3 \%(10.0) \\
683[565 ; 802] 23.0 \%(40.2) \\
462[405 ; 519] 117.2 \%(27.2)\end{array}$ & $\begin{array}{l}306[129 ; 1184] 3.9 \%(18.0) \\
17[-67 ; 100] 0.9 \%(1.0) \\
170[51 ; 288] 4.9 \%(10.0) \\
69[11 ; 126] 8.7 \%(4.1)\end{array}$ \\
\hline 1990- & Total & $403[38 ; 276] 12.3 \%(44.8)$ & $157[38 ; 276] 4.5 \%(17.4)$ \\
\hline 1991 & $\begin{array}{l}\text { Aged 0-64 years } \\
\text { Aged } \geq 65 \text { years } \\
\text { Malignant causes } \\
\text { Cardiovasc. causes } \\
\text { Respiratory causes }\end{array}$ & $\begin{array}{l}103[48 ; 158] 15.0 \%(11.4) \\
301[195 ; 406] 11.6 \%(33.4) \\
25[-33 ; 84] 2.9 \%(2.8) \\
244[166 ; 322] 18.1 \%(27.1) \\
16[-12 ; 43] 8.7 \%(1.8)\end{array}$ & $\begin{array}{l}85[30 ; 140] 12.1 \%(9.4) \\
72[-34 ; 177] 2.5 \%(8.0) \\
-2[-60 ; 56]-0.2 \%(-0.2) \\
109[31 ; 187] 7.4 \%(12.1) \\
-13[-41 ; 14]-6.4 \%(-1.4)\end{array}$ \\
\hline 1996- & Total & $137[-32 ; 306] 1.9 \%(8.1)$ & $55[-114 ; 224] 0,7 \%(3.2)$ \\
\hline 1997 & $\begin{array}{l}\text { Age } 0-64 \text { years } \\
\text { Aged } \geq 65 \text { years } \\
\text { Malignant causes } \\
\text { Cardiovasc. causes } \\
\text { Respiratory causes }\end{array}$ & $\begin{array}{l}-20[-92 ; 52]-1.5 \%(-1.2) \\
157[4 ; 310] 2.6 \%(9.2) \\
45[-39 ; 129] 2.5 \%(2.6) \\
18[-86 ; 123] 0.7 \%(1.1) \\
-15[-63 ; 33]-2.4 \%(-0.9)\end{array}$ & $\begin{array}{l}-9[-81 ; 62]-0,7 \%(-0.5) \\
64[-89 ; 218] 1,1 \%(3.8) \\
31[-53 ; 115] 1,7 \%(1.8) \\
-4[-108 ; 100]-0,1 \%(-0.2) \\
-3[-50: 45]-0,4 \%(-0.2)\end{array}$ \\
\hline
\end{tabular}

There was significant excess mortality due to all causes in the first three cold spells (Table 6.6). The lack in excess mortality during the cold spell in winter 1996-1997 is noteworthy. This could be explained by increased influenza mortality during the last few weeks of 1995, which resulted in a higher 31-day moving average of the two preceding years of the 1996-1997 cold spell and, as a consequence, to an underestimation of the excess mortality during this cold spell. The only significant increase in mortality during this cold spell was among those $\geq 65$ years of age.

The average excess in all-cause mortality during all of the studied cold spells was $12.8 \%$, or 47.6 deaths/cold spell day. The most striking increase in mortality occurred in the winter of 1985-1986. Mortality increased significantly for all the selected causes and the percentage of excess mortality was highest in this period, compared with the other cold spells. Excess mortality due to respiratory causes during this cold spell is more than $117 \%$. An influenza epidemic in the same period (February-March 1986) (Mackenbach et al., 1992) is probably responsible for this sharp increase. In the 1984-1985, 1990-1991, and 1996-1997 
cold spells, there was no significant excess respiratory mortality. In the same three cold spells, the mortality caused by neoplasms also did not increase significantly. Cardiovascular mortality increased significantly during the cold spells in the winters of 1984-1985, 1985-1986, and 1990-1991. During the first two cold spells, the percentage excess mortality was the highest for cardiovascular mortality compared to the other causes during the same cold spell. Table 6.6 shows that in both cold spells for which data for different age groups are available (winter 1990-1991 and winter 1996-1997), the excess mortality in those $\geq 65$ years of age is larger than that in the younger age group. A difficulty arises when one or both of the 2 years before the heat wave or cold spell year also contains a period with extreme high or low temperatures; these can influence mortality and, subsequently, the 31-day moving average of the 2 years before the heat wave or cold spell. This would lead to an underestimation of the excess mortality during the heat wave or cold spell. For example, during winter 1984-1985 a cold spell occurred during 4 January 1985-20 January 1985, while the mortality during 12 February 1985-27 February 1985 was used to determine part of the 31-day moving average of the 2 years before the cold spell in 1985-1986. It is possible that the cold spell in January 1985 had an influence on the mortality during 12 February 1985-27 February 1985, despite an intervening 3-week period. Such interference will result in a larger 31-day moving average for the 2 years before the cold spell in winter 1985-1986. However, during the heat waves $(1983,1995,1997)$ and cold spell (1985-1986) for which this could be a problem, the excess mortality is still significant.

Tables 6.7 and 6.8 show the results of the forward displacement of deaths due to the heat waves and cold spells. The mortality from malignant neoplasms and mortality in the younger age group was excluded from this analysis because of the lack of explanatory power of the regression models. Table 6.7 shows a mortality deficit (all causes) in the longer lag period after the 1983, 1990, 1995, and 1997 heat waves. In the other two heat waves (1982 and 1994), we found no mortality deficit. For the selected mortality groups, the results are inconclusive: some heat waves show a decline in mortality in the following period, while others cause an increase in mortality (some of which is significant).

Excess all-cause mortality, as well as that caused by cardiovascular and respiratory diseases, seems to continue during the whole month after the cold spells. A clear decline in mortality, which would suggest that cold has a harvesting effect, is not evident. Thus, Table 6.8 shows that the cold spells studied probably did not lead to any considerable forward displacement of deaths among those who would have died in the short term anyway. 
Table 6.7: Difference between predicted mortality in the absence of the extreme heat and the observed mortality, for a range of time intervals after the heat waves.

\begin{tabular}{|c|c|c|c|c|c|}
\hline \multicolumn{2}{|c|}{ Cause \& heat wave } & $\begin{array}{l}\text { Excess }{ }^{2} \\
\operatorname{lag}=1-2^{b}\end{array}$ & $\begin{array}{l}\text { Excess }{ }^{2} \\
\text { Lag }=3-6^{c}\end{array}$ & $\begin{array}{l}\text { Excess } \\
\text { lag }=7-14^{d}\end{array}$ & $\begin{array}{l}\text { Excess a } \\
\text { lag }=15-30\end{array}$ \\
\hline \multicolumn{6}{|c|}{ Total mortality, all causes } \\
\hline \multicolumn{2}{|c|}{1982} & 27 & 25 & 4 & $112^{* * 1}$ \\
\hline \multicolumn{2}{|l|}{1983} & 36 & 35 & -45 & $-289^{* * 3}$ \\
\hline \multicolumn{2}{|l|}{1990} & $142^{* * *}$ & -7 & -58 & -103 \\
\hline \multicolumn{2}{|l|}{1994} & 142 & 70 & $103^{* * 2}$ & $275^{* * 2}$ \\
\hline \multicolumn{2}{|l|}{1995} & 30 & 22 & -90 & $-278^{* * 2}$ \\
\hline \multicolumn{2}{|l|}{1997} & 117 & $168^{* * *}$ & -1 & $-177^{* * * 2}$ \\
\hline \multicolumn{6}{|c|}{ Cardiovascular diseases } \\
\hline \multicolumn{2}{|c|}{1982} & -2 & $37^{* *}$ & -4 & 66 \\
\hline \multicolumn{2}{|l|}{1983} & 11 & 1 & 11 & $-139 * * * 3$ \\
\hline \multicolumn{2}{|l|}{1990} & 59 & -13 & $-62 * * 2$ & -63 \\
\hline \multicolumn{2}{|l|}{1994} & 65 & 5 & -2 & $160^{* * *}$ \\
\hline \multicolumn{2}{|l|}{1995} & 11 & -13 & -15 & -26 \\
\hline \multicolumn{2}{|c|}{1997} & 31 & $37^{*}$ & -7 & -19 \\
\hline \multicolumn{6}{|c|}{ Respiratory díseases } \\
\hline \multicolumn{2}{|c|}{1982} & 23 & 14 & $38^{* * * 3}$ & $23^{* * 2}$ \\
\hline \multicolumn{2}{|l|}{1983} & 9 & 13 & -6 & $40^{* * 2}$ \\
\hline \multicolumn{2}{|l|}{1990} & 31 & 7 & 7 & -16 \\
\hline \multicolumn{2}{|l|}{1994} & $47^{*}$ & $40^{* * *}$ & $70^{* * * 3}$ & $43^{* * 2}$ \\
\hline \multicolumn{2}{|l|}{1995} & 20 & $24^{*}$ & 15 & 12 \\
\hline \multicolumn{2}{|c|}{1997} & 20 & $39^{*}$ & $41^{*}$ & 12 \\
\hline \multicolumn{6}{|c|}{ Age 65 and over } \\
\hline \multicolumn{2}{|c|}{1990} & $150^{* * *}$ & 15 & -73 & -78 \\
\hline \multicolumn{2}{|l|}{1994} & 163 & $113^{* *}$ & $118^{* * 2}$ & $403^{* * * 3}$ \\
\hline \multicolumn{2}{|l|}{1995} & 22 & 10 & -8 & $-169 * 2$ \\
\hline \multicolumn{2}{|l|}{1997} & $133^{*}$ & $233^{* * *}$ & $134^{* *}$ & 64 \\
\hline a & $\begin{array}{l}\text { excess }=o b \\
\text { extreme war }\end{array}$ & $\begin{array}{l}\text { number of } \\
\text { eratures ( }\end{array}$ & minus $p$ & number $c$ & ths in abse \\
\hline b & day $1-2$ after & of heat $w$ & & & \\
\hline c & day 3-6 after & of heat $w$ & & & \\
\hline d & day 7-14 afte & $g$ of heat $n$ & & & \\
\hline e & day $15-30$ af & ng of heat & & & \\
\hline * & t-test, $p<0.1$ & & & & \\
\hline ** & t-test, $\mathrm{p}<0.0$ & & & & \\
\hline *** & t-test, $p<0.0$ & & & & \\
\hline 1 & sign-rank te & & & & \\
\hline 2 & sign-rank te & & & & \\
\hline 3 & sign-rank te & & & & \\
\hline
\end{tabular}


Table 6.8: Difference between predicted mortality in the absence of the extreme cold and the observed mortality, for a range of time intervals after the cold spells.

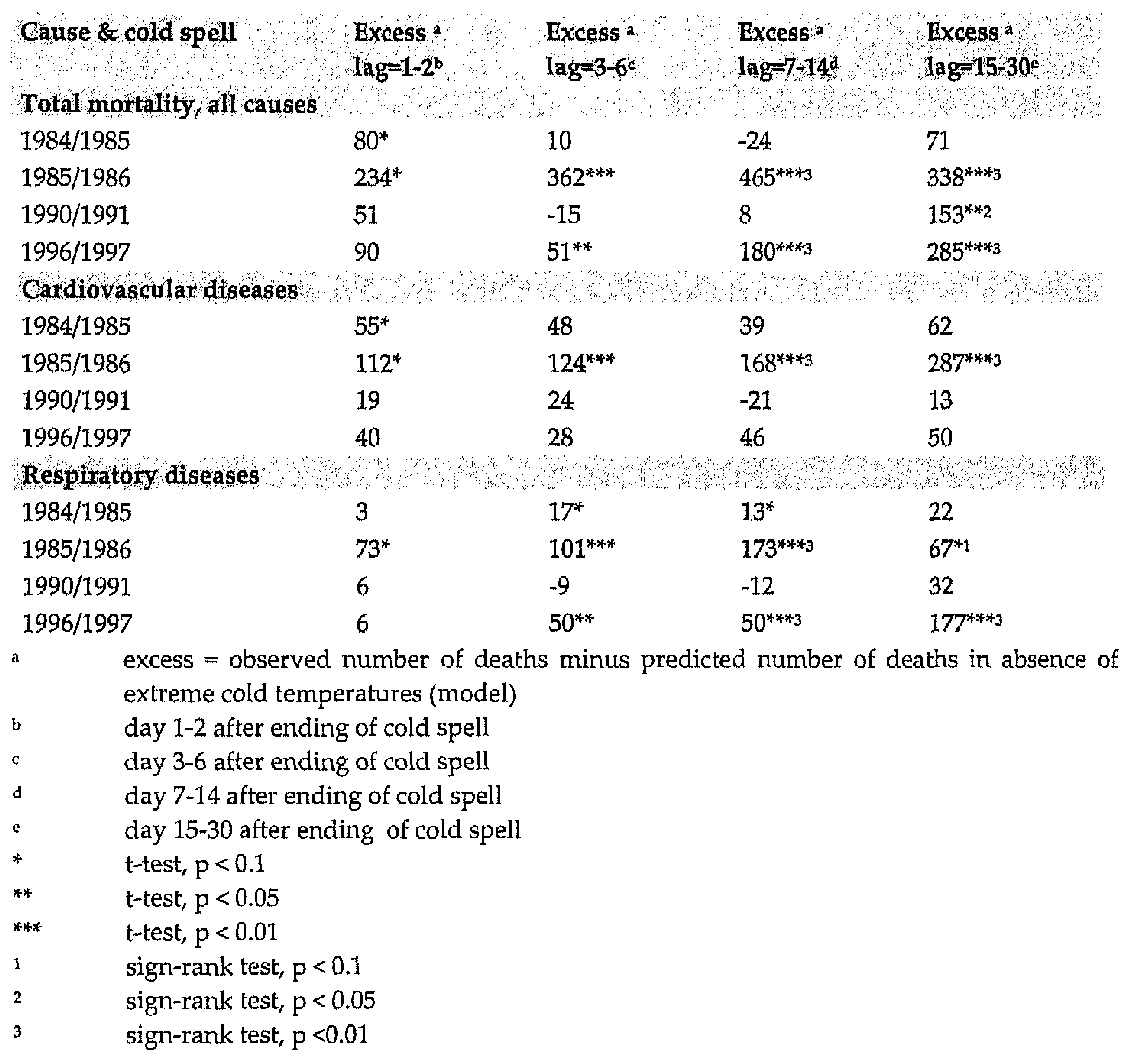

\subsubsection{Discussion and conclusion}

Our model results show an immedir selected categories (Table 6.3). compensatory effects, which su occurs in persons whose displacement of deaths).

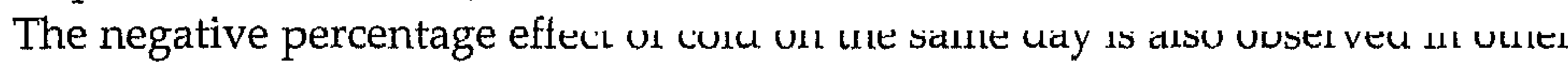
studies (Ballester et al., 1997; Kunst et al., 1993), but this has not been adequately explained. Furthermore, our model showed a lack of compensatory effects on mortality after exposure to cold temperatures (Table 6.4). This 
supports the findings of Kunst et al. (1993) in their study of temperature and mortality in the Netherlands. It has been suggested that the effects of cold could be influenced by influenza and influenza-like conditions during the winter (Ballester et al., 1997; Kunst et al., 1993). Kunst et al. (1993) found that influenza incidence in their regression models could only partly explain the effect of cold temperatures and that this was only the case for the effect of cold temperatures in the previous 7-30 days. In the same study, more than one-half of the unexplained mortality occurred within the first week, which strengthens the hypothesis that the relation between cold weather and mortality is largely attributable to the direct effects of cold. The significant increases in mortality found during the periods of extreme cold temperatures that we studied provide a further indication of the likelihood of this hypothesis being correct.

We found significant excess total mortality $(8.7-24.4 \%)$ during the six heat waves in the study period (Table 6.5). The increase in all-cause mortality was highest during the 1994 heat wave, which was also the longest heat wave. On average, the extremely high temperatures resulted in an average of 38.9 excess deaths per day or an excess mortality of $12.8 \%$. This latter percentage is in line with other studies (McMichael \& Kovats, 1998; Rooney et al., 1998; Sartor et al., 1995). The Dutch heat wave of August 2003 lasted about 2 weeks. Compared with the mortality rate during a normal August (with a mean temperature of about $22^{\circ} \mathrm{C}$ ), Statistics Netherlands concluded that about 400-500 extra deaths occurred due to the extreme heat in this period (de Beer \& Harmsen, 2003). Heat waves seem to have the potential to affect mortality from all of the selected causes. Relative excess mortality is generally largest for respiratory diseases, particularly during the 1994 heat wave, when it reached $120 \%$. This can probably be explained by contributing factors such as the high levels of photochemical air pollution (van Doesburg, 1995). The heat-induced mortality increases mainly occur among those $\geq 65$ years of age.

Earlier studies (Alberdi et al., 1998; Eng \& Mercer, 1998; Kunst et al., 1993; Rooney et al., 1998) supported the hypotheses that high temperatures result in the forward displacement of deaths. Our results relating to the heat-induced forward displacement of deaths are inconclusive (Table 6.7). Some heat waves show a decline in mortality in the longer lag periods after the extreme heat, which suggests that heat has a harvesting effect, whereas others do not show this decline in the number of deaths. It is possible that contributing factors (for example, earlier episodes of relatively warm weather) influence the potential of heat waves not merely to cause the forward displacement of deaths but to make a substantial contribution to overall lost lifetime.

We found significant excess all-cause mortality in all of the cold spells studied, except during the winter of 1996-1997 (Table 6.6). During the cold spells that showed a significant increase in the total number of deaths, the excess mortality was between $10.1 \%$ and $26.8 \%$. The most striking increase in mortality occurred in the winter of 1985-1986 (excess mortality due to respiratory causes is even 
$117.2 \%$ ), probably due to an influenza epidemic in the same period (Mackenbach et al., 1992).

Our results show that in the absence of such an influenza epidemic, respiratory mortality does not increase during a period of extreme cold. It is possible, however, that the respiratory effects of low temperatures influence mortality after the end of the cold spells. Respiratory mortality peaks after mortality due to cardiovascular causes, suggesting different lag times in the effect of cold (Alberdi et al., 1998; Donaldson \& Keatinge, 1997; Eurowinter Group, 1997; Keatinge \& Donaldson, 1995; Kunst et al., 1991; Kunst et al., 1993). Respiratory cross-infection may explain the delayed effect of cold (Donaldson \& Keatinge, 1997; Eurowinter Group, 1997; Kunst et al., 1991; Kunst et al., 1993; Mackenbach et al., 1992). In the Netherlands, the occurrence of influenza and influenza-like conditions have been found to be strongly correlated with low temperatures, in particular 14 days after cold weather (Kunst et al., 1991). Cardiovascular mortality increased during cold spells, confirming the rapid effect of cold on this type of mortality, except during the cold spell in winter 1996-1997. The absence of an increase in cancer mortality during cold spells except in February 1986 suggests that unusually low temperature has no impact. Kunst et al. (1991) also stated that the relationship between mortality due to malignant diseases and cold is rather weak. The excess mortality is much greater among the older age group than in the younger group. As discussed earlier, the $\geq 65$-year-old group would be expected to be the most sensitive to extreme temperatures.

We did not observe a decrease in mortality after the cold spells (Table 6.8). This suggests that extreme cold does not lead to any mortality displacement. This contrasts with our findings and those of others relating to mortality after heat waves (Alberdi et al., 1998; Eng \& Mercer, 1998; Kunst et al., 1993; Rooney et al., 1998). There is a significant and relatively large excess of mortality from all causes, as well as from cardiovascular and respiratory diseases during a whole month after the 1985-1986 cold spell. This is probably due to the influenza epidemic in this period (Mackenbach et al., 1992), because our regression models did not correct for the incidence of influenza. Furthermore, the relatively high excess mortality due to respiratory diseases after the 1996-1997 cold spell is probably due to the increase in influenza incidence during the same period.

When considering our conclusions regarding the separate heat waves and cold spells, it is important to bear in mind that this study is based on a very small number of heat waves and cold spells. Also, these results depend on the definition of heat waves and cold spells used.

One assumption was that the 31-day moving average method produces an accurate approximation of mortality in the absence of extreme temperatures. If the daily mortality numbers used for the two preceding years differ from normal values (i.e., due to high influenza incidence, very high or low 
temperatures), the 31-day moving average for this period can result in a biased approximation of mortality in the absence of a cold spell.

We assumed that the models were able to accurately predict mortality in the absence of heat waves and cold spells. The models for total mortality and respiratory and cardiovascular mortality, however, cannot explain $32 \%, 40 \%$, and $25 \%$, respectively, of the variation in daily mortality. Also, we were not able to correct for the effects of influenza in our models.

Only the effect of temperature, controlled for time trend and seasonal influence, was determined. However, it is conceivable that the influences of other weather components should also be included (e.g., atmospheric pressure, fronts, number of hours of sunshine per day, relative humidity, wind speed and air pollution) (Kunst et al., 1993; Larsen, 1990). The influence of relative humidity and wind speed has been observed (Kunst et al., 1993; Saez et al., 1995), whereas controlling for $\mathrm{SO}_{2}$ density did not alter the relationship between mortality and temperature in the Netherlands (Kunst et al., 1993). However, Fischer et al. (2004) concluded that part of the excess mortality during heat waves might be attributable to increased levels of air pollution (SMOG) during hot days. The increased mortality probably also implies an increase in morbidity, the size of which is at least proportional to that of the increased mortality and probably greater (Bresser et al., 2005).

The formulation of health policy could benefit from the results of research on the association between temperature and mortality (Ballester et al., 1997). Where substantial resources are dedicated to the treatment of diseases (Donaldson \& Keatinge, 1997), it may be appropriate to study prevention of these diseases by reducing the effects of temperature, especially among those at high risk. Bearing in mind that cold temperatures could be responsible for a substantial amount of lost lifetime, health policy designed to prevent the adverse effects of cold spells should be considered. For example, during the winter of 1986-1987 a media campaign in the United Kingdom, which advised elderly people to avoid outdoor exposure, was accompanied by a dramatic fall in winter deaths compared to the numbers predicted by the trend over the previous decade (Keatinge \& Donaldson, 1995). The EUROheat project (WHO, 2007) looks at the effects of public health responses (e.g., heat-health warning systems) in Europe.

\subsection{CLIMATE CHANGE, TEMPERATURE CHANGE AND MORTALITY}

The IPCC (2007a) recently stated that further research is needed to understand how the balance of heat-related and cold-related mortality could change under different climate projections. In an earlier study into the mortality effects of climate change, Martens and Huynen (2001) estimated future changes in mortality rates by combining observed mortality risks accompanying 
temperature change (see section 6.1 ) with the projected changes in monthly temperature estimated by nine General Circulation Models (GCMs) for the year 2050 under the IPCC 550 ppm stabilisation scenario (e.g., approximate doubling of the pre-industrial level of greenhouse gases). Their results are based on the assumptions that other relevant factors remain unchanged. Their findings showed that for The Netherlands the continuing decreasing trend in winter mortality is more pronounced than the increasing excess summer mortality rates. Net yearly decreases in mortality rates varied between 0.9 and $1.6 \%$ for cardiovascular mortality, between 1.6 and $3.9 \%$ for respiratory mortality, and between 0.6 and $1.1 \%$ for total mortality. The same trend was seen in scenarios where the GHG concentration stabilised around $350 \mathrm{ppm}$ or $750 \mathrm{ppm}$.

In this section, we assess the annual changes in the proportion temperaturerelated deaths attributable to climate changes, using recent estimates of daily temperatures in 2050 for the Netherlands. As the mortality due to malignant neoplasms and the mortality in the younger aged group (0-64 years) are less sensitive to temperature, these health outcomes have been excluded from this analysis. Due to the difficulties regarding the modelling of future heat waves and cold spells under various climate change scenarios (IPCC, 2007b; Stott et al., 2004), our study did not specifically look at the climate change-induced changes in mortality during episodes of extreme heat or cold.

\subsubsection{Methods}

\section{Climate change scenarios}

Projecting future climate change is subject to many uncertainties, such as those regarding the development of anthropogenic activities (and related emissions of greenhouse gases and changes in land use), and our limited understanding of the complex climate system (its inherent internal variability and its response to changes in concentrations of greenhouse gasses and land use changes). A means of dealing with such uncertainty is the construction of a set of climate scenarios. Climate scenarios are relevant, plausible and internally consistent pictures of how the climate may look like in the future (IPCC, 2001b).

In 2006, the KNMI formulated new climate change scenarios for the Netherlands (KNMI'06 scenarios) (van den Hurk et al., 2006). The selection of these new scenarios was based, among others, on the fact that future climate change in the Netherlands will mainly depend on the global temperature rise as well as on changes in the air circulation patterns in Western Europe (and the related changes in the wind). To be able to deal with the uncertainties in future climate change, four climate scenarios from the broad range of possible futures were selected. The KMMI considers it most likely that the Dutch climate will develop between these four 'corner points' (Table 6.9). An elaborate description with all relevant sources and references to the scientific literature can be found 
in the KNMI-publication 'KNMI climate change scenarios 2006 for the Netherlands' (van den Hurk et al., 2006).

In the $\mathrm{KNMI}^{\prime} 06$ scenarios, the anticipated changes in the climatological target period are described relative to a climatological baseline period around 1990. The seasonal mean temperature is derived from a combination of global and regional climate models. Table 6.10 describes the changes in average temperature in the Netherlands around 2050 compared to the baseline year 1990. The projected Dutch temperature rise deviates from the global temperature rise, especially in the scenarios with changes in air circulation. In these scenarios ( $\mathrm{G}+$ and $\mathrm{W}+$ ), the increase in the annual average temperature is higher compared to those scenarios without a change in air circulation $(G$ and W). Also, the increase in average temperature in $\mathrm{G}+$ and $\mathrm{W}+$ is higher in the summer months compared to the winter period.

Table 6.9: The KNMI'o6 scenarios for the Netherlands: basic assumptions regarding the temperature rise on earth and changes in the air circulation patterns in Western Europe (van den Hurk et al., 2006).

\begin{tabular}{|c|c|c|}
\hline Scenario & $\begin{array}{l}\text { Average } \\
\text { temperature rise } \\
\text { on earth } \\
\text { (baseline }=1990 \text { ) }\end{array}$ & Air circulation patterns in Western Europe \\
\hline $\mathrm{G}^{*}$ & $1^{\circ} \mathrm{C}$ in 2050 & No change \\
\hline Moderate & $2^{\circ} \mathrm{C}$ in 2100 & \\
\hline $\mathrm{G}+$ & $1^{\circ} \mathrm{C}$ in 2050 & Change in air circulation patterns in Western Europe \\
\hline Moderate + & $2^{\circ} \mathrm{C}$ in 2100 & $\begin{array}{l}\text { - milder and wetter winters due to more westerly winds } \\
\text { - warmer and dryer summers due to more easterly winds }\end{array}$ \\
\hline W & $2^{\circ} \mathrm{C}$ in 2050 & No change \\
\hline Warm & $4^{\circ} \mathrm{C}$ in 2100 & \\
\hline W+ & $2^{\circ} \mathrm{C}$ in 2050 & Change in air circulation patterns in Western Europe \\
\hline Warm + & $4^{\circ} \mathrm{C}$ in 2100 & $\begin{array}{l}\text { - milder and wetter winters due to more westerly winds } \\
\text { - warmer and dryer summers due to more easterly winds }\end{array}$ \\
\hline
\end{tabular}

A transformation of historic observations translates the climate change signal derived from the models into future time series at the different Dutch stations. This study used the observed and transformed time series of average temperature at the De Bilt station around 1990 (1976 to 2005) and 2050 (20352065). 
Table 6.10: Changes in average temperature in De Bilt (the Netherlands) in 2050, compared to the baseline year 1990, according to the four KNMI'06 climate scenarios (KNMI, 2007; van den Hurk et al., 2006).

$\begin{array}{llc}\text { Average temperature in summer (June, July, August) } & \text { Scenario } & \mathbf{2 0 5 0} \\ & \mathrm{G} & 0.9 \\ & \mathrm{G} & 1.4 \\ & \mathrm{~W} & 1.8 \\ \text { Average temperature in winter (December, January, February) } & \text { W+ } & 2.8 \\ & \mathrm{G} & 0.9 \\ & \mathrm{G}+ & 1.2 \\ & \mathrm{~W} & 1.8 \\ \text { Annual average temperature** } & \mathrm{W}+ & 2.4 \\ & \mathrm{G} & 0.9 \\ & \mathrm{G}+ & 1.3 \\ \text { * Seasonal mean temperature derived from a combination of global and regional climate models. } \\ \text { ** Based on transformed time series. } & \mathrm{W} & 1.8\end{array}$

Future exposure-response relationship between temperature and mortality

In section 6.1, we described the daily numbers of deaths due to all causes and to selected causes in relation to daily average temperature in the Netherlands, 1979-1997. We found that for each degree Celsius increase above the optimum temperature ${ }^{4}$, mortality increased by $2.72 \%$ for total mortality, $4.38 \%$ for mortality in those $\geq 65$ years of age, $1.86 \%$ for cardiovascular disease, and $12.82 \%$ for respiratory diseases. For temperatures below the optimum, mortality in the same categories declined by $1.37 \%, 2.05 \%, 1.69 \%$ and $5.15 \%$ for each degree Celsius increase.

It is important to note that people will probably acclimatise and adapt to the changing climate in time. Geographical variations of the temperature-mortality curve have been documented in studies from the United States, Europe and other countries, and results suggest that populations living in warmer areas have acclimatised to the warmer climate and higher summertime temperatures (Braga et al., 2002; Curriero et al., 2002; Keatinge et al., 2000). According to, for example, McMichael et al. (2004), global warming could result in an increase in the optimum temperature value as populations adjust to a warmer climate. The change in this optimum level for each scenario should reflect the rate of warming experienced, and is, therefore, assumed to be proportional to the projected change in average temperature. However, complete acclimatisation may take several years (McMichael et al., 1996). Dessai (2003) accounts for this

\footnotetext{
${ }^{4}$ Optimum temperature value is $16.5^{\circ} \mathrm{C}$ for total mortality, cardiovascular mortality, respiratory mortality, and mortality among those $\geq 65$ year of age.
} 
delay in acclimatisation by limiting the shift in optimum temperature levels to maximum $1^{\circ} \mathrm{C}$ per three decades.

Our calculations account for acclimatisation according to the following assumptions (see also Table 6.11):

1. No acclimatisation: the optimum temperature level remains the same.

2. Complete $(100 \%)$ acclimatisation, proportional to change in annual average temperature: The new optimum temperature level is calculated by adding the change in annual average temperature to the old optimum (i.e., the optimum temperature level in the baseline period).

3. Complete (100\%) acclimatisation, proportional to change in annual average temperature, but maximum $1^{\circ} \mathrm{C}$ per three decades: The new optimum temperature level is calculate by adding the change in annual average temperature to the old optimum, however this shift is limited to $1^{\circ} \mathrm{C}$ per three decades (maximum $2^{\circ} \mathrm{C}$ in 2050). The optimum temperature for these calculations only differ from the ones according to first acclimation assumption for the $\mathrm{W}+$ scenario, because in this scenario the annual average temperature increases faster than $1^{\circ} \mathrm{C}$ per three decades

4. Incomplete $(50 \%)$ adaptation, proportional to change in annual average temperature: the new optimum temperature level is calculated by adding half of the change in annual average temperature to the old optimum.

5. Complete $(100 \%)$ acclimatisation, proportional to change in summer average temperature. For the $\mathrm{G}+$ and $\mathrm{W}+$ scenarios, the increase in average summer temperature is higher than the increase in annual average temperature. Therefore, the new optimum temperature value for these two scenarios is also calculated using a second approach, which assumes that the change in optimum temperature is proportional to the change in average summer temperature.

Populations have also been shown to modify their sensitivity to heat and cold over time. However, as in the studies by McMichael et al. (2004) and Dessai (2003), we make no further assumption regarding changes in the mortality effect of temperatures above and below this optimum temperature level (no explicit adjustment for the effects of socio-economic or technological development on the exposure-effect relationship). In other words, we assume that populations remain equally vulnerable to departures from the optimum temperature value. 
Table 6.11: Optimum temperature level in each climate change scenario under different acclimatisation assumptions (for total mortality, cardiovascular mortality, respiratory mortality, and mortality among those aged 65 and over).

$\begin{array}{lll}\text { Acclimatisation assumption* } & \text { Scenario } & \text { Optimum } \\ \text { 1. No acclimatisation } & \text { All } & 16.5 \\ \text { 2. Complete (100\%) acclimatisation- proportional to change in } & \mathrm{G} & 17.4 \\ \text { annual average temperature } & \mathrm{G}+ & 17.8 \\ & \mathrm{~W} & 18.3 \\ & \mathrm{~W}+ & 19.1 \\ \text { 3. Complete (100\%) acclimatisation- proportional to change in } & \mathrm{G} & 17.4^{*} \\ \text { annual average temperature- limited to }+2^{\circ} \mathrm{C} \text { increase in } 2050 & \mathrm{G}+ & 17.8^{*} \\ & \mathrm{~W} & 18.3^{*} \\ & \mathrm{~W}+ & 18.5 \\ \text { 4. Incomplete (50\%) acclimatisation-- proportional to change } & \mathrm{G} & 17.0 \\ \text { in annual average temperature } & \mathrm{G}+ & 17.1 \\ & \mathrm{~W} & 17.4 \\ & \mathrm{~W}+ & 17.8 \\ \text { 5. Complete (100\%) acclimatisation- proportional to change in } & \mathrm{G}+ & 17.9 \\ \text { summer average temperature } & \mathrm{W}+ & 19.3 \\ \text { * Optimum is the same as with complete acclimatisation without limitation. }\end{array}$

Estimating temperature-related mortality attributable to climate change

The estimation of the change in heat-, cold- and temperature-related mortality is calculated using the $\mathrm{KNMI}^{\prime} 06$ scenarios for 2050, exposure-response relationships and acclimatisation assumptions described above, according to the following steps (based on McMichael et al. (2004)):

1) The daily temperature difference above (heat) or below (cold) the optimum temperature level is calculated for the baseline time series, as well as the transformed time series for each KNMI'06 scenario. For example, if the average temperature is above the optimum, people are exposed to heat. The level of exposure is measured as the difference between the average temperature and the optimum temperature. This temperature difference is calculated for both heat and cold for each day in the 30-year baseline and scenario periods. Table 6.11 provides an overview of the optimum temperature levels used.

2) These results are combined with the exposure-response relationships for heat and cold in order to calculate the annual proportion of temperatureattributable mortality (i.e., heat-attributable deaths plus cold-attributable deaths) for the baseline period as well as for each scenario.

3) The proportion temperature-related mortality attributable to climate change is calculated as the change in the annual proportion of temperature-related mortality for each climate scenario compared to the baseline climate. 


\subsubsection{Temperature-related mortality attributable to climate change}

For each climate scenario, the change in proportion of temperature-attributable deaths compared to the baseline is the result of the change in the proportion heat-attributable deaths and the change in the proportion cold-related deaths. The changes in these proportions cold-and heat-attributable deaths are, in turn, the result of:

- the change in temperatures below and above the optimum level (i.e., the change in exposure to cold, respectively heat)

- the change in mortality associated with a $1^{\circ} \mathrm{C}$ change below respectively above the optimum temperature.

The change in proportion of temperature-related deaths due to climate change is calculated for total mortality, cardiovascular mortality, respiratory mortality, and mortality among the elderly (65+), and under various acclimatisation assumptions. All results are given in the Appendix.

\section{Changes in temperature-related mortality without acclimatisation}

The change in exposure to heat respectively cold is calculated as the difference between a) the average exposure to heat respectively cold around 1990 and b) average exposure to heat respectively cold under the climate change scenario (2050). If climate change is not accompanied by acclimatisation, the optimum temperature remains unchanged at its 1990 level. Heat exposure increases with $0.17^{\circ} \mathrm{C}$ in $\mathrm{G}, 0.31^{\circ} \mathrm{C}$ in $\mathrm{G}+, 0.38^{\circ} \mathrm{C}$ in $\mathrm{W}$, and $0.73^{\circ} \mathrm{C}$ in $\mathrm{W}+$, while cold exposure decreases with $0.75^{\circ} \mathrm{C}$ in $\mathrm{G}, 1.00^{\circ} \mathrm{C}$ in $\mathrm{G}+1.45^{\circ} \mathrm{C}$ in $\mathrm{W}$, and $1.90^{\circ} \mathrm{C}$ in $\mathrm{W}+$. Although the rise in average temperature is distributed evenly throughout the year for the $G$ and $W$ scenarios (no change in air circulation), the decrease in cold exposure is larger than the increase in heat exposure. This is due to the fact that there are more cold days in the Netherlands than heat days. Both the changes in heat and cold exposure are higher in $G+$ and $W+$ compared to $G$ respectively $W$, because the altering air circulation patterns result in hotter summers as well as milder winters. However, the increase in summer temperatures is larger than the increase in winter temperatures. Hence, the increase in heat exposure in $\mathrm{G}+$ and $W+$ is about 1.9 times higher compared to $G$ respectively $W$, while the increase in cold exposure is only 1.3 times higher. 
Climate change, temperature and mortality in The Netherlands | 181
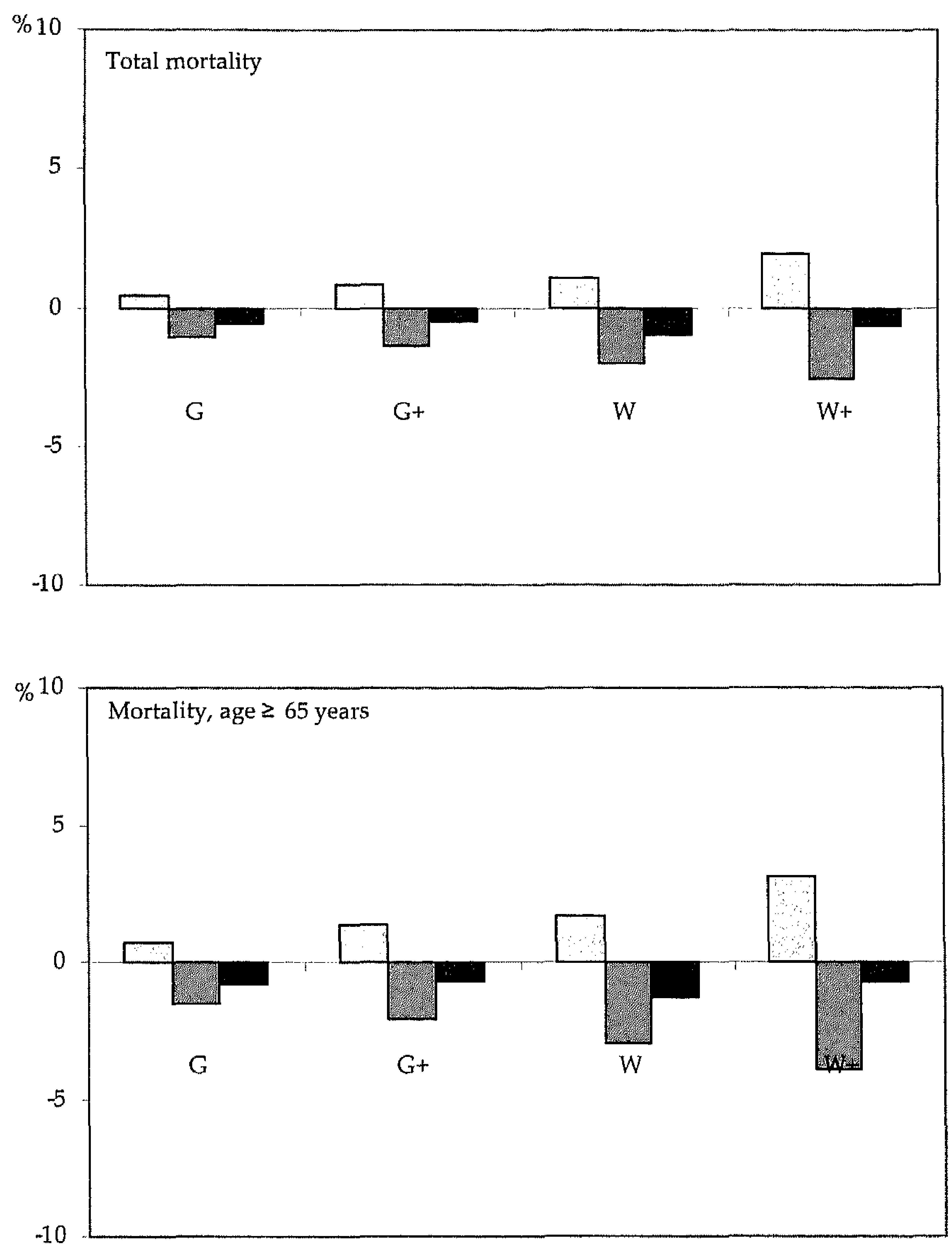

Figure 6.5: Change in temperature-related total mortality and mortality among those aged $\geq 65$ year, attributable to climate change in the KNMI'06 scenarios, without acclimatisation (2050 compared to 1990). 
182

Chapter 6
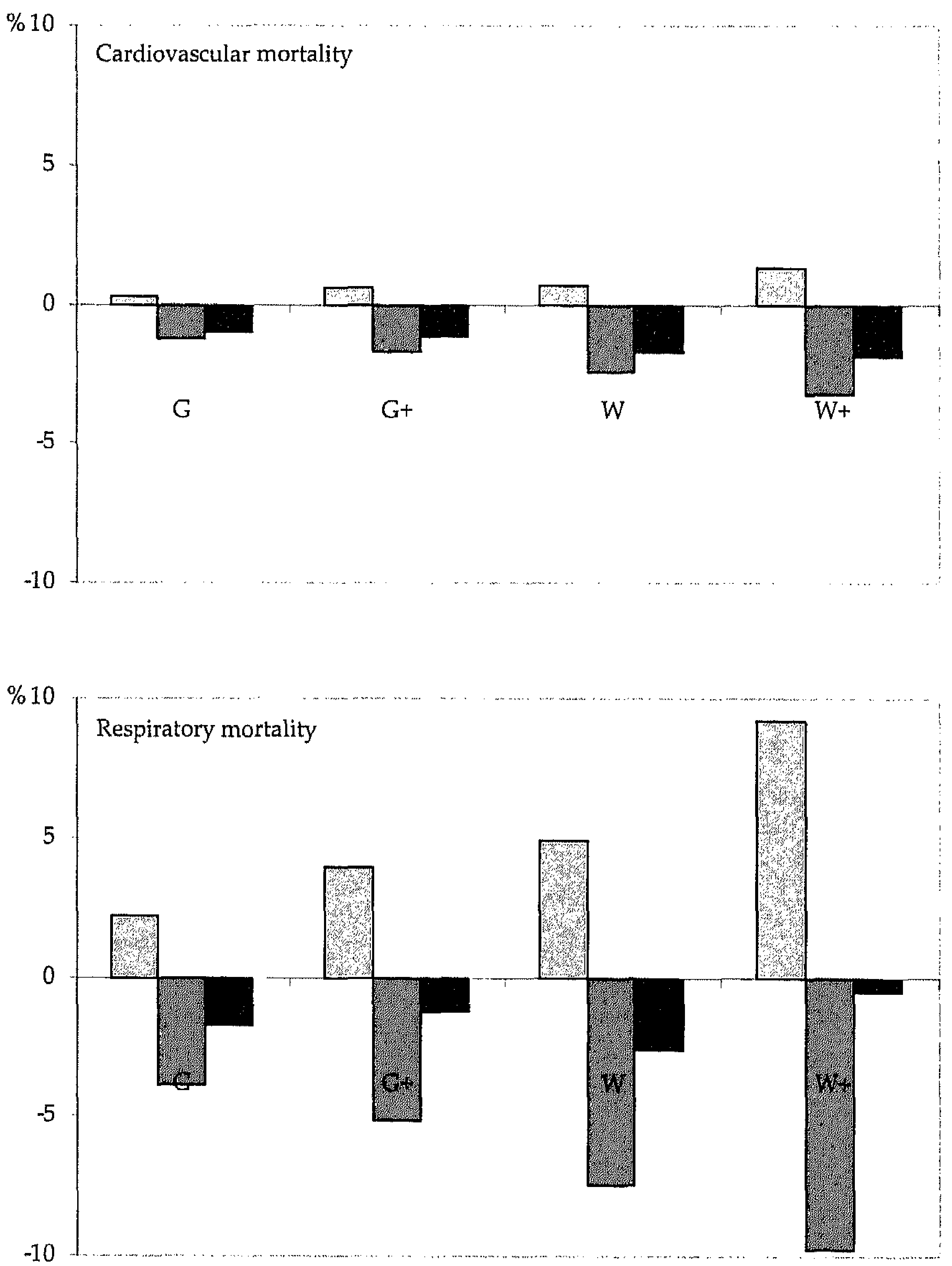

Figure 6.6: Change in temperature-related cardiovascular and respiratory mortality, attributable to climate change in the KNMI'06 scenarios, without acclimatisation (2050 compared to 1990). 
Figures 6.5 and 6.6 show the resulting change in temperature-related mortality attributable to climate change without acclimatisation. The proportion heatrelated mortality grows as the exposure to heat increases, but this is outweighed by the decrease in the proportion cold-related mortality. However, except for cardiovascular causes, the decrease in temperature-related mortality is less in case of changing air-circulation patterns. This can be explained by the interplay of two factors. First, as already mentioned above, the increase in summer temperatures for these scenarios $(\mathrm{G}+$ and $\mathrm{W}+)$ is higher than the increase in winter temperatures. Second, the change in mortality associated with $1^{\circ} \mathrm{C}$ increase is higher for heat than for cold (this difference is lowest for cardiovascular mortality).

Depending on the climate change scenario, the resulting relative decrease in the proportion temperature-related mortality is about $0.5-1.0 \%$ for total mortality, $1.0-1.9 \%$ for cardiovascular diseases, $0.5-2.6 \%$ for respiratory diseases and $0.7-$ $1.3 \%$ for mortality among the elderly (Appendix).

In 2100, the proportion heat-related mortality in each scenario further increases, while the proportion cold-related mortality further declines (results not shown); according to the $\mathrm{W}+$ scenario, this increase heat-related mortality outweighs the decrease in cold-related mortality resulting in an increase in the proportion temperature-related mortality (this is particularly the case for respiratory mortality, but not for cardiovascular mortality).

Changes in temperature-related mortality with acclimatisation

Under the complete acclimatisation assumption, the new optimum temperature level for each scenario is calculated by adding the increase in annual average temperature to the old optimum temperature. In other words, people adjust to the warmer climate, including both warmer summers and warmer winters. As a result, heat exposure and cold exposure do not differ much from the exposure levels in the baseline period for the G and W scenario (results not shown); there is no change in air circulation patterns and the increase in temperature is distributed rather evenly throughout the year.

For the G+ and $\mathrm{W}+$ scenarios, however, the increase in annual average temperature is not distributed evenly throughout the year. Hence, the increase in summer temperatures is higher than the increase in annual average temperature, and, subsequently, higher than the increase in optimum temperature level. As a result, the exposure to heat increases compared to the baseline. The increase in winter temperatures, in turn, is lower than the increase in annual average temperature, and, subsequently, lower than the increase in optimum temperature level. As a result, the exposure to cold compared to the baseline rises as well. Therefore, both heat- and cold-related mortality in these two scenarios increase for all selected causes of death (Figure 6.7). 

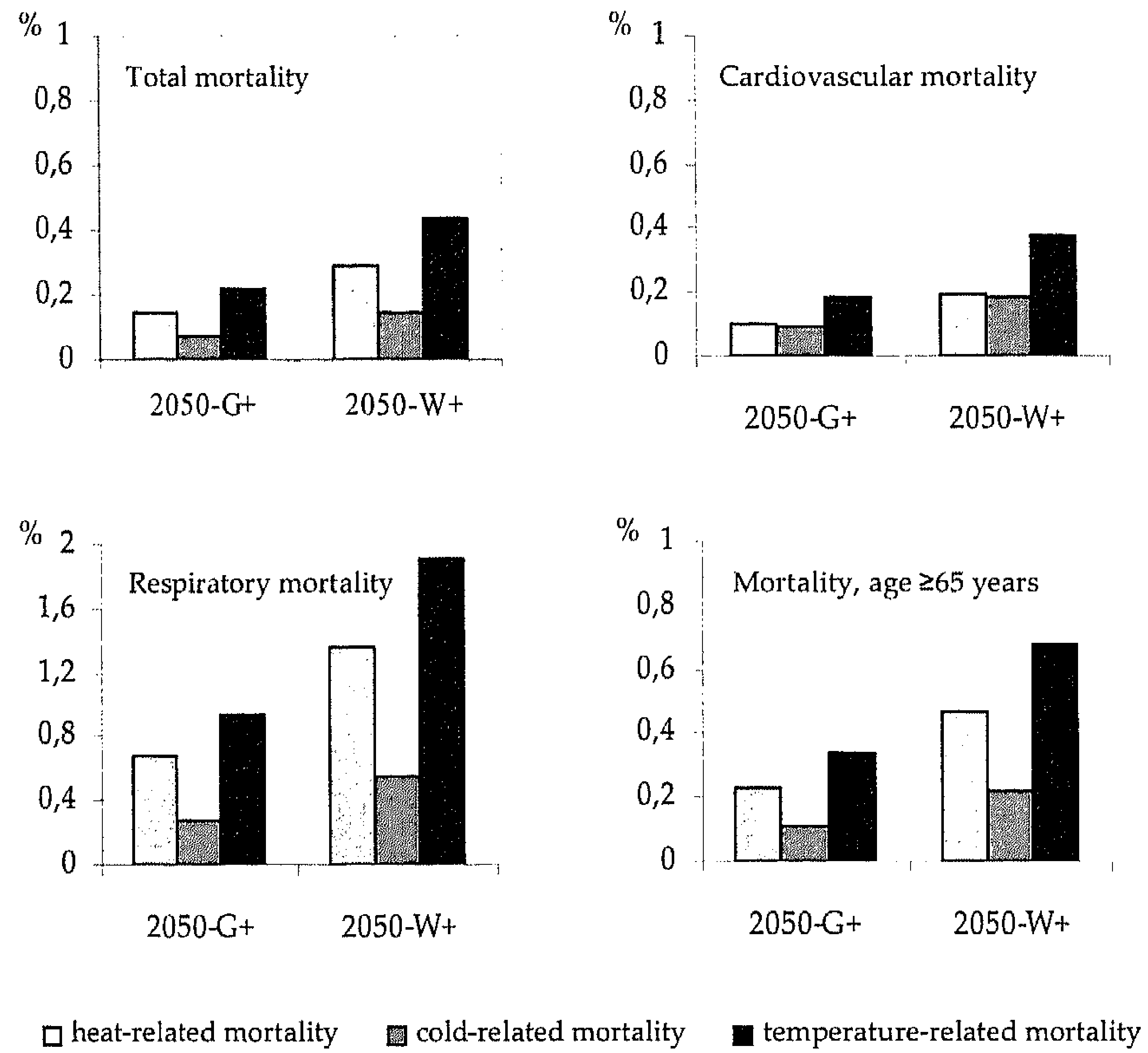

Figure 6.7: Change in temperature-related mortality attributable to climate change in the KNMI'06 G+ and W+ scenarios, with acclimatisation proportional to the change in annual average temperature (2050 compared to 1990).

When the acclimatisation is limited to $1^{\circ} \mathrm{C}$ per three decades, this only affects the change in optimum temperature level in the $\mathrm{W}+$ scenario, which is restricted to $2^{\circ} \mathrm{C}$ in 2050 . Compared to acclimatisation without restriction, this increases the heat-exposure and heat-related-mortality, while reducing cold-exposure and cold-related mortality. The net effect of the restricted acclimatisation is a lower increase in climate change attributable temperature-related mortality. For cardiovascular mortality, the acclimatisation restriction even results in a decrease in net temperature-related mortality compared to the baseline (Appendix). The results for the temperature-related mortality attributable to climate change under the 50\%-acclimatisation assumption are given in the Appendix. As expected, they fall in the range between the results for complete acclimatisation and no acclimatisation.

For the $G+$ and $W+$ scenarios, the change in optimum temperature level is also calculated proportional to the change in summer average temperature. Both heat- and cold-related mortality still increase in all scenarios compared to the baseline 1990. However, the increase in heat-related mortality is lower 
compared to the results for acclimatisation proportional to the change in annual average temperature, while the increase in cold-related mortality is higher; the assumption that acclimatisation will be proportional to the change in summer temperatures results in a higher increase in net temperature-related mortality attributable to climate change (Appendix).

\subsubsection{Discussion and conclusion}

Although global climate change is likely to be accompanied by an increase in summer temperatures, winters will likely be milder as well. The recent IPCC fourth assessment report (IPCC, 2007a) concludes that it is 'virtually certain' that climate change will result in reduced human mortality from decreased cold exposure, but this will 'very likely' be accompanied by an increased risk of heat-related mortality, particularly in vulnerable populations. Our results from the calculations without acclimatisation show that the decrease in cold-related mortality will be larger than the increase in heat-related mortality, but this difference is smaller in case of a change in air circulation patterns in Western Europe.

In case of acclimatisation proportional to the increase in annual average temperature, exposures to heat and cold in the $G$ and $W$ scenarios do not differ much from the exposure levels in the baseline period. In other words, people not only adjust to warmer summers, but also to warmer winters. For the $\mathrm{G}+$ and $\mathrm{W}+$ scenarios, however, the increase in annual average temperature is not distributed evenly throughout the year; our calculations accounting for acclimatisation (proportional to the change in annual average temperature) show that both the exposure to heat and cold increase, resulting in an increase in heat- and cold-related mortality compared to the baseline. For the G+ and $\mathrm{W}+$ scenarios, calculations were also performed assuming that the shift in the optimum temperature level will be proportional to the increase in summer average temperature, showing that the net temperature related-mortality rises even more; the increase in heat-related mortality is indeed smaller, but this is outweighed by the higher increase in cold-related mortality. Hence, in the scenarios without a change in air circulation patterns ( $G$ and $W$ ), temperaturerelated mortality declines or remains at about the baseline level (without respectively with acclimatisation). However, the change in air circulation patterns projected in the $\mathrm{G}+$ and $\mathrm{W}+$ scenarios yield different outcomes; temperature related mortality still decreases without acclimatisation but increases with acclimatisation.

Our calculations did not account for changes in the sensitivity of people to temperatures above and below the temperature level. Studies conducted in North America have shown a general decline in summer mortality, which was explained by the increased use of domestic space cooling (Davis et al., 2003; 
Donaldson et al., 2003). It is, of course, possible that in the future people might become less vulnerable to exposure to heat and cold; adaptive responses (e.g., air-conditioning, improved housing, and improved air quality) and public health interventions (e.g., heat-health warning systems) might affect the exposure-response relationship between mortality and temperature. For example, adaptation to climate change is likely to benefit from experience gained in reaction to extreme climate events, by specifically implementing proactive climate change risk management adaptation plans (IPCC, 2007a). Martens (1998b) conducted a sensitivity analysis (by varying the percental mortality effects between zero and the central estimate plus twice the standard deviation) showing that adaptations influencing the sensitivity to heat or cold are likely to change the temperature-related mortality due to climate change. However, sound quantitative information on the exact effect of adequate (policy) measures is still lacking. For example, there are as yet no robust assessments of the effectiveness of heat health warning systems (Kovats \& Ebi, 2006).

With regard to policy guidelines on the health effects of climate change, Article 2 of the United Nations Framework Convention on Climate Change (UNFCC) states that 'the ultimate objective of this Convention and any related legal instruments that the Conference of the Parties may adopt is to achieve (...) the stabilisation of greenhouse gas concentrations in the atmosphere at a level that would prevent dangerous anthropogenic interference.' However, in the policy arena, there is still considerable confusion about what qualifies as 'dangerous', as assessing when climate change becomes dangerous for the world is a complex task. The public based approach towards accepted health risks is based on what is acceptable to the general public. Bennet (1999) identified several 'fright factors' that influence the public's concern about risks and the public's risk. With regard to climate change, health risks are probably less accepted due to the nature of the climate change problem: involuntary, unfamiliar, manmade, the cause of hidden and irreversible damage and surrounded by scientific uncertainty. This was confirmed in a Dutch participatory workshop in 2004, during which the stakeholders indicated that any increase in mortality due to climate change was perceived as unacceptable (Gupta \& van Asselt, 2004).

Given the fact that the annual total mortality rate was between 8 and 9 per 1,000 population during the baseline period (Statistics Netherlands, 2006a), an increase in total mortality rate of only $0.2 \%$ would cause a health effect of approximately 0.017 per 1,000 . In 1990 the Dutch population was about 15 million, but this will increase to more than 17 million in 2050 according to the United Nations (UN) population prospects (medium-variant projection) (UN, 
$2003 \mathrm{~b}$ ). Based on these numbers, an increase of more than $0.2 \% 5$ in temperaturerelated total mortality in 2050 could result in more than 289 additional temperature-related deaths per year due to climate change. It is important to note that these numbers do not account for anticipated changes in population structure. Many countries have an ageing population. For the Netherlands, the number of persons aged $\geq 65$ years is projected to increase from about $13 \%$ in 1990 to $25 \%$ in 2050 (UN medium variant projection) (UN, 2003b). This ageing of the population will increase the proportion of vulnerable people in the population and climate change might represent an additional burden on this older population.

To conclude, for many temperate or cold regions, including the Netherlands, it is often stated that the decrease in cold-related mortality could counterbalance the increasing rates of heat-related mortality, even resulting in a reduction in mortality. This was also concluded in an earlier study by Martens and Huynen (2001). Our results based on the recent KNMI'06 scenarios shine a somewhat different light on the possible changes in Dutch temperature-related mortality related to global warming: climate change might decrease or increase temperature-related mortality in the Netherlands, depending on the climate change scenario and possible acclimatisation. It is important to note that our results for the Netherlands contrast with the IPCC's (2007a) conclusion that climate change will virtually certain reduce cold-related mortality. Cold-related mortality certainly decreases in all scenarios without acclimatisation. Our outcomes considering acclimatisation, on the other hand, show that in the scenarios with and without changing air circulation patterns cold-related mortality remains virtually unchanged respectively increases. However, given the uncertainties of social change it is still very challenging to assess how the interplay with socio-economic developments (e.g., adaptation, demographic changes) will play out (Comrie, 2007).

\footnotetext{
${ }^{5}$ Which is the case in $\mathrm{G}+$ and $\mathrm{W}+$ with acclimatisation proportional to the change in annual or summer temperature.
} 


\section{APPENDIX TO CHAPTER 6}

Table 6.12: Change (\%) in temperature-related total mortality attributable to climate change (KNMI'06 scenarios; 2050).

\begin{tabular}{|c|c|c|c|c|}
\hline 2050 & G & $\mathrm{G}+$ & W & $\mathbf{W +}$ \\
\hline \multicolumn{5}{|c|}{ 1. No acclimatisation (optimum temperature level remains $16.5^{\circ} \mathrm{C}$ ) } \\
\hline Heat-related mortality & 0.458 & 0.837 & 1.032 & 1.960 \\
\hline Cold-related mortality & -1.029 & -1.371 & -1.985 & -2.603 \\
\hline Temperature-related mortality & -0.571 & -0.534 & -0.953 & -0.643 \\
\hline \multicolumn{5}{|c|}{ 2. $100 \%$ acclimatisation-proportional to change in annual average temperature } \\
\hline Heat-related mortality & 0.013 & 0.142 & 0.028 & 0.288 \\
\hline Cold-related mortality & 0.007 & 0.072 & 0.014 & 0.145 \\
\hline Temperature-related mortality & 0.020 & 0.214 & 0.042 & 0.433 \\
\hline
\end{tabular}

3. $100 \%$ acclimatisation - proportional to change in annual average temperature-max. $1^{\circ} \mathrm{C}$ per three decades

Heat-related mortality

Cold-related mortality

Temperature-related mortality

$\begin{array}{lllr}* & * & * & 0.596 \\ * & * & * & -0.550 \\ * & * & * & 0.046\end{array}$

4. $50 \%$ acclimatisation - proportional to change in annual average temperature

$\begin{array}{lllll}\text { Heat-related mortality } & 0.220 & 0.458 & 0.464 & 0.999 \\ \text { Cold-related mortality } & -0.519 & -0.666 & -1.019 & -1.292 \\ \text { Temperature-related mortality } & -0.299 & -0.208 & -0.554 & -0.292\end{array}$

Temperature-related mortality $\quad-0.299 \quad-0.208 \quad-0.554 \quad-0.292$

5. $100 \%$ acclimatisation-proportional to change in summer average temperature ( $\mathrm{G}+$ and $\mathrm{W}+$ )

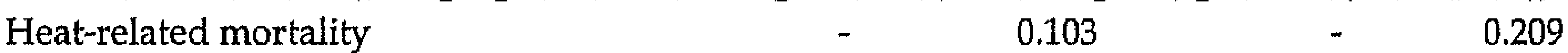

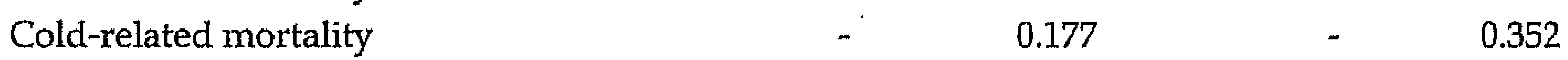

$\begin{array}{llll}\text { Temperature-related mortality } & - & 0.280 & -\end{array}$

* See: $100 \%$ acclimatisation- proportional to change in annual average temperature. 
Table 6.13: Change (\%) in temperature-related cardiovascular mortality attributable to climate change (KNMI'06 scenarios; 2050).

\begin{tabular}{|c|c|c|c|c|}
\hline 2050 & G & G+. & \multicolumn{2}{|c|}{$W_{+}$} \\
\hline \multicolumn{5}{|c|}{ 1. No acclimatisation (optimum temperature level remains $16.5^{\circ} \mathrm{C}$ ) } \\
\hline Heat-related mortality & 0.313 & 0.572 & 0.706 & 1.340 \\
\hline Cold-related mortality & -1.270 & -1.692 & -2.449 & -3.211 \\
\hline Temperature-related mortality & -0.956 & -1.119 & -1.743 & -1.870 \\
\hline \multicolumn{5}{|c|}{$2 \quad 100 \%$ acclimatisation-proportional to change in annual average temperature } \\
\hline Heat-related mortality & 0.009 & 0.097 & 0.019 & 0.009 \\
\hline Cold-related mortality & 0.008 & 0.088 & 0.018 & 0.008 \\
\hline Temperature-related mortality & 0.017 & 0.186 & 0.037 & 0.017 \\
\hline \multicolumn{5}{|c|}{$\begin{array}{l}3.100 \% \text { acclimatisation - proportional to change in annual average temperaturé max } 19 \mathrm{Cper} \\
\text { three decades }\end{array}$} \\
\hline Heat-related mortality & * & * & * & * \\
\hline Cold-related mortality & * & * & * & \\
\hline Temperature-related mortality & * & ${ }^{*}$ & * & * \\
\hline \multicolumn{5}{|c|}{$400 \%$ acdimatisation proportional to change in annual average temperature } \\
\hline Heat-related mortality & 0.150 & 0.313 & 0.318 & 0.683 \\
\hline Cold-related mortality & -0.641 & -0.821 & -1.257 & -1.593 \\
\hline Temperature-related mortality & -0.490 & -0.508 & -0.939 & -0.910 \\
\hline \multicolumn{5}{|c|}{ 5. $100 \%$ ad limatisation proportional to change in summer average temperature (G+ and WN) } \\
\hline Heat-related mortality & - & 0.070 & - & 0.143 \\
\hline Cold-related mortality & - & 0.218 & - & 0.434 \\
\hline Temperature-related mortality & - & 0.289 & - & 0.577 \\
\hline
\end{tabular}

* See: $100 \%$ acclimatisation- proportional to change in annual average temperature. 
Table 6.14: Change (\%) in temperature-related respiratory mortality attributable to climate change (KNMI'06 scenarios; 2050).

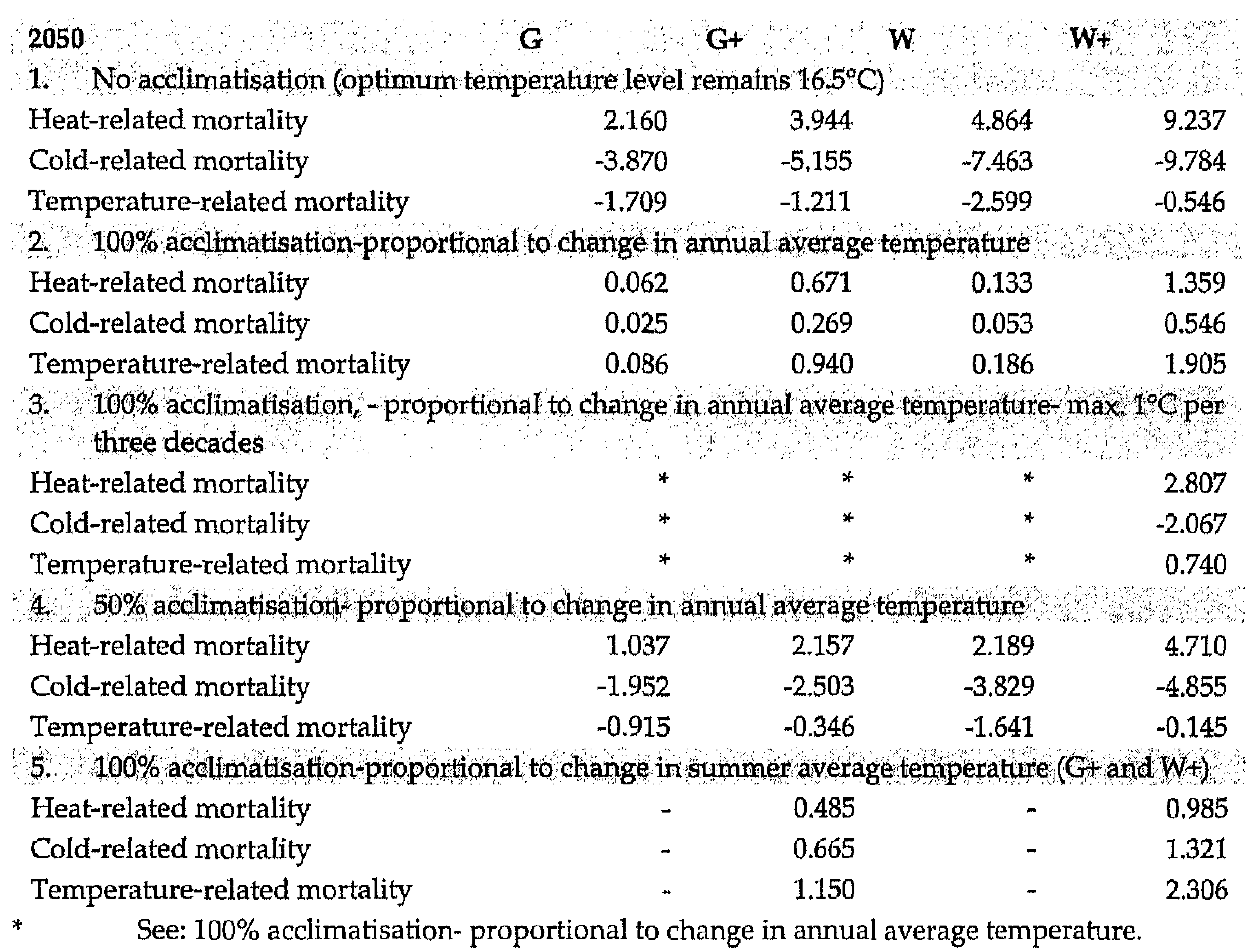


Table 6.15: Change (\%) in temperature-related mortality among the elderly (aged $\geq 65$ years) attributable to climate change (KNMI'06 scenarios; 2050).

G

G+

W

$W_{+}$

1. No acclimatisation (optimum temperature level remains $16.5^{\circ} \mathrm{C}$ )

Heat-related mortality

$0.738 \quad 1.348$

$-1.540 \quad-2.052$

1.662

3.156

Cold-related mortality

$-0.802$

$-0.704$

$-2.971$

$-3.894$

Temperature-related mortality

2 . $100 \%$ acclimatisation-proportional to change in annual average temperature

$-0.738$

Heat-related mortality

$\begin{array}{lll}0.021 & 0.229 & 0.045\end{array}$

0.464

Cold-related mortality

0.010

0.107

0.021

0.464

Temperature-related mortality

0.031

0.336

0.067

0.681

3. $100 \%$ acclimatisation - proportional to change in annual average temperature- $\max .1^{\circ} \mathrm{C}$ per three decades

Heat-related mortality

Cold-related mortality

Temperature-related mortality

* $\quad$ * $\quad * \quad * \quad 0.959$

* * * * $\quad * \quad-0.823$

* * * $\quad * \quad 136$

$4.50 \%$ acclimatisation proportional to change in annual average temperature

$\begin{array}{lllll}\text { Heat-related mortality } & 0.354 & 0.737 & 0.748 & 1.609\end{array}$

$\begin{array}{lllll}\text { Cold-related mortality } & -0.777 & -0.996 & -1.524 & -1.933\end{array}$

$\begin{array}{lllll}\text { Temperature-related mortality } & -0.423 & -0.259 & -0.777 & -0.324\end{array}$

5. $100 \%$ acclimatisation-proportional to change in summer average temperature ( $G+$ and $W+$ )

$\begin{array}{lllll}\text { Heat-related mortality } & - & 0.166 & - & 0.337\end{array}$

$\begin{array}{lllll}\text { Cold-related mortality } & - & 0.265 & - & 0.526\end{array}$

$\begin{array}{lllll}\text { Temperature-related mortality } & - & 0.430 & - & 0.863\end{array}$

* See: $100 \%$ acclimatisation- proportional to change in annual average temperature. 


\section{Chapter 7}

\section{LINKAGES BETWEEN BIODIVERSITY LOSS AND HEALTH}

Based on:

- Huynen, M.M.T.E., Martens, P., \& de Groot, R.S. (2004). Linkages between biodiversity loss and human health: a global indicator analysis. International Journal of Environmental Health Research, 14, 13-30. 


\section{LINKAGES BETWEEN BIODIVERSITY LOSS AND HEALTH}

Several global changes are believed to adversely affect human health, of which the loss in biodiversity is one. In the past century, social-economic developments have resulted in large improvements in human health and wellbeing, but these developments also have resulted in a significant reduction of biodiversity. Without major changes in human behaviour and policy, the human induced alterations in the (global) environment will continue to affect biodiversity (Chapin et al., 2000; Leemans, 1999; Sala et al., 2000; Secretariat of the Convention on Biological Diversity, 2006; WRI, 2000). The definitions of biodiversity show that it is the variability of the biotic part of the world's ecosystems and, therefore, it underpins the ability of the earth's ecosystems to provide most of it's goods and services (Rapport et al., 1998; Sala et al., 2000; Schulze \& Mooney, 1994; WRI, 2000). In the face of the changing biodiversity, we must consider the health consequences of such a change. Finding empirical proofs of the negative health consequences of biodiversity loss could be very important to stimulate political efforts regarding the conservation of the planet's biodiversity.

This Chapter addresses the possible relationship between loss of biodiversity and human health. Building on an earlier study by Sieswerda et al. (2001), we will explore the association between health and biodiversity loss by means of regression analysis, with control for socio-economic developments. For this quantitative analysis, we used indicators for human health, biodiversity and socio-economic development that were available at the country level.

\subsection{BIODIVERSITY, ECOSYSTEM FUNCTIONING AND HUMAN HEALTH}

In the early 1980 's, the notion of rapidly disappearing species resulted in the development of the concept of biodiversity. Accordingly, there has been an increasing interest and concern on the issue biodiversity in the past 25 years. Acknowledging the importance of biodiversity for human well-being, the Convention on Biological Diversity (CBD) (UNEP, 1992) aims to achieve by 2010 a significant reduction of the current rate of biodiversity loss at the global, regional and national level as a contribution to poverty alleviation and to the benefit of all life on Earth. But what does 'biodiversity' mean?

According to the $\mathrm{CBD}$, biodiversity is the variability among living organisms from all sources including, inter alia, terrestrial, marine and other aquatic ecosystems and ecological complexes of which they are part; this includes diversity within species, between species and ecosystems (UNEP, 1992). This is one of the most comprehensive definitions, but other (more simple) definitions of biodiversity are also used. For example, Leemans (1999) defines biodiversity as the collection of genes, species, communities and ecosystems, which 
constitute the living component of the earth's system. The UNEP $(1992,1995)$ distinguishes three sorts of biodiversity: genetic diversity, species diversity and ecological diversity. Genetic diversity refers to the variation in the genetic composition of individuals within or among species/populations. It can be measured in terms of, for example, variation between genes or between DNA or amino acid sequences, as well as the numbers of breeds, strains and distinct populations. Species diversity refers to the number and variety of species found in a given region. Ecological diversity refers to the numbers of species in given areas, the ecological roles that these species play, the way that the composition of species changes as we move across the regions, and the grouping of species (ecosystems) that occur in particular areas, together with the processes and interactions that take place within and between that systems. It also covers the diversity of ecosystems in landscapes, of landscapes in biomes and of biomes on the planet. These definitions show that biodiversity encompasses multiple components or levels. One must also notice that biodiversity always relates to the variability of genes, species and ecosystems within a defined area and time span; the concept 'biodiversity' is space and time related.

Today, the environment, including biodiversity, is exceedingly threatened by a combination of multiple human impacts. The extinction of species is a natural phenomenon, but human activities have increased the extinction rate by approximately a factor 1,000 to 10,000 of the natural rate (Pimm et al., 1995). Numerous animal and plant species have already disappeared due to human impacts. The extinction of the Dodo from the island Mauritius is one of the most famous examples. 'Loss of biodiversity' is often linked with the threatening of whales, tigers, rhino's, and orang-utans. But these more 'charismatic' species just form a very small part of the total biodiversity. Large numbers of (rather) unknown organism are threatened by extinction, or have already disappeared. However, one must also not forget that the loss of biodiversity does not only constitute of the extinction of species. Perhaps even more important are the negative impacts of human activities on the number of organisms and populations per species, on the species-distribution (van Soest, 1998). The literature mostly focuses on the loss of species diversity, but genetic diversity and ecosystem diversity are, of course, adversely affected by humans as well.

The driving forces behind the increasing scale of human pressures on biodiversity are population growth and social-economic developments. Human development has resulted in the massive conversion of natural areas. Loss of habitat area is considered as the most important direct pressure (WRI, 2000). The Millennium Ecosystem Assessment (MA) (Hassan et al., 2005) concluded that habitat conversion, degradation, and fragmentation on land (e.g., due to agricultural activities) and in the oceans (e.g., due to fishing) have been the most important direct drivers of biodiversity loss globally in the recent past. Although habitat change will continue to be an important threat to biodiversity, 
the impacts of climate change, invasive non-native species, pollution, and nutrient overload are all increasingly important.

Scenario analysis (Sala et al., 2000) showed that by the year 2100 land-use change is expected to have the largest global impact on biodiversity, due to negative effects on habitat availability. The second most important driving force is expected to be the estimated climate change, mostly because of the increase in temperatures at higher latitudes. Changes in atmospheric $\mathrm{CO}_{2}$, biotic exchange and nitrogen deposition will also play an important role in the declining biodiversity, although the relative importance is regionally variable.

In the face of the large changes in biodiversity, the question is if and how this affects human health. Grifo and Rosenthal (1997) published one of the first books on this subject entitled 'Biodiversity and Human Health'. Suggested consequences of biodiversity loss in this book are the spread of human diseases, loss of medical models, diminished supplies of raw materials for drug discovery and biotechnology, and threats on food production and water quality.

Maintaining a certain level of biodiversity is necessary for proper ecosystem functioning and the provisions of ecosystem services to mankind (Chapin et al., 2000; Hassan et al., 2005; Sala et al., 2000; Schulze \& Mooney, 1994; WRI, 2000). In other words, biodiversity underpins the ability of the earth's ecosystems to provide most if its goods and services. Ecosystems are communities of interacting organisms and the physical environment in which they live. They consist of organic and inorganic matter and natural forces that interact and exchange; they are living sums greater than their parts (WRI, 2000). Ecosystems provide us with most basic necessities (e.g., food, fibre, water) and essential services (e.g., air and water purification, climate control, nutrient recycling, soil production), which are the result of the natural processes within the ecosystem. These processes, in turn, result from the many and complex interactions between the living organisms (biota) and the chemical and physical (abiotic) components of the ecosystem (de Groot et al., 1999). De Groot (1992) defined ecosystem functions as the capacity of natural processes and components to provide goods and services that satisfy human needs (directly and/or indirectly). Human needs can be divided into physiological needs (need for oxygen, water, food, physical health and a healthy unpolluted environment) and psychological needs (need for mental well being). The degree of satisfaction of human needs (including good health) depends on certain environmental conditions, which can be translated in terms of functions of the natural environment. De Groot et al. (2002) identified and classified a comprehensive overview of ecosystem functions in four different groups: production, regulation, information and habitat functions. Table 7.1 gives an indication of our dependence on the natural systems and the biodiversity within. Therefore, compromising the ecosystem's ability to provide goods and services to humans can result in several socio-economic impacts. 
Table 7.1: Ecosystem functions, goods and services (de Groot et al., 2002).

\section{Ecosystem function}

Regulatory functions:

This group of functions relates to the capacity of natural and semi-natural ecosystems to regulate essential ecological processes and life support systems through biogeochemical cycles and other biospheric processes. In addition to maintaining ecosystem (and biosphere) health, these regulation functions provide many services, which have direct and indirect benefits to humans (such as clean air, water and soil, and biological control services).

Habitat functions:

Natural ecosystems provide refuge and reproductionhabitat to wild plants and animals and, thereby, contribute to the (in situ) conservation of biological and genetic diversity and evolutionary processes.

Production functions:

Photosynthesis and nutrient uptake by autotrophs converts energy, carbon dioxide, water, and nutrients into a wide variety of carbohydrate structures, which are then used by secondary producers to create an even larger variety of living biomass. This broad diversity in carbohydrate structures provides many ecosystem goods for human consumption, ranging from food and raw materials to energy resources and genetic material.

Information functions:

Because most of human evolution took place within the context of undomesticated habitat, natural ecosystems provide an essential 'reference function' and contribute to the maintenance of human health and well-being by providing opportunities for reflection, spiritual enrichment, cognitive development, re-creation and aesthetic experience.
Goods and services (examples)

- Gas regulation

- Climate regulation

- Disturbance prevention (e.g. flood prevention)

- Water regulation

- Water supply

- Soil retention

- Soil formation

- Nutrient regulation

- Waste treatment

- Pollination

- Biological control (e.g. control of pests and diseases)

- Refugium function (suitable living space)

Nursery function (suitable reproduction habitat)

- Food

- Raw materials (e.g. fuel, building materials)

- Genetic resources

- Medicinal resources

- Ornamental resources

- Aesthetic information (enjoyment of scenery)

- Recreation

- Cultural \& artistic information

- Spiritual and historic information

- Science \& education

Among these adverse effects of declining ecosystem functioning are the negative implications for human health (Corvalan et al., 2005; Rapport et al., 1998). Several authors have addressed the link between biodiversity and ecosystem functioning (Chapin et al., 2000; Folke et al., 1996; Mooney, 1996; Schulze \& Mooney, 1994; Schwartz et al., 2000; UNEP, 1995), but it is still unclear which ecosystem functions are primarily important to sustain our health. Basically, the following types of 'health functions' can be distinguished. 
First, ecosystems provide us with basic human needs like food, clean air, clean water and clean soils. Secondly, they prevent the spread of diseases through biological control. Third, ecosystems provide us with medical and genetic resources, which are necessary to prevent or cure diseases. Finally, biodiversity also contributes to the maintenance of mental health by providing opportunities for recreation and cognitive development. Thus, biodiversity loss could result in compromised ecosystem functions, which, in turn, could negatively influence our health.

\subsection{METHODS}

The selected indicators for human health, (loss of) biodiversity, and socioeconomic development are described in the next section. Accordingly, the performed quantitative analyses are discussed. The crude association between the selected indicators is studied by means of the Spearman correlation, while the corrected association between health and biodiversity indicators is studied by means of least squares multiple regression. For these statistical analyses, we made use of SPSS 10.0 .

\subsubsection{Selected indicators}

Our indicator database contained 252 countries, but not one of the selected indicators was available for all of these countries. The indicators were derived from the World Resources Report 1996-1997 (WRI, 1996) and the associated database CD-ROM, World Resources Report 2000-2001 (WRI, 2000) and the associated database CD-ROM, World Development Indicators Report 1999 (World Bank, 1999) and the associated database CD-ROM, and the World Health Report 2000 (WHO, 2000). Ideally, we would have selected data on biodiversity measured several years (for example ten years) earlier than the data used on human health in order to explore possible time lags between losses in biodiversity and the resulting health consequences. Unfortunately, this was not possible, because the indicators on biodiversity were not measured so many years prior to the health data. Therefore, we used the biodiversity indicators for the years that they were measured and the health indicators for the most recent years that they were available1. See Table 7.2 for an overview of the selected indicators.

\footnotetext{
1 This analysis was performed in 2001-2002. Since then, a provisional list of global indicators has been formulated under the CBD in order to assess trends in biodiversity; these indicators are now being further developed and tested (CBD, 2007).
} 
Indicators of human health

Because it is still unknown which health indicators of endpoints are most vulnerable to reductions in biodiversity, we selected three health indicators reflecting different aspects of our health (which are also selected by the World Health Organization (WHO) in their list of basic health indicators): life expectancy at birth in years (5-year average over the period 1995-2000), infant mortality rate per 1,000 live births (5-year average over the period 1995-2000) and incidence of low-birthweight babies (percentage of births, for most recent year in the range 1993-1996). Besides these traditional measures of health, we also selected the Disability Adjusted Life Expectancy (DALE) calculated at birth (WHO, 2000). This is a relatively new measure, which, unlike the ordinary life expectancy, also accounts for the years lived in ill health. This indicator was only available for 1999.

\section{Indicators of biodiversity loss}

Unlike for human health and socio-economic development, no broadly accepted core-set of indicators for biodiversity exists (Soberon et al., 2000). There are some indicators available, but these are collected for other purposes than investigating the relationship between biodiversity and human health. We selected three biodiversity indicators 2 based on face validity: the proportion threatened species as percentage of known species, current forest as percentage of original forest and percentage of land highly disturbed by human activities. The threat to species is one of the most studied and discussed aspects of biodiversity loss and for this reason we selected the proportion threatened species as percentage of known species. The number of threatened species includes full species that are categorised by the World Conservation Union (IUCN) as being critically endangered, endangered, or vulnerable (WRI, 2000). We first calculated this proportion for mammals, birds, reptiles, amphibians, and higher plants separately. Accordingly, we averaged these different percentages into one mean indicator. The data we used referred to the 1990s (WRI, 2000). Forests harbour about two-thirds of the known terrestrial species and have the highest species diversity and endemism (species native to a particular region or ecosystem and occurring nowhere else) of any ecosystem. Therefore, we selected current forest as percentage of original forest as an indicator (WRI, 2000). Current forest refers to estimated closed forest cover. Original forest as a percent of land area refers to the estimate of the percent of land that would have been covered by closed forest about 8,000 years ago assuming current climatic conditions, before large-scale disturbance by human society began. We used data for 1996.

\footnotetext{
2 We did not include indicators of intermediate factors (e.g., the availability of safe drinking water) in our regression model; these factors might be in the causal pathway, so controlling for them in the model might adjust away part of the effect under study (Sieswerda et al., 2001).
} 
We also wanted to include an indicator of biodiversity at the ecosystem level. However, very few studies focussed on biodiversity at this level (Izsak \& Papp, 2000; Leemans, 1999) and no suitable indicator of the state of ecosystem diversity was available. Therefore, we chose to include an indicator that reflected the pressure on the ecosystems: percentage of land highly disturbed by human activities (WRI, 1996). The World Resources Institute (WRI) defined high human disturbance as follows: under permanent agricultural cultivation or urban settlement, and/or contain primary vegetation removed without evidence of re-growth; contain current vegetation differing from potential vegetation; have a record of desertification or other permanent degradation (WRI, 1996). This indicator was only available for 1993.

Please note that the percentage threatened species was log-transformed in order to meet the linearity assumption in the regression analysis.

\section{Indicators of socio-economic development}

The threat to biodiversity, and the resulting effects on human health, cannot be seen apart from societal processes, like social-cultural and economic developments. The effects on human health could be masked or distorted by the effect of confounding (Briggs et al., 1996). In this study, we consider a confounder to be a variable that is correlated with biodiversity, while it also is a determinant of health. It is very probable that social-cultural and economic developments can act as confounding factors of the relationship between biodiversity and human health. The observed crude (i.e., without control for confounding) association between loss of biodiversity and our health could, therefore, be different than with control for confounding.

We selected three key measures of economic and social development, namely Gross National Product (GNP) per capita in current US\$ calculated using the Atlas Method (3-year average over the period 1995-1997), development grade (for 2000) and adult illiteracy rate (for 1997). Beside these three general socioeconomic indicators, we also wanted to include indicators for the development of the health-care system, being directly related to health. The selected indicators for the development of the health care system are: health expenditure as percentage of GDP (for 1997) and percentage of one year olds immunised (averaged for DTP, measles, polio and tuberculosis; data for most recent year within the range 1995-1997).

Please note that GNP per capita was log-transformed in order to meet the linearity assumption in the regression analysis. 
Table 7.2: Overview of selected indicators.

\begin{tabular}{|c|c|c|c|}
\hline $\begin{array}{l}\text { Indicator } \\
\text { Biodiversity }\end{array}$ & Source & Year(s) & Notes \\
\hline $\begin{array}{l}\text { Percentage } \\
\text { threatened species }\end{array}$ & $\begin{array}{l}\text { The World } \\
\text { Resources CD-ROM } \\
2000-2001^{*}\end{array}$ & $1990 \mathrm{~s}$ & $\begin{array}{l}\text { Mean of mammals, birds, } \\
\text { reptiles, amphibians, higher } \\
\text { plants }\end{array}$ \\
\hline $\begin{array}{l}\text { Percentage of land } \\
\text { highly disturbance } \\
\text { by human activities }\end{array}$ & $\begin{array}{l}\text { The World } \\
\text { Resources CD- ROM } \\
\text { 1996-1997* }\end{array}$ & 1993 & \\
\hline $\begin{array}{l}\text { Current forest as } \\
\text { percentage of } \\
\text { original forest }\end{array}$ & $\begin{array}{l}\text { The World } \\
\text { Resources CD-ROM } \\
2000-2001^{*}\end{array}$ & 1996 & \\
\hline \multicolumn{4}{|l|}{ Health } \\
\hline $\begin{array}{l}\text { Life expectancy at } \\
\text { birth }\end{array}$ & $\begin{array}{l}\text { The World } \\
\text { Resources CD-ROM } \\
2000-2001^{*}\end{array}$ & $1995-2000$ & 5-year average \\
\hline $\begin{array}{l}\text { DALE (Disability } \\
\text { Adjusted Life } \\
\text { Expectancy) }\end{array}$ & $\begin{array}{l}\text { World Health Report } \\
2000^{* *}\end{array}$ & 1999 & \\
\hline $\begin{array}{l}\text { Infant mortality rate } \\
\text { (per } 1,000 \text { live births) }\end{array}$ & $\begin{array}{l}\text { The World } \\
\text { Resources CD-ROM } \\
\text { 2000-2001* }\end{array}$ & $1995-2000$ & 5-year average \\
\hline $\begin{array}{l}\text { Percentage low- } \\
\text { birthweight babies }\end{array}$ & $\begin{array}{l}\text { World Development } \\
\text { Indicators CD- ROM } \\
1999^{* * *}\end{array}$ & $1993-1996$ & $\begin{array}{l}\text { Data are for the most recent } \\
\text { year within the range given }\end{array}$ \\
\hline \multicolumn{4}{|c|}{ Socioeconomic confounders } \\
\hline $\begin{array}{l}\text { GNP per capita, } \\
\text { (Atlas method; } \\
\text { current US\$) }\end{array}$ & $\begin{array}{l}\text { World Development } \\
\text { Indicators CD- ROM } \\
1999^{* * *}\end{array}$ & 1995-1997 & 3-year average \\
\hline Development grade & $\begin{array}{l}\text { World resources } 200- \\
2001^{*}\end{array}$ & 2000 & $\begin{array}{l}1=\text { least developed countries, } \\
2=\text { developing countries } \\
3=\text { industrialised countries }\end{array}$ \\
\hline $\begin{array}{l}\text { Adult illiteracy rate } \\
\text { (\% of people aged } 15 \\
\text { and above) }\end{array}$ & $\begin{array}{l}\text { World Development } \\
\text { Indicators CD- ROM } \\
1999^{* * *}\end{array}$ & 1997 & \\
\hline $\begin{array}{l}\text { Total health } \\
\text { expenditure as } \\
\text { percentage of GDP }\end{array}$ & $\begin{array}{l}\text { World Health Report } \\
2000^{* *}\end{array}$ & 1997 & \\
\hline $\begin{array}{l}\text { Percentage one year } \\
\text { olds immunised }\end{array}$ & $\begin{array}{l}\text { The World } \\
\text { Resources CD-ROM } \\
\text { 2000-2001* }\end{array}$ & $1995-1997$ & $\begin{array}{l}\text { Mean of immunisation } \\
\text { against measles, polio, } \\
\text { tuberculosis and DTP (data } \\
\text { are for the most recent year } \\
\text { within the range given) }\end{array}$ \\
\hline $\begin{array}{l}\text { World Resoul } \\
\text { World Health } \\
\text { The World Ba }\end{array}$ & $\begin{array}{l}\text { Institute } \\
\text { ganization }\end{array}$ & & \\
\hline
\end{tabular}




\subsubsection{Spearman correlation and regression analysis}

We used Spearman correlations and scatterplots to indicate the direction and strength of the crude associations between the different indicators. Ordinary least squares multiple regression (Allison, 1999; Fox, 1997; Krzanowski, 1998; Ramsey \& Schafer, 1997; van Breukelen, 1995) was used to determine the relationship between the health- and biodiversity-indicators with control for socio-economic confounding factors. The general formula for a multiple regression model is

$$
Y=\beta_{0}+\beta_{1}{ }^{*} X_{1}+\beta_{2}{ }^{*} X_{2}+(\ldots)+e,
$$

where $Y$ is called the dependent variable (in this study: health indicator) and the X's the independent variables (in this study: biodiversity and socioeconomic indicators). The $B^{\prime} s$ are the regression coefficients, which are determined through the regression modelling process. When a coefficient of a particular independent variable is zero, the corresponding $X$ does not contribute to the prediction of $Y$; it is concluded that this independent variable is no good predictor of the dependent variable. The $e$ in the equation stands for the error of prediction, also called the residual: the distance between the predicted value of $Y$ and the observed $Y$.

Several indicators showed a skewed distribution. The non-normality of these indicators caused, however, no problems in our analysis, because we used the non-parametric Spearman correlation coefficient to calculate the correlations between the indicators and, secondly, because normality of the independent and dependent variables is not a requirement for multiple least squares linear regression. In order to obtain unbiased, effective estimates of the regression coefficients that can be tested for significance using the t-test, we first checked whether the models met the assumptions regarding linearity, mean independence, constant variance (also called homoscedascity) and normality of the errors of prediction (Allison, 1999; Fox, 1997; Ramsey \& Schafer, 1997; van Breukelen, 1995).

We considered a case as an outlier respectively an influential case, when the value of its studentised residual is not in the range from -3 to 3 , or when its Cook's distance is higher then 1.0 and when these values are also very distant from the studentised residuals or Cook's distances of the other cases (van Breukelen, 1995). These outliers and influential cases were deleted from the regression analysis (Allison, 1999; Fox, 1997; Ramsey \& Schafer, 1997; van Breukelen, 1995). Multicolinearity was detected by means of Variance Inflation Factors (VIF); in this study, a VIF above 2.5 is considered as a indication of multicolinearity (Allison, 1999). When multicolinearity is observed between two conceptually related variables, one of them is deleted from the model. 
The $t$-statistic tests the hypothesis that each regression coefficient is equal to zero, i.e., the independent variable (biodiversity or socio-economic indicator) corresponding to the coefficient is not a good predictor of the outcome variable (health indicator) (Allison, 1999; Fox, 1997; Krzanowski, 1998; Ramsey \& Schafer, 1997; van Breukelen, 1995). We reject this hypothesis if the significance level is below 0.1.3 The R-squared gives the percent of variation in the dependent variable that is explained by the independent variables. The adjusted R-square is a modification of the R-square that adjusts for the number of independent variables (Allison, 1999; van Breukelen, 1995). The larger the Rsquared, the better the fit of the regression line to the data (Krzanowski, 1998). The independent variables that have to be included in the final model of a particular dependent variable are determined by means of backward deletion, in order to obtain a parsimonious model (Allison, 1999; van Breukelen, 1995) (this procedure was not performed by means of an automatised procedure). We chose only to consider confounders for exclusion and not the biodiversity indicators (even when they have significance levels higher than 0.1), because the latter are the main focus of the study (Allison, 1999).

\subsection{RESULTS}

There was no correlation among the biodiversity indicators, while the socioeconomic indicators were all significantly correlated with each other. The four health indicators were also all significantly correlated with each other (results not shown). As expected, all socio-economic indicators were significantly correlated with all health indicators in such direction that an increase in socioeconomic development was positively associated with better health. All socioeconomic indicators were significantly related with at least one indicator of biodiversity and, therefore, they all should be considered as a possible confounder of the health-biodiversity-relationship. However, a closer look at the correlations between the biodiversity and socio-economic indicators showed no convincing pattern of an increasing loss of biodiversity with an increasing socio-economic development. First, the correlations are not extremely high (the highest correlation was the one between percentage of highly disturbed land and development grade and had a value of 0.263 ). Second, the direction of the significant correlations between the percentage remaining forest and socio-

\footnotetext{
${ }^{3}$ We use a higher threshold-value then most studies in order to increase the power of the tests. The power is the probability of rejecting a hypothesis when it is indeed false. Due to the fact that in our models the number of independent variables is rather large in comparison with the number of cases, there is a higher chance of not rejecting the hypothesis when it really should be rejected. Making the conditions for rejection less stringent (increasing the threshold of the p-value) decreases the change of making such a mistake (Krzanowski, 1998; van Breukelen, 1995).
} 
economic development was not consistent. And third, there was no correlation at all between (Log) threatened species and one of the socio-economic indicators.

\subsubsection{Crude associations between health and biodiversity}

To give an indication of the crude association between health and biodiversity, Table 7.3 shows the correlation between each health and biodiversity indicator In this section, only significant correlations are discussed. It is important to note that the crude associations could be biased due to confounding factors. The following significant crude associations between the health and biodiversity indicators were observed:

- Life expectancy had a significant positive correlation with the logarithm of the percentage threatened species $(r=0.203)$ and the percentage highly disturbed land $(r=0.268)$.

- DALE had a significant positive correlation with current forest as percentage of original forest $(\mathrm{r}=0.153)$ and with percentage highly disturbed land $(\mathrm{r}=0.278)$.

- Infant mortality rate had a significant negative correlation with the logarithm of the percentage threatened species $(r=-0.133)$, the percentage current forest of original forest $(r=-0.144)$ and the percentage highly disturbed land $(-0.279)$.

- The percentage low-birth-weight babies had a significant negative correlation with the percentage of land highly disturbed by humans ( $\mathrm{r}=-$ 0.185).

Table 7.3: Spearman correlation between the health and biodiversity indicators.

\begin{tabular}{|c|c|c|c|c|}
\hline Spearman correlations & Life & DALE & Infant & $\begin{array}{l}\% \text { ow birth } \\
\text { weight babies }\end{array}$ \\
\hline $\begin{array}{l}\text { (LOG) \% threatened } \\
\text { species }\end{array}$ & $0.203^{* *}$ & 0.116 & $-0.133^{*}$ & 0.100 \\
\hline $\begin{array}{l}\% \text { current forest of } \\
\text { original forest }\end{array}$ & 0.119 & $0.153^{*}$ & $-0.144^{*}$ & -0.054 \\
\hline$\%$ land highly disturbed & $0.268^{* *}$ & $0.278^{* *}$ & $-0.279^{* *}$ & $-0.185^{*}$ \\
\hline $\begin{array}{l}\text { health expenditure as \% } \\
\text { of GDP }\end{array}$ & $0.540^{* *}$ & $0.573^{* *}$ & $-0.571^{* *}$ & $-0.439^{* *}$ \\
\hline $\begin{array}{l}\% 1 \text {-year olds } \\
\text { immunised }\end{array}$ & $0.551^{* *}$ & $0.557^{* *}$ & $-0.555^{* *}$ & $-0.392^{* *}$ \\
\hline Illiteracy rate & $-0.663^{* * *}$ & $-0.688^{* *}$ & $0.742^{* *}$ & $0.582^{* *}$ \\
\hline (LOG) GNP per capita & $0.862^{* *}$ & $0.845^{* *}$ & $-0.891^{* *}$ & $-0.708^{* *}$ \\
\hline $\begin{array}{l}\text { development grade } \\
p<0.1 ; * * 0<0.01\end{array}$ & $0.796^{* *}$ & $0.807^{* *}$ & $-0.830^{* *}$ & $-0.637^{* *}$ \\
\hline
\end{tabular}




\subsubsection{Adjusted associations between health and biodiversity}

In the regression analysis, we explored the biodiversity-health relationship with control for the socio-economic confounders. In all these models, the percentage threatened species and GNP per capita were log-transformed in order to meet the linearity assumption and development grade was deleted to avoid multicolinearity with Log GNP per capita.

Adjusted association between life expectancy and biodiversity

Table 7.4 shows the regression results for life expectancy. The final model of life expectancy contained the following socio-economic variables: percentage oneyear-olds immunised, illiteracy rate and the logarithm of GNP per capita. Health expenditure as percentage of GNP was deleted, because it had a nonsignificant association with life expectancy in this model.

Of the biodiversity indicators, only the logarithm of the percentage threatened species had a regression coefficient that was significantly different from zero; an increase in the logarithm of the percentage threatened species was significantly associated with an increase in life expectancy. The percentage current forest of original forest showed no association with life expectancy, with or without control for socio-economic development. The crude association between percentage highly disturbed land and life expectancy disappeared after control for the socio-economic confounders.

Of the social-economic indicators in the final model, the logarithm of GNP per capita had the strongest association with life expectancy.

Table 7.4: Results of the final regression model of life expectancy

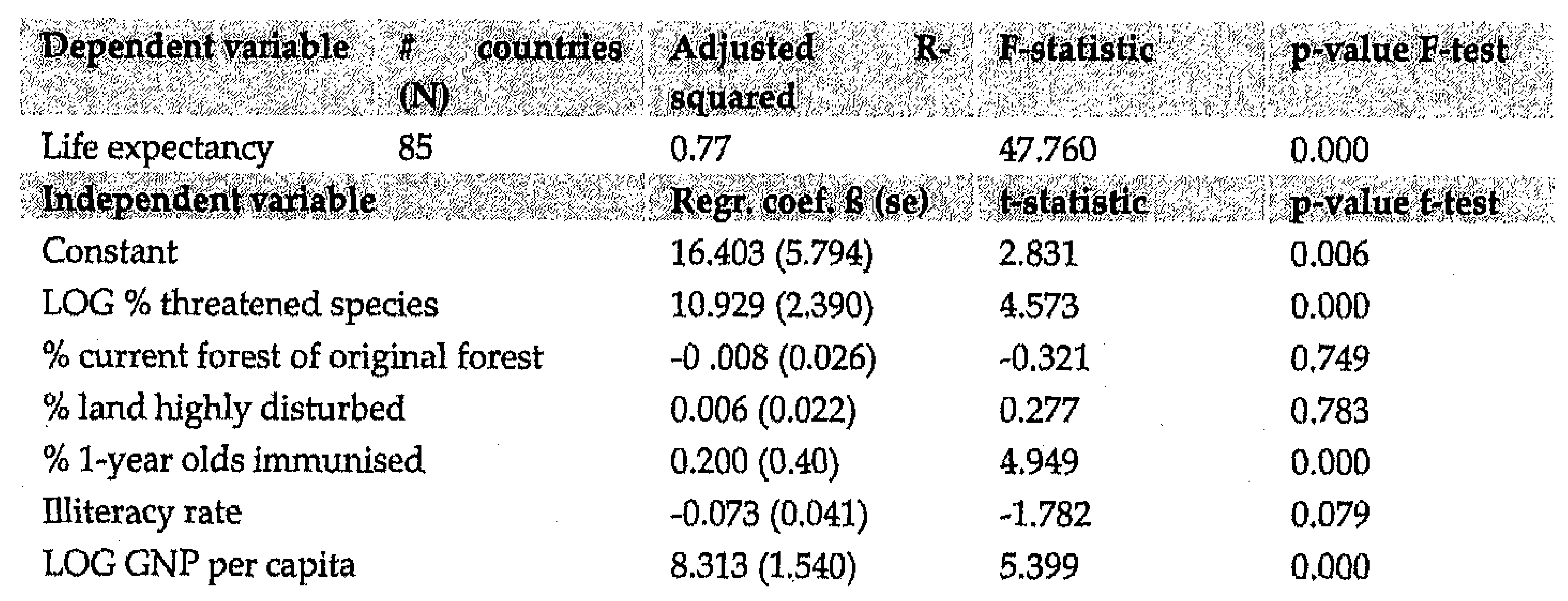




\section{Adjusted association between DALE and biodiversity}

Table 7.5 shows the regression results for DALE. The final model of DALE contained the following socio-economic variables: percentage one-year-olds immunised and the logarithm of GNP per capita. Health expenditure as percentage of GNP and illiteracy rate were deleted, because they had a nonsignificant association with DALE in this model.

Of the biodiversity indicators, only the logarithm of the percentage threatened species had a regression coefficient that was significantly different from zero; an increase in the logarithm of the percentage threatened species was significantly associated with an increase in DALE. Although the crude association between these two indicators was non-significant, the correlation was in the unexpected positive direction as well. The percentage highly disturbed land was almost significant with a $p$-value $(p=0.104)$ only slightly larger than our threshold value, but the association was not very strong.

The significant crude association between DALE and the percentage current forest of original forest disappeared after control for confounding in our model. However, when calculated based on the whole dataset (results not shown), both Log GNP per capita and percentage one-year olds immunised were not significantly correlated with this biodiversity indicator and, therefore, these socio-economic indicators seemed to be no confounders of the relationship between DALE and the percentage remaining forest. But these correlations are based on a larger number of countries than the regression model and, therefore, we recalculated the correlations within a database containing the same 117 countries as the regression model (results not shown). Based on this smaller database the crude positive association between DALE and the percentage remaining forest remained significant $(\mathrm{r}=0.184)$ and the percentage current forest of original forest was significantly positive correlated with Log GNP per capita $(r=0.163)$. Hence, the crude association between DALE and current forest as percentage of original forest is corrected in the model by controlling for Log GNP per capita.

Of the two social-economic indicators in the final model, the logarithm of GNP per capita had an association with DALE that was much stronger than the association between DALE and the percentage one-year olds immunised. 
Table 7.5: Results of the final regression model of Disability Adjusted Life Expectancy (DALE).

$\begin{array}{lllll}\text { Dependent variable } & \text { \# countries } & \text { Adjusted R- } & \text { F-statistic } & \text { p-value F-test } \\ & (\mathrm{N}) & \text { squared } & & \\ \text { DALE } & 117 & 0.790 & 88.201 & 0.000 \\ \text { Independent variable } & \text { Regr. coef. B (se) } & \text { t-statistic } & \text { p-value t-test } \\ \text { Constant } & -7.407(3.080) & -2.405 & 0.018 \\ \text { LOG\% threatened species } & 6.157(1.964) & 3.136 & 0.002 \\ \text { \% current forest of original forest } & 0.027(0.021) & 1.248 & 0.215 \\ \text { \% land highly disturbed } & 0.033(0.020) & 1.640 & 0.104 \\ \text { \% 1-year olds immunised } & 0.237(0.036) & 6.671 & 0.000 \\ \text { LOG GNP per capita } & 11.776(0.979) & 12.026 & 0.000\end{array}$

Adjusted associntion between infant mortality rate and biodiversity

Table 7.6 shows the regression results for infant mortality rate. The final model of the infant mortality rate contained the following socio-economic variables: the percentage one-year olds immunised, illiteracy rate and the logarithm of GNP per capita. Health expenditure as percentage of GNP was deleted, because it had a non-significant association with infant mortality rate in this model.

Of the biodiversity indicators in the regression model, not one was significantly associated with infant mortality rate. The significant crude associations between this health indicator and current forest as percentage of original forest respectively the percentage highly disturbed land are probably biased by socioeconomic confounding.

There was also a significant negative crude association between the percentage threatened species and infant mortality rate, but in the regression model this association disappears. It seems that this crude association was also biased by confounding. However, when calculated based on the whole dataset (results not shown), not one of the socio-economic variables was significantly correlated with this biodiversity indicator and, therefore, these socio-economic indicators seemed to be no confounders of the relationship between infant mortality rate and the percentage threatened species. However, these correlations are based on a larger number of countries than the regression model. We recalculated the correlations within a database containing the same 86 countries as the regression model (results not shown). Based on this smaller database the crude association between infant mortality rate and the percentage threatened species remained negative and significant $(r=-0.380)$ and the percentage threatened species was significantly correlated with all socio-economic variables in the model (percentage one-year olds immunised $(r=0.268)$; illiteracy rate $(r=-0.192)$ and Log GNP per capita $(r=0.319))$. The crude association between infant mortality rate and the percentage threatened species is corrected in the model by controlling for socio-economic development. 
Of the social-economic indicators in the final model, the logarithm of GNP per capita had the strongest association with infant mortality rate.

Table 7.6: Results of the final regression model of infant mortality rate.

\begin{tabular}{|c|c|c|c|c|}
\hline Dependent variable & $\begin{array}{l}\text { \# countries } \\
\text { (N) }\end{array}$ & $\begin{array}{l}\text { Adjusted R- } \\
\text { squared }\end{array}$ & F-statistic & p-value F-test \\
\hline Infant mortality rate & 86 & 0.770 & 48.298 & 0.000 \\
\hline \multicolumn{2}{|c|}{ Independent variable } & Regr. coef. B (se) & t-statistic & $p$-value t-test \\
\hline \multicolumn{2}{|c|}{ Constant } & $193.027(18.236)$ & 10.585 & 0.000 \\
\hline \multicolumn{2}{|c|}{ LOG $\%$ threatened species } & $-8.623(6.926)$ & -1.245 & 0.217 \\
\hline \multicolumn{2}{|c|}{$\%$ currents forest of original forest } & $0.002(0.082)$ & 0.019 & 0.985 \\
\hline \multicolumn{2}{|c|}{$\%$ land highly disturbed } & $-0.084(0.068)$ & -1.236 & 0.220 \\
\hline \multicolumn{2}{|c|}{$\%$ 1-year olds immunised } & $-0.360(0.128)$ & -2.822 & 0.006 \\
\hline \multicolumn{2}{|c|}{ Illiteracy rate } & $0.322(0.129)$ & 2.490 & 0.015 \\
\hline \multicolumn{2}{|l|}{ LOG GNP per capita } & $-36.105(4.719)$ & -7.650 & 0.000 \\
\hline
\end{tabular}

Adjusted association between low-birth weight and biodiversity

Table 7.7 shows the regression results for the percentage low-birthweight babies. The final model of percentage low-birthweight babies contained the following socio-economic variables: the percentage one-year-olds immunised and the logarithm of GNP per capita. Like development grade, illiteracy rate was deleted from this model to avoid multicolinearity with Log GNP per capita. Health expenditure as percentage of GNP was deleted, because it had a nonsignificant association with the percentage low-birthweight babies in this model.

Of the biodiversity indicators in the regression model, not one was significantly associated with the percentage low-birthweight babies. There was a significant crude association between this health indicator and percentage highly disturbed land, but the socio-economic confounders probably biased this crude association. The other biodiversity indicators showed no association with the percentage low-birthweight babies, with or without control for socio-economic development. Of the social-economic indicators in the final model, the logarithm of GNP per capita had the strongest association with the percentage low-birthweight babies. 
Table 7.7: Results of the final regression model of percentage low-birth-weight babies.

\begin{tabular}{|c|c|c|c|c|}
\hline Dependent variable & (N) countries & $\begin{array}{l}\text { Adjusted } \\
\text { squared }\end{array}$ & F-statistic & p-value F-test \\
\hline $\begin{array}{l}\text { Low-birth-weight } \\
\text { babies }\end{array}$ & 93 & 0.611 & 29.923 & 0.000 \\
\hline \multicolumn{2}{|l|}{ Independent variable } & Regr coef $B$ (se) & tostatistic & p-valuet-test \\
\hline \multicolumn{2}{|l|}{ Constant } & $30.124(1.862)$ & 16.174 & 0.000 \\
\hline \multicolumn{2}{|c|}{ LOG \% threatened species } & $0.383(1.090)$ & 0.352 & 0.726 \\
\hline \multicolumn{2}{|c|}{$\%$ currents forest of original forest } & $0.020(0.012)$ & 1.593 & 0.115 \\
\hline \multicolumn{2}{|c|}{$\%$ land highly disturbed } & $0.004(0.011)$ & 0.322 & 0.748 \\
\hline \multicolumn{2}{|c|}{$\%$ 1-year olds immunised } & $-0.050(0.022)$ & -2.329 & 0.022 \\
\hline \multicolumn{2}{|c|}{ LOG GNP per capita } & $-4.977(0.550)$ & -9.052 & 0.000 \\
\hline
\end{tabular}

\subsection{DISCUSSION AND CONCLUSIONS}

The comparison of the crude associations between biodiversity and health with the same associations corrected for confounding showed that several crude associations were indeed confounded by socio-economic development. After controlling for confounding, current forest as percentage of original forest and the percentage land highly disturbed by human activities both had no relationship with one of the health indicators.

We argue that biodiversity loss could result in compromised ecosystem functions, which, in turn, could negatively influence human health. Therefore, we expected that the relationship between the percentage of threatened species and human health to be negative. However, the positive crude association between the logarithm of the percentage threatened species and life expectancy respectively DALE remained after control for the selected socio-economic indicators. We were not able to explain these unexpected positive associations; perhaps unknown confounders bias these relationships or the percentage threatened species is not a good indicator to investigate the adverse health effects of biodiversity loss and the reduction in ecosystem functioning. Of the socio-economic indicators, the logarithm of GNP per capita had the strongest relationship with human health.

Sieswerda et al. (2001) had conducted a similar study investigating the link between life expectancy and measures of ecological (dis)integrity (percentage land highly disturbed by human activities, percentage forest remaining, percentage annual change in forest, Log percentage threatened species, Log percentage land totally protected land, Log percentage land partially protected), with control for Log GDP per capita. It is important to note that all three indicators that we selected for biodiversity were also among the six indicators Sieswerda et al. selected as measures of ecological integrity. Our regression 
model of life expectancy contained almost double the number of cases as the model of Sieswerda et al. and our database contained indicators for more recent years. They concluded that in their regression model no relationship existed between the selected measures of ecological integrity and life expectancy, but in our model the logarithm of the percentage threatened species showed an unexpected positive and significant association with this health indicator.

The present study was not able to provide any statistical proof of the expected negative association between loss of biodiversity and human health. There may be several reasons for our findings.

- Finding suitable indicators: We could only make use of available indicators, but these indicators are less able to reflect the true state of a highly complex concept (Soberon et al., 2000). The lack of correlation between the different biodiversity indicators indicates the difficulty and complexity of measuring biodiversity; obviously, the selected indicators do not measure the same thing. In combination with the fact that we still do not know which part of biodiversity is most important for human health, this makes it very difficult and time-consuming to collect suitable measures of biodiversity to investigate its link with human health. It is also important to note that under the CBD a provisional list of global indicators has been formulated in order to assess trends in biodiversity; these indicators are now being further developed and tested (CBD, 2007). In view of this need for sound biodiversity indicators, the 2010 Biodiversity Indicators (UNEP \& Global Environment Facility, 2007) was launched in July 2007 in order to 'complete a set of indicators that will allow the international community to better assess whether conservation efforts are succeeding towards the target of 'reducing the rate of loss of biodiversity by 2010'. However, these indicators will primarily be used for monitoring purposes and will not specifically be developed in order to asses the health impacts of biodiversity.

- Non-randomness in the selection of countries: The fact that the indicators chosen were not available for all countries resulted in a sample of countries that was not random, but depended on the availability of the required data; the sample, therefore, reflects conditions such as not having the infrastructure to collect data, being in an area to much affected by war to collect data, or, paradoxically, not being rich enough to collect one's own data, but not being poor enough to receive help from United Nations agencies (Sieswerda et al., 2001). The question remains to what extent this non-randomness in the selection of countries for the regression models affected the validity of the samples.

- Limitations of regression analysis: One has also to recognise the limitations of regression analysis in providing any proof of causality. It is based on correlations and these only indicate observed associations between the dependent and independent variables (corrected for other factors in multiple regression analysis). 
- Non-linearity: Our model assumed a linear relationship between reductions in biodiversity and human health and, consequently, this implies a linear relationship between loss of biodiversity and the provision of relevant ecosystem goods and services. There are, however, many uncertainties about the shape of the relationship between loss of biodiversity and reductions in ecosystem functioning. In the literature other possible shapes of this relationship is discussed (Schwartz et al., 2000), suggesting that ecosystems can lose parts of their biodiversity without consequences for their functioning. Only when a threshold in the losses of biodiversity is reached, the provision of ecosystem goods and services gets compromised; before this threshold is reached the reductions in biodiversity have hardly any effects on the functioning of the ecosystem and, as a consequence, on our health. When this is the correct shape of the real biodiversity-health relationship and most countries still have not reached their threshold, the losses in biodiversity have, at the moment, no demonstrable adverse healtheffects.

- Accounting for the population context: Socio-economic developments pose great pressures on biodiversity. It was, therefore, expected that socioeconomic development and loss of biodiversity were positively related. A problem with this expectation is, however, that the loss in biodiversity in one country is not per definition the result of social-economic developments in that particular country, but could also be the result of social-economic developments in other parts of the world (Wackernagel \& Rees, 1996). Sieswerda et al. (2001) argued that there is a 'separation of consumption from consequence'; many high-income countries attained wealth, first, at the expense of their own landscapes and biodiversity, and subsequently by using their wealth to exploit the ecological systems in other parts of the world. The crude associations between the biodiversity and socio-economic indicators showed no convincing pattern of an increasing loss of biodiversity with an increasing socio-economic development. Whether this is due to the phenomenon described above, the effect of confounding factors or other causes has to be further explored in future studies. We should find better ways to deal with the socio-economic population context.

- Multi-level system approach: It is also possible that the scale level of this study is not suitable for investigating the biodiversity-health relationship. Perhaps on a more local level, there are evidences of compromised health due to losses in biodiversity and the resulting reductions in ecosystem functioning (such as water purification by wetlands and air pollution reduction by vegetation), but these more local associations cannot be measured using data available at the country level. The integration of the different scale levels involved will probably be of vital importance.

To conclude, despite all the discussions in the literature about the adverse health effects of reductions in biodiversity, the present study was not able to 
provide any empirical proof of these expected negative health effects. It is not a simple matter of the direct effect of an exposure on a specific disease outcome; there may be several reasons for our findings, and we identified a number of aspects of the complex relationship between biodiversity and health that require further investigation in the upcoming years. Since the publication of this study in 2004, the number of publications stressing a systems approach towards 'biodiversity and health' is growing, partially influenced by insights from the ecological field. Our study, together with the work by Sieswerda et al. (2001), is mentioned in the MA (Chopra et al., 2005) to stress that 'the links between biodiversity loss and human health are difficult to demonstrate scientifically, due to the many factors that may confound such an association, difficulties in modelling nonlinear relationships, and lack of suitable data at appropriate scales'. In other words, the challenges encountered and the need for new innovative methodologies are perfectly illustrated by these 'linkage' studies. Increasingly, scientists talk in terms of 'ecosystem functions relevant for human health' rather than about the health effects of biodiversity loss. The recent MA (Carpenter et al., 2005) provides an integrated participatory scenario analysis of the changing ecosystem goods and services relevant for human well-being. Although the realisation that a systems approach is probably more suitable in order to study the health effects of declining ecosystem functioning is gaining ground, but not much empirical evidence is provided in the in the MA. The search for innovative research approaches, appropriate temporal and geographical scales, and suitable cases is still ongoing (Pongsiri \& Roman, 2007). Therefore, we hope that this paper will stimulate more comprehensive studies that will deal with the issues discussed above. 


\section{Chapter 8}

\section{CONCLUSIONS}




\section{CONCLUSIONS}

In light of the international commitment to sustainable development, exploring our future has become a matter of urgency and relevance. Studying the future of our health should be a vital part of this. In doing so, we need to recognise that the prospects for future health increasingly depend on the various processes of globalisation. An important objective of this Ph.D. Thesis is to provide a better understanding of the possible (future) health implications of the globalisation process, including global environmental change. This will aid both scientists and policymakers to work through the wide variety of interactions between global developments and human health, dealing with uncertainty, plurality, and interdisciplinarity along the way.

The central question underlying this study was as follows: How will future health evolve given anticipated globalisation trends? Population health is perceived as the integrated outcome of the economic, social-cultural, institutional, and ecological determinants that affect a population's physical, mental, and social abilities to function normally. Globalisation is defined as a process characterised by a growing intensity, extensity, and velocity of institutional, economic, socialcultural and ecological interactions, resulting in transborder processes and effects. In order to address this question, the following research objectives were identified:

1. To assess past developments in population health, both in the developed and developing world.

2. To develop an integrated framework to analyse the consequences of globalisation for population health, accounting for plurality along the way.

3. To explore future health given anticipated globalisation pathways and associated developments in important health determinants, both in the developed and developing world.

4. To explore the (future) health impacts of global environmental, focussing on global climate change and global biodiversity loss.

Furthermore, the methodological approach underlying this study is meant to contribute to the ongoing discussions and development of methods in the analysis of (future) health in a globalising world. This Chapter recapitulates the main conclusions drawn in this Ph.D. Thesis, provides a brief methodological reflection, and offers an outlook to future work. 


\subsection{PAST DEVELOPMENTS IN OUR HEALTH: THE EPIDEMIOLOGICAL TRANSITION}

Objective 1: To assess past developments in population health, both in the developed and developing world.

Although primarily future oriented, this study started with looking back to past developments in population health. An overview is given of the past developments in health using the framework of the epidemiological transition theory. The descriptive analysis of the changes observed in the Netherlands and Peru provided insights into the different transition experiences of these two countries. Previous studies already concluded that the past epidemiological changes in the Netherlands are a good example of the western transition model: population health developed from the 'age of pestilence and famine', through the 'age of receding pandemics', to the 'age of degenerative, stress, and manmade diseases'. However, these studies did not take the description of the fourth stage- the 'age of declining cardiovascular mortality, ageing, lifestyle modifications and resurgent diseases'- into account. Our analysis showed that Dutch epidemiological changes in the past decades (post-1970) clearly demonstrate that the Netherlands currently finds itself in this fourth stage. The non-western transition model describes three stages, of which the first two stages are named the same as in the western model, but differ in timing, pace and underlying processes. The third stage is called the 'age of triple health burden in non-western societies'. The changes in the Peruvian health situation described in this thesis are a good example of this non-Western transition model; Peru is now clearly in the third stage, with the country experiencing a triple health burden (i.e., facing both old and new health problems, while struggling with inadequate (access to) health services). A complicating factor for Peru is the wide range and inequity in health status within the country.

Subsequently, we assessed what we can learn from this epidemiological transition theory in order to improve our understanding of the broader context of population health. Since its first publication in 1971, the epidemiological transition theory has been extensively studied, but also debated. One key aspect in these discussions concerned the main driver(s) of the observed changes. Others have commented on the rather straightforward definition of the theory and stressed the risk of overgeneralisation. These debates as well as the two case studies provide some useful lessons for our exploration of future health. The original theory was based on a rather deterministic view of population health. However, not all countries follow the same transition pathway, and we should take into account that different (groups of) countries will differ in their future transition experience. Therefore, we need to make at least a distinction between future health in the developed and developing world. The epidemiological transition also shows that the system can respond in an 
unpredictable way. In the context of this Ph.D. Thesis, this implies that multiple health futures are possible (contrary to the mere extrapolation of business-asusual trends). Additionally, the causal mechanism behind the observed epidemiological changes cannot be explained using reductionist approaches. It is increasingly argued that not one or two main factors triggered the vast improvements in our health, but the combination of reinforcing developments. This is in line with our claim that a more integrated view of health determination -accounting for the broader context of population health- is fundamental to advance our understanding.

\subsection{GLOBALISATION AND HEALTH: A CONCEPTUAL FRAMEWORK}

Objective 2: To develop an integrated framework to analyse the consequences of globalisation for population health, accounting for plurality along the way.

As the pathways from globalisation to health are various and mediated by a multitude of factors, a clear conceptualisation of the system under investigation is required. We described the stepwise development of a new conceptual framework for the health implications of the globalisation process, building on a comprehensive analysis of relevant literature regarding population health models, the globalisation processes, and the various perspectives that abound in current literature regarding the effects of globalisation on population health. The multicausality of population health played an important role in the development of our conceptual framework for the health implications of globalisation. A review of existing health models formed the basis for the identification and structuring of the most important factors influencing health. The nature of the determinants (institutional, socio-cultural, economic, and environmental) and their level of causality (proximal, distal, and contextual) were combined into a basic framework that conceptualises the multi-causality of population health. An overview of the health determinants that can be fitted within this framework was given in Table 4.2 and Figure 4.2. In order to focus our conceptual framework, we distinguished-with the broader definition of globalisation in mind- the following important features of the globalisation process: new global governance structures, global markets, global communication and diffusion of information, global mobility, cross-cultural interaction, and global environmental changes. These features all operate at the contextual level of health determination, influence the distal factors identified within our framework, and- subsequently- the proximal health determinants. Accordingly, our conceptual framework linked these features of the globalisation process with the identified health determinants (Figures 4.3 and 4.4). The literature review underlying this study has been very extensive and 
the resulting framework reflects the diversity of views in various relevant disciplines.

Especially in the phase of issue- or problem-framing, the development of a conceptual model improves the integrated understanding of all key components and processes involved. This process should explicitly acknowledge the uncertainties and, consequent, plurality involved. We claim that our approach has several beneficial characteristics, compared to other frameworks. First, it is embedded in a holistic approach towards globalisation; we perceive globalisation as an overarching process in which simultaneously many different processes take place in many societal domains. In addition, the conceptual framework is embedded in a systems approach towards population health. As a result, our model explicitly visualises that globalisation affects the institutional, economic, social-cultural and ecological determinants of population health and that the globalisation process mainly operates at the contextual level, while influencing health through the more distal and proximal determinants. It clearly demonstrates that an interdisciplinary approach towards globalisation and health is required, which draws upon the knowledge from relevant fields such as, for example, medicine, epidemiology, sociology, political sciences, (health) education, environmental sciences and economics.

Hence, the framework provides valuable insights in how to organise the various factors involved in studying the health effects resulting from globalisation. Therefore, it could contribute to further (empirical) research by serving as a well-structured 'think-model' or 'concept map'. Within this study, the developed framework served as a useful tool to structure the developments described in scenarios by the Millennium Ecosystem Assessment (MA).

\subsection{FUTURE HEALTH IN A GLOBALISING WORLD}

Objective 3: To explore future health given anticipated globalisation pathways and associated developments in important health determinants, both in the developed and developing world.

Developments in population health are, of course, an ongoing process. So which health future lies ahead? Current literature shows a prevailing lack of consensus about the (future) health effects of globalisation. We argue that due to the complex interplay between the various factors within the global system, multiple future pathways are possible. Developing health scenarios can provide important insights into the diverse health effects of globalisation by describing a range of possible interactions, and their consequences. We explored future health according to different globalisation pathways- for both the developed and developing world- by combining insights from past health transitions, nine 
selected global scenarios studies' (with a total of 35 scenarios), and the conceptual framework discussed above.

The inclusion of health in past global scenario exercises has been limited. Based on a review of the selected scenarios studies, we concluded that a mere $23 \%$ of the selected scenarios describe health adequately and in an integrated way. Contrary to other past scenario exercises, however, exploring future well-being was one of the initial goals of the MA; all MA-scenarios describe health as a more or less integrated outcome of multiple developments. Still, there are some inconsistencies within the MA-reports, which probably arise from the lack of a clear conceptualisation of population health (determination).

Globalisation, on the other hand, is included as one of the most important drivers of future change in the selected studies. Looking at other developments that possibly affect our future health (e.g., food, water, environment, social change, equity, economic growth, technology), we see that many are rather well addressed in most scenarios. It, therefore, would have been possible to describe future developments in health as an outcome of these multiple health determinants. Given that health is regarded by many as one of the most important assets of the human life, why has there been so little effort in the past decade of scenario development to explicitly address human health? From the point of view of the global scenario community, exploring the potential health impacts of global changes poses a difficult challenge. From a public health point of view, exploration of these global, long term and complex risks to human health seems far removed from the tidy examples that abound in textbooks of epidemiology and public health research.

Two main approaches to develop health scenarios can be distinguished (Huynen, 2003). First, one could develop new integrated health scenarios from scratch. This would be, of course, very challenging, and it will be possible to make use of the expertise already available in the scenario community. The second approach builds on the outcomes of earlier studies and constitutes enriching existing global scenarios with a health component. For our study, we chose the second approach, in order to provide examples on how future health can be incorporated in existing scenarios.

\footnotetext{
1 The following nine scenario studies were selected: the scenarios by the Global Scenario Group (GSG) (Gallopin et al., 1997); Millennium Project scenarios by the United Nations University (Glenn \& Gordon, 1998); Global Scenarios 2000-2050 by the World Business Council for Sustainable Development (WBCSD) (1998); Which World? by Hammond (1998); Global trends 2015 by the National Intelligence Council (NIC) (2000); Special Report on Emission Scenarios by the International Panel on Climate Change (IPCC) (2000); World Water Scenarios by the World Water Council (Gallopin \& Rijsberman, 2000); Global Environmental Outlook 3 scenarios by the United Nations Environmental Program (UNEP) (2002); the Millennium Ecosystem Assessment (MA) scenarios (Carpenter et al., 2005; Corvalan et al., 2005).
} 
Table 8.1: Future health in a globalising world.

\begin{tabular}{|c|c|c|c|}
\hline $\begin{array}{l}\text { Globalisation } \\
\text { pathway }\end{array}$ & $\begin{array}{l}\text { Variant } \\
\text { (scenarios*) }\end{array}$ & $\begin{array}{l}\text { Future health in the } \\
\text { developed world }\end{array}$ & $\begin{array}{l}\text { Future health in the } \\
\text { developing world }\end{array}$ \\
\hline \multirow[t]{2}{*}{$\begin{array}{l}\text { Globalisation } \\
\text { with an } \\
\text { economic focus }\end{array}$} & $\begin{array}{l}\text { Low mitigation } \\
\text { capacity } \\
\text { (GEO3-MF) }\end{array}$ & $\begin{array}{l}\text { 'Age of medical } \\
\text { technology', eventually } \\
\text { risk of 'age of emerging } \\
\text { infectious diseases' } \\
\text { increases considerably. }\end{array}$ & $\begin{array}{l}\text { Gradual shift into the 'age } \\
\text { of emerging infectious } \\
\text { diseases'. }\end{array}$ \\
\hline & $\begin{array}{l}\text { High mitigation } \\
\text { capacity } \\
\text { (SRES A1 \& } \\
\text { MA-GO) }\end{array}$ & $\begin{array}{l}\text { 'Age of medical } \\
\text { technology', eventually } \\
\text { risk of 'age of emerging } \\
\text { infectious diseases' } \\
\text { increases. }\end{array}$ & $\begin{array}{l}\text { 'Age of chronic diseases'/ } \\
\text { 'age of emerging infectious } \\
\text { diseases'. }\end{array}$ \\
\hline \multirow[t]{3}{*}{$\begin{array}{l}\text { Globalisation } \\
\text { with a } \\
\text { sustainability } \\
\text { focus }\end{array}$} & $\begin{array}{l}\text { Rapid progress } \\
\text { (SRES-B1 \& } \\
\text { GEO3-SuF) } \\
\text { Slow progress }\end{array}$ & 'Age of sustained health'. & 'Age of sustained health'. \\
\hline & $-(\mathrm{GEO} 3-\mathrm{PF})$ & $\begin{array}{l}\text { - Progress towards the 'age } \\
\text { of sustained health' via } \\
\text { 'age of medical } \\
\text { technology', but } \\
\text { transition is not complete. }\end{array}$ & $\begin{array}{l}\text { - 'Age of chronic diseases'/ } \\
\text { 'age of medical } \\
\text { technology'; eventually } \\
\text { risk of 'age of emerging } \\
\text { infectious diseases' } \\
\text { increases. }\end{array}$ \\
\hline & $-(\mathrm{MA}-\mathrm{TG})$ & $\begin{array}{l}\text { - Rapid shift to 'age of } \\
\text { medical technology', } \\
\text { eventually risk of 'age of } \\
\text { emerging infectious } \\
\text { diseases' increases. }\end{array}$ & $\begin{array}{l}\text { - 'Age of medical } \\
\text { technology' /'age of } \\
\text { emerging infectious } \\
\text { diseases'. }\end{array}$ \\
\hline \multirow[t]{2}{*}{$\begin{array}{l}\text { Fragmentation } \\
\text { (retreat of } \\
\text { globalisation) }\end{array}$} & $\begin{array}{l}\text { Economic focus } \\
\text { (SRES-A2, } \\
\text { GEO3-SeF \& } \\
\text { MA-OS) }\end{array}$ & $\begin{array}{l}\text { 'Age of medical } \\
\text { technology'/'age of } \\
\text { emerging infectious } \\
\text { diseases'. }\end{array}$ & $\begin{array}{l}\text { Rapid shift into the 'age of } \\
\text { emerging infectious } \\
\text { diseases'. }\end{array}$ \\
\hline & $\begin{array}{l}\text { Sustainability } \\
\text { focus (SRES-B2 } \\
\text { \& MA-AM) }\end{array}$ & $\begin{array}{l}\text { Mixed results. Some will } \\
\text { progress towards the 'Age } \\
\text { of sustained health' } \\
\text { (possibly via the 'age of } \\
\text { medical technology), but } \\
\text { this transition is not } \\
\text { complete. Other lack } \\
\text { behind and shift into 'age } \\
\text { of emerging infectious } \\
\text { diseases'. }\end{array}$ & $\begin{array}{l}\text { Mixed results. Some will } \\
\text { progress towards the 'age } \\
\text { of sustained health' } \\
\text { (possibly via the 'age of } \\
\text { medical technology), but } \\
\text { this transition is not } \\
\text { complete. Other lack } \\
\text { behind and shift into 'age } \\
\text { of emerging infectious } \\
\text { diseases'. }\end{array}$ \\
\hline
\end{tabular}

Abbreviations: SRES $=$ Special Report on Scenarios (IPCC, 2000); GEO3= Global Environmental Outlook 3 (UNEP, 2002); MA = Millennium Ecosystem Assessment (Carpenter et al., 2005; Corvalan et al., 2005); MF=Markets First; $P F=$ Policy First; SeF= Security First; SuF= Sustainability First; GO=Global Orchestration; TG=Technogarden; OS=Order from Strength; AM= Adapting Mosaic. 
We linked the institutional, economic, social-cultural, and environmental developments described by three existing scenario studies (SRES, GEO3, MA) to a set of possible health futures ('age of emerging infectious diseases', 'age of medical technology', 'age of sustained health', and 'age of chronic diseases for non-western countries'. For the MA-scenarios, we used the conceptual model developed earlier to structure this exercise.

Beneath the diversity in scenario names and the narrative motivation for each storyline lies a common set of archetypal globalisation pathways: a globalising world with a market-oriented economic focus, a globalising world with a 'sustainability' focus, and a fragmented world. For each group two main variants can be identified. Apparently, there is some kind of 'consensus' about the variety of possible globalisation futures. Table 8.1 summarises which health futures evolve from the different globalisation pathways anticipated in existing scenarios. This analysis provides an integrated outlook on fundamentally different health futures for both the developed and developing world.

\subsection{HEALTH IMPACTS OF GLOBAL ENVIRONMENTAL CHANGE}

Objective 4: To explore the (future) health impacts of global environmental change, focussing on global climate change and global biodiversity loss.

Several global environmental issues are affecting the world's ecosystems and, consequently, the provision of ecosystem goods and services. The fourth objective focused specifically on this environmental dimension of globalisation, assessing the health impacts of climate change and biodiversity loss.

\subsubsection{Climate change and temperature-related mortality in the Netherlands}

We focused on the climate change-induced changes in temperature-related mortality in the Netherlands, using a combination of epidemiological approaches and scenario analysis. We found an expected V-like relationship between outdoor temperature and mortality, with an optimum average temperature level corresponding to the lowest point of the curve (i.e., lowest mortality). A time-series analysis showed that total mortality, cardiovascular mortality, respiratory mortality, and mortality among the elderly (65+) increased with temperatures above this optimum value (exposure to heat) and below this optimum (exposure to cold). Mortality from malignant neoplasms and mortality in the youngest age group (0-64 years) were less sensitive to changes in outdoor temperature. Subsequently, we used episode analysis to explore the impact of cold spells and heat waves on mortality. Mortality increased significantly during all of the heat waves studied, with an average 
total excess mortality $12.1 \%$, which was mostly attributable to the increase in respiratory mortality and mortality among the elderly. The average excess mortality during the cold spells was $12.8 \%$, which was mostly attributable to the increase in cardiovascular mortality and mortality among the elderly. The results concerning the forward displacement of deaths due to heat waves were not conclusive. We found no cold-induced forward displacement of deaths. It is, however, important to bear in mind that this study is based on a small number of heat waves and cold spells.

Based on the results from the time-series analysis, we assessed the annual changes in the proportion temperature-related deaths attributable to climate change in 2050 (KNMI'06 scenarios) under different acclimatisation assumptions. This analysis included total mortality, cardiovascular mortality, respiratory mortality, and mortality among those aged 65 years and over. For many temperate or cold regions, including the Netherlands, it has been stated that the decrease in cold-related mortality could counterbalance the increasing rates of heat-related mortality, even resulting in a reduction in mortality. However, Table 8.3 shows that according to our results temperature-related mortality in the Netherlands might decrease, remain unchanged or increase, depending on the climate change scenario and acclimatisation assumption.

It is important to note that our results for the Netherlands contrast with the recent IPCC (2007a) conclusion that climate change will virtually certain reduce cold-related mortality. Our results considering acclimatisation show that in the scenarios with and without changing air circulation patterns cold-related mortality increases or remains virtually unchanged, respectively. The assumption that acclimatisation is proportional to the change in temperature is based on an earlier study for the World Health Organization (WHO) by McMichael et al. (2004). However, despite the consensus that people will adjust to a warmer climate, we need to recognise that is still difficult to assess what future acclimatisation will look like in terms of magnitude and pace.

Given the uncertainties of social change, it is very challenging to assess how the interplay with socio-economic developments (e.g., adaptation, demographic changes) will play out. Therefore, the analysis does not pretend to yield precise results or definitive conclusions about the temperature-related mortality effects of climate change in the Netherlands. Its main objective is to provide an orderof-magnitude estimate of the effects, acknowledging the uncertainties involved and communicating the assumptions made in an explicit way. Our calculations did not account for changes in the sensitivity of people, or changes in population structure. Due to the difficulties regarding the modelling of future heat waves and cold spells under various climate change scenarios, our study did not specifically look at the climate change-induced changes in mortality due to episodes of extreme heat or cold. 
Table 8.3: Changes in the proportion temperature-related mortality in the Netherlands according to the different climate change scenarios and acclimatisation assumptions (2050 compared to 1990).

\begin{tabular}{|c|c|c|}
\hline $\begin{array}{l}\text { KNMI'06 } \\
\text { scenarios }\end{array}$ & Acclimatisation & Summary of results \\
\hline$G$ and $W^{*}$ & No acclimatisation. & $\begin{array}{l}\text { The proportion temperature-related } \\
\text { mortality declines. }\end{array}$ \\
\hline$G+$ and $W t^{* *}$ & No acclimatisation. & $\begin{array}{l}\text { The proportion temperature-related } \\
\text { mortality declines, but this decline is } \\
\text { smaller compared to } G \text { respectively } W \text {. } \\
\text { This is particular the case for respiratory } \\
\text { mortality, but not for cardiovascular } \\
\text { mortality. }\end{array}$ \\
\hline$G$ and $W$ & $\begin{array}{l}\text { Acclimatisation proportional } \\
\text { to change in annual average } \\
\text { temperature. }\end{array}$ & $\begin{array}{l}\text { The proportion temperature-related } \\
\text { mortality remains unchanged. }\end{array}$ \\
\hline $\mathrm{G}+$ and $\mathrm{W}+$ & $\begin{array}{l}\text { Acclimatisation proportional } \\
\text { to change in annual average } \\
\text { temperature. }\end{array}$ & $\begin{array}{l}\text { The proportion temperature related } \\
\text { mortality increases due to increasing heat- } \\
\text { related as well as cold-related mortality. }\end{array}$ \\
\hline$G$ and $W$ & $\begin{array}{l}\text { Acclimatisation proportional } \\
\text { to change in summer average } \\
\text { temperature. }\end{array}$ & $\begin{array}{l}\text { Same as with acclimatisation proportional } \\
\text { to change in annual average temperature. }\end{array}$ \\
\hline$G+$ and $W+$ & $\begin{array}{l}\text { Acclimatisation proportional } \\
\text { to change in summer average } \\
\text { temperature. }\end{array}$ & $\begin{array}{l}\text { The proportion temperature related- } \\
\text { mortality rises even more (compared to } \\
\text { acclimatisation proportional to change in } \\
\text { annual average temperature). }\end{array}$ \\
\hline
\end{tabular}

\subsubsection{Global loss of biodiversity and health}

Besides climate change, several other global environmental changes are also believed to adversely affect human health, of which the loss in biodiversity is one. The loss of the earth's biodiversity is often viewed as an emerging global environmental disaster, posing a threat to human health. Finding empirical proof of the negative health consequences of biodiversity loss could be very important to stimulate political efforts regarding the conservation of the planet's biodiversity. As per the study by Sieswerda et al. (2001), we argued that biodiversity loss could result in compromised ecosystem functions, which, in turn, could negatively influence human health. Therefore, we expected that the relationship between the biodiversity loss and human health to be negative. We quantitatively assessed the association between biodiversity loss and health by means of a regression analysis, with control for socio-economic developments. However, after controlling for confounding, current forest as 
percentage of original forest and the percentage land highly disturbed by human activities both had no relationship with one of the health indicators. The positive crude association between the logarithm of the percentage threatened species and life expectancy respectively Disability Adjusted Life Expectancy remained after control for the selected socio-economic indicators. We were not able to explain these unexpected positive associations; perhaps unknown confounders bias these relationships, or the percentage threatened species is not a good indicator to investigate the adverse health effects of biodiversity loss and the reduction in ecosystem functioning.

Hence, the present study was not able to provide any statistical proof of the expected negative association between loss of biodiversity and human health. There may be several reasons for our findings: lack of suitable indicators, possible non-randomness in the selection of countries, limitations of regression analysis, possible non-linearity of the relationship, difficulties regarding the role of socio-economic development (e.g., separation of consumption from consequence (Sieswerda et al., 2001)), and inappropriate scale level. In the literature, for example, other possible shapes of this relationship are discussed, suggesting a threshold above which no effects occur (Schwartz et al., 2000). Additionally, the loss in biodiversity in one country is not only the result of social-economic developments in that particular country, but also results from social-economic developments in other parts of the world. A multi-level approach, integrating the different scale levels involved, could also have been more suitable. So looking back, the rather reductionist approach in this analysis might not seem the most obvious choice. The challenges encountered and the need for new innovative methodologies are perfectly illustrated by this study. However, such an epidemiological approach was not that odd at the start of this study. Since, the number of publications stressing a systems approach towards 'biodiversity and health' is growing, partially influenced by insights from the ecological field. In 2005, the MA stated that 'approximately $75 \%$ of all emerging diseases are zoonotic (coming from animals), thus stressing the importance of further investigation of the role of biodiversity and ecological dynamics that are now recognised as central to disease prevention' (Hassan et al., 2005). However, the search for adequate integrated research approaches, appropriate temporal and geographical scales, and suitable cases is still ongoing (Pongsiri \& Roman, 2007).

\subsection{METHODOLOGICAL REFLECTION}

The methodological approach underlying this study is meant to contribute to the development of methods in the analysis of (future) health in a globalising 
world. Chapter 2 discussed that the complexities involved in assessing the health impacts of globalisation are characterised by:

- holism and systems thinking;

- uncertainty and plurality;

- inter- and transdisciplinarity.

Accordingly, we argued that the study of globalisation and health should be rooted in an integrated systems approach, addressing these issues. The field of Integrated Assessment (IA) provides such methodology. However, there is no such thing as a ready-made, one-size-fits-all IA-toolkit. This section discusses, in retrospect, how we addressed the above-listed methodological challenges within our research.

\subsubsection{Holism and systems thinking}

In Chapter 3, we argued that past changes in our health cannot be explained by one single main driver, but by the interaction of multiple developments. This recognition that developments in population health must be viewed within the broader system of health determinants formed the foundation for our conceptual model. We first identified and structured the most important health determinants, and only then started to think about how the processes of globalisation fitted within this framework. To our knowledge, this is the first time that a framework describing the health effects of globalisation actually started from such an integrated conceptualisation of population health. This approach improved the integrated understanding of all relevant components and processes involved and showed that the health impacts of globalisation are modified by multiple interacting factors, which cannot be studied in isolation from each other. Our study predominantly focussed on the relationships in the direction from globalisation to health. This does not mean, however, that globalisation is an autonomous process. One has to keep in mind that globalisation is influenced by many developments at the other levels. Although these associations were not included in our conceptual framework, our scenario analysis did (implicitly) account for the fact that the future of globalisation is determined by many interacting developments. The selected scenarios studies layed out different globalisation pathways consistent with the underlying narratives and storylines. As such, our study recognises that globalisation depends on the interplay between many developments, which together form the broader context of population health. It is important to note, though, that we did not further explore how the resulting pictures of future health could have fed back to the scenario narratives (e.g., through demographic developments or adaptation policies).

Regarding our quantitative analysis of the health effects of global environmental change, we can state that the temperature-related mortality 
effects are rather straightforward and epidemiological approaches are widely accepted. We combined the observed exposure-effect relationships with future climate change scenarios. Still, one needs to account for other relevant future developments within the system such as acclimatisation, socio-economic developments, adaptation (capacity), and demographic changes. We accounted for acclimatisation using different acclimatisation assumptions. However, a tension exists between recognising the various important factors involved and the data available for an integrated quantitative analysis. Within the INTARESE (Integrated Assessment of Health Risks of Environmental Stressors in Europe) project (www.intarese.org), we currently explore how to tackle these difficulties. Our assessment of the relationship between health and the loss of biodiversity took a traditional epidemiological approach.

\subsubsection{Uncertainty and plurality}

Any exploration of the future health effects of globalisation is surrounded by uncertainty and plurality; the underlying processes are not fully understood and they might behave in unpredictable ways. Our conceptual model identified the multiple processes and factors mediating the effects of globalisation on health. In doing so, we explicitly described the plurality of perspectives on uncertain relationships in current literature.

Our study, subsequently, showed that scenario analysis is a useful tool to deal with such uncertainties. Scenarios are usually developed in sets, in which each scenario explores the implications of different assumptions concerning uncertain relationships or uncertain developments in key drivers (e.g., globalisation). We described a first-order attempt to add a health dimension to existing global scenarios. As such, we explored future health according to given assumptions about uncertain globalisation pathways and consequent developments in distal and proximal health determinants. This implied a lower emphasis on prediction, but an accompanying greater emphasis on understanding of the processes involved.

Although there is now near-unanimous scientific agreement that our global climate is changing due to anthropogenic interference, uncertainties remain about the sensitivity of the climate system to changes in greenhouse gas concentrations, and about the future trajectories of those concentrations. To be able to deal with these uncertainties in future climate change, we explored four different climate change scenarios for the Netherlands developed by The Royal Meteorological Institute for the Netherlands (KNMI). In addition, we explored various assumptions regarding acclimatisation. A logical next step would be to include assumptions regarding other crucial uncertainties; for example, by formulating a set of possible adaptation assumptions. In assessing the health 
effects of biodiversity, it is still a challenge to adopt a system-based approach and to start exploring the main uncertainties involved.

\subsubsection{Inter- and transdisciplinarity}

Due to its multi-causality, population health transcends disciplinary boundaries. Additionally, globalisation is a multi-dimensional phenomenon, encompassing institutional, economic, social-cultural and ecological processes. Hence, there is a need to move beyond the boundaries of disciplinary research. By definition, a interdisciplinary study integrates insights from relevant disciplines into a more comprehensive understanding of the issue at stake (Newell, 2001). Transdisciplinary research moves beyond disciplines using new concepts and methods (Rosenfield, 1992). Developing an integrative framework is an important step in the process of inter- and transdisciplinary studies (Newell, 2001; Rosenfield, 1992). Our conceptual model is based on an extensive review of literature from various disciplines (e.g., social sciences, cultural sciences, environmental sciences, macroeconomics, public health) in order to assess the system under investigation from various angles. A logical next step would have been to discuss and evaluate this framework in a collaborative/participatory setting with scientists from several relevant fields and other relevant stakeholders. However, such participatory processes are both time and money consuming. Besides the formulation of our integrated conceptual model, we used scenario analysis for the integration of knowledge from different fields in consistent storylines, transcending disciplinary boundaries. Our study builds upon nine selected existing global scenario studies, which were all developed using participatory (multi-stakeholder) processes.

In our quantitative analysis of the changes in temperature-related mortality due to climate change, we combined our epidemiological results regarding exposure-effect relationships with the knowledge from climate scientists about the possible range of future climate change in the Netherlands. As already discussed, a next step would be to further elaborate this analysis by combining it with knowledge about the possible impacts of public health interventions (e.g., adaptation) and demographic change. Finally, the disciplinary approach in our assessment of the health effects of global biodiversity loss demonstrates the need for the integration of knowledge and methods from various disciplines (i.e., combining epidemiological approaches with ecological insights). 


\subsection{GLOBALISATION AND HEALTH: FUTURE OUTLOOK}

The health impacts of globalisation pose new challenges to science and policymakers. The main research question underlying this study asked how future health will evolve given anticipated globalisation trends. However, the answer to this question cannot be captured in the prediction of a single picture of future health. As a result, the focus of this Ph.D. Thesis was aimed at enhancing our understanding of the processes involved in order to better anticipate future risks as well as the opportunities provided to us by the globalisation process.

However, this research is only one of the first and pioneering steps in that direction. The previous section already discussed some proposed refinements concerning the work presented in this Ph.D. Thesis. This section offers a few general suggestions regarding future research lines to be included in the global health agenda. One important avenue for future research is to further develop integrated health scenarios, using a combination of suitable tools. With regard to the assessment of the health impacts of global environmental change, we underline the need for innovative system-based research approaches, as- for example- those proposed by the Earth System Science Partnership (ESSP) (Canfalonieri \& McMichael, 2006). Finally, we briefly point out that the international policy agenda also needs to reflect the reality that contemporary globalisation is an important health determinant.

\subsubsection{Developing global health scenarios: the road ahead}

Health is only beginning to play a role in global scenario assessments. Our study provides useful insights in how to deal with health in scenarios and shows that a comprehensive picture of future health evolves when all relevant social-cultural, institutional, economic, and environmental developments are taken into account. The MA (Carpenter et al., 2005) concludes that in the near future, enriching (global) scenarios - often in collaboration with key stakeholders- will define an important agenda for policy analysis, scientific research and education. This is certainly the case for the very relevant field of 'global health'. We believe that the current study provides an important step forward in exploring the (future) health impacts of globalisation. However, we are only at the beginning of an exciting journey towards integrated health scenarios. We have to realise that this expedition will be a difficult one as the road ahead is expected to be bumpy. Barriers will have to be overcome and important questions have to be answered (Huynen, 2003).

For example, various scales are involved ranging from the global to the local, but how can we better account for these cross-scale interactions? The idea of 'multi-scale' scenarios is rather new (Kok et al., 2006; van Asselt et al., 2005), but 
could be a very interesting approach in assessing the health impacts of globalisation for specific regions (e.g., Europe, Latin America) and countries (e.g., the Netherlands, Peru). Such scenarios will describe globalisation from a global perspective, but explore its implications at the regional level and, subsequently, its consequences at the local level through several country case studies.

The quantification of important determinants and health outcomes will complement the qualitative narratives of possible futures, but are we able to construct appropriate mathematical models that quantitatively simulate the interactions between globalisation and health? Hence, a logical next step would be the translation of our conceptual model into a mathematical model in order to compute quantitative estimates of possible health effects. However, in order to bridge the prevailing gap between the conceptual framework and the formulation of modelled scenarios, an alternative approach would be the combination of scenario development with qualitative modelling approaches such as fuzzy cognitive mapping (see e.g. Kosko (1985). In short, fuzzy cognitive maps (FCMs) are graph structures that allow hazy degrees of causality between related concepts and the incorporation of weighted causal influences, using imprecise or fuzzy linguistic expressions (e.g., 'small positive', 'moderate negative', 'strong positive'). It would be interesting to explore, how FCMs can be converted into interactive qualitative models that are able to complement narrative storylines.

Additionally, the involvement of various scientific disciplines and stakeholders could be a useful contribution to this research field, but how can they be engaged in scenario development? An interdisciplinary approach towards globalisation and health requires a closer engagement between disciplines and research initiatives organised around the health challenges posed by globalisation rather than around specific research disciplines. Additionally, stakeholder involvement can be used as a means of knowledge production (e.g., identification of prevailing perspectives on key uncertainties) and extended peer review (Funtowicz \& Ravetz, 1990, 1993; Valkering et al., 2006). For example, model inputs and results can be discussed and complemented in a participatory setting involving multiple stakeholders. Stakeholder analysis will be needed to identify a diversity of participants in order to ensure a rich level of input to the scenario analysis and a rich idea base from which scenarios can be developed. Various participatory techniques are available (see Chapter 2).

Hence, an integrated assessment of future health in a globalising world will be best supported by a combination of tools. Despite expected difficulties, developing health scenarios is certainly a worthwhile exercise. An integrated set of global health scenarios could provide a useful contribution to the ongoing discussions on the health effects of globalisation, and can help to stimulate scientists, governments and other stakeholders to take a more integrated approach towards global health in order to find ways to ensure good global 
health governance, a healthy environment and good health for the future world population.

\subsubsection{Assessing the health impacts of global environmental change}

As already discussed, further research is needed to understand how the balance of heat-related and cold-related mortality could change under different socioeconomic scenarios and climate projections (IPCC, 2007a). However, other health effects of climate change are more complex and multi-faceted, such as, for example, impacts on vector-born disease risk (e.g., climate change may alter the distribution of vector species, vector survival and the reproduction and maturation rate of the infective agents, while these relationships are mediated by complex changes in ecosystems, alterations regarding non-human host species, socio-economic developments and future human behaviour).

Little formal research has been carried out on the risks to human health for most other global environmental changes (Canfalonieri \& McMichael, 2006). The MA was one of the first international initiatives focusing on the current and future consequences of human-induced changes to ecosystems and biodiversity. Noteworthy is the fact that our study was mentioned in the MA (Chopra et al., 2005) to illustrate that 'the links between biodiversity loss and human health are difficult to demonstrate scientifically, due to the many factors that may confound such an association, difficulties in modelling nonlinear relationships, and lack of suitable data at appropriate scales'. At the moment, the search for innovative research approaches is still ongoing: the U.S. Environmental Protection Agency (EPA), for example, recently announced their new initiative supporting 'interdisciplinary research to characterise the mechanisms that link biodiversity and human health and to use this knowledge to develop integrative tools and approaches for quantifying and predicting these relationships' (Pongsiri \& Roman, 2007).

There is a growing need to better understand the multi-faceted and complex linkages between global environmental change and human health. Against this background the ESSP initiated a so-called joint project to increase, strengthen, and then support and coordinate the (now slowly evolving) international research network in relation to this topic. For an overview of possible future research lines, we refer to the science plan and implementation strategy developed by this joint ESSP project on 'Global Environmental Change and Health' (Canfalonieri \& McMichael, 2006), which explicitly stresses the need for system-based, interdisciplinary approaches. 


\subsubsection{Global health: a priority for the international agenda}

Although further research is required, the international agenda urgently needs to reflect the reality that the globalisation process is an increasingly important (contextual) health determinant. As argued throughout this Ph.D. Thesis, researchers investigating future global health need to think in terms of systembased approaches and multiple possible futures; the same holds, of course, for policymakers and society. We need to step away from business-as-usual attitudes, sectoral-based solutions and short-term remedies.

In all futures envisioned by the selected scenario studies, the developed and developing world influence each other. In the more optimistic scenarios, the pathway towards sustainable health result from the cooperation between developed and developing regions, facing economic, social and environmental challenges together. In other scenarios, the rich might experience improvements in their health, but sooner or later they are confronted with the negative developments in the developing world spilling on to the edges of their daily lives. The scenarios depicting a fragmented future, the developed countries eventually end up paying the bill for closing their eyes for the interplaying trends of economic decline, social instability, and environmental depletion in the developing world. Hence, a healthy future population is a common challenge for North and South. Additionally, our scenarios analysis shows that in the optimistic scenarios, the move towards sustainable health is spurred by a wide-ranging societal change rather than a top-down policy push, which is in line with the stronger sustainability stance describe in current literature (e.g., Robinson (2004) and Williams and Millington (2004).

Looking at current international policy efforts and commitments, improving health worldwide is an important part of the United Nations Millennium Declaration (UN, 2000) and associated Millennium Development Goals (MDGs) ${ }^{2}$ to be reached by 2015. Soskolne et al. (2007) argue that the MDGs could provide a platform from which to advance sustainable global health. The recent MDG Report 2007 (UN, 2007a) argues that there has been clear progress towards implementing the MDGs, but overall success is still far from assured. It is argued that rapid and large-scale progress is still feasible, but this will depend in large part on whether developed countries make good on their aid commitments. In the Millennium Declaration (UN, 2000), governments agreed that globalisation should become a positive force for all. However, there is also a need to consider the negative health impacts of the globalisation process. Hence, anticipating both the risks and opportunities provided by the

2 The eight MDGs are: 1) to eradicate extreme poverty and hunger; 2) to achieve universal primary education; 3) to promote gender equality and women empowerment; 4) to reduce child mortality; 5) to improve maternal health; 6) to combat HIV/AIDS, malaria, and other diseases; 7) to ensure environmental sustainability; and 8) to develop a Global Partnership for Development. 
globalisation process should be a vital part of the international effort to achieve the MDG. Other examples of global initiatives that need to account for the health effect of globalisation are Health for All (global strategy for health development advocated by the WHO) and Agenda 21 (the United Nations programme of action on sustainable development). The recent Global Health and Foreign Policy Initiative explicitly addresses the health impacts of globalisation. Under this initiative, Brazil, France, Indonesia, Norway, Senegal, South Africa, and Thailand issued the Oslo Ministerial Declaration in March 2007. In this statement, they acknowledge that health as a foreign policy issue needs a stronger strategic focus on the international agenda (Anonymous, 2007).

Another approach that can be used to stress the importance of 'globalisation and health' might be through the international human rights framework (Benatar, 1998; Huynen \& Martens, 2008). Under international human rights law, states have the legal obligation to respect, protect and fulfil human rights for all citizens. They have to ensure that their own policies do not impact negatively on the enjoyment of human rights in other countries, and that the activities of the international organisations of which they are a member are human rights-consistent (Smaller, 2005). The multi-causality of population health stressed within this Ph.D. Thesis shows that achieving a healthy globalised world concerns not only the right to health, but also requires the fulfilment of other important human rights upon which human health and wellbeing depend. In other words, several important human rights issues mediate the pathways from globalisation to health, such as the right to health care, food, water, and a healthy environment. In addition, the international community must stress the human rights obligations and responsibilities of both state and non-state actors (e.g., the World Trade Organization (WTO), and transnational corporations). Hence, the current debate about the pros and cons of globalisation must recognise that the forces of globalisation should be subject to moral and ethical considerations and should respect international legal standards and principles. A rights-based approach integrates the international human rights norms and principles into the plans, policies and processes of globalisation. As such, we can use human rights to provide a framework for discussion and international cooperation concerning the right to 'a healthy globalisation' for everyone (Huynen \& Martens, 2008).

\subsubsection{Epilogue}

'Global health' is a rather new research field. An increasing number of researchers are now broadening their research agendas to include global influences on health. In addition, new researchers- aware of the emerging importance of this topic- also enter the global health field. The small, though 
growing number of persons tackling this topic are embedded in (informal) networks such as the Global Forum on Health Research, and the Fulbright New Century Scholars 2001-2002 ('Challenges to Health in a Borderless World'). In addition, the past few years witnessed the launch of several new scientific journals addressing the global influences on human health, such as 'Globalization and Health', 'Global Public Health', and 'Ecohealth'. A next step is the recognition that much of the research needs to be conducted within a systems context. Global processes- and their health impacts- do not occur in isolation from one another. This means that there is an increasingly perceived need for the development of system-based approaches and research tools (e.g., modelling, scenario analysis), but also for an international commitment to integrated global health strategies (Canfalonieri \& McMichael, 2006).

Finally, global health concerns everybody's health: my health and your health, the health of the rich and the health of the poor, the health of our children and the health of our children's children. Against this background, I hope that the research and methodological challenges discussed in this Ph.D. Thesis will be seen as a small step forward towards a better understanding of (how to assess) future health in a globalising world. 


\section{REFERENCES}

ACCC (2006). Data from the Dutch Association of Comprehensive Cancer Centres. http://www.ikcnet.nl/. Retrieved July, 8, 2006.

Acheson, D. (1998). Independent inquiry into the inequalities in health. London: The Stationery Office.

Agis Groep (1999). U over gezondheidszorg. Utrecht: Agis groep.

Alberdi, J., Diaz, J., Montero, J. \& Miron, I. (1998). Daily mortality in Madrid community 1986-1992: Relationship with meteorological variables. Eur. J. Epidemiol., 14, 571578.

Albrecht, G., Freeman, S. \& Higginbothman, N. (1998). Complexity and human health: the case for a transdisciplinary paradigm. Culture, Medicine and Psychiatry, 22, $55-92$.

Allison, A.D. (1999). Multiple regression: a primer. Thousand Oaks: Pine Forge Press, Inc.

Amelung, B. (2006). Global (environmental) change and tourism: issues of scale and distribution. Maastricht: Amelung Publishers.

American Council for the United Nations University (2001). 2001 State of the Future (CD-ROM to accompany the print 2001 State of the Future by J.C. Glenn and T.J. Gordon). American Council for the United Nations University.

Anderson, T.W. \& Le Richie, W.H. (1970). Cold weather and myocardial infarction. The Lancet, 1, 292-296.

Anomynous (2001). Trading health care away? GATS, public services and privatisation. Dorset: Briefing 23. The Corner House.

Anonymous (2007). Oslo Ministerial Declaration- global health: a pressing foreign policy issue of our time. The Lancet, 369, 1373-1378.

Aramburu, C.E. (1995). Dinámica Demográfica y Politica de Pablación en el Perú. internet: www.alter.org.pe/pobdes/con05.htm. Lima: Paper presented at the IV Reunión Nacional sobre Población, April 26-29 1995, Ica, Peru. AMIDEP- USAIDUNFPA.

Archibugi, D. \& Michie, J. (1995). The globalisation of technology: a new taxonomy. Cambridge Journal of Economics, 19, 121-140.

Archibugi, D. \& Iammarino, S. (2000). Innovation and globalisation: evidence and implications. In: Chesnais, F., Ietto-Gilles, G. \& Simonetti (Eds.). European integration and global technology strategies. London: Routeledge.

Archibugi, D. \& Lundvall, B.A. (Eds.) (2001). The globalising learning economy. Oxford: Oxford University Press.

Balasubramaniam, K. (2000). Globalisation and liberalisation of health care services: WTO and General Agreement on Trade in Services. Issue paper prepared for the People's Health Assembly, Savar Bangladesh, 4-8 December 2000.

Ballester, F., Corella, D., Perez-Hoyos, S., Saez, M. \& Hervas, A. (1997). Mortality as a function of temperature; a study in Valencia, Spain, 1991-1993. International Journal of Epidemiology, 26, 551-561.

Bardalez, C. (2002). Salud de la poblacion. Economia y Sociedad, 44.

Bardalez del Aguila, C. (2001). Salud de Poblacion. In: CIES (Ed.). Politicas de Salud 20012006 (Vol. 5). Lima: Consorcio de investigacion economica y social (CIES). 
Barrett, R., Kuzawa, C.W., McDade, T. \& Armelagos, G.J. (1998). Emerging and Reemerging Infectious Diseases: The third epidemiologic transition. Annual Review of Anthropology, 27, 247-271.

Baum, F. (2001). Health, equity, justice and globalisation: some lessons from the People's Health Assembly. J Epidemiol Community Health, 55, 613-616.

Beaglehole, R. \& Yach, D. (2003). Globalisation and the prevention and control of noncommunicable diseases: the neglected chronic diseases of adults. The Lancet, 362, 903-908.

Ben-David, D. (2000). Trade, growth and disparity among nation. In: WTO (Ed.). Income Disparity and Poverty, World Trade Organization Special Study 5. Geneva: WTO publications.

Benatar, S. (1998). Global disparities in health and human rights" a critical commentary. Health Law and Ethics, 88(2), 295-300.

Bennet, P. (1999). Understanding responses to risk: some basic findings. In: Bennet, P. \& Calman, K. (Eds.). Risk Communication and Public Health. Oxford: Oxford University Press.

Bensimon, C. \& Benatar, S. (2006). Developing sustainability: a new metaphor for progress. Theoretical Medicine and Bioethics, 27, 59-79.

Berkman, L.F., Glass, T., Brisette, I. \& Seeman, T.E. (2000). From social integration to health: Durkheim in the new millennium. Social Science and Medicine, 51, 843857.

Bettcher, D.W., Yach, D. \& Guindon, G.E. (2000). Global trade and health: key linkages and future challenges. Bulletin of the World Health Organization, 78, 521-534.

Blaze Corcoran, P., Vilela, M. \& Roerink, A. (Eds.) (2005). Toward a sustainable world: the Earth Charter in action Amsterdam: KIT Publishers.

Bobadilla, J.L., Frenk, J., Frejka, T., Lozano, R. \& Stern, C. (1990). The epidemiologic transition and health priorities. In: Jamison, D.T., Mosley, W.H., Measham, A.R. \& Bobadilla, J.L. (Eds.). Disease control priorities in developing countries. Oxford: Medical Publications.

Bobadilla, J.L. \& Possas, C. (1993). Health policy issues in three Latin American countries: implications of the epidemiological transition. In: Gribble, J.N. \& Preston, S.H. (Eds.). The epidemiological transition: policy and planning Implications for developing countries Washington, DC: National Acadamy Press.

Bolarte Espinoza, J.L. (2006). La transición epidemiológica en el Perú y la inversión en salud. Boletín epidemiológico, 15, 368.

Bradley, D., Cairncross, S., Haines, A. \& Stephens, C. (2001). Health and sustainable development: International Institute for Environment and Development.

Braga, A.L.F., Zanobetti, A. \& Schwartz, J. (2002). The effect of weather on respiratory and cardiovascular deaths in 12 U.S. cities. Environ Health Perspect, 110(9), 859863.

Bresser, A.H.M., Berk, M.M., van den Born, G.J., van Bree, L., van Gaalen, F.W., et al. (Eds.) (2005). The effects of climate change in the Netherlands. Bilthoven Milieu en Natuur Planbureau (MNP).

Bretherton, C. (1996). Global Political Economy, Oxford: Blackwell publishers 1td. 
Briggs, D., Corvalan, C. \& Nurminen, M. (1996). Linknge methods for environment and health analysis. WHO/EHG/95.26. Geneva: World Health Organisation, Office of Global an Integrated Environmental Health.

Brundtland, G.H. (2002). World Summit on Sustainable Development: importance of health in economic development makes it a priority. BMJ, 325, 399-400

Brundtland, G.H. (Ed.) (1987). Our common future: The World Commission on Environment and Development. Oxford: Oxford University Press.

Butler, C. (2005). Peering into the fog: ecological change, human affairs and the future. Ecohealth, 2, 17-21.

Bwire, R., Nagelkerke, N., Keizer, S., Annee-van Bavel, J., Sijbrant, J., et al. (2000). Tuberculosis screening among immigrants in The Netherlands: what is its contribution to public health? The Netherlands Journal of Medicine 56, 63-71.

Caldwell, J.C. (1993). Health transition: the cultural, social and behavioural determinants of health in the third world. Social Science and Medicine, 2, 125-135.

Caldwell, J.C. (1998). Basic premises for health transition in developing countries. World Health Statistics Quarterly 51, 120-136.

Caldwell, J.C. (2001). Population health in transition. Bulletin of the World Health Organization, 79, 159-160.

Canfalonieri, U. \& McMichael, A.J. (Eds.) (2006). Global environmental change and human health: science plan and implementation strategy. Earth System Science Partnership (ESSP report no. 4).

Carder, M., McNamee, R., Beverland, I., Elton, R., Cohen, G.R., et al. (2005). The lagged effect of cold temperature and wind chill on cardiorespiratory mortality in Scotland. Occup Environ Med, 62, 702-710.

Carpenter, S., Pingali, P., Bennett, E. \& Zurek, M. (Eds.) (2005). Ecosystems and human well-being: scenarios. Washington D.C.: Island Press.

Castells, M. (1996). The rise of the network society. Oxford: Blackwell.

CBD (2007). Indicators. http://www.cbd.int/2010-target/framework/indicators.shtml. Retrieved September, 2, 2007.

Chan, N.Y., Stacey, M.T., Smith, A.E., Ebi, K.L. \& Wilson, T.F. (2001). An empirical mechanistic framework for heat-related Illness. Clim Res, 16, 133-143.

Chapin, F.S., Zavaleta, E.S., Eviners, V.T., Naylor, R.L., Vitousek, P.M., et al. (2000). Consequences of changing biodiversity. Nature, 405, 234-242.

Chopra, K., Leemans, R., Kumar, P. \& Simons, H. (Eds.) (2005). Ecosystems and human well-being: policy responses. Washington D.C.: Island Press.

CIA (2007). The world fact book 2007: Central Intelligence Agency.

Collins, T. (2003). Globalization, global health and access to health care. Int J Health Plann Manege, 18, 97-104.

Colwell, R.R. (2004). Biocomplexity and a new public health domain, Ecohealth, 1, 6-7.

Commission on the Future of Health Care in Canada (2002). Globalization and Canada's health care system. Policy dialogue No. 11. Vancouver: University of British Colombia.

Comrie, A. (2007). Climate change and human health. Geography Compass, 1, 325-339.

Corvalan, C., Hales, S. \& McMichael, A.J. (Eds.) (2005). Ecosystems and human wellbeing: health synthesis Geneva: A report of the Millennium Ecosystem Assessment published by the World Health Organization. 
Cueto, M. (2001). The return of epidemics: health and society in Peru during the twentieth century. Aldershot, UK: Ashgate.

Cunningham, R. (1996). Smoke and mirrors: the Canadian tobacco war. Canada: International Development Research Centre.

Curriero, F.C., Heiner, K.S., Samet, J.M., Zeger, S.L., Strug, L., et al. (2002). Temperature and mortality in 11 cities of the Eastern United States. Am J Epidemiol 155(80-87).

Dahlgren, G. \& Whitehead, M. (1991). Policies and strategies to promote social equity in health. Copenhagen: World Health Organisation.

Daily, G. (1997). Nature's services: social dependence on natural ecosystems. Washington D.C.: Island Press.

Davis, R.E., Knappenberger, P.C., Novicoff, W.M. \& Michaels, P. (2003). Decadal changes in summer mortality in U.S. cities. Int J Biometeorol 47, 166-175.

de Beer, J. \& Harmsen, C. (2003). Ruim duizend doden extra door warme zomer. Statistics Netherlands Webmagazine(September 8).

de Groot, R.S. (1992). Functions of nature. Groningen: Wolters-Noordhof.

de Groot, R.S., van der Perk, J., Chiesura, A. \& Marguliew, S. (1999). Ecological functions and socio-economic values of critical natural capital as a measure for ecological integrity and environmental health. Paper prepared for the NATO Advanced Workshop on 'Implementing Ecological Integrity: Restoring Regional and Global Environmental and Human Health, National Institute of Health, Budapest, June 26 - July 11999.

de Groot, R.S., Wilson, M.A. \& Boumans, R.M.J. (2002). A typology for the classification, description and valuation of ecosystem functions, goods and services. Ecological Economics, 41, 393-408

de Hollander, A.E.M., Hoeymans, N., Melse, J.M., van Oers, J.A.M. \& Polder, J.J. (2006). Zorg voor gezondheid - Volksgezondheid Toekomst Verkenning 2006 [ Public Health Forecast 2006 ] Bilthoven: RIVM.

Deacon, B. (1997). Global social policy: international organizations and the future of welfare. London: Sage.

den Boon, S. \& van Pelt, W. (2003). Verdubbeling consulten voor tekenbeten en ziekte van Lyme. Infectieziekten Bulletin, 14(5), 162-163.

den Boon, S. \& van Pelt, W. (2006). De ziekte van Lyme in Nederland tussen 1994 en 2005: drievoudige toename van het aantal huisartsconsulten en verdubbeling van het aantal ziekenhuisopnames. Infectieziekten Bulletin, 17(7), 238-240.

den Draak, M. (2003). Early life changes: transition in pregnancy and birth outcome in South India. Amsterdam: Rozenberg Publishers.

Dessai, S.R. (2003). Heat stress and mortality in Lisbon Part II: an assessment of the potential impacts of climate change. Int J Biometeorol, 48, 37-44.

Diaz, J., Garcia, R., Lopez, C., Linares, C., Tobias, A., et al. (2005). Mortality impact of extreme winter temperatures, Int I Biometeorol 49, 179-183.

Dodgson, R., Lee, K. \& Drager, N. (2002). Global health governance: a conceptual review. London: Centre on Global Change and Health, London School of Hygiene and Tropical Medicine.

Dollar, D. \& Kraay, A. (2001). Growth is good for the poor. Policy research working paper No 2587. Washington, DC: World Bank. 
Donaldson, G.C. \& Keatinge, W.R. (1997). Early increases in ischemic hearth disease mortality dissociated from and later changes associated with respiratory after cold weather in South East England. Jounal of Epidemiology and Community Health, 51, 643-648.

Donaldson, G.C., Keatinge, W.R. \& Näyhä, S. (2003). Changes in summer temperature and heat-related mortality since 1971 in North Carolina, South Finland, and Southeast England. Environ Res 91, 1-7.

Duda, A. \& El-Ashry, M. (2000). Addressing the Global Water and Environment Crisis through Integrated Approaches to the Management of Land, Water and Ecological Resources. Water International, 25, 115-126.

Dunn, H. (1977). High-level wellness. Thorofare, New Jersey: Charles B. Slack, Inc.

Ebi, K.L., Tesiberg, T.J., Laklstein, L.S., Robinson, L. \& Weiher, R. (2004). Heat watch/warning systems save lives: estimated costs and benefits for Philadelphia 1995-98. American Meteorological Society, August, 1067-1073.

El - Zein, A., Tewtel - Salem, M. \& Nehme, G. (2004). A time-series analysis of mortality and air temperature in Greater Beirut. Science of the Total Environment 330, 71-80.

Eng, H. \& Mercer, J. (1998). Seasonal variations in mortality caused by cardiovascular diseases in Norway and Ireland. J Cardiovasc Risk, 5, 89-95.

Erkens, C.G.M. \& Veen, J. (2006). Neemt het anntal mensen met tuberculase toe of af? Volksgezondheid Toekomst Verkenning: Nationaal Kompas Volksgezondheid http://www.nationaalkompas.nl. Retrieved July, 17, 2006.

Eurowinter Group (1997). Cold exposure and winter mortality from ischemic heart disease, cerebrovascular disease, respiratory disease, and all causes in warm and cold regions of Europe. The Lancet, 349, 1341-1346.

Evans, R.G. \& Stoddart, G.L. (1990). Producing health, consuming health care. Soc Sci $\operatorname{Med}(31), 1347-1363$.

Ezzati, M. \& Lopez, A.D. (2001). Estimates of global mortality attributable to smoking in 2000. The Lancet, $362,847-852$.

FAO (1996). Food and international trade. The World Food Summit technical background document No. 12. Rome: Food and Agricultural Organisation of the United Nations.

FAO (2003). The state of food insecurity in the world 2003. Rome: Food and Agricultural Organisation of the United Nations.

FAO (2005). The state of food insecurity in the world 2005. Rome: Food and Agricultural Organisation of the United Nations.

Faulkner, B. (2001). The future ain't what it used to be. Gold Coast: Griffith University.

Feachem, R.G.A. (2001). Globalisation is good for your health, mostly. BMJ, 323, 504-506.

Fidler, D. (2002). Global health governance: overview of the role of international law in protecting and promoting global public health. Discussion paper no. 3. London: Centre on Global Change and Health, London School of Hygiene and Tropical Medicine.

Fidler, D. (2004). Germs, governance, and global public health in the wake of SARS. I Clin Invest, 113, 799-804.

Filozof, C., Gonzalez, C., Sereday, M., Mazza, M. \& Braguinsky, J. (2001). Obesity prevalence and trends in Latin-American countries. Obesity Reviews, 2, 99-106. 
Fischer, P.H., Brunekreef, B. \& Lebret, E. (2004). Air pollution related deaths during the 2003 heat wave in the Netherlands. Atmospheric Environment, 38, 1083-1085

Folke, C., Holling, C.S. \& Perrings, C. (1996). Biological diversity, ecosystems and the human scale. Ecological Applications, 6, 1018-1024.

Fox, J. (1997). Applied regression analysis, linear models, and related methods. Thousand Oaks: Sage Publications.

Frankel, J.A. \& Romer, D. (1999). Does trade cause growth? American Economic Review, June, 379-399.

Frankish, C.J., Green, L.W., Ratner, P.A., Chomik, T. \& Larsen, C. (1996). Health impact assessment as a tool for population health promotion and public policy. A Report submitted to the health promotion development division of health Canada. Vancouver: Institute of Health Promotion Research, University of British Columbia.

Frenk, J., Bobadilla, J.L., Sepulveda, J. \& Lopez-Cervantes, M. (1989). Health transition in middle-income countries: new challenges for organisation of services. Health Policy and Planning, 4, 29-39.

Frenk, J., Bobadilla, J.L., Stern, C., Frekja, T. \& Lozano, R. (1991). Elements for a theory of the health transition. Health Transition Review, 1, 21-38.

Frenk, J., Bobadilla, J.L. \& Lozano, R. (1996). The epidemiologic transition in Latin America. In: Imaeus, I.M., Chackiel, J. \& Rozicka, L. (Eds.). Adult mortality in Latin America. Oxford: Claredon Press.

Frenk, J., Sepulveda, J., Gomez-Dantes, O., McGuinnes, M.J. \& Knaul, F. (1997). The future of world health: the new world order and international health. BMJ, 314, 1404-1407.

Funtowicz, S.O. \& Ravetz, J.R. (1990). Uncertainty and quality in science. Dordrecht: Kluwer Academic Publishers.

Funtowicz, S.O. \& Ravetz, J.R. (1993). Science for the Post-Normal age. Futures, 25, 735755.

Funtowicz, S.O. \& Ravetz, J.R. (1994). Uncertainty, complexity and post-normal science. Environmental Toxicology and Chemistry, 13, 1881-1885.

Gage, T.B. (2005). Are modern environments really bad for us? Revisiting the demographic and epidemiologic transitions. Yearbook of Physical Antropology, 48(96-117).

Gallopin, G., Hammond, A., Raskin, P. \& Swart, R. (1997). Branch points: global scenarios and human choice. A resource paper of the Global Scenario Group. Stockholm: Stockholm Environment Institute.

Gallopin, G. \& Rijsberman, F. (2000). Three global water scenarios. International Journal of Water, 1, 16-40.

Garfield, M. \& Neugut, A. (1997). The human consequences of war:. In: Levy, B.S. \& Sidel, V.W. (Eds.). War and public health (pp. 27-38). New York: Oxford University Press.

Gaylin, D.S. \& Kates, J. (1997). Refocusing the lens: epidemiologic transition theory, mortality differentials, and the AIDS pandemic. Social Science and Medicine, 44, 609-612.

Gezondheidsraad (2002). Enkele belangrijke ontwikkelingen in de voedselconsumptie. $\mathrm{Nr}$ 2002/12. Den Haag: Gezondheidsraad Commissie Trends Voedselconsumptie. 
Gleick, J. (1987). Chaos: making a new science. London: Heineman.

Gleick, P.H. (2000). The world's water 2000-2001: the biennial report on freshwater resources. Washington, D.C.: Island Press.

Gleick, P.H. (2002). The world's water 2002-2003: the biennial report on freshwater resources. Washington, D.C.: Island Press.

Glenn, J.C. \& Gordon, T.J. (Eds.) (1998). 1998 State of the future: issues and opportunities. Washington: American Council for the United Nations University.

Gough, C., Castells, N. \& Funtowicz, S. (1998). Integrated Assessment: an emerging methodology for complex issues. Environmental Modeling and Assessment, 3, 1929.

Greeuw, S.C.H., van Asselt, M.B.A., Grosskurth, J., Storms, C.A.M.H., Rijkens-Klomp, N., et al. (2000). Cloudy crystal balls: an assessment of recent European and global scenario studies and models. Environmental issue report No 17. Copenhagen: European Environmental Agency.

Grifo, F. \& Rosenthal, J. (Eds.) (1997). Biodiversity and human health. Washington, D.C.: Island Press.

Grize, L., Huss, A., Thommen, O., Schindler, C. \& Braun-Fahrländer, C. (2005). Heat wave 2003 and mortality in Switzerland. Swiss Med Wkly, 135, 200-205.

Grosskurth, J. \& Rotmans, J. (2004). The SCENE-model: getting a grip on sustainable development. Environment, Development and Sustainability, 7, 133-149.

Grundy, E. (2005). The McKeown debate: time for burial. International Journal of Epidemiology, 34, 529-533.

Gupta, Y. \& van Asselt, H. (Eds.) (2004). Re-evaluation of the Netherlands' long-term climate targets. Amsterdam: Institute for Environmental Studies.

Hales, S. \& Woodward, A. (2003). Climate change will increase demands on malaria control in Africa. The Lancet, 362, 1775-1776.

Hammond, A. (1998). Which World? Scenarios for the 21st century: global destinies, regional choices. London: Earthscan.

Hancock, T. \& Perkins, F. (1985). The mandala of health: a conceptual model and teaching tool. Health Promotion, 24, 8-10.

Hancock, T. (1993). Health, human development and the community ecosystem: three ecological models. Health Promotion International, 8(1), 41-47.

Hannertz, U. (1996). Transnational connections: cultures, people, places. London: Routeledge.

Harremoës, P. \& Turner, R.K. (2001). Methods for integrated assessment. Regional Environmental Change, 2, 57-65.

Harris, B. (2004). Public health, nutrition, and the decline of mortality: the McKeown thesis revisited. Social History of Medicine, 17, 379-407.

Harris, G. (2002). Integrated assessment and modelling: an essential way of doing science. Environmental Modelling and Software, 17, 201-207.

Hassan, R., Scholes, R. \& Ash, N. (Eds.) (2005). Ecosystems and Human Well-being: current state and trends, Volume 1. Washington D.C.: Island Press.

Held, D., McGrew, A., Goldblatt, D. \& Perraton, J. (1999). Global transformations: politics, economics and culture. Stanford: Stanford University Press.

Held, D., McGrew, A., Goldblatt, D. \& Perraton, J. (2000). Rethinking globalisation. In: Held, D. \& McGrew, A. (Eds.). The global transformations reader. Cambridge: Polity. 
Hemmingway, H. \& Marmot, M. (1999). Evidence based cardiology: Psychosocial factors in the aetiology and prognosis of coronary hearth disease: systematic review of prospective cohort studies. BMJ, 318, 1460-1467.

Hodges, J.R. \& Kimball, A.M. (2005). The global diet: trade and novel infections. Globalization and Health, 1, 4.

Hoekstra, A.Y. \& Huynen, M.M.T.E. (2002). Balancing the world water supply and demand. In: Martens, P. \& Rotmans, J. (Eds.). Transitions in a globalising world. Lisse: Swets \& Zeitlinger B.V.

Hoekstra, A.Y. \& Hung, P.Q. (2005). Globalisation of water resources: international virtual water flows in relation to crop trade. Global Environmental Change, 15, 4556.

Hofstee, E.W. (1981). Korte demografische geschiedenis van Nederland van 1800 tot heden. Haarlem: Fibula-van Dishoeck.

Holm, H.H. \& Sorensen, G. (1995). Whose world order? Uneven globalization and the end of the cold war. Boulder: Westview Press.

Hong, E. (2000). Globalisation and the impact on health: a third world view. Issue paper prepared for The Peoples' Assembly, December 4-8, 2000, Savar Bangladesh.

Hoogeboezem, J. \& Garssen, J. (2006). Kanker bij mannen nu doodsoorzaak nr.1. Centraal Bureau voor de Statistiek Webmagine http://www.cbs.nl/nl$\mathrm{nl} / \mathrm{menu} /$ themas/mens-maatschappij/bevolking/publicaties/artikelen/2006-1953wm.htm. Retrieved July, 3, 2006.

House, J.S., Landis, K.R. \& Umberson, D. (1988). Social relations and health. Science, 241, 540-545.

Hulshof, K.F.A.M., Ocke, M.C., Rossum van, C.T.M., Buurma-Rethans, E.J.M., Brants, H.A.M., et al. (2004). Results of the national food consumption survey 2003 (in Dutch). Bilthoven: National Institute for Public Health and the Environment (RIVM).

Huntington, S.P. (1993). The clash of civalizations. Foreign Affairs, Summer 1993, 22-49.

Huynen, M.M.T.E., Martens, P., Schram, D., Weijenberg, M.P. \& Kunst, A.E. (2001). The impact of heat waves and cold spells on mortality rates in the Dutch population. Environmental Health Perspectives, 109(5), 463-470.

Huynen, M.M.T.E. \& Martens, P. (2002). Future health: the health dimension in global scenarios. Maastricht: ICIS.

Huynen, M.M.T.E. (2003). Scenarios and global health: the road ahead. IHDP Newsletter, issue $3 / 2003,14$.

Huynen, M.M.T.E., Martens, P. \& de Groot, R.S. (2004). Linkages between biodiversity loss and human health: a global indicator analysis. International Journal of Environmental Health Research, 14, 13-30.

Huynen, M.M.T.E., Vollebregt, L., Martens, P. \& Benavides, B.M. (2005a). The epidemiologic transition in Peru. Pan American Journal of Public Health, 17(1), 5159.

Huynen, M.M.T.E., Martens, P. \& Hilderink, H.B.M. (2005b). The health impacts of globalisation: a conceptual framework. Globalization and Health, 1, article number 14 (12 pages). 
Huynen, M.M.T.E., Martens, P. \& Hilderink, H.B.M. (2005c). The health impacts of globalisation: a conceptual framework. Bilthoven: Netherlands Environmental Assessment Agency (MNP-RIVM).

Huynen, M.M.T.E. \& Martens, P. (2006). Globalization and human health: toward scenarios for the 21st century. In: Interactions between Global Change and Human Health. Vatican: The Pontifical Academy of Sciences.

Huynen, M.M.T.E. (2006). The health effects of climate change in the Netherlands. In: Gupta, J. \& van Asselt, H. (Eds.). Assessing dangerous climate impacts for the Netherlands. Bilthoven: Netherlands Environmental Assessment Agency.

Huynen, M.M.T.E., Martens, P. \& Hilderink, H.B.M. (2007). The diverse pathways from globalization to health. In: Global Forum Update on Research for Health Volume 4. Equitable access: research challenges for health in developing countries London: ProBrook Publishing for the Global Forum on Health Research.

Huynen, M.M.T.E. \& Martens, P. (2008). Linkages among globalization, human rights, and health. In: Soskolne, C., Westra, L., Kotze, L., Mackey, B., Rees, W., et al. (Eds.). Sustaining life on earth: environmental and human health through global governance. Lanham: Lexington Books.

IMF (2004). Evaluation of the IMF's role in Poverty Reduction Strategy Papers and the Poverty Reduction and Growth Facility. Washington D.C.: International Monetary Fund.

INEI (2000). Las estadisticas vitales en los distritos del Perú. Lima: Perú, Instituto Nacional de Estadística e Informática.

INEI (2001). Peru: Compendio Estadistico, 2001. Lima: Perú, Instituto Nacional de Estadística e Informática.

INEI (2004). Data on the number of health establishments, http://www.inei.gob.pe. . Retrieved January, 24, 2004.

IOM (2003). The Future of Public Health in the 21st Century. Washington D.C.: Institute of Medicine. Committee on Assuring the Health of the Public in the 21st Century, Board on Health Promotion and Disease Prevention. The National Academy Press.

IPCC (2000). Special report on emission scenarios. Cambridge: Cambridge University Press.

IPCC (2001a). Climate change 2001: impacts, adaptation and vulnerability. Cambridge: Cambridge University Press.

IPCC (2001b). Climate change 2001: the scientific basis. Cambridge: Cambridge University Press.

IPCC (2007a). Climate change 2007: impacts, adaptation and vulnerability. Cambridge: Cambridge University Press.

IPCC (2007b). Climate change 2007; the scientific basis. Cambridge: Cambridge University Press.

Izsak, J. \& Papp, L. (2000). A link between ecological diversity indices and measures of biodiversity. Ecological Modelling, 130, 151-156.

Jager-Geurts, M.H., Peters, R.J.G., van Dis, S.J. \& Bots, M.L. (2006). Hart-en Vaatziekten in Nederland, 2006. Den Haag: Nederlandse Hartstichting.

Jernigan, D.H. (1997). Thirsting for markets: the global impact of corporate alcohol. Marin County: Marin Institute for the prevention of alcohol and other drug problems.

Kane, C.F. (1988). Family social support: toward a conceptual model. ANS-Advances in Nursing Science, 10, 18-15. 
Kapan, D.D., Bennet, S.N., Ellis, B.N., Fox, J., Lewis, N.D., et al. (2006). Avian Influenza (H5N1) and the evolutionary and social ecological basis for understanding emerging infectious disease risk. EcoHealth, 3, 187-194.

Kaul, I., Grumberg, Y. \& Stern, M.A. (Eds.) (1999). Global public goods: international cooperation in the 21st century. New York: Oxford university Press (for the United Nations development programme).

Keatinge, W.R. \& Donaldson, G.C. (1995). Cardiovascular mortality in winter. Artic Medical Research, 54: Suppl. 2, 16-18.

Keatinge, W.R., Donaldson, G.C., Cordioli, E., Martinelli, M., Kunst, A.E., et al. (2000). Heat related mortality in warm and cold regions of Europe: observational study. BMJ, 321, 670-673.

Keatinge, W.R. \& Donaldson, G.C. (2001). Mortality related to cold and air pollution in London after allowance for effects of associated weather patterns. Environ Res 86: Section A, 209-216.

Keohane, R.O. \& Nye Jr., J.S. (1977). Power and ioterdependence: world politics in transition. Boston: Little, Brown and Co.

Klaassen, A., Manger Cats, V., Heshusius, M. \& van der Wall, E. (Eds.) (2004). Een eeuw hart en vaatziekten in Nederland. Zwolle: Waanders.

KNMI (2007). The KNMI transformation and climate scenarios pages. http:/climexp.knmi.nl/Scenarios_monthly/ and http://www.knmi.nl/klimaatscenarios/knmi06/index.html. Retrieved August 4, 2007.

Knorr Cetina, K. (2005). Complex Global Microstructures: The New Terrorist Societies Theory, Culture \& Society 22, 213-234.

Koedijk, F., op de Coula, E.L.M. \& van de Laar, W.J.M. (2005). Aangifte acute hepatitis B in 2004 Infectieziekten Bulletin, 16, 296-298.

Kok, K., Biggs, R. \& Zurek, M. (2006). Methods for developing multiscale participatory scenarios: insights from southern Africa and Europe. Ecology and Society, 13, 8 (16 pages).

Kosko, B. (1985). Fuzzy cognitive maps. Int. J. Man-Machine Studies, 24, 65-75.

Kovats, R.S., Bouma, M.J., Hajat, S., Worral, E. \& Haines, A. (2003). El Niño and health The Lancet, 362, 1481-1489.

Kovats, R.S. \& Ebi, K.L. (2006). Heatwaves and public health in Europe. European Journal of Public Health, 16(6), 592-599.

Krzanowski, W.J. (1998). An introduction to statistical modelling. New York: Oxford University Press.

Kunitz, S.J. (1990). The value of particularism in the study of the cultural, social and behavioural determinants of mortality. In: Caldwell, J.C., Findley, S., Caldwell, P., Santow, G., Cosford, W., et al. (Eds.). What we know about health transition: the cultural, social and behavioural determinants of health: the proceedings of an international workshop, Canberra, May 1989 (Vol. 1, pp. 92-109). Canberra: Health Transition Centre, The Australian National University.

Kunst, A., Looman, C. \& Mackenbach, J. (1991). Determinanten van binnen-jaarlijkse fluctaties in de sterfte. Tijdschr Sociale Gezondheidsz, 69, 123-131. 
Kunst, A.E., Looman, C.W.N. \& Mackenbach, J.P. (1993). Outdoor temperature and mortality in the Netherlands: a time-series analysis. American Journal of Epidemiology, 137, 331-341.

Kysely, J. (2004). Mortality and displaced mortality during heat waves in the Czech Republic. Int. J. Biometeorol, 49, 91-97.

Labonte, R. \& Torgerson, R. (2002). Frameworks for analyzing the links between globalization and health. Draft report to the World Health Organization. Saskatoon: SPHERU, University of Saskatchewan.

Ladd, B.D. \& Soskolne, C.L. (2008). A toolkit for ecoepidemiological enquiry under global ecological change. In: Soskolne, C.L., Westra, L., Kotze, L.J., Mackey, B., Rees, W.E., et al. (Eds.). Sustaining life on earth: environmental and human henlth through global governance. Lanham: Lexington Books.

Lalonde, M. (1981). A new perspective on the health of Canadians. Ottawa: Ministry of Supply and Services.

Lang, T. (1996). Food security: does it conflict with globalisation? Development, 4, 45-50.

Langford, I.H. \& Bentham, G. (1995). The potential effects of climate change on winter mortality in England and Whales. International Journal of Biometeorology, 38, 141147.

Larsen, U. (1990). The effects of monthly temperature fluctations on mortality in the United States from 1921 to 1985. Int J Biometeorology, 34, 136-145.

Larson, J.S. (1999). The conceptualization of health. Medical Care Research and Review, 56, 123-136.

Last, J. (1997). Public health and human ecology. Stamford: Appleton and Lange.

Laurian, J.U. (Ed.) (2001). Food safety in food security and food trade. Washington D.C.: International Food Policy Research Institute.

Lee, K. (1999a). The global context: a review of priority global health issues for the UK. Nuffield Trust Policy Futures for UK Health Technical Series No.1. London: Nuffield Trust.

Lee, K. (1999b). Globalization, communicable disease and equity. Development, 42(4), 3539.

Lee, K. (2001). Dialogue of the deaf? The health impact of globalisation. I of Epidem Community Health, 55, 619.

Lee, K. \& Collin, J. (2001). Review of existing empirical research on globalization and health. Geneva: World Health Organization.

Lee, K. \& Goodman, H. (2002). Global policy networks: the propagation of health care financing reform since the 1980s. In: Lee, K., Buse, K. \& Fustukian, S. (Eds.). Health policy in a globalising world. Cambridge: Cambridge University Press.

Lee, K., Fustukian, S. \& Buse, K. (2002). An introduction to global health policy. In: Lee, K., Buse, K. \& Fustukian, S. (Eds.). Health policy in a globalising world. Cambridge: Cambridge University Press.

Lee, K. (2003). Globalization and health: an introduction. New York: Palgrave Macmillan.

Lee, K., Kinh, H.V., MacKenzie, R., Gilmore, A.B., Minh, N.T., et al. (2008). Gaining access to Vietnam's cigarette market: British American Tobacco's strategy to enter 'a huge market which will become enormous' Global Public Health, 3(1), 125. 
Leemans, R. (1999). Modelling for Species and Habitats: New opportunities for problem solving. Science of the Total Environment, 240, 51-73.

Lehtonen, M. (2004). The environment-social interface of sustainable development: capabilities, social capital, institutions. Ecological Economics, 49, 199-214.

Lerner, M. (1973). Modernisation and health: a model of the health transition. Paper presented at the The annual meeting of the American public health association, San Francisco.

Lindgren, E., Tälleklint, L. \& T, P. (2000). Impact of climatic change on the Northern latitude limit and population density of the disease-transmitting European tick Ixodes ricinus. Environmental Health Perspectives, 108(2), 119-123.

Lorentz, E.N. (1963). Deterministic nonperiodic flows. Journal of Atmospheric Science, 20, 130-141.

Louria, D.B. (2000). Emerging and re-emerging infections: the social determinants. Futures, 32, 581-594.

Lubbers, R. \& Koorevaar, J. (1999). Primary globalisation, secondary globalisation, and the sustainable development paradigm: opposing forces in the 21st century. Paper presented at Expo 2000, OECD Forum for the Future: Conference on 21st century social dynamics, towards the creative society, Berlin, December 6-7.

Mackenbach, J.P., Kunst, A.E. \& Looman, C.W. (1992). Seasonal variation in mortality in The Netherlands. J Epidemiol Community Health 46, 261-265.

Mackenbach, J.P. (1993a). De achtergronden van de sterftedaling tussen 1875/79 en 1970 in Nederland. Tijdschrift Sociale Gezondheidszorg, 71(4).

Mackenbach, J.P. (1993b). De epidemiologische transitie in Nederland. Nederlands Tijdschrift voor de Geneeskunde, 3.

Mackenbach, J.P. (1994). The epidemiological transition theory. Journal of Epidemiology and Community Health, 48, 329-331.

Mackenbach, J.P. (1996). The contribution of medical care to mortality decline: McKeown revisited. Journal of Clinical Epidemiology, 11, 1207-1213.

Mackenbach, J.P. (2003). An analysis of the role of health care in reducing socioeconomic inequalities in health: the case of the Netherlands. International Journal of Health Services, 33(3), 523-541.

Macpherson, D.W. (2001). Human health, demography and population mobility. Migration and Health Newsletter, pp. 1-4.

Marks, J.S. \& McQueen, D.V. (2001). Chronic disease. In: Koop, C., Pearson, C. \& Schwartz, M. (Eds.). Critical issues in global health. San Francisco: Jossey-Bass.

Martens, P., McMichael, A.J. \& Patz, J. (2000). Globalisation, environmental change and health. Global Change and Human Health, 1(1), 4-8.

Martens, P. \& Huynen, M.M.T.E. (2001). Will global climate change reduce thermal stress in the Netherlands? Epidemiology, 12(6), 753-754.

Martens, P. (2002). Health transitions in a globalising world: towards more disease or sustained health? Futures, 37(7), 635-648.

Martens, P. \& Huynen, M.M.T.E. (2003). A future without health? Health dimension in global scenario studies. Bulletin of the World Health Organization, 81, 896 - 901.

Martens, P. (2006). Integrated Assessment models. In: Valkering, P., Amelung, B., Van der Brugge, R. \& Rotmans, R. (Eds.). More puzzle-solving for policy: Integrated Assessment from theory to practice. Maastricht: ICIS. 
Martens, P. \& Rotmans, J. (Eds.) (2002). Transitions in a globalising world. Lisse: Swets \& Zeitlinger.

Martens, W. (1998a). Health impacts of climate change and ozone depletion: an ecoepidemiologic modeling approach. Environmental Health Perspectives, 106(Suppl 1), 241-251.

Martens, W.J. (1998b). Climate change, thermal stress and mortality changes. Soc.Sci.Med, $46,331-334$.

Martin, G., Sorenson, C. \& Faunce, T. (2007). Balancing intellectual monopoly privileges and the need for essential medicines. Globalization and Health, 3, 4.

Maslow, A.H. (1954). Motivation and personality. New York: Harper and Row.

Maslow, A.H. (1968). Toward a psychology of being. New York: Van Nostrand Reinhold.

Max-Neef, M.A. (1991). Human scale development: conception, application and further reflections. New York: The Apex Press.

Mbengue, M.M. \& Thomas, U.P. (2005). The precautionary principle: torn between biodiversity, environment-related food safety and the WTO. Int. J. Global Environmental Issues, 5, 36-53.

McDaniel, P.A., Intinarelli, G. \& Malone, R. (2008). Tobacco industry issues management organizations: Creating a global corporate network to undermine public health. Globalization and Health, 4, 2.

McKeown, T.F. \& Record, R.G. (1962). Reasons for the decline of mortality in England and Whales during nineteenth century. Population Studies, 16, 94-122.

McKeown, T.F., Brown, R.G. \& Record, R.G. (1972). The modern rise of population in Europe. Population Studies, 26, 345-382.

McKeown, T.F., Record, R.G. \& Turner, R.D. (1975). An interpretation of the decline of mortality in England and Whales during the twentieth century. Population Studies, 29, 391-422.

McKeown, T.F. (1976a). The modern rise of population. London: Edward Arnold.

McKeown, T.F. (1976b). The role of medicine: dream, mirage, or nemesis? London: Nuffield Provincial Hospitals Trust.

McKeown, T.F. (1988). The origins of human disease. Oxford: Blackwell.

McLaren, L. \& Hawe, P. (2005). Ecological perspectives in health research. J. Epidemiol. Community Health, 59, 6-14.

McMichael, A.J. (1995). The health of persons, populations and planets: epidemiology comes full circle. Epidemiology, 6, 633-636.

McMichael, A.J. \& Kovats, S. (1998). Assessment of the impact on mortality in England and Whales of the heatwave and associated air pollution episode of 1976. Report to the Department of Health. London: London School of Hygiene and Tropical Medicine.

McMichael, A.J. (1999). Prisoners of the proximate: loosening the constraints on epidemiology in an age of change. Am J Epidemiol 10, 887-897.

McMichael, A.J., Smith, K.R. \& Corvalan, C.F. (2000). The sustainability transition: a new challenge. Bulletin of the World Health Organization, 78, 1067.

McMichael, A.J., Campbell-Lendrum, D., Kovats, R.S., Edwards, S., Wilkonson, P., et al. (2004). Global climate change. In: Ezzati, M., Lopez, A.D., Rodgers, A. \& Murray, C.J.L. (Eds.). Comparative quantification of health risks. Geneva: World Health Organization. 
McMichael, A.J. \& Woodruff, R. (2005). Detecting the health effects of environmental change: scientific and political challenge. Ecohealth, 2, 1-3.

McMichael, A.J. (2005). Detecting the health effects of environmental change: scientific and political challenge. Ecohealth, 2, 1-3.

McMichael, A.J., Woodruff, R. \& Hales, S. (2006). Climate change and human health: present and future risks. The Lancet, 367, 859-869.

McMichael, A.J. (2006). Population health as a primary criterion of sustainability. EcoHealth, 3, 182-186.

McMichael, A.J., Haines, A., Slooff, R. \& Kovats, R.S. (Eds.) (1996). Climate change and human health: an assessment by a task group on behalf of the World Health Organization, the World Meteorological Organization and the United Nations Environment Programme. Geneva: WHO.

Meade, B., Rosen, S., Shapouri, S., Andrews, M., Trueblood, M., et al. (2003). Food security assessment 2002-2003. Washington D.C.: United States Department of Agriculture.

Meadows, D.H., Randers, J. \& Meadows, D. (1972). The limits to growth. New York: Universe Books.

Melchior, A., Telle, K. \& Wiig, H. (2000). Globalisation and inequality - world income distribution and living standards, 1960-1998. Oslo: Royal Norwegian Ministry of Foreign Affairs.

Mercer, A. (1990). Disease, mortality, and population in transition. Leichester: Leicester University Press.

Michelozzi, P., de' Donato, F., Bisanti, L., Russo, A., Cadum, E., et al. (2005). The impact of the summer 2003 heat waves on mortality in four Italian cities. Euro Surveill, 10, 161-165.

Minesterio de Salud (2001). Análisis de la salud situación de salud en el Perú. Lima: Peru, Ministerio de Salud.

Ministerio de Salud (1996). El desafio del cambio de milenio: Un sector salud con equidad, eficiencia y calidad. Lima: Peru, Ministerio de Salud.

Ministerio de Salud (2001). Situacion de salud en el Peru: indicadores basicos 2001. Lima: Peru, Ministerio de Salud.

Ministerio de Salud (2003). Situacion de salud en el Peru: indicadores basicos 2003. Lima: Peru, Ministerio de Salud.

Miyagishima, K. \& Kaferstein, F.K. (1998). Food safety in international trade. World Health Forum, 19, 407-411.

Mooney, H.A. (Ed.) (1996). Functional roles of biodiversity. Chichester: Witey.

Mosley, W.H., Bobadilla, J.L. \& Jamison, D.T. (1992). The health transition: implications for health policy in developing countries. In: Bank, W. (Ed.). Health Sector Priorities Review. Washington D.C.

Murray, C.J.L. \& Chen, L.C. (1993). In search of a contemporary theory for understanding mortality change. Social Science and Medicine, 36, 143-155.

Murray, C.J.L. \& Lopez, A.D. (1997). Global mortality, disability, and thecContribution of risk factors: global burden of disease study. The Lancet, 349, 1436-1442.

Murray, C.J.L. \& Smith, R. (2001). Diseases of globalisation. London: Earthscan Publication Ltd. 
Murray, C.J.L. \& Lopez, A.D. (Eds.) (1996). The global burden of disease: volume 1. Geneva: World Health Organization / Harvard School of Public Health / World Bank.

Nassar, J.R. (2005). Globalization and terrorism: the migration of dreams and nightmares Lanham: Rowman \& Littlefield Publishers.

Nederveen Pieterse, J. (2004). Globalization and Culture. Lanham: Rowman \& Littlefield Publishers, Inc.

Newcomb, J. (2003). Biology and borders: SARS and the new economics of bio-security. Cambridge: Bio Economic Research Associates.

Newell, W.H. (2001). A theory of interdisciplinary studies. Issues in Integrative Studies, 19, 1-21.

NIC (2000). Global Trends 2015: A dialogue about the future with non-governmental experts. National Intelligence Council.

Ninth New Collegiate Webster's Dictionary (1977). Ottenheimer Publications Inc.

NPHP (2001). Preventing chronic disease: a strategic framework. Melbourne: National Public Health Partnership.

Nygard, K., Broch Brantseater, A. \& Mehl, R. (2005). Disseminated and chronic Lyme borreliosis in Norway, 1995-2004. EuroSurveillance 10(10), 235-238.

O'Donnell, M. (1989). The definition of health promotion. American Journal of Health Promotion, 3(3), 5.

O'Neill, M.S., Hajat, S., Zanobetti, A., Ramirez-Aguilar, M. \& Schwartz, J. (2005). Impact of control for air pollution and respiratory epidemics on the estimated associations of temperature and daily mortality. Int J Biometeorol 50(2), 121-129.

OECD (2000a). Future Ttrends 6: Aan information base for scanning the future (CD-rom).

OECD (2000b). Report on the OECD conference Environmentally Sustainable Transport: futures, strategies and best practices. Organisation for Economic Co-operation and Development. Vienna, 4-6 October 2000.

Olivera, O. \& Lewis, T. (2004). Cochabamba! Water war in Bolivia. Cambridge: South End Press.

Olshansky, S.J. \& Ault, A.B. (1986). The fourth stage of the epidemiological transition: the age of delayed degenerative diseases. Milbank Memorial Fund Quarterly, 64(3), 355-391.

Olshansky, S.J., Carnes, B.A., Rogers, R.G. \& Smith, L. (1998). Emerging infectious diseases: the fifth stage of the epidemiological transition? World Health Statistics Quarterly, 51, 207-217.

Omran, A.R. (1971). The epidemiologic transition; a theory of the epidemiology of population change. Milbank memorial fund quarterly, 49(4), 509-538.

Omran, A.R. (1983). The epidemiological transition: a preliminary update. Journal of Tropical Pediatrics, 29, 305-316.

Omran, A.R. (1998). The epidemiologic transition theory revisited thirty years later. World Health Statistics Quarterly, 51, 99-199.

PAHO (1962). Health conditions in the Americas, 1957-1960. Washington D.C.: Pan American Health Organization.

PAHO (1970). Health conditions in the Americas, 1965-1968. Washington D.C.: Pan American Health Organization.

PAHO (1974). Health conditions in the Americas, 1969-1972. Washington D.C.: Pan American Health Organization. 
PAHO (1998). Health in the Americas, 1998 edition. Washington D.C.: Pan American Health Organisation.

PAHO (2001). Health services system profile of Peru. Lima: Pan American Health Organization / World Health Organization Peru, Catholic University of Peru.

PAHO (2002). Health in the Americas, 2002 edition. Washington D.C.: Pan American Health Organisation.

Pan, W., Li, L. \& Tsai, M. (1995). Temperature Extremes and Mortality from Coronary Hearth Disease and Cerebral Infarction in Elderly Chinese. The Lancet, 345, 353355.

Pang, T., Lansang, M.A. \& Haines, A. (2002). Brain drain and health professionals: a global problem needs global soluations. . BMJ, 324, 499-500.

Parkes, M.W., Bienen, L., Breilh, J., Hsu, L., McDonald, M., et al. (2005). All hands on deck: transdisciplinary approaches to emerging infectious disease EcoHealth, 2, 258-272.

Pascual, M., Rodó, X., Ellner, S.P., Colwell, R. \& Bouma, M.J. (2000). Cholera dynamics and EI Niño-Southern Oscillation Science, 289, 1766 - 1769.

Patz, J., Campbell-Lendrum, D., Holloway, T. \& Foley, J. (2006). Impact of regional climate change on human health. Nature, 438, 310-317.

Pearce, N. (2004). The globalization of epidemiology: introductionary remarks. International Journal of epidemiology, 33, 1-5.

Pearce, N. \& Merletti, F. (2006). Complexity, simplicity, and epidemiology. International Journal of Epidemiology(35), 515-519.

Philippe, P. \& Mansi, O. (1998). Nonlinearity in the epidemiology of complex health and disease processes. Theoretical Medicine and Bioethics, 19, 591-607.

Pimm, S.L., Russell, G.J., Gittleman, J.L. \& Brooks, T.M. (1995). The future of biodiversity. Science, 269, 1466-1474.

Pirard, P., Vandentorren, S., Pascal, M., Laaidi, K., Le Tertre, A., et al. (2005). Summary of the mortality impact assessment of the 2003 heat wave in France. Euro Surveill 10, 153-156.

Pongsiri, M.J. \& Roman, J. (2007). Examining the links between biodiversity and human health: an interdisciplinary research Initiative at the U.S. Environmental Protection Agency EcoHealth, 4, 82-85.

Powles, J. (1992). Changes in diseases patterns and related social trends. Social Science and Medicine, 35, 377-387.

Prigogine, I. \& Stenger (1984). Order out of chaos: man's new dialogue with nature. Toronto: Bantam Books

Prigogine, I. (1997). The end of certainty. New York: The Free Press.

Pritchett, L. \& Summers, L. (1996). Wealthier is healthier. I Hum Resources, 31, 841-868.

Ramsey, F.L. \& Schafer, D.W. (1997). The statistical sleuth: a course in methods of data analysis. Belmont: Duxbury Press.

Rapport, D.J., Constanza, R. \& McMichael, A.J. (1998). Assessing ecosystem health. TREE, 13, 397-402.

Ravetz, J. (1999). What is Post-Normal Science. Futures, 31, 647-653.

Razzell, P.E. (1974). An interpretation of the modern rise of population in Europe: a critique. Population Studies, 28, 5-12. 
Reeves, P.M. (2000). Coping in cyberspace: the impact of Internet use on the ability of HIV-positive individuals to deal with their illness. J Health Commun., 5 (Suppl), 47-59.

Reid, C. (2004). Wounds of exclusion; poverty, women's health and social justices. Edmonton: Qualitative Institute Press.

Reinicke, W.H. (1998). Global public policy: governing without government? Washington D.C.: Brookings Institution Press.

Reiser, O.L. \& Davies, B. (1994). Planetary democracy: an introduction to scientific humanism and applied semantics. New York: Creative Age Press.

Reitsma, J.B. \& Dalstra, J.A.A. (1999). Cardiovascular disease in the Neterlands, 1975 to 1995, decline in mortality, but increasing numbers of patients with chronic conditions. Heart, 82, 52-56.

Rennen, W. \& Martens, P. (2003). The globalisation timeline. Integrated Assessment, 4, 137144.

Reyes, J. \& Ochoa, L.H. (2001). Encuesta Demográfica y de Salud-ENDES 2000. Lima: Instituto Nacional de Estadistica e Informatica.

Ritzer, G. (1993). The McDonaldization of society. Thousand Oaks: Pine Forge Press.

Ritzer, G. (1998). The McDonaldization thesis. London: Sage.

RIVM (2006). Ziekte van Lyme neemt toe. Persbericht 27 April 2006. http://www.rivm.nl/persberichten/2006/toenameziektevanlyme.jsp. Retrieved May 25, 2006.

Robinson, J. (2004). Squaring the circle? Some thoughts on the idea of sustainable development. Ecological Economics, 48, 369-384.

Rogers, G.R. \& Hackenberg, R. (1987). Extending epidemiological transition theory: a new stage. Social Biology, 34(3-4), 234-243.

Rooney, C., McMichael, A.J., Kovats, R.S. \& Coleman, M.P. (1998). Excess mortality in England and Whales, and in Greater London, during the 1995 heatwave. Journal of Epidemiology and Community Health, 53, 482-486.

Rosenau, J.N. (1995). Governance in the twenty-first century. Global Governance, 1, 13-43.

Rosenfield, P. (1992). The potential of transdisciplinary research for sustaining and extending linkages between the health and social sciences. Social Science and Medicine, 35(11), 1343-1357.

Rossi, G., Vigotti, M.A., Zanobetti, A., Repetto, F., Gianelle, V., et al. (1999). Air Pollution and Cause-Specific Mortality in Milan, Italy, 1980-1989. Archives of Environmental Health, 54(3), 158-164.

Rothman, D. (2006). Scenarios: structured thinking about the future. In: Valkering, P., Amelung, B., Van der Brugge, R. \& Rotmans, J. (Eds.). More puzzle-solving for policy: Integrated Assessment from theory to practice. Maastricht: ICIS.

Rotmans, J. \& Dowlatabadi, H. (1998). Integrated assessment modeling. In: Raynes, S. \& Malone, E. (Eds.). The tools for policy analysis, . Columbus, Ohio: Battelle Press.

Rotmans, J. (1998). Methods for IA: the challenges and opportunities ahead. Environmental Modeling and Assessment, 3, 155-179.

Rotmans, J. (1999). Integrated Assessment: a bird's-eye view. Maastricht: ICIS.

Rotmans, J. \& de Vries, H.J.M. (Eds.) (1997). Perspectives on global change: the TARGETS approach. Cambridge: Cambridge University Press. 
Ruwaard, D. \& Kramers, P.G.N. (Eds.) (1993). Volksgezondheid Toekomst Verkenning: de gezondheidstoestand van de Nederlandse bevolking in de periode 1950-2010. Bilthoven: RIVM.

Ruwaard, D. \& Kramers, P.G.N. (Eds.) (1997). Volksgezondheid Toekomst Verkenning 1997: de som der delen. Bilthoven: RIVM.

Saez, M., Sunyer, J., Castellsague, J., Murillo, C. \& Anto, J.M. (1995). Relationship between weather temperature and mortality: a time-series analysis approach in Barcelona. International Journal of Epidemiology, 24, 576-582.

Sala, O.E., Chapin, F.S., Armesto, J.J., Berlow, E., Bloomfield, J., et al. (2000). Biodiversity: global biodiversity scenarios for the year 2100. Science, 287, 1770-1774.

Sander, H. (1996). Multilaterism, regionalism, and globalisation: the challenges to the world trading system. In: Sander, H. \& Anotai, A. (Eds.). World trade after the Uruguay round: prospects and policy options for the twenty-first century. London: Routledge.

Sartor, F., Snacken, R., Demuth, C. \& Walckiers, D. (1995). Temperature, ambient ozone levels, and mortality during summer 1994, in Belgium. Environ Res., 70(2), 105113.

Scally, G. (1998). Public Health. British Medical Journal, 317, 584-586.

Schaanning, J., Finsen, H. \& Lereim, I. (1986). Effects of cold air inhalation combined with prolonged sub-maximal exercise on airway function in healthy young males. European Journal of Respiratory Diseases, suppl. 143, 74-77.

Schiller, H.I. (1991). Not yet the pos-imperialist era. Critical Studies in Mass Communication, 8, 13-28.

Scholte, J.A. (2000). Globalization: a critical introduction. New York: Palgrave.

Schuerkens, U. (2003). The social and anthropological study of globalization and localization. Current Sociology, 51, 209-222.

Schulze, E.D. \& Mooney, H.A. (Eds.) (1994). Biodiversity and ecosystem function. Berlin: Springer.

Schwartz, M.W., Brigham, C.A., Hoeksema, J.D., Lyons, K.G., Mills, M.H., et al. (2000). Linking Biodiversity to Ecosystem Function: Implications for conservation ecology. Oecologica, 122, 297-305.

Secretariat of the Convention on Biological Diversity (2006). Global Biodiversity Outlook 2. Montreal: Secretariat of the Convention on Biological Diversity.

Shiva, V. (2004). The future of food: countering globalization and recolonisation of Indian agriculture. Futures, 36, 715-732.

Siesling, S., van Dijk, J.A.A.M., Visser, O. \& Coebergh, J.W.W. (2003). Trends in incidence of and mortality from cancer in The Netherlands in the period 1989-1998. European Journal of Cancer, 39, 2521-2530

Sieswerda, L.E., Soskolne, C.L., Newman, S.C., Schopflocher, D. \& Smoyer, K.E. (2001). Toward measuring the impact of ecological disintegrity on human health. Epidemiology, 12, 28-32.

Singer, P. (2002). One world. New Haven: Yale University Press.

Smaller, C. (2005). Planting the rights seed: a human rights perspective on agricultural trade and the WTO. Geneva: 3D Publications \& Institute for Agriculture and Trade Policy (IATP). 
Soberon, J., Rodriquez, P. \& Vasquez-Domingues, E. (2000). Implications of the hierarchical structure of biodiversity for the development of ecological indicators of sustainable use. Ambio, 29, 136-142.

Soskolne, C. \& Broemling, N. (2002). Eco-epidemiology: on the need to measure health effects from global change. Global Change \& Human Health, 3(1), 58-66.

Soskolne, C. \& Bertollini, R. (2002). Global ecological integrity, global change and public health. In: Aguirre, A., Ostfeld, R., Tabor, G., House, C. \& Pearl, M. (Eds.). Conservation medicine: ecological health in practice (pp. 372-382). New York: Oxford University Press.

Soskolne, C. (2003). Measuring the impact of ecological disintegrity on human health: a role for epidemiology. In: Rapport, D., Lasley, W., Rolston, D., Nielsen, N., Qualset, C., et al. (Eds.). Managing For Healthy Ecosystems (pp. 259-265). Boca Raton: Lewis Publishers.

Soskolne, C., Butler, C., IJsselmuiden, C., London, L. \& von Schirnding, Y. (2007). Toward a global agenda for research in environmental epidemiology. Epidemiology and Society, 18(1), 162-166.

Sreenivasan, G. \& Benatar, S. (2006). Challenges for global health in the 21st century: some upstream considerations. Theoretical Medicine and Bioethics, 27, 3-11.

Statistics Netherlands (2004). De Nederlandse samenleving2004: sociale trends. Voorburg: Statistics Netherlands,.

Statistics Netherlands (2006a). Statline Historische reeksen: historie bevolking. http://statline.cbs.nl/. Retrieved July, 10, 2006.

Statistics Netherlands (2006b). Statline Historische reeksen: historie gezondheid. http://statline.cbs.nl/ Retrieved July, 10, 2006.

Statistics Netherlands (2006c). Statline Historische reeksen: historische overlevingstafels. http://statline.cbs.nl/. Retrieved July, 10, 2006.

Statistics Netherlands (2006d). Statline Mens en Maatschappij: gezondheid en welzijn. http://statline.cbs.nl/. Retrieved July, 10, 2006.

Stevens, C. (2003). Food trade and food policy in Sub-Saharan Africa: old myths and new challenges. Development Policy Review, 21, 669-681.

Stivoro (2002). Roken, de harde feiten. Den Haag: Stivoro Rookvrij.

Stott, P.A., Stone, D.A. \& Allen, M.R. (2004). Human contribution to the European heatwave of 2003. Nature, 432, 610-614.

Susser, M. \& Susser, E. (1996). Choosing a future for epidemiology: II. From black box to Chinese boxes and eco-epidemiology. Am J Public Health, 86, 674-677.

Swart, R.J., Raskin, P. \& Robinson, J. (2004). The problem of the future: sustainability science and scenario analysis. Global Environmental Change, 14, 137-146.

Szretzer, S. (1988). The importance of social intervention in Britain's mortality decline 1850-1914: a re-interpretation of the role of public health. Social History of Medicine, 1, 5-37.

Terris, M. (1983). The complex tasks of the second epidemiologic revolution. Journal of Public Health Policy, 4(1), 8-24.

Terris, M. (1985). The changing relationships of epidemiology and society. Journal of Public Health Policy, 6(15-36).

Timen, A. (2003). SARS en public health in Nederland. Infectieziekten Bulletin, 14. 
UN (2000). United Nations Millennium Declaration. General Assembly resolution 55/2 of 8 September 2000

UN (2003a). Trends in total migrant stock: the 2003 revision. New York: United Nations, Department of Economic and Social Affairs, Population Division.

UN (2003b). World population prospects: the 2002 revision. New York: Population Division of the Department of Economic and Social Affairs of the United Nations Secretariat.

UN (2004). World economic and social survey 2004: international migration. New York: United Nations, Department of Economic and Social Affairs.

UN (2005). UN Millennium Development Gonls. http:/www.un.org/millenniumgoals/. Retrieved September, 2, 2007.

UN (2007a). The Millennium Development Gonls report 2007. New York: United Nations.

UN (2007b). World population prospects: the 2006 revision population database. http://esa.un.org/unpp/ Retrieved September 16, 2007

UNDP (1997). Reconceptualizing governance. A UNDP discussion paper.New York: United Nations Development Programme.

UNDP (1999). Human development report 1999: globalization with a human face. New York: Oxford University Press.

UNDP (2005). Human development report 2005: international cooperation at a crossroads- aid, trade and security in an unequal world. New York: United Nations development Programme.

UNEP (1992). Convention on Biological Diversity.

UNEP (1995). Global biodiversity assessment. Cambridge: Cambridge University Press.

UNEP (2002). Global Environmental Outlook 3. London: Earthscan.

UNEP \& Global Environment Facility (2007). International initiative gets underway to track progress in conserving biodiversity: Press release July 12, 2007.

Urry, J. (2003). Global complexity. Cambridge: Polity Press.

Urry, J. (2005a). The complexities of the global. Theory, Culture \& Society, 22, 255-267.

Urry, J. (2005b). The complexity turn. Theory, Culture \& Society 22, 1-14.

Valkering, P., Amelung, B., van Brugge, R. \& Rotmans, J. (Eds.) (2006). More puzzlesolving for policy: integrated assessment from theory to practice. Maastricht: ICIS.

van Asselt, M.B.A. (2000). Perspectives on uncertainty and risk: the PRIMA approach for decision support. Dordrecht: Kluwer.

van Asselt, M.B.A., Rotmans, J. \& Greeuw, S.C.H. (2001). Puzzle-solving for policy: a provisional handbook for Integrated Assessment. Maastricht: ICIS.

van Asselt, M.B.A. \& Rijkens-Klomp, N. (2002). A look in the mirror: reflection on participation in Integrated Assessment from a methodological perspective. Global Environmental Change, 12, 167-184.

van Asselt, M.B.A., Rotmans, J. \& Rothman, D.S. (2005). Scenario innovation: experiences from a European experimental garden. Oxford: Taylor \& Francis.

van Asselt, M.B.A. (2006). The challenge of uncertainty and plurality in Integrated Assessment. In: Valkering, P., Amelung, B., Van der Brugge, R. \& Rotmans, J. (Eds.). More puzzle-solving for policy: Integrated Assessment from theory to practice. Maastricht: ICIS.

van Breukelen, G. (1995). MGK Statistiek: lineaire en binaire regressie analyse. Maastricht: Maastricht University. 
van de Laar, W.J.M. \& op de Coula, E.L.M. (2004). HIV and sexually transmitted infections in the Netherlands in 2003. Report No. 441100020/2004. Bilthoven: Netherlands Institute of Public Health and the Environment.

van den Hurk, B., Klein Tank, A., Lenderink, G., van Ulden, A., van Oldenborgh, G.J., et al. (2006). KNMI Climate Change Scenarios 2006 for the Netherlands. De Bilt: The Royal Meteorological Institute for the Netherlands (KNMI)

van der Eerden, L.J.M., Bosman, A. \& Visser, L.G. (2002). Malaria uit West Africa neemt toe Infectieziekten Bulletin, 13, 398-405.

van der Meulen, A. (2005). Sterfte aan hart- en vaatziekten sinds 1970 gehalveerd. Centraal Bureau voor de Statistiek Webmagazine http://www.cbs.nl/nl$\mathrm{nl} / \mathrm{menu} /$ themas/mens-maatschappij/bevolking/publicaties/artikelen/2005-1785wm.htn Retrieved July 3, 2006.

van der Poel, W.H.M., van der Heide, R., Bakker, D., de Loof, M., de Jong, J., et al. (2005). Attempt to detect evidence for tick-borne Encephalitis virus in ticks and mammalian wildlife in the Netherlands. Vector-borne and Zoonotic Diseases, 5, 5864.

van der Wilk, E.A., Achterberg, P.W. \& Kramers, P.G.N. (2001). Lang leve Nederland! Bilthoven: RIVM.

van Doesburg, M.J. (1995). A survey of summer smog in the Netherlands in 1994 Bilthoven: RIVM.

van Leest, L.A.T.M., Koek, H.L., Bots, M.L. \& Verschuren, W.M.M. (2002). Hart- en vaatziekten in Nederland 2002. Den Haag: Nederlandse Hartstichting.

van Notten, P.W.F., Rotmans, J., van Asselt, M.B.A. \& Rothman, D.S. (2003). An updated scenario typology. Futures, 35, 423-443.

van Oers, J.A.M. (2002). Volksgezondheid toekomst verkenning 2002. Bilthoven: RIVM.

van Soest, J. (Ed.) (1998). Biodiversity (in Dutch). Utrecht: KNNV Uitgeverij.

VanLeeuwen, J.A., Waltner-Toews, D., Abernathy, T. \& Smitt, B. (1999). Evolving models of human health toward an ecosystem context. Ecosystem Health, 5(3), 204-219.

Wackernagel, M. \& Rees, W. (1996). Our ecological footprint: reducing human impact on the earth. Philadelphia: New Society Publishers.

Waldrop, M. (1992). Complexity: the emerging science and the edge of order and chaos. London: Simo and Schuster.

Walt, G. (2000). Globalization and health. Paper presented at the Medact Meeting.

WBCSD (1998). Exploring sustainable development: global scenarios 2000-2050. London: World Business Council for Sustainable Development.

Weyant, J., Davidson, O., Dowlatabadi, H., Edmonds, J., Grubb, M., et al. (1996). Integrated assessment of climate change: An overview and comparison of approaches and results. In: Bruce, J., Lee, H. \& Haites, E. (Eds.). Climate change 1995: economic and social Dimensions. Contribution of Working Group III to the Second Assessment Report of the Intergovernmental Panel on Climate Change. Cambridge: Cambridge University Press.

WHO (1946). World Health Organization constitution. Geneva: World Health Organization.

WHO (1986). Ottawa Charter for Health Promotion. First International Conference on Health Promotion, 21 November 1986. Ottawa, Canada.

WHO (1999). The world health report 1999. Geneva: World Health Organization.

WHO (2000). The world health report 2000. Geneva: World Health Organization. 
WHO (2001a). Macroeconomics and health: investing in health for economic development. Geneva: World Health Organization, Commission on Macroeconomics and Health.

WHO (2001b). The world health report 2001. Geneva: World Health Organization.

WHO (2002a). The European health report 2002. Copenhagen: World Health Organization, Regional Office for Europe.

WHO (2002b). The world health report 2002: reducing risks, promoting healthy life. Geneva: World Health Organization.

WHO (2002c). World report on violence and health. Geneva: The World Health Organization.

WHO \& WTO (2002). WTO agreements and public health. Geneva: World Health Organization and the World Bank.

WHO (2003a). Disease outbreak news: update 95-Sars, chronology of a serial killer. http://www.who.int/csr/don/2003_07_04/en/. Retrieved August 7, 2007.

WHO (2003b). Severe acute respiratory syndrome (SARS): status of the outbreak and lessons for the immediate future. Geneva: WHO.

WHO (2003c). World Health Organization Framework Convention on Tobacco Control (WHO FCTC). For the full text, see http://www.who.int/tobacco/framework/en/.

WHO (2004). World health report 2004: changing history. Geneva: World Health Organization.

WHO (2006). Pandemic flu - communicating the risks: the Bulletin interview with Dr Margaret Chan Bulletin of the World Health Organization, 84, 9-11.

WHO (2007). EuroHEAT: Improving public health responses to weather extremes, in particular to heat-waves. http://www.euro.who.int/globalchange/Topics/20050524_2 Retrieved September, 6, 2007.

Wilcox, B. \& Colwell, R. (2005). Emerging and reemerging infectious diseases: biocomplexity as an interdisciplinary paradigm. Ecohealth, 2, 244-257.

Wilkinson, P., Pattenden, S., Armstrong, B., Fletcher, A., Kovats, S., et al. (2004). Vulnerability to winter mortality in elderly people in Britain: population based study. BMJ, 329, 647 .

Williams, C.C. \& Millington, A.C. (2004). The diverse and contested meanings of sustainable development. The Geographical Journal, 170, 99-104.

Wilson, M.E. (1995). Travel and the emergence of infectious diseases. Emerging infectious diseases, 1, 39-46.

Wolleswinkel-van den Bosch, J. (1998). The epidemiological transition in The Netherlands. Erasmus University Rotterdam, Rotterdam.

Wolleswinkel - Van den Bosch, J., Looman, C.W.N., van Poppel, F.W.A. \& Mackenbach, J.P. (1997). Cause-specific mortality trends in The Netherlands, 1875-1992: a formal analysis of the epidemiologic transition. International Journal of Epidemiology 26, 772-781.

Wolleswinkel - Van den Bosch, J., van Poppel, F.W.A., Tabeau, E. \& Mackenbach, J.P. (1998). Mortality decline in The Netherlands in the period 1850-1992: a turning point analysis. Social Science and Medicine, 47, 429-444

Woods, R. \& Shelton, N. (1997). An atlas of Victorian mortality. Liverpool: Liverpool University Press. 
Woods, R. (2000). The demography of Victorian England and Wales. Cambridge: Cambridge University Press.

Woodward, D., Drager, N., Beaglehole, R. \& Lipson, D. (2001). Globalization and health: a framework for analysis and action. Bulletin of the World Health Organisation, 79(9), 875-881.

World Bank (1999). World development indicators. Washington D.C.: World Bank.

WRI (1996). World Resources 1996-1997: the urban environment. Washington D.C.: World Resources Institute.

WRI (2000). World Resources 2000-2001: people and ecosystems, the fraying of the web. Washington, D.C.: World Resources Institute.

WTO (2000). European Communities measures affecting asbestos and asbestos-containing products. WT/DS135R. Panel Report. Geneva: World Trade Organization.

WTO (2001). European Communities measures affecting asbestos and asbestos-containing products. WT/DS135/AB/R. Appellate Body Report. Geneva: World Trade Organization.

WTO (2003). The World Trade Organization in brief. Geneva: World Trade Organization.

WTO (2005). World trade report 2005: exploring the links between trade, standards and the WTO. Geneva: World Trade Organization.

Yassi, A., Kjellstrom, T., Kok de, T. \& Guidotti, T.L. (2001). Basic environmental health. New York: Oxford University Press.

Zwi, A., Fustukian, S. \& Sethi, D. (2002). Globalisation, conflict and the humanitarian response. In: Lee, K., Buse, K. \& Fustukian, S. (Eds.). Health policy in a globalising world. Cambridge: Cambridge University Press. 


\section{SUMMARY}

In light of the international commitment to sustainable development, exploring our future has become a matter of urgency and relevance. Studying the future of our health should be a vital part of this. In doing so, we need to recognise that the prospects for our health increasingly depend on the various processes of globalisation. In 2003, for example, the world held its breath during a historically unprecedented situation: the rapid spread of the Severe Acute Respiratory Syndrome (SARS) caused by worldwide human travelling. However, this is only one of the many possible health implications of globalisation. Global health research addresses the ways in which globalisation is impacting on both health determinants and outcomes. An important objective of this Ph.D. Thesis is to provide a better understanding of the possible (future) health implications of the globalisation process, including global environmental change. The central question underlying this study is as follows: How will future health evolve given anticipated globalisation trends?

Chapter 1 states that our (future) health should be an integral part of the current discussions about sustainable development and sustainable globalisation. Population health is perceived as the integrated outcome of the economic, social-cultural, institutional, and ecological determinants that affect a population's physical, mental, and social abilities to function normally. Globalisation is defined as a process characterised by a growing intensity, extensity, and velocity of institutional, economic, social-cultural and ecological interactions, resulting in transborder processes and effects. In order to address the main question, the following research objectives are identified:

1. To assess past developments in population health, both in the developed and developing world.

2. To develop an integrated framework to analyse the consequences of globalisation for population health, accounting for plurality along the way.

3. To explore future health given anticipated globalisation pathways and associated developments in important health determinants, both in the developed and developing world.

4. To explore the (future) health impacts of global environmental change, focussing on global climate change and global biodiversity loss.

Chapter 2 describes our methodological framework, which is based on a systems-approach towards both globalisation and population health. It is discussed that the complexity involved in assessing the health impacts of globalisation is characterised by holism and systems thinking, uncertainty and plurality, and inter- and transdisciplinary. The increasing awareness that a system-based approach towards (global) population health is necessary, is in line with the more general 'complexity turn' in science. In view of the main 
research question, a system-based approach implies a lower emphasis on prediction, but an accompanying greater emphasis on understanding of the processes involved, acknowledging inherent uncertainties, and exploring alternative health futures. Integrated Assessment (IA) provides such an approach, and associated tools.

Chapter 3 presents an overview of the past developments in health, using the framework of the epidemiological transition theory. A descriptive analysis of the changes observed in the Netherlands and in Peru provides insights into the different transition experiences of these two countries. The Dutch epidemiological changes clearly demonstrate that the Netherlands currently finds itself in the fourth stage of the Western transition model. The changes in the Peruvian health situation are a good example of the third stage of the nonWestern transition model. A complicating factor for Peru is the epidemiological polarisation within the country. Subsequently, we assess what we can learn from the epidemiological transition in order to improve our understanding of the broader context of population health. The original theory was based on a rather deterministic view of population health. However, not all countries follow the same transition pathway, and we should take into account that different (groups of) countries will differ in their future transition experience. The epidemiological transition also shows that the system can respond in an unpredictable way; this implies that multiple health futures are possible. Additionally, the causal mechanism behind the observed epidemiological changes cannot be explained using reductionist approaches; not one or two main factors triggered the vast improvements in our health, but a combination of reinforcing developments. This is in line with our claim that a more integrated view of health determination-accounting for the broader context of population health-is fundamental to advance our understanding.

This multicausality of population health plays an important role in Chapter 4, which describes the stepwise development of a new conceptual framework for the health implications of the globalisation process. Especially in the phase of issue- or problem-framing, the development of a conceptual model improves the integrated understanding of all key components and processes involved. A review of existing health models forms the basis for the identification and structuring of the most important factors influencing health. The nature of the identified health determinants (institutional, economic, social-cultural, and ecological) and their level of causality (proximal, distal, and contextual) are combined into a basic framework that conceptualises the complex multicausality of population health. Accordingly, we distinguish the following important features of the globalisation process: new global governance structures, global markets, global communication and diffusion of information, global mobility, cross-cultural interaction, and global environmental changes. 
These features all operate at the contextual level of health determination, influencing the distal factors identified within our framework, andsubsequently- the proximal health determinants. The literature review underlying this study has been very extensive and the resulting framework reflects the diversity of views in various relevant disciplines. Our conceptual framework is rooted in an integrated approach towards both population health and globalisation, and provides valuable insights in how to organise the various factors involved in studying global health.

Due to the complex interplay between the various factors within the global system, multiple future pathways are possible. Chapter 5 explores future developments in population health resulting from different globalisation pathways, for both the developed and developing countries. Based on nine selected global scenario studies (with a total of 35 scenarios), we conclude that the health dimension is largely missing in past global scenario exercises. Globalisation, on the other hand, is included as one of the most important drivers of future change. Among the selected scenarios, the following set of globalisation pathways can be identified: a globalising world with a marketoriented economic focus, a globalising world with a 'sustainability' focus, and a fragmented world. For each group two main variants can be identified. We link the institutional, economic, social-cultural, and environmental developments described by three existing scenario studies (SRES, GEO3, MA) to a set of possible health futures ('age of emerging infectious diseases', 'age of medical technology', 'age of sustained health', and 'age of chronic diseases for nonwestern countries'.) For the MA-scenarios, we use the conceptual model developed earlier to structure this exercise. This analysis provides an integrated outlook on fundamentally different health futures; it provides useful insights into how to deal with health in scenarios and shows that a comprehensive picture of future health evolves when all relevant institutional, economic, social-cultural, and environmental developments are taken into account.

The next two Chapters focus on the health impacts of global environmental change. Chapter 6 investigates the climate change-induced changes in temperature-related mortality in the Netherlands. Although global climate change is likely to be accompanied by an increase in summer temperatures, winters are expected to be milder as well. For many temperate or cold regions (including the Netherlands) it is, therefore, often stated that the decrease in cold-related mortality could counterbalance the increasing rates of heat-related mortality, even resulting in a reduction in mortality. In this study, the relationship between mortality and (extreme) temperature is assessed using an epidemiological approach (time-series analysis and episode analysis). Based on the resulting quantitative exposure-effect relationships, we assess the changes in the proportion temperature-related deaths (total mortality, cardiovascular 
mortality, respiratory mortality, and mortality in the $65+$ age group) attributable to climate changes, using recent estimates of daily temperatures for 2050 (KNMI'06 scenarios). Our results show that in the two scenarios without a change in air circulation patterns ( $G$ and $W$ ), temperature-related mortality indeed declines; when we take into account that people can adjust to the new climate through acclimatisation, however, temperature-related mortality virtually remains unchanged. The change in air circulation patterns projected in the other two scenarios ( $\mathrm{G}+$ and $\mathrm{W}+$ ) yield different outcomes; temperature related mortality still decreases without acclimatisation, but increases with acclimatisation.

Chapter 7 quantitatively assesses the association between biodiversity loss and health by means of a regression analysis using country level indicators for biodiversity loss, population health, and socio-economic development. Global biodiversity loss could result in compromised ecosystem functions, which, in turn, could negatively influence human health. Therefore, we expected that the relationship between the biodiversity loss and human health to be negative. However, this study was not able to provide any statistical proof of the expected negative association between loss of biodiversity and human health. There may be several reasons for our findings: lack of suitable indicators, possible non-randomness in the selection of countries, limitations of regression analysis, possible non-linearity of the relationship, difficulties regarding the role of socio-economic development (e.g., separation of consumption from consequence), and inappropriate scale level. Hence, the challenges encountered and the need for new innovative methodologies are perfectly illustrated by this study.

Chapter 8 recapitulates the main conclusions drawn in this Ph.D. Thesis, provides a brief methodological reflection, and offers an outlook to future work. The main research question underlying this study asks how future health will evolve given anticipated globalisation trends. However, the answer to this question cannot be captured in the prediction of a single picture of future health. As a result, the focus of this Ph.D. Thesis is aimed at enhancing our understanding of the processes involved in order to better anticipate future risks as well as the opportunities provided to us by the globalisation process. The methodological approach underlying this study is meant to contribute to the ongoing discussions and development of methods in the analysis of (future) health in a globalising world. It is discussed, in retrospect, how we addressed the methodological challenges (i.e., holism and systems thinking, uncertainty and plurality, inter- and transdisciplinarity) within our research. One important avenue for future research is to further develop integrated health scenarios, using a combination of suitable tools. With regard to the assessment of the health impacts of global environmental change, we underline the need for 
innovative system-based research approaches. Finally, we briefly point out that the international policy agenda also needs to reflect the reality that contemporary globalisation is an important health determinant. 


\section{SAMENVATTING}

In het licht van de toegenomen internationale aandacht voor duurzame ontwikkeling, is het verkennen van onze toekomst een urgente en relevante kwestie geworden. Onderzoek naar onze toekomstige gezondheid zou hier een essentieel onderdeel van moeten uitmaken. Hierbij moeten we onderkennen dat de vooruitzichten voor onze gezondheid in toenemende mate worden beïnvloed door het mondialiseringsproces. In 2003, bijvoorbeeld, hield de hele wereld zijn adem in gedurende een historisch ongekend verschijnsel: de razendsnelle verspreiding van SARS (Severe Acute Respiratory Syndrom) via het vliegverkeer. Dit is echter slechts één van de vele mogelijke gezondheidseffecten van mondialisering. 'Global Health'-onderzoek bestudeert de verschillende effecten van mondialisering op gezondheidsdeterminanten en -uitkomsten. Een belangrijke doelstelling van dit promotieonderzoek is het verkrijgen van een beter inzicht in de mogelijke (toekomstige) gezondheidseffecten van mondialisering, inclusief mondiale milieuveranderingen. De centrale vraag die aan deze studie ten grondslag ligt luidt als volgt: Hoe zal gezondheid zich in de toekomst ontwikkelen als gevolg van mogelijke mondialiseringstrends?

Hoofdstuk 1 stelt dat onze (toekomstige) gezondheid een integraal onderdeel zou moeten vormen van de huidige discussies over duurzame ontwikkeling en duurzame mondialisering. Gezondheid wordt in deze studie gezien als de integrale uitkomst van economische, sociaal-culturele, institutionele en ecologische determinanten welke de fysieke, mentale en sociale capaciteiten van een bevolking om normaal te functioneren beïnvloeden. Mondialisering wordt gekenmerkt door een toenemende intensiteit, extensiteit en snelheid van institutionele, economische, sociaal-culturele en ecologische interacties, waardoor er grensoverstijgende (transnationale) processen en effecten ontstaan. Rondom de centrale onderzoeksvraag, zijn de volgende onderzoekdoelstellingen geïdentificeerd:

1. Het analyseren van de historische ontwikkelingen in gezondheid, zowel in de ontwikkelde als ontwikkelingslanden.

2. Het ontwikkelen van een geïntegreerd raamwerk voor de analyse van de gezondheidseffecten van mondialisering, welke rekening houdt met de diversiteit aan verschillende perspectieven.

3. Het verkennen van toekomstige gezondheid, gegeven te verwachten mondialiseringstrends en geassocieerde ontwikkelingen in gezondheidsdeterminanten, voor zowel de ontwikkelende als de ontwikkelingslanden.

4. Het verkennen van de (toekomstige) gezondheidseffecten van mondiale milieuveranderingen, met een focus op klimaatverandering en verlies aan biodiversiteit. 
Hoofdstuk 2 beschrijft het methodologische raamwerk van ons onderzoek, welke is gebaseerd op een systeembenadering van zowel mondialisering als gezondheid. De inherente complexiteit van onderzoek naar de gezondheidseffecten van mondialisering wordt gekenmerkt door holisme en systeemdenken, onzekerheid en pluraliteit, en inter- en transdisciplinariteit. De toenemende bewustwording dat een systeembenadering van mondiale gezondheid noodzakelijk is, komt overeen met de meer algemene 'complexity turn' in de wetenschap. Een systeembenadering impliceert een verschuiving van 'voorspellen' naar meer aandacht voor de processen binnen het systeem, het erkennen van onzekerheden, en het verkennen van meerdere mogelijke toekomstontwikkelingen in gezondheid. Integrated Assessment (IA) wordt gekenmerkt door een dergelijke geïntegreerde benadering, en bijbehorende methoden.

Hoofdstuk 3 geeft een overzicht van de historische ontwikkelingen in onze gezondheid, gebruikmakend van de epidemiologische transitie theorie. Een analyse van de waargenomen veranderingen in Nederland en Peru geeft inzicht in de verschillende transitie-ervaringen in deze twee landen. De Nederlandse gezondheidsontwikkelingen tonen duidelijk aan dat Nederland zich momenteel in de vierde fase van het westerse transitiemodel bevindt. De veranderingen in de Peruaanse gezondheidssituatie zijn een goed voorbeeld van de derde fase van het niet-westerse transitiemodel. Een complicerende factor voor Peru is de epidemiologische polarisatie in het land. Vervolgens wordt er kort beschreven wat we vanuit een systeemperspectief van de epidemiologische transitie kunnen leren om een beter begrip te krijgen van de bredere context van gezondheid. De oorspronkelijke transitietheorie was gebaseerd op een deterministische kijk op gezondheid. Echter, niet alle landen volgen dezelfde transitieweg en we moeten er dus rekening mee houden dat verschillende (groepen van) landen ook kunnen verschillen in hun toekomstige transitiepad. De epidemiologische transitietheorie toont bovendien aan dat het systeem zich op een onvoorspelbare wijze kan ontwikkelen; dit impliceert dat meerdere toekomstontwikkelingen in gezondheid mogelijk zijn. Daarnaast, kan het causale mechanisme achter de waargenomen epidemiologische veranderingen niet worden verklaard op een reductionistische wijze; niet één of twee belangrijke factoren brachten de verbeteringen in onze gezondheid teweeg, maar de combinatie van meerdere en elkaar versterkende ontwikkelingen. Dit is in overeenstemming met onze eerdere stelling dat een geïntegreerde benadering van gezondheid- die rekening houdt met de bredere context waarin gezondheid zich ontwikkeld- fundamenteel is voor een beter begrip van 'global health'. 
De multi-causaliteit van gezondheid speelt ook een belangrijke rol in hoofdstuk 4, waarin de stapsgewijze ontwikkeling van een conceptuele raamwerk voor de gezondheidseffecten van mondialisering wordt beschreven. De ontwikkeling van een geïntegreerd model draagt bij aan een beter inzicht in alle relevante componenten en processen in kwestie. Een review van bestaande gezondheidsmodellen vormt de basis voor de identificatie en structurering van de belangrijkste factoren die onze gezondheid beïnvloeden. De aard van de geïdentificeerde gezondheidsdeterminanten (institutioneel, economisch, sociaal-cultureel, en milieu) en hun plek in de causale keten (proximaal, distaal, en contextueel) worden gecombineerd tot een basiskader, welke de complexe multi-causaliteit van gezondheid weergeeft. Vervolgens worden de volgende aspecten van mondialisering onderscheiden: mondiale beleidsstructuren, mondiale markten, mondiale communicatie en de verspreiding van informatie, crossculturele interactie, mondiale mobiliteit, en mondiale milieuproblemen. Deze aspecten opereren op het contextuele niveau van gezondheidsdeterminatie, beïnvloeden de distale factoren in het raamwerk en, vervolgens, de proximale gezondheidsdeterminanten. Het literatuuronderzoek dat ten grondslag ligt aan deze studie is zeer divers en het resulterende raamwerk reflecteert de diversiteit aan perspectieven in verschillende relevante disciplines. Ons conceptueel raamwerk is gebaseerd op een geïntegreerde benadering van zowel gezondheid als mondialisering en leidt tot waardevolle inzichten in hoe de verschillende relevante factoren op een gestructureerde wijze onderzocht kunnen worden.

Door de complexe interactie tussen de verschillende factoren, zijn er diverse toekomstontwikkelingen in het mondiale system mogelijk. Hoofdstuk 5 verkent onze toekomstige gezondheid, gebruikmakend van meerdere alternatieve mondiale 'toekomstpaden', voor zowel de westerse als nietwesterse wereld. Op basis van negen geselecteerde mondiale scenariostudies (met in totaal 35 scenario's) concluderen we dat de gezondheidsdimensie grotendeels mist in bestaande scenario-exercities. Mondialisering, aan de andere kant, wordt wel in alle scenario's beschreven, meestal als een van de belangrijkste drijvers achter de verschillende verhaallijnen. Onder de geselecteerde scenario's, kan de volgende set van mondiale toekomstpaden worden geïdentificeerd: een mondialiserende en marktgeoriënteerde wereld, een mondialiserende en duurzame wereld en een gefragmenteerde wereld. In elke groep kunnen twee varianten worden onderscheiden. In deze studie worden de institutionele, economische, sociaal-culturele, en ecologische ontwikkelingen beschreven in drie bestaande scenariostudies (SRES, GEO3, MA) gekoppeld aan een set mogelijke toekomstige gezondheidssituaties ('opkomende infectieziekten', 'medische technologie, 'duurzame gezondheid', en 'chronische ziekten in de niet-westerse wereld'). Voor de MA scenario's maken we hierbij gebruik van het eerder ontwikkelde conceptuele raamwerk. 
Deze analyse resulteert in fundamenteel verschillende toekomstontwikkelingen in gezondheid. De studie leidt tot een beter inzicht in de wijze waarop we met gezondheid in scenario's kunnen omgaan en toont aan dat een geïntegreerd beeld van onze toekomstige gezondheid ontstaat wanneer alle relevante institutionele, economische, sociaal-culturele en ecologische ontwikkelingen in acht worden genomen.

De volgende twee hoofdstukken richten zich op de gezondheidseffecten van mondiale milieuverandering. Hoofdstuk 6 onderzoekt de veranderingen in temperatuurgerelateerde sterfte in Nederland ten gevolge van klimaatverandering. Hoewel klimaatverandering naar verwachting gepaard gaat met warmere zomers, zullen winters in alle waarschijnlijkheid ook milder worden. Voor gematigde en koude gebieden (zoals Nederland), verwacht men dan ook dat de toegenomen'hittesterfte' mogelijk wordt gecompenseerd door een afgenomen 'koudesterfte'; dit zou zelfs kunnen resulteren in een afname in de totale temperatuurgerelateerde sterfte. In deze studie wordt de relatie tussen mortaliteit en (extreme) temperatuur bepaald met epidemiologische onderzoeksmethoden (tijdserie analyse en episode analyse). Op basis van de resulterende blootstelling-effect relaties, verkennen we de verandering in de proportie temperatuurgerelateerde sterfte (totale mortaliteit, cardiovasculaire mortaliteit, respiratoire mortaliteit, en mortaliteit in de 65+ leeftijdsgroep) als gevolg van klimaatverandering, gebruikmakend van recente schattingen van dagelijkse temperatuur in 2050 (KNMI'06 scenario's). Onze resultaten tonen aan dat in de twee scenario's zonder verandering in luchtstromingspatronen ( $G$ en $W$ ) de temperatuurgerelateerde sterfte inderdaad afneemt; wanneer we echter in acht nemen dat mensen zich mogelijk aan het nieuwe klimaat aanpassen door middel van acclimatisatie, blijft de temperatuurgerelateerde sterfte in deze scenario's vrijwel onveranderd. De verandering in luchtstromingspatronen in de andere twee scenario's (G+ en $\mathrm{W}+$ ) levert echter andere resultaten op; temperatuurgerelateerde sterfte neemt nog steeds af zonder acclimatisatie, maar stijgt juist met acclimatisatie.

Hoofdstuk 7 onderzoekt met behulp van regressie analyse de kwantitatieve associatie tussen verlies aan biodiversiteit en gezondheid, gebruikmakend van geselecteerde indicatoren voor gezondheid, verlies aan biodiversiteit en sociaaleconomische ontwikkeling op landenniveau. Het mondiale verlies aan biodiversiteit kan resulteren in verstoorde ecosysteem functies en, vervolgens, in negatieve gezondheidseffecten. Er wordt dus een negatieve relatie tussen verlies aan biodiversiteit en gezondheid verondersteld. De resultaten van deze studie kunnen deze negatieve associatie echter niet aantonen. Er kunnen verscheidene redenen voor onze bevindingen zijn: gebrek aan geschikte indicatoren, mogelijke non-randomness in de selectie van landen, beperkingen 
van regressieanalyse, mogelijke non-lineariteit van de onderzochte relatie, de rol van sociaaleconomische ontwikkeling (bijvoorbeeld de scheiding tussen consumptie en consequentie), en het mogelijk ongeschikte schaalniveau. Met andere woorden, de methodologische uitdagingen en de behoefte aan nieuwe innovatieve methoden worden perfect door deze studie geilllustreerd.

Hoofdstuk 8 recapituleert de belangrijkste conclusies van dit proefschrift en plaatst deze in perspectief van de onderzoeksdoelstellingen. Tevens biedt dit hoofdstuk een methodologische reflectie. De centrale vraag in deze studie is hoe onze gezondheid zich in de toekomst ontwikkelt als gevolg van mogelijke mondialiseringstrends. Het antwoord op deze vraag kan echter niet in de voorspelling van één enkel toekomstbeeld worden gevangen. Een belangrijke doelstelling van dit promotieonderzoek is het verkrijgen van een verbeterd inzicht in de mogelijke (toekomstige) gezondheidsimplicaties van mondialisering om zodoende beter te kunnen anticiperen op toekomstige kansen en risico's. De methodologische benadering die aan deze studie ten grondslag ligt kan een bijdrage leveren aan de huidige discussies en ontwikkelingen omtrent het onderzoek naar toekomstige gezondheid in een mondialiserende wereld. Retrospectief wordt er beschreven hoe we de methodologische uitdagingen (holisme en systeemdenken, onzekerheid en pluraliteit, inter- en transdisciplinariteit) binnen ons onderzoek hebben geadresseerd. Een belangrijke toekomstige onderzoekslijn is het verder ontwikkelen van geïntegreerde mondiale scenario's voor gezondheid middels een combinatie van geschikte methoden. Met betrekking tot de gezondheidseffecten van mondiale milieuveranderingen onderstrepen wij het belang van innovatieve systeembenaderingen. Tot slot wijzen wij er kort op dat de internationale beleidsagenda ook moet erkennen dat mondialisering een belangrijke gezondheidsdeterminant is. 


\section{DANKWOORD \& ACKNOWLEDGEMENTS}

This research was financially supported by the Netherlands Environmental Assessment Agency (MNP).

Vaak wordt een promotie traject beschreven als een lang en moeizaam proces, gekenmerkt door vele worstelingen, met het onderzoek en met jezelf. Terugkijkend op de afgelopen jaren, kan ik in alle eerlijkheid zeggen dat ik mijn eigen promotietraject toch met heel andere ogen bekijk. Natuurlijk waren er de bekende struikelblokken en ben ik mijn eigen beperkingen meer dan eens tegengekomen. Maar ik heb naast dit alles toch vooral genoten en ik ben uitermate trots op het eindresultaat.

Ik wil hier dan ook de vele mensen bedanken die door hun steun, advies, commentaar en inspiratie een belangrijke bijdrage hebben geleverd aan mijn promotieonderzoek en werkplezier.

Allereerst wil ik mijn promotor, prof. Pim Martens, bedanken. Pim, jouw deur staat altijd open en dat is voor mij tijdens mijn onderzoek van onschatbare waarde geweest. Altijd kan ik binnenlopen voor een 'vraagje' en regelmatig sta ik dan na een half uur nog steeds op je kamer. Je hebt mij de afgelopen jaren veel geleerd, over wetenschap en over wetenschappers. Tijdens mijn promotietraject heb je me de vrijheid gegeven om mijn eigen weg te vinden binnen het onderzoeksveld 'Global Health'. Dit heb ik zeer gewaardeerd. En toen in de eindfase mijn proefschrift nog steeds niet op je bureau lag, wist je me ook zeker de broodnodige 'schop onder mijn kont' te geven. En met resultaat! $\mathrm{Na}$ het behalen van deze mijlpaal kijk ik dan ook erg uit naar onze verdere samenwerking binnen het International Centre for Integrated Assessment en Sustainable development (ICIS) in de komende periode.

Mijn copromotor, dr. Henk Hilderink, is tijdens mijn promotietraject ook onmisbaar gebleken. Henk, vanuit het Milieu- en Natuurplanbureau (MNP) wierp jij van tijd tot tijd een frisse blik op mijn werk. Daarbij wist je regelmatig op een nauwkeurige, doch vriendelijke, wijze je vinger op de zere plek(ken) van mijn onderzoek te leggen, zodat ik gedwongen werd om nog een keer mijn tanden in de materie te zetten. Onze samenwerking is voor mij erg belangrijk geweest en ik ben je daarvoor veel dank verschuldigd.

De leden van mijn beoordelingscommissie- prof. Wim Passchier, dr. Rene Kemp, prof. Johan Mackenbach en prof. Colin Soskolne- wil ik graag bedanken voor het beoordelen van mijn manuscript. Wim, ik heb het zeer gewaardeerd dat je het voorzitterschap van mijn commissie met zoveel professionaliteit en enthousiasme op je hebt genomen. Rene, bedankt voor je kritische blik en 
commentaar. Johan, bedankt voor de prettige samenwerking in het afgelopen jaar. Ik kijk er naar uit om binnen de 'Initiatiefgroep Mondiale Milieuveranderingen en Volksgezondheid' onze samenwerking in de toekomst voort te zetten. Colin, many, many, many thanks for your thoughtful and thorough comments on this Ph.D. thesis. Additionally, I would like to thank you for all your work in the context of the Global Ecological Integrity Group (GEIG); the past GEIG-meetings have been really inspiring.

Ik ben het MNP zeer erkentelijk voor de financiële ondersteuning van mijn promotieonderzoek. Dr. Guus de Hollander was in het begin van mijn promotietraject mijn voornaamste contactpersoon binnen het MNP. Guus, ik wil je hiervoor graag bedanken. Daarnaast heb ik onze samenwerking in het afgelopen jaar zeer gewaardeerd.

Karlien van den Hout en Laura Vollebregt hebben met hun stage-onderzoek en afstudeerscripties de basis gelegd voor respectievelijk paragraaf 3.2 (de epidemiologische transitie in Nederland) en paragraaf 3.3 (de epidemiologische transitie in Peru) van dit proefschrift. Meiden, bedankt!

Mijn werkplezier heb ik in grote mate te danken aan mijn (ex)collega's bij het ICIS. De afgelopen jaren hebben jullie mij met raad en daad bijgestaan. Binnen de huidige ICIS-groep voel ik me echt als een vis in het water. Dankzij jullie is het halen van een kopje koffie vaak een onverwachte 'social event'. Alvaro, Annet, Annemarie, Anja, Astrid, Bas, Carijn, Darryn, Jan, Machiel, Marc, Mohsin, Pieter, Pim, Rene en Ron: hartelijk dank and thanks a lot!

Van mijn oud-collega's wil ik allereerst Prof. Jan Rotmans en Prof. Marjolein van Asselt bedanken. Jan en Marjolein, jullie boden mij zo'n 10 jaar geleden de kans om als studentassistent kennis te maken met het ICIS. Vanuit de collegebanken belandde ik hierdoor dus direct op de perfecte plek voor mijn promotieonderzoek.

Het voert echter te ver om hier ook alle andere oud-collega's individueel te benoemen en te bedanken. Daarom een collectief en welgemeend: dank jullie wel. Één oud-collega wil ik hier echter toch nog speciaal noemen: Maurice, bedankt voor al die lekkere winegums!

Mijn paranimfen, Janneke Hogervorst en Tim Nawrot, wil ik graag bedanken voor hun vriendschap. Met jullie beiden heb ik tijdens onze studie Milieugezondheidkunde (MGK) aan Universiteit Maastricht een hechte band opgebouwd. Helaas zien we elkaar nu veel minder vaak; onze agenda's zijn na ons afstuderen nu eenmaal een stuk voller geworden. Ik vind het geweldig dat jullie mij tijdens de verdediging van dit proefschrift komen steunen. Janneke, ik 
kan vooral erg genieten van je geweldige gevoel voor humor. In de praktijk komt dit er op neer dat we samen al heel wat hebben afgelachen. Maar ook voor een serieus gesprek kan ik zeker bij jou terecht. Daarnaast ben je (meestal) een perfecte navigator tijdens uitstapjes. Bedankt! Tim, al onze medestudenten waren uiteraard erg milieubewust, maar jij ben toch zeker de enige echte (en ietwat chaotische) 'milieuridder'. Blijf vooral zoals je bent!

Mijn overige studiegenoten wil ik hier ook bedanken. Debby, Rob, Simone en Vivianne: ik kan onze MGK-weekendjes altijd zeer waarderen. Het wordt dus de hoogste tijd om er snel weer eentje te organiseren.

Wie ook zeker niet mogen ontbreken in dit dankwoord zijn de medewerkers van Rugkliniek Heerlen. Milan en Trine, jullie zijn menigmaal mijn redders in nood geweest. Bedankt voor alle goede zorgen.

Bijzonder veel dank ben ik verschuldigd aan mijn familie en vrienden voor hun belangstelling en support.

Mijn ouders, Huub en Thea Huynen, wil ik vanuit het diepst van mijn hart bedanken voor het warme nest waarin ik ben opgegroeid en waarop ik nog altijd kan terugvallen. Mam en pap, ik heb me altijd zeer gesteund gevoeld door jullie rotsvaste vertouwen en liefde.

Simone en Tom, bedankt voor alle steun en 'het luisterende oor'. Simone, je bent echt de allerbeste zus! Emma en Jack, bedankt voor alle lieve knuffels.

Ook mijn schoonfamilie behoort tot de 'harde kern' van mijn supporters. Johan en Mieke, ik voel me altijd ontzettend thuis bij jullie. Bedankt!

Laatst en allerliefst: Merlijn. Het is echt heerlijk om iemand te hebben die mij door en door kent. In de perioden dat ik het ontzettend druk had met mijn onderzoek en het afronden van dit proefschrift, was jij mijn rustpunt, steun en toeverlaat. Jij weet onder alle omstandigheden een lach op mijn gezicht te toveren. Daarnaast wijs je me er op om vooral te genieten van alle goede dingen in het leven. Jij bent mijn allerbeste vriend! 


\section{CURRICULUM VITAE}

Maud Huynen was born in Heerlen on April $4^{\text {th }} 1978$. After completing her secondary education (Bernardinus College, Heerlen), she enrolled as a student Environmental Health Sciences at Maastricht University in 1996. In 1999-2000, she also followed the M.Sc. program in Epidemiology at the same university. She received a M.Sc. Degree in Environmental Health Science with 'Clear Pass' in 2001, and a M.Sc. Degree in Epidemiology in 2003.

In 1998, Maud joined the International Centre for Integrated assessment and Sustainable development (ICIS, Maastricht University) as a student-assistant. After obtaining her master degree, Maud continued working at ICIS on several projects related to the health impacts of (global) environmental change. Her Ph.D. research 'Future health in a globalising world' resulted in this thesis.

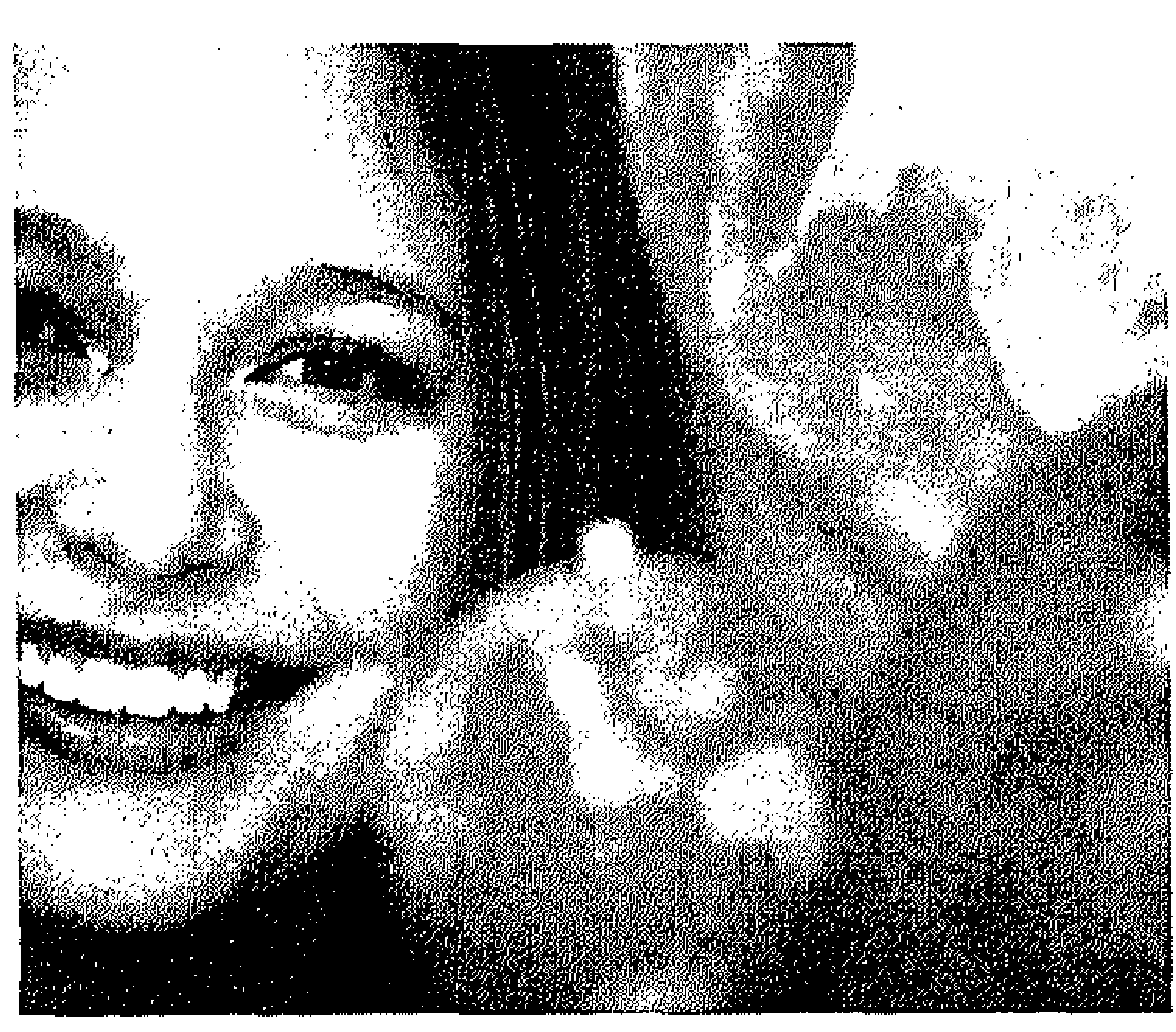

In 2001-2002, Maud was the Assistant Editor of the international journal Global Change and Human Health. At the moment, she is a member of the Editorial Board of the international journal Globalization and Health.

Maud teaches in several courses at Maastricht University on topics related to sustainable development, global health, globalisation, and integrated assessment.

Maud currently works at ICIS as a researcher on topics related to global and environmental health. 